\title{
Classificação de módulos de peso sobre álgebras de Weyl
}

\author{
André Silva de Oliveira \\ DisSERTAÇÃO APRESENTADA \\ $\mathrm{AO}$ \\ Instituto DE MATEMÁtica E EstatísticA \\ $\mathrm{DA}$ \\ Universidade DE SÃo PAUlo \\ PARA \\ OBTENÇÃO DO TÍTULO \\ $\mathrm{DE}$ \\ Mestre em Matemática \\ Programa: Mestrado em Matemática \\ Orientador: Prof. Dr. Vyacheslav Futorny
}

Durante o desenvolvimento deste trabalho o autor recebeu auxílio financeiro da CNPq (Processo 164261/2014-1)

São Paulo, fevereiro de 2016 


\section{Classificação de módulos de peso sobre álgebras de Weyl}

Esta versão da dissertação contém as correções e alterações sugeridas pela Comissão Julgadora durante a defesa da versão original do trabalho, realizada em 28/04/2016. Uma cópia da versão original está disponível no

Instituto de Matemática e Estatística da Universidade de São Paulo.

Comissão Julgadora:

- Prof. Dr. Vyacheslav Futorny - IME-USP

- Prof. Dr. Viktor Bekkert - UFMG

- Prof. Dr. Kostiantyn Iusenko - IME-USP 


\section{Resumo}

OLIVEIRA, A. S. Classificação de módulos de peso sobre álgebras de Weyl. 2016. 161 f. Dissertação (Mestrado) - Instituto de Matemática e Estatística, Universidade de São Paulo, São Paulo, 2016.

Neste trabalho, introduzimos as álgebras de Weyl clássicas $A=A_{n}$ e as generalizadas $A=D(\underline{\sigma}, \underline{a})$. Apresentamos algumas propriedades importantes dessas álgebras, dentre outras, que a $n$-ésima álgebra de Weyl $A_{n}$ é um domínio simples Noetheriano à esquerda. Introduzimos os módulos de peso sobre $A$ e estudamos os $A$-módulos de peso projetivos. Iniciamos a classificação dos $A$-módulos de peso simples (isto é, irredutíveis) através de uma categoria linear $\mathcal{C}_{\mathcal{O}}$ e do seu esqueleto $\mathcal{S}_{\mathcal{O}}$ cf. [BBF04]. A classificação total dos $A_{\infty}$-módulos de peso simples é dada utilizando a ação de certas localizações no anel de polinômios cf. [FGM14]. Classificamos os blocos do tipo mansa na categoria dos $A$-módulos de peso localmente finitos e determinamos os $A$-módulos indecomponíveis nos blocos do tipo mansa. Seguindo [FGM14], descrevemos os $A$-módulos de peso injetivos e projetivos indecomponíveis e deduzimos uma descrição dos blocos na categoria dos $A$-módulos de peso por quivers e relações.

Palavras-chave: álgebras de Weyl, módulos de peso simples, módulos de peso indecomponíveis. 


\section{Abstract}

OLIVEIRA, A. S. Classification of weight modules over Weyl algebras. 2016. $161 \mathrm{f}$. Dissertation (Masters) - Instituto de Matemática e Estatística, Universidade de São Paulo, São Paulo, 2016.

In this dissertation, we introduce the classical Weyl algebras $A=A_{n}$ and the generalized $A=D(\underline{\sigma}, \underline{a})$. There are some important properties of these algebras, among others, that the $n$-th Weyl algebra $A_{n}$ is a left Noetherian simple domain. We introduced the weight modules over $A$ and study the projective weight $A$-modules. Started the classification of simple weight $A$-modules (this is, irreducible) by linear category $\mathcal{C}_{\mathcal{O}}$ and its skeleton $\mathcal{S}_{\mathcal{O}}$ in accordance with [BBF04]. The complete classification of simple weight $A$-modules is given using the action of certain localizations in the polynomial ring in accordance with [FGM14]. We classify the tame blocks in the category of locally-finite weight $A$-modules and determine the indecomposable $A$-modules in the tame blocks. Following [FGM14], we describe indecomposable projective and injective weight $A$-modules and deduce the description of the blocks in the category of weight $A$-modules by quivers and relations.

Keywords: Weyl algebras, simple weight modules, indecomposable weight modules. 


\section{Sumário}

Introdução $\quad$ ix

0.1 Considerações Preliminares . . . . . . . . . . . . . . ix

0.2 Organização do Trabalho . . . . . . . . . . . . . . . . . ix

1 Álgebras de Weyl $\quad 1$

1.1 Conceitos Básicos . . . . . . . . . . . . . . . . . 2

1.1 .1 Forma canônica . . . . . . . . . . . . . . . . 5

1.1.2 Geradores e relações . . . . . . . . . . . . . . . . 8

1.1.3 O grau de um operador em $A_{n} \ldots \ldots \ldots \ldots$

1.1.4 Anel de operadores diferenciais . . . . . . . . . . . . . 12

1.2 Problemas com corpos com característica prima . . . . . . . . . . . 17

1.3 Álgebra de Weyl de posto infinito . . . . . . . . . . . . . . . . . . 17

1.4 Álgebra de Weyl generalizada . . . . . . . . . . . . . . . . . 25

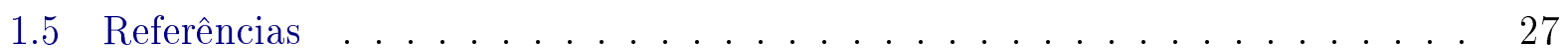

2 Módulos sobre a álgebra de Weyl 29

2.1 Módulos sobre a álgebra de Weyl $A_{n} \ldots \ldots \ldots \ldots$

2.2 Módulos graduados e filtrados . . . . . . . . . . . . . . . . . 32

2.2.1 Anéis e módulos graduados . . . . . . . . . . . . . . 32

2.2 .2 Anéis filtrados . . . . . . . . . . . . . . . 34

2.2 .3 Álgebra graduada associada ..................... 35

2.2.4 Módulos filtrados . . . . . . . . . . . . . . . . . 37

2.2.5 Filtrações induzidas . . . . . . . . . . . . . . . 39

2.3 Anéis e módulos Noetherianos . . . . . . . . . . . . . . . . . . 41

2.3.1 Anéis Noetherianos . . . . . . . . . . . . . . . . . . 42

2.4 Módulos de peso sobre $A=A_{k, \mathrm{I}} \ldots \ldots \ldots \ldots \ldots$. . . . . . . 44

2.5 Módulos de peso projetivos sobre $A=A_{k, \mathrm{I}} \ldots \ldots \ldots \ldots$

2.6 Involução e dualidade restrita . . . . . . . . . . . . . . . 57

2.7 Realização via ação polinomial . . . . . . . . . . . . . . . . . . 62

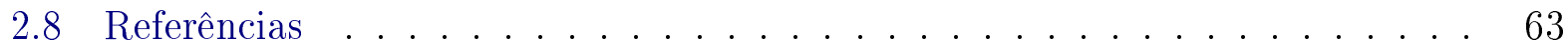


3 Classificação

3.1 Módulos de peso simples sobre uma álgebra de Weyl generalizada $\mathrm{A}=\mathrm{D}(\underline{\sigma}, \underline{\mathrm{a}}) 65$

3.1.1 Módulos de peso sobre $\mathrm{A}=\mathrm{D}(\underline{\sigma}, \underline{\mathrm{a}}) \ldots \ldots \ldots . \ldots . \ldots 6$

3.1 .2 Categoria $\mathcal{C}_{\mathcal{O}}$ e seu esqueleto . . . . . . . . . . . . 67

3.1 .3 Descrição dos módulos de peso simples . . . . . . . . . . . . . 73

3.1 .4 Exemplo: caso $\mathrm{A}_{1} \ldots \ldots \ldots \ldots \ldots$. . . . . . . . . . 77

3.2 Tipo de representação e módulos indecomponíveis para $A_{n}, 1 \leq n \leq \infty$. . 77

$3.2 .1 \quad$ Indecomponíveis para $\mathcal{A}(\mathbb{F}, I),|I|=1 \ldots \ldots \ldots \ldots$

3.2 .2 Indecomponíveis para $\mathcal{A}(\mathbb{F}, I),|I|=2 \ldots \ldots \ldots \ldots$

3.2 .3 Indecomponíveis para blocos do tipo mansa . . . . . . . . . . 80

3.3 Módulos de peso simples sobre $A=A_{k, \mathrm{I}} \ldots \ldots \ldots \ldots$

3.4 Realizações via polinômios torcidos . . . . . . . . . . . . . . . . 84

3.5 Localização das realizações . . . . . . . . . . . . . . . . . 87

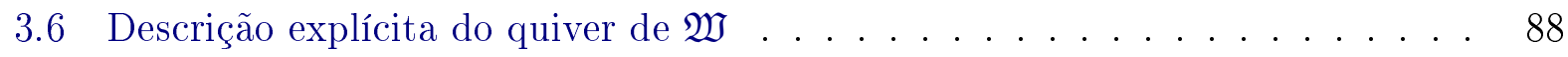

3.7 Koszulidade . . . . . . . . . . . . . . . . . . . 89

3.8 Descrição dos blocos . . . . . . . . . . . . . . . . . . 91

3.9 Referências . . . . . . . . . . . . . . . . . 91

$\begin{array}{ll}\text { A Âlgebras de Lie } & 93\end{array}$

A.1 Conceitos Básicos . . . . . . . . . . . . . . . . . . 93

A.2 Álgebras de Lie . . . . . . . . . . . . . . . . . . . 97

A.2.1 Generalidades . . . . . . . . . . . . . . . . . 97

A.2.2 Derivações de álgebras de Lie . . . . . . . . . . . . . . . 99

A.2.3 Ideais e homomorfismos . . . . . . . . . . . . . . 99

A.2.4 Álgebras de Lie Solúveis e Nilpotentes . . . . . . . . . . . . . 101

A.2.5 Teoremas de Lie e Cartan . . . . . . . . . . . . . . 103

A.2.6 Representações de $\mathfrak{s l}(2, k) \quad \ldots \ldots \ldots$. . . . . . . . . 105

A.3 Teoria de estrutura . . . . . . . . . . . . . . . . 106

A.3.1 Decomposição de Cartan . . . . . . . . . . . . . . . 106

A.3.2 Sistema de raízes . . . . . . . . . . . . . . . . . . 108

A.3.3 Subálgebra de Cartan . . . . . . . . . . . . . . . . 109

A.4 Álgebras Envolventes Universais . . . . . . . . . . . . . . . . . 109

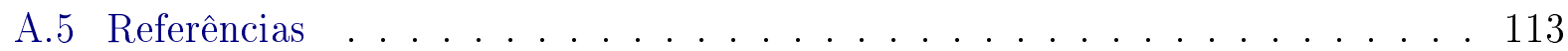

B Teoria de Categorias $\quad 115$

B.1 Categorias e Funtores . . . . . . . . . . . . . . . . . 115

B.2 Categorias Aditivas e Abelianas . . . . . . . . . . . . . . . 122

B.3 Categorias R-mod e mod-R . . . . . . . . . . . . . 125

B.3.1 Funtores covariantes de R-mod em S-mod . . . . . . . . . . . . 125

B.3.2 Funtores contravariantes de R-mod em S-mod . . . . . . . . . . . 127 
B.3.3 Bifuntores . . . . . . . . . . . . . . . 128

B.3.4 O funtor Hom . . . . . . . . . . . . . . . . 129

B.3.5 Módulos Projetivos . . . . . . . . . . . . 130

B.3.6 Módulos Injetivos . . . . . . . . . . . . . . . . . 132

B.3.7 O funtor Tensor . . . . . . . . . . . . . . . . . 133

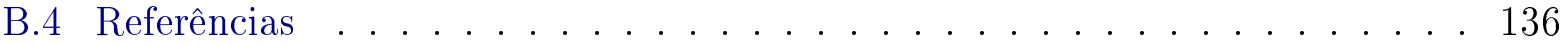

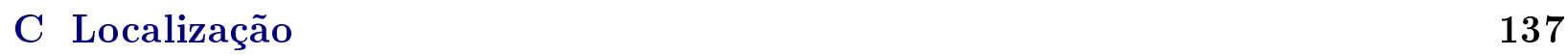

C.1 Localizações em anéis comutativos . . . . . . . . . . . . . . . 137

C.2 Localizações em anéis não comutativos . . . . . . . . . . . . . . 138

C.3 Localizações em módulos . . . . . . . . . . . . . . . . . . . . 142

C.4 Referências . . . . . . . . . . . . . . . . . . . 143

Referências Bibliográficas 145 


\section{Introdução}

\subsection{Considerações Preliminares}

As álgebras de Weyl são objetos de estudo clássicos em teoria de representações de álgebras associativas, que surgem naturalmente em matemática e em física, e tem muitas importantes aplicações. Por exemplo, uma parte essencial da abordagem dos $\mathcal{D}$-módulos, em que $\mathcal{D}$ é um anel de operadores diferenciais, para a teoria de representação de uma álgebra de Lie $\mathfrak{g}$ simples de dimensão finita é a existência de um homomorfismo natural de $U(\mathfrak{g})$ (álgebra envolvente universal de $\mathfrak{g}$ ) para uma álgebra de Weyl $A_{n}$ de posto finito. No caso de uma álgebra de Lie afim existe um homomorfismo similar, mas agora para uma álgebra de Weyl $A_{\infty}$ de posto infinito.

A álgebra $A_{\infty}$ pode ser vista como uma álgebra de Weyl generalizada de posto infinito e então temos uma categoria natural de representações consistindo dos então chamados módulos de peso. Tais módulos sobre álgebras de Weyl de posto finito ou infinito tem sido extensivamente estudados nos últimos 20 anos. Várias construções e resultados de classificações aparecem em [BB00], [BBF04] e [GS06]. Em particular, uma classificação parcial de $A_{\infty}$-módulos de peso simples foi dada em [BBF04] e uma total em [FGM14].

\subsection{Organização do Trabalho}

No Capítulo 1 apresentamos as álgebras de Weyl. Na Seção 1.1 introduzimos a $n$-ésima álgebra de Weyl $A_{n}(k)$ com $2 n$ geradores, sobre um corpo $k$ de característica 0 , de duas maneiras diferentes: como um quociente de uma $k$-álgebra livre por um ideal de relações e como um anel de operadores diferenciais sobre o anel dos polinômios em $n$ variáveis comutativas $k\left[x_{1}, \ldots, x_{n}\right]$, ou seja, mostramos que

$$
D\left(k\left[x_{1}, \ldots, x_{n}\right]\right)=A_{n}(k) .
$$

Ainda na Seção 1.1, apresentamos algumas propriedades dessa álgebra, tais como, $A_{n}(k)$ é um domínio e um anel simples. Na Seção 1.2 discutimos alguns problemas que podem ocorrer caso o corpo base $k$ tenha característica prima.

Na Seção 1.3 exigimos que o corpo base $k$, além de ter característica 0 , fosse algebricamente fechado. Tomamos um conjunto I, infinito e enumerável, satisfazendo a seguinte 
condição: $|\mathrm{I}|<|k|$, e definimos uma $k$-álgebra $B$ de polinômios com uma quantidade infinita de variáveis comutativas $x_{i}, i \in \mathrm{I}$. Partindo dessa $k$-álgebra, definimos a $k$-álgebra de Weyl de posto infinito $A=A_{k, \mathrm{I}}$ com geradores $X_{i}, Y_{i}, i \in \mathrm{I}$, em que $X_{i}$ é o operador linear de $B$ que age por multiplicação pela variável $x_{i}$, enquanto $Y_{i}$ é o operador que age derivando

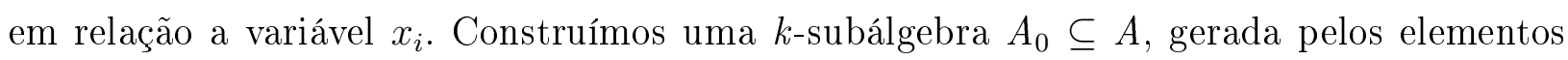
$t_{i}=X_{i} Y_{i}=x_{i} \partial_{i}, i \in \mathrm{I}$. Provamos que $A_{0}$ é $k$-subálgebra comutativa maximal em $A$ e que o conjunto de todos os $t_{i}$ 's são algebricamente independentes sobre $k$, logo $A_{0} \cong k\left[t_{i} \mid i \in \mathrm{I}\right]$. Além disso, descrevemos os ideais maximais em $A_{0}$. Finalizamos o capítulo com a Seção 1.4, em que apresentamos as álgebras de Weyl generalizadas que foram introduzidas por Bavula [Bav92a], e que desempenham um papel muito importante na classificação dos módulos sobre as álgebras de Weyl.

No Capítulo 2, estudamos os módulos sobre as álgebras de Weyl. Na Seção 2.1 consideramos módulos sobre a $n$-ésima álgebra de Weyl $A_{n}(k)$ e mostramos que $k\left[x_{1}, \ldots, x_{n}\right]$ é um $A_{n}(k)$-módulo irredutível de torção e além disso

$$
k\left[x_{1}, \ldots, x_{n}\right] \cong A_{n}(k) / \sum_{i=1}^{n} A_{n}(k) \cdot \partial_{i} .
$$

Na Seção 2.2 mostramos duas importantes filtrações da álgebra $A_{n}(k)$, no caso a filtração de Bernstein $\mathcal{B}=B_{r}\left(A_{n}(k)\right)$ e a filtração ordem $\mathcal{C}$. Utilizando a filtração de Bernstein mostramos que $S_{n}=g r^{\mathcal{B}} A_{n}(k)$, a álgebra graduada de $A_{n}(k)$ associada com a filtração $\mathcal{B}$, é isomorfa ao anel de polinômios sobre $k$ com $2 n$ variáveis. Na Seção 2.3 provamos o Teorema da Base de Hilbert e mostramos que $A_{n}(k)$ é um anel Noetheriano à esquerda.

Na Seção 2.4 voltamos nossa atenção para a álgebra de Weyl $A=A_{k, \mathrm{I}}$ de posto infinito e para os $A$-módulos (cf. [FGM14]). Definimos um $A$-módulo de peso e consideramos $\mathfrak{W}$ como a subcategoria plena de $A$-mod consistindo de todos os $A$-módulos de peso. Para $\mathbf{p} \in k^{\mathrm{I}}$, denotamos por $\mathfrak{W}_{\mathbf{p}}$ a subcategoria plena de $\mathfrak{W}$ consistindo de todos os $A$-módulos de peso $M$ tais que $\operatorname{supp}(M) \subseteq \mathbf{p}+\mathbb{Z}_{f}^{\mathrm{I}}$ e explicitamos a seguinte decomposição usual

$$
\mathfrak{W} \cong \bigoplus_{\xi \in k^{\mathrm{I}} / \mathbb{Z}_{f}^{\mathrm{I}}} \mathfrak{W}_{\mathbf{p}_{\xi}}
$$

em que $\mathbf{p}_{\xi} \in \xi$ é algum representante fixo da classe $\xi$ em $k^{\mathrm{I}} / \mathbb{Z}_{f}^{\mathrm{I}}$.

Na Seção 2.5, consideramos para $\mathbf{p} \in k^{\mathrm{I}}$, a subcategoria plena $\mathfrak{V}_{\mathbf{p}}$ de $A_{0}$ - $\bmod$ consistindo de todos $A_{0}$-módulos $N$ tais que $\mathfrak{m}_{\mathbf{p}} \cdot N=\{0\}$. Definimos um par de funtores adjuntos exatos:

$$
R e s_{\mathbf{p}}: \mathfrak{W} \rightarrow \mathfrak{V}_{\mathbf{p}} \quad \text { e } \quad \operatorname{Ind}_{\mathbf{p}}=: A \otimes_{A_{0}}-: \mathfrak{V}_{\mathbf{p}} \rightarrow \mathfrak{W}
$$

funtor restrição e indução, respectivamente, e através disso discutimos algumas propriedades de $P(\mathbf{p})$ e $L(\mathbf{p})$, um $A$-módulo de peso projetivo e seu topo, respectivamente. Encerramos a seção provando que $P(\mathbf{p})$ é a cobertura projetiva de $L(\mathbf{p})$. 
Na Seção 2.6 consideramos $\mathfrak{W}^{f}$, a subcategoria plena de $\mathfrak{W}$ consistindo de todos os $A$ módulos de peso com espaços de peso de dimensão finita. Temos que $P(\mathbf{p})$ e $L(\mathbf{p}) \in \mathfrak{W}^{f}$. Definimos uma dualidade restrita canônica $\vee$ nesta subcategoria. Para $M \in \mathfrak{W}^{f}$ temos que:

$$
M^{\vee}:=\bigoplus_{\mathbf{p} \in k^{\mathrm{I}}} \operatorname{Hom}_{k}\left(M_{\mathbf{p}}, k\right)
$$

e como $L(\mathbf{p})^{\vee} \cong L(\mathbf{p})$, temos que $P(\mathbf{p})^{\vee}$ é o envelope injetivo de $L(\mathbf{p})$. Finalizamos o capítulo com a Seção 2.7, em que apresentamos uma realização do $A$-módulo de peso projetivo $P(\mathbf{p})$.

No Capítulo 3, na Seção 3.1, descrevemos os módulos de peso sobre a álgebra de Weyl generalizada $A=D(\underline{\sigma}, \underline{a})$ e estudamos a ação de um grupo $\mathcal{G}$ (gerado pelos automorfismos $\left.\sigma_{i}\left(\tilde{t_{j}}\right)=\tilde{t_{j}}-\delta_{i j} 1\right)$ que age no conjunto dos ideais maximais de $D, \mathfrak{m a x} D$. Definimos uma categoria linear $\mathcal{C}_{\mathcal{O}}$ (uma categoria definida a partir de uma órbita $\mathcal{O}$, que foi primeiramente introduzida em [DGO96]) e provamos que $\mathcal{W}_{\mathcal{O}}(A)$ (a categoria dos $A$-módulos de peso com órbita $\mathcal{O}$ ) é equivalente a categoria $\mathcal{C}_{\mathcal{O}}$ - $\bmod$ (categoria dos módulos sobre $\mathcal{C}_{\mathcal{O}}$ ). Exibimos também um esqueleto $\mathcal{S}_{\mathcal{O}}$ de $\mathcal{C}_{\mathcal{O}}$ e o descrevemos algebricamente. Descrevemos também os módulos simples sobre $A$, em que esta descrição foi feita via redução à dois tipos de categorias lineares chamadas de $\mathcal{A}(\mathbb{F}, I)$ e $\mathcal{B}(\mathbb{F}, I, J, \underline{\tau})$. Encerrando a seção, classificamos os $A$-módulos de peso simples, para um corpo $k$ qualquer.

Na Seção 3.2 classificamos os blocos do tipo mansa na categoria $\mathcal{W}_{\mathcal{O}}^{l f}(A)$ (dos módulos de peso localmente finitos sobre $A$ com órbita $\mathcal{O})$. Segue de [BB00][Seção 2.6] que $\mathcal{B}(\mathbb{F}, I, J, \tau)$ é selvagem se $|I|+|J|>1$, e $\mathcal{A}(\mathbb{F}, I)$ é selvagem para $|I|>2$. Portanto, como o nosso objetivo era descrever os indecomponíveis nos blocos do tipo mansa, foi suficiente descrever os indecomponíveis para as categorias $\mathcal{A}(\mathbb{F}, I) \operatorname{com}|I| \leq 2$ e para $\mathcal{B}(\mathbb{F}, I, J, \underline{\tau}) \operatorname{com}|I|+|J|=1$ (que corresponde ao caso $n=1$ ).

Na Seção 3.3 (cf. [FGM14]) classificamos os módulos de peso simples sobre $A=A_{k, \mathrm{I}}$ (sem utilizar o conceito de órbita), em que para $\mathbf{p} \in k^{\mathrm{I}}$, a aplicação $\overline{\mathbf{p}} \mapsto L(\mathbf{p})$, é uma bijeção entre $k^{\mathrm{I}} / \sim$ e o conjunto das classes de isomorfismos dos $A$-módulos de peso simples. Inclusive é dado um exemplo de um $A_{\infty}$-módulo de peso simples que não possui break maximal, justificando assim a não utilização do conceito de órbitas para classificar módulos de peso sobre $A_{\infty}$. Na Seção 3.4, realizamos os $A$-módulos de peso simples via polinômios torcidos, utilizando automorfismos $\theta_{\mathrm{J}}$ de $A$. Concluímos que $B(\mathbf{p})^{\theta_{\mathrm{J}}}$ e $\left(B(\mathbf{p})^{\theta_{\mathrm{J}}}\right)^{\vee}$ são o envelope injetivo e a cobertura projetiva de $L(\mathbf{p})^{\theta_{\mathrm{J}}}$, respectivamente. Na Seção 3.5, localizamos essas realizações, tal que $\mathbf{D}_{\mathrm{J}} A$ é a localização de Ore de $A$ com respeito ao subconjunto multiplicativo de Ore gerado por todo $X_{i}$, com $i \in \mathrm{I}$ (visto que a ação adjunta de $X_{i}$ em $A$ é localmente nilpotente). Definimos o funtor $F_{\mathrm{J}}:=\operatorname{Res}_{A}^{\mathbf{D}_{\mathrm{J}} A} \circ \operatorname{Ind}_{A}^{\mathbf{D}_{\mathrm{J}} A}$ na categoria $A$-mod, e provamos que $L(\mathbf{p}) \cong F_{\mathrm{I} \backslash \mathrm{J}_{\mathbf{p}}}^{\mathbf{p}^{\prime}} L(\mathbf{0})$ e $B(\mathbf{p}) \cong F_{\mathrm{J}_{\mathbf{p}}} L(\mathbf{p})$.

Na Seção 3.6, descrevemos um quiver $Q=Q_{E}$ partindo de um conjunto não vazio $E$ e denotamos a respectiva categoria dos caminhos de $Q$ por $C_{E}$. Na Seção 3.7 provamos que a categoria $C_{E}$ é Koszul. Finalizamos o capítulo mostrando que os blocos de $\mathfrak{W}$ (a subcategoria 
plena de $A$-mod consistindo de todos os $A$-módulos de peso, cf. Capítulo 2, Seção 2.4) são descritos por $C_{E}$, para um $E$ apropriado, ou seja, para $\mathrm{J}_{\mathbf{p}} \neq \emptyset$, a categoria $\mathfrak{W}_{\mathbf{p}}$ é equivalente a categoria dos módulos sobre $C_{\mathrm{J}_{\mathbf{p}}}$. 


\section{Capítulo 1}

\section{Álgebras de Weyl}

A história da álgebra de Weyl começa com o nascimento da mecânica quântica. Mecânica quântica é a teoria física que obtém sucesso no estudo dos sistemas físicos cujas dimensões são próximas ou abaixo da escala atômica, tais como moléculas, átomos, elétrons, prótons e outras partículas subatômicas. Em física, chama-se "sistema" um fragmento concreto da realidade que foi separado para estudo. Cada sistema ocupa um estado num instante de tempo. As leis da física devem dizer como o sistema evolui (de estado em estado). Muitos fenômenos quânticos difíceis de se imaginar concretamente podem ser compreendidos com um pouco de abstração matemática. Na mecânica quântica, todos os estados são representados por vetores em um espaço vetorial complexo: o espaço de Hilbert $H$ (na matemática, um espaço de Hilbert é uma generalização do espaço euclideano, ou seja, é um espaço vetorial dotado de um produto interno, com noções de distância e ângulo). Assim cada vetor no espaço $H$ representa um estado que pode ser ocupado pelo sistema.

Os alicerces da mecânica quântica foram estabelecidos durante a primeira metade do século XX por Albert Einstein, Werner Heisenberg, Max Planck, Niels Bohr, Paul Dirac, entre outros. Para Werner Heisenberg essa mecânica tinha que ser baseada nas quantidades que podiam de fato serem observadas, os observáveis. Nas palavras de Paul Dirac: "As coisas que são observadas, ou que estejam ligadas às quantidades observadas, estão todas associadas com duas órbitas com base no modelo atômico de Bohr e não com apenas uma. Suponha que nós consideremos todas as quantidades de um determinado tipo associado com duas órbitas, e queremos descrevê-las. A maneira mais natural de escrever um conjunto de quantidades, cada uma associada à dois elementos é da seguinte forma:

$$
\left(\begin{array}{cccc}
\times & \times & \times & \ldots \\
\times & \times & \times & \ldots \\
\times & \times & \times & \ldots \\
\cdot & \cdot & \cdot & \ldots
\end{array}\right)
$$

uma lista de quantidades que se configura em termos de linhas e colunas. As linhas representam um dos estados e as colunas o outro. Os matemáticos chamam um conjunto de quantidades como este de matriz." O efeito disso foi a introdução das quantidades não comutativas. Assim, juntamente com Max Born e Pascual Jordan, Heisenberg estabeleceu as bases da formulação matricial da mecânica quântica, a "Mecânica Matricial".

No final da década de 1920, Heisenberg formulou o princípio da incerteza. De acordo com esse princípio, não podemos determinar com precisão e simultaneamente a posição e o momento de uma partícula. Pode-se exprimir o princípio da incerteza nos seguintes termos: o produto da incerteza associada ao valor de uma coordenada $x_{i}$ pela incerteza associada ao seu correspondente momento linear $p_{i}$ (o momento linear, uma grandeza vetorial, em 
mecânica clássica é o produto da massa pela velocidade de um corpo, isto é, $\vec{p}=m \cdot \vec{v}$ ) não pode ser inferior, em grandeza, à constante de Planck normalizada. Em termos matemáticos, exprime-se assim:

$$
\Delta x_{i} \Delta p_{i} \geq \frac{\hbar}{2}
$$

em que $\hbar$ é a constante de Planck $(h)$ dividida por $2 \pi$. As álgebras de Weyl foram introduzidas por Hermann Weyl para estudar o princípio de incerteza de Heisenberg em mecânica quântica.

As álgebras de Weyl podem ser definidas de várias maneiras diferentes, mas todas as definições são equivalentes. O leitor pode encontrar mais detalhes em [Cou95].

\subsection{Conceitos Básicos}

Vamos introduzir a álgebra de Weyl como um anel de operadores de um espaço vetorial de dimensão infinita. Vamos fixar algumas notações. Ao longo deste capítulo, $k$ será um corpo de característica zero (o caso em que a característica é prima será tratado na Seção $1.2)$ e $k[X]$ o anel dos polinômios $k\left[x_{1}, \ldots, x_{n}\right]$ em $n$ variáveis comutativas sobre $k$.

$\mathrm{O}$ anel $k[X]$ é um espaço vetorial de dimensão infinita sobre $k$. A $k$-álgebra dos operadores lineares do espaço vetorial $k[X]$ é denotada por $\operatorname{End}_{k}(k[X])$. Observamos que as operações nesta álgebra $\operatorname{End}_{k}(k[X])$ são a adição e composição dos operadores. Então $E n d_{k}(k[X])$ é um anel não comutativo com unidade. A álgebra de Weyl pode ser definida como uma subálgebra de $\operatorname{End}_{k}(k[X])$.

Definição 1.1.1. Sejam $\hat{x}_{1}, \ldots, \hat{x}_{n}$ os operadores lineares de $k[X]$ que são definidos em um polinômio $f \in k[X]$ pela fórmula $\hat{x}_{i}(f)=x_{i} \cdot f, 1 \leq i \leq n$. Similarmente, sejam $\partial_{1}, \ldots, \partial_{n}$ os operadores lineares definidos por $\partial_{i}(f)=\partial f / \partial x_{i}, 1 \leq i \leq n$. A n-ésima álgebra de Weyl $A_{n}$ é a $k$-subálgebra de End $\boldsymbol{E}_{k}(k[X])$ de posto finito gerada pelos operadores $\hat{x}_{1}, \ldots, \hat{x}_{n}$ e $\partial_{1}, \ldots, \partial_{n}$.

Dizemos que $A_{n}$ tem posto finito, pois tem uma quantidade finita de geradores.

Observamos que $\hat{x}_{i}, \partial_{i}, 1 \leq i \leq n$, também podem ser chamados de $k$-endomorfismos de $k[X]$.

Para $m \leq n$, a ação dos operadores de $A_{m}$ em $k[X]$ está bem definida. Então $A_{m}$ é uma subálgebra de $A_{n}$ de modo natural. Podemos escrever $A_{n}(k)$ ao invés de $A_{n}$, se for necessário explicitar o corpo sobre o qual a álgebra está definida.

Temos que $A_{n}$ é uma $k$-álgebra associativa unital. De acordo com a definição, os elementos de $A_{n}$ são combinações lineares sobre $k$ de monômios nos geradores $\hat{x}_{1}, \ldots, \hat{x}_{n}, \partial_{1}, \ldots, \partial_{n}$. Contudo, temos que ser cuidadosos quando representar os elementos de $A_{n}$ porque esta álgebra não é comutativa. Podemos verificar isso facilmente. Considere o operador $\partial_{i} \circ \hat{x}_{i}$ e o aplique em um polinômio $f \in k[X]$, ou seja, $\left(\partial_{i} \circ \hat{x}_{i}\right)(f)=\partial_{i}\left(x_{i} \cdot f\right)$. Utilizando a regra de derivação do produto, temos que $\partial_{i}\left(x_{i} \cdot f\right)=\left(\partial_{i}\left(x_{i}\right)\right) \cdot f+x_{i} \cdot\left(\partial_{i}(f)\right)$. Então $\left(\partial_{i} \circ \hat{x}_{i}\right)(f)=(1)(f)+\left(\hat{x}_{i} \circ \partial_{i}\right)(f)$. Portanto, como $f$ é arbitrário, $\partial_{i} \circ \hat{x}_{i}=1+\hat{x}_{i} \circ \partial_{i}$, em que 1 é o operador identidade. Podemos escrever esta relação usando comutadores. Em uma álgebra associativa, o comutador $[a, b]$ entre dois elementos é dado por $a b-b a$. Então, se $P, Q \in A_{n}$, o comutador entre eles é o operador $[P, Q]=P \circ Q-Q \circ P$. Portanto $\left[\partial_{i}, \hat{x}_{i}\right]=1$.

Agora vamos considerar $\left[\partial_{i}, \hat{x}_{j}\right]$, com $i \neq j$, $\left[\partial_{i}, \partial_{j}\right]$, com $1 \leq i, j \leq n$, e $\left[\hat{x}_{i}, \hat{x}_{j}\right]$, com $1 \leq i, j \leq n$. Seja $f \in k[X]$, um polinômio arbitrário:

- $\left(\partial_{i} \circ \hat{x}_{j}\right)(f)=\partial_{i}\left(x_{j} \cdot f\right)=\left(\partial_{i}\left(x_{j}\right)\right) \cdot f+x_{j} \cdot\left(\partial_{i}(f)\right)=(0)(f)+\left(\hat{x}_{j} \circ \partial_{i}\right)(f)$. Portanto $\partial_{i} \circ \hat{x}_{j}=\hat{x}_{j} \circ \partial_{i}$, ou seja, $\left[\partial_{i}, \hat{x}_{j}\right]=0$, quando $i \neq j$. 
- Sabemos que vale a seguinte igualdade: $\frac{\partial^{2} f}{\partial x_{i} \partial x_{j}}=\frac{\partial^{2} f}{\partial x_{j} \partial x_{i}}$. Então $\left(\partial_{i} \circ \partial_{j}\right)(f)=\left(\partial_{j} \circ\right.$ $\left.\partial_{i}\right)(f)$. Portanto $\left[\partial_{i}, \partial_{j}\right]=0,1 \leq i, j \leq n$.

- Sabemos que as variáveis $x_{i}$ e $x_{j}$ comutam para todo $1 \leq i, j \leq n$. Assim $x_{i} \cdot\left(x_{j} \cdot f\right)=$ $x_{j} \cdot\left(x_{i} \cdot f\right)$. Então $\left(\hat{x}_{i} \circ \hat{x}_{j}\right)(f)=\left(\hat{x}_{j} \circ \hat{x}_{i}\right)(f)$. Portanto $\left[\hat{x}_{i}, \hat{x}_{j}\right]=0,1 \leq i, j \leq n$.

As relações entre os geradores da $n$-ésima álgebra de Weyl $A_{n}$ são resumidas abaixo:

$$
\begin{gathered}
{\left[\partial_{i}, \hat{x}_{j}\right]=\delta_{i j}} \\
{\left[\partial_{i}, \partial_{j}\right]=\left[\hat{x}_{i}, \hat{x}_{j}\right]=0}
\end{gathered}
$$

em que $1 \leq i, j \leq n$ e $\delta_{i j}$ é o símbolo do delta de Kronecker (é igual à 1 se $i=j$ e 0 caso contrário).

Denotamos o operador "multiplicação por $x_{i}$ " pelo símbolo $\hat{x}_{i}$. Escreveremos $x_{i}$ tanto para a variável como para o operador correspondente. Isto torna a notação menos carregada. Pela mesma razão nós vamos dispensar os índices para os geradores de $A_{1}$, e escreveremos simplesmente $x$ e $\partial$. Vamos também dispensar o sinal o para a multiplicação em $A_{n}$.

Proposição 1.1.1. Para $1 \leq i \leq n$, valem as seguintes propriedades:

1. $\left[\partial_{i}, f\right]=\partial_{i}(f)=\partial f / \partial x_{i}$, para cada $f \in k[X]$.

2. $\left[\partial_{i}, x_{i}^{u}\right]=u x_{i}^{u-1}$, para todo $u \in \mathbb{N}^{*}$.

3. $\left[\partial_{i}^{u}, x_{i}\right]=u \partial_{i}^{u-1}$, para todo $u \in \mathbb{N}^{*}$.

4. (Fórmula de Leibniz) Para cada $k, l \in \mathbb{N}$ :

$$
\partial_{i}^{k} x_{i}^{l}=\sum_{j=0}^{k}\left(\begin{array}{l}
k \\
j
\end{array}\right) \partial_{i}^{j}\left(x_{i}^{l}\right) \partial_{i}^{k-j}
$$

em que $\left(\begin{array}{l}k \\ j\end{array}\right)=\frac{k !}{j !(k-j) !}$. Em particular:

$$
\left[\partial_{i}^{k}, x_{i}^{l}\right]=\sum_{j=1}^{k}\left(\begin{array}{l}
k \\
j
\end{array}\right) \partial_{i}^{j}\left(x_{i}^{l}\right) \partial_{i}^{k-j}
$$

Demonstração. 1. Sejam $g \in k[X]$, um polinômio arbitrário, e $f$ o $k$-endomorfismo de $k[X]$ tal que $f(g)=f \cdot g$. Temos que:

$$
\left(\partial_{i} f\right)(g)=\partial_{i}(f \cdot g)=f \cdot\left(\partial_{i}(g)\right)+\left(\partial_{i}(f)\right) \cdot g=\left(f \partial_{i}\right)(g)+\left(\partial f / \partial x_{i}\right)(g)
$$

Portanto $\partial_{i} f-f \partial_{i}=\partial f / \partial x_{i}$, ou melhor, $\left[\partial_{i}, f\right]=\partial f / \partial x_{i}=\partial_{i}(f)$.

2. Podemos considerar $f=x_{i}^{u}$, com $u \in \mathbb{N}^{*}$. Então pelo item 1 , temos que $\left[\partial_{i}, x_{i}^{u}\right]=$ $\partial_{i}\left(x_{i}^{u}\right)=u x_{i}^{u-1}$.

3. Seja $u \in \mathbb{N}^{*}$. Vamos usar indução sobre $u$. Se $u=1$, sabemos que $\left[\partial_{i}, x_{i}\right]=1$, logo o resultado é válido. Suponhamos então que $u>1$ e que o resultado seja válido para $u-1$. Temos que:

$$
\left[\partial_{i}^{u}, x_{i}\right]=\partial_{i}^{u} x_{i}-x_{i} \partial_{i}^{u}=\partial_{i} \partial_{i}^{u-1} x_{i}-x_{i} \partial_{i} \partial_{i}^{u-1}=\partial_{i} \partial_{i}^{u-1} x_{i}-x_{i} \partial_{i} \partial_{i}^{u-1}+\partial_{i} x_{i} \partial_{i}^{u-1}-\partial_{i} x_{i} \partial_{i}^{u-1}
$$


Assim:

$$
\left[\partial_{i}^{u}, x_{i}\right]=\partial_{i}\left[\partial_{i}^{u-1}, x_{i}\right]+\left[\partial_{i}, x_{i}\right] \partial_{i}^{u-1}=\partial_{i}\left[\partial_{i}^{u-1}, x_{i}\right]+\partial_{i}^{u-1}
$$

Agora, usando a hipótese de indução, temos o resultado desejado:

$$
\left[\partial_{i}^{u}, x_{i}\right]=(u-1) \partial_{i} \partial_{i}^{u-2}+\partial_{i}^{u-1}=u \partial_{i}^{u-1}-\partial_{i}^{u-1}+\partial_{i}^{u-1}=u \partial_{i}^{u-1}
$$

4. Vamos considerar $l \in \mathbb{N}^{*}$, pois se $l=0$, não temos que provar nada. Vamos usar indução em $k$. O caso $k=0$ é trivial. Seja $k=1$.

$$
\sum_{j=0}^{1}\left(\begin{array}{l}
1 \\
j
\end{array}\right) \partial_{i}^{j}\left(x_{i}^{l}\right) \partial_{i}^{1-j}=x_{i}^{l} \partial_{i}+\partial_{i}\left(x_{i}^{l}\right)=x_{i}^{l} \partial_{i}+\partial x_{i}^{l} / \partial x_{i}=x_{i}^{l} \partial_{i}+l x_{i}^{l-1}
$$

Utilizando a relação descrita no item 2 temos que:

$$
\sum_{j=0}^{1}\left(\begin{array}{l}
1 \\
j
\end{array}\right) \partial_{i}^{j}\left(x_{i}^{l}\right) \partial_{i}^{1-j}=\partial_{i} x_{i}^{l}
$$

Assim o caso $k=1$ está verificado. Seja $k>1$. Suponhamos que o resultado é válido para $k-1$. Vamos verificar que também é válido para $k$. Temos que $\partial_{i}^{k} x_{i}^{l}=\partial_{i} \partial_{i}^{k-1} x_{i}^{l}$. Usando a hipótese de indução temos que:

$$
\partial_{i} \partial_{i}^{k-1} x_{i}^{l}=\partial_{i} \sum_{j=0}^{k-1}\left(\begin{array}{c}
k-1 \\
j
\end{array}\right) \partial_{i}^{j}\left(x_{i}^{l}\right) \partial_{i}^{k-1-j}=\sum_{j=0}^{k-1}\left(\begin{array}{c}
k-1 \\
j
\end{array}\right) \partial_{i}\left(\partial_{i}^{j}\left(x_{i}^{l}\right) \partial_{i}^{k-1-j}\right)
$$

Agora usando a regra de derivação do produto temos:

$$
\partial_{i}^{k} x_{i}^{l}=\sum_{j=0}^{k-1}\left(\begin{array}{c}
k-1 \\
j
\end{array}\right) \partial_{i}\left(\partial_{i}^{j}\left(x_{i}^{l}\right) \partial_{i}^{k-1-j}\right)=\sum_{j=0}^{k-1}\left(\begin{array}{c}
k-1 \\
j
\end{array}\right)\left(\partial_{i}^{j+1}\left(x_{i}^{l}\right) \partial_{i}^{k-1-j}+\partial_{i}^{j}\left(x_{i}^{l}\right) \partial_{i}^{k-j}\right)
$$

Então:

$$
\begin{aligned}
\partial_{i}^{k} x_{i}^{l} & =\sum_{j=0}^{k-1}\left(\begin{array}{c}
k-1 \\
j
\end{array}\right) \partial_{i}^{j}\left(x_{i}^{l}\right) \partial_{i}^{k-j}+\sum_{j=0}^{k-1}\left(\begin{array}{c}
k-1 \\
j
\end{array}\right) \partial_{i}^{j+1}\left(x_{i}^{l}\right) \partial_{i}^{k-1-j} \\
& =\sum_{j=0}^{k-1}\left(\begin{array}{c}
k-1 \\
j
\end{array}\right) \partial_{i}^{j}\left(x_{i}^{l}\right) \partial_{i}^{k-j}+\sum_{j=1}^{k}\left(\begin{array}{c}
k-1 \\
j-1
\end{array}\right) \partial_{i}^{j}\left(x_{i}^{l}\right) \partial_{i}^{k-j}
\end{aligned}
$$

Portanto:

$$
\partial_{i}^{k} x_{i}^{l}=x_{i}^{l} \partial_{i}^{k}+\sum_{j=1}^{k-1}\left(\begin{array}{c}
k-1 \\
j
\end{array}\right) \partial_{i}^{j}\left(x_{i}^{l}\right) \partial_{i}^{k-j}+\sum_{j=1}^{k-1}\left(\begin{array}{c}
k-1 \\
j-1
\end{array}\right) \partial_{i}^{j}\left(x_{i}^{l}\right) \partial_{i}^{k-j}+\partial_{i}^{k}\left(x_{i}^{l}\right)
$$


Pela Fórmula de Stieffel $\left(\begin{array}{c}k \\ j\end{array}\right)=\left(\begin{array}{c}k-1 \\ j\end{array}\right)+\left(\begin{array}{c}k-1 \\ j-1\end{array}\right)$, temos:

$$
\begin{aligned}
\partial_{i}^{k} x_{i}^{l} & =x_{i}^{l} \partial_{i}^{k}+\sum_{j=1}^{k-1}\left[\left(\begin{array}{c}
k-1 \\
j
\end{array}\right)+\left(\begin{array}{c}
k-1 \\
j-1
\end{array}\right)\right] \partial_{i}^{j}\left(x_{i}^{l}\right) \partial_{i}^{k-j}+\partial_{i}^{k}\left(x_{i}^{l}\right) \\
& =\left(\begin{array}{c}
k \\
0
\end{array}\right) x_{i}^{l} \partial_{i}^{k}+\sum_{j=1}^{k-1}\left(\begin{array}{c}
k \\
j
\end{array}\right) \partial_{i}^{j}\left(x_{i}^{l}\right) \partial_{i}^{k-j}+\left(\begin{array}{c}
k \\
k
\end{array}\right) \partial_{i}^{k}\left(x_{i}^{l}\right)=\sum_{j=0}^{k}\left(\begin{array}{c}
k \\
j
\end{array}\right) \partial_{i}^{j}\left(x_{i}^{l}\right) \partial_{i}^{k-j}
\end{aligned}
$$

Assim temos o resultado desejado:

$$
\partial_{i}^{k} x_{i}^{l}=\sum_{j=0}^{k}\left(\begin{array}{l}
k \\
j
\end{array}\right) \partial_{i}^{j}\left(x_{i}^{l}\right) \partial_{i}^{k-j}
$$

Em particular:

$$
\partial_{i}^{k} x_{i}^{l}=x_{i}^{l} \partial_{i}^{k}+\sum_{j=1}^{k}\left(\begin{array}{c}
k \\
j
\end{array}\right) \partial_{i}^{j}\left(x_{i}^{l}\right) \partial_{i}^{k-j}
$$

Isto é,

$$
\left[\partial_{i}^{k}, x_{i}^{l}\right]=\sum_{j=1}^{k}\left(\begin{array}{l}
k \\
j
\end{array}\right) \partial_{i}^{j}\left(x_{i}^{l}\right) \partial_{i}^{k-j}
$$

\subsubsection{Forma canônica}

Agora vamos construir uma base para a $n$-ésima álgebra de Weyl $A_{n}$ como um $k$-espaço vetorial. Esta base é conhecida como base canônica. Se um elemento de $A_{n}$ está escrito como uma combinação linear dos elementos desta base então dizemos que ele está na forma canônica.

É mais fácil descrever a base canônica se usarmos uma notação de multi-índices. Um multi-índice $\alpha$ é um elemento de $\mathbb{N}^{n}$, ou seja, $\alpha=\left(\alpha_{1}, \ldots, \alpha_{n}\right)$. Sendo assim, $x^{\alpha}$ é o monômio $x_{1}^{\alpha_{1}} \ldots x_{n}^{\alpha_{n}}$. O grau deste monômio é o comprimento $|\alpha|$ do multi-índice $\alpha$, ou seja, $|\alpha|=$ $\alpha_{1}+\ldots+\alpha_{n}$. De maneira análoga $\partial^{\beta}$, com $\beta \in \mathbb{N}^{n}$, é o monômio $\partial_{1}^{\beta 1} \ldots \partial_{n}^{\beta_{n}}$. Observamos que o par $(\alpha, \beta)$, em que $\alpha, \beta \in \mathbb{N}^{n}$, é um multi-índice de $\mathbb{N}^{2 n}$, então faz sentido falar de seu comprimento, isto é, $|\alpha|+|\beta|=\alpha_{1}+\ldots+\alpha_{n}+\beta_{1}+\ldots+\beta_{n}$. O fatorial de um multi-índice $\alpha \in \mathbb{N}^{n}$ é definido por $\alpha !=\alpha_{1}$ ! ... $\alpha_{n}$ !. Denotaremos por $e_{i}$ o multi-índice em que todas as entradas são nulas, com exceção da $i$-ésima entrada, que é igual a 1.

Definição 1.1.2. Uma boa ordem $\prec e m \mathbb{N}^{n}$ é dita ordem monomial se é compatível com a soma: $\alpha \prec \beta$ implica $\alpha+\gamma \prec \beta+\gamma$ para todo $\gamma \in \mathbb{N}^{n}$.

Para qualquer ordem monomial $\prec$ em $\mathbb{N}^{n}$ temos que $0=(0, \ldots, 0) \prec \alpha$ para todo $\alpha \in \mathbb{N}^{n}$. Se $\alpha, \beta \in \mathbb{N}^{n}$ são tais que $\alpha_{i} \leq \beta_{i}$ para todo $i$, então $\alpha \prec \beta$. Podemos relacionar qualquer ordem $\prec$ em $\mathbb{N}^{n}$ com uma ordem (também denotada por $\prec$ ) no conjunto dos monômios $\left\{x^{\alpha} \mid \alpha \in \mathbb{N}^{n}\right\}$, ou seja, $x^{\alpha} \prec x^{\beta}$ se, e somente se, $\alpha \prec \beta$.

Definição 1.1.3. A ordem lexicográfica (denotada por $<_{l e x}$ ) em $\mathbb{N}^{n}$ é definida como segue:

$$
\left(\alpha_{1}, \ldots, \alpha_{n}\right)<_{l e x}\left(\beta_{1}, \ldots, \beta_{n}\right)
$$

se, e somente se, a primeira componente não nula de $\left(\alpha_{1}-\beta_{1}, \ldots, \alpha_{n}-\beta_{n}\right)$ é negativa. 
A ordem lexicográfica $<_{l e x}$ é uma ordem monomial. Seja $\alpha<_{l e x} \beta$, ou seja, a primeira componente não nula de $\left(\alpha_{1}-\beta_{1}, \ldots, \alpha_{n}-\beta_{n}\right)$ é negativa. Assim, para $\gamma \in \mathbb{N}^{n}$, a primeira componente não nula de $\left(\alpha_{1}+\gamma_{1}-\beta_{1}-\gamma_{1}, \ldots, \alpha_{n}+\gamma_{n}-\beta_{n}-\gamma_{n}\right)$ é negativa, ou seja, $\alpha+\gamma<_{\text {lex }} \beta+\gamma$.

Lema 1.1.1. Sejam $\sigma, \beta \in \mathbb{N}^{n}$ tais que $|\sigma| \leq|\beta|$. Então $\partial^{\beta}\left(x^{\sigma}\right)=\beta$ ! se $\sigma=\beta$, e 0 caso contrário.

Demonstração. Sejam $\sigma, \beta \in \mathbb{N}^{n}$ tais que $|\sigma| \leq|\beta|$. Temos que:

$$
\partial^{\beta}\left(x^{\sigma}\right)=\partial_{1}^{\beta_{1}} \ldots \partial_{n}^{\beta_{n}}\left(x_{1}^{\sigma_{1}} \ldots x_{n}^{\sigma_{n}}\right)=\frac{\partial^{\beta_{1}}}{\partial x_{1}^{\beta_{1}}} \ldots \frac{\partial^{\beta_{n}}}{\partial x_{n}^{\beta_{n}}}\left(x_{1}^{\sigma_{1}} \ldots x_{n}^{\sigma_{n}}\right)=\frac{\partial^{\beta_{1}} x_{1}^{\sigma_{1}}}{\partial x_{1}^{\beta_{1}}} \ldots \frac{\partial^{\beta_{n}} x_{n}^{\sigma_{n}}}{\partial x_{n}^{\beta_{n}}}
$$

Observamos que se $\sigma_{i}=0$, para algum $i$, então $\partial_{i}^{\beta_{i}}\left(x_{i}^{\sigma_{i}}\right)=\partial_{i}^{\beta_{i}}(1)=0$ e se $\beta_{i}=0$, para algum $i$, então $\partial_{i}^{\beta_{i}}\left(x_{i}^{\sigma_{i}}\right)=1\left(x_{i}^{\sigma_{i}}\right)=x_{i}^{\sigma_{i}}$. Logo se $\sigma_{i}=\beta_{i}=0$, então $\partial_{i}^{\beta_{i}}\left(x_{i}^{\sigma_{i}}\right)=1$.

Para $1 \leq i \leq n$, o monômio $x_{i}^{\sigma_{i}}$ só depende da variável $x_{i}$. Assim cada derivada parcial $\partial / \partial x_{i}$ corresponde à derivada ordinária $d / d x_{i}$.

Se $\sigma=\beta$, então $\sigma_{i}=\beta_{i}, 1 \leq i \leq n$. Assim, para todo $i$ :

$$
\frac{\partial^{\beta_{i}} x_{i}^{\sigma_{i}}}{\partial x_{i}^{\beta_{i}}}=\beta_{i} \cdot\left(\beta_{i}-1\right) \cdots 2 \cdot 1=\beta_{i} \text { ! }
$$

Então $\partial^{\beta}\left(x^{\sigma}\right)=\beta_{1} ! \ldots \beta_{n} ! \stackrel{\text { def. }}{=} \beta$ !.

Se $\sigma \neq \beta$, então $\sigma_{i} \neq \beta_{i}$, para no mínimo algum $i$. Como $\sigma_{i}, \beta_{i} \in \mathbb{N}$, temos que $\sigma_{i}>\beta_{i}$ ou $\sigma_{i}<\beta_{i}$. Se $\sigma_{i}>\beta_{i}$, afirmamos que existe $1 \leq j \leq n, j \neq i$, tal que $\sigma_{j}<\beta_{j}$. De fato: suponha que para todo $1 \leq j \leq n, j \neq i, \sigma_{j} \geq \beta_{j}$ e $\sigma_{i}>\beta_{i}$. Então $\sigma_{1}+\ldots+\widehat{\sigma}_{i}+\ldots+\sigma_{n} \geq$ $\beta_{1}+\ldots+\widehat{\beta}_{i}+\ldots+\beta_{n}$. Assim $\sigma_{1}+\ldots+\sigma_{i}+\ldots+\sigma_{n}>\beta_{1}+\ldots+\beta_{i}+\ldots+\beta_{n}$, ou seja, $|\sigma|>|\beta|$. Isso contradiz a escolha de $\sigma, \beta$ tais que $|\sigma| \leq|\beta|$.

Portanto $\sigma_{j}<\beta_{j}$, para algum $j$. Então $\frac{\partial^{\beta_{j}} x_{j}^{\sigma_{j}}}{\partial x_{j}^{\beta_{j}}}=0$. Resultando em $\partial^{\beta}\left(x^{\sigma}\right)=0$.

Proposição 1.1.2. O conjunto $C=\left\{x^{\alpha} \partial^{\beta} \mid \alpha, \beta \in \mathbb{N}^{n}\right\}$ é uma base de $A_{n}$ como um espaço vetorial sobre $k$.

Demonstração. Vamos verificar que o conjunto $C$ gera a álgebra $A_{n}$ como $k$-espaço vetorial. Considere um monômio nos geradores de $A_{n}$, isto é, $\lambda_{1} \cdots \lambda_{r}$, em que $\lambda_{j} \in$ $\left\{x_{1}, \ldots, x_{n}, \partial_{1}, \ldots, \partial_{n}\right\}, 1 \leq j \leq r$. Usando a relação $\left[\partial_{i}, x_{j}\right]=\delta_{i j}$ podemos escrevê-lo como uma soma de monômios, com coeficientes em $k$, em que todas as potências dos $x$ 's estão agrupadas à esquerda de todos os $\partial$ 's. Dessa forma o monômio $\lambda_{1} \cdots \lambda_{r}$ é escrito como uma combinação linear de elementos pertencentes à $C$. Portanto $C$ é um conjunto gerador de $A_{n}$, como $k$-espaço vetorial.

Agora vamos verificar que $C$ é linearmente independente sobre $k$. Seja $D$ uma combinação linear finita de elementos de $C$, isto é, $D=\sum_{\alpha, \beta} c_{\alpha \beta} x^{\alpha} \partial^{\beta}$, em que $c_{\alpha \beta} \in k$. Temos que mostrar que se algum $c_{\alpha \beta}$ é não nulo, então $D \neq 0$. Como $D$ é um operador linear de $k[X], D \neq 0$ se, e somente se, existe um polinômio $f$ para o qual $D(f) \neq 0$. Nós construiremos tal polinômio $f$.

Seja $\sigma \in \mathbb{N}^{n}$ o menor elemento, com respeito à ordem lexicográfica, que aparece como expoente de $\partial$ em $D$. Vamos calcular $D\left(x^{\sigma}\right)=\sum_{\alpha, \beta} c_{\alpha \beta} x^{\alpha} \partial^{\beta}\left(x^{\sigma}\right)$. Usando o Lema 1.1.1, temos que $D\left(x^{\sigma}\right)=\sigma$ ! $\sum_{\alpha} c_{\alpha \sigma} x^{\alpha}$, pois se $\sigma$ é estritamente menor que $\beta$, na ordem lexicográfica, então $\partial^{\beta}\left(x^{\sigma}\right)=0$. Pela escolha de $\sigma$, existe $\alpha \in \mathbb{N}^{n}$ tal que $c_{\alpha \sigma} \neq 0$. Temos que $D\left(x^{\sigma}\right)$ é não 
nulo, pois no mínimo um dos coeficientes $c_{\alpha \sigma}$ é não nulo. Portanto $f=x^{\sigma}$ é o polinômio procurado, ou seja, $D \neq 0$.

Dessa forma um operador $P \in A_{n}$, se escreve de forma única como uma combinação linear finita dos elementos da base $C$, ou seja, $P=\sum_{\alpha, \beta} c_{\alpha \beta} x^{\alpha} \partial^{\beta}$, em que $c_{\alpha \beta} \in k$. Logo, podemos escrever $P=\sum_{\beta} p_{\beta} \partial^{\beta}$, em que $p_{\beta}=\sum_{\alpha} c_{\alpha \beta} x^{\alpha} \in k\left[x_{1}, \ldots, x_{n}\right]=k[X]$.

Observação 1.1.1. Seja A uma álgebra associativa. Para quaisquer $u, v, w \in A$, temos:

1. $[v w, u]=v w u-u v w=v w u-u v w+v u w-v u w=v[w, u]+[v, u] w$.

2. $[u, v w]=u v w-v w u=u v w-v w u+v u w-v u w=v[u, w]+[u, v] w$.

Proposição 1.1.3. Sejam $\alpha, \beta, \sigma, \eta \in \mathbb{N}^{n}$. Então:

$$
\left[x^{\alpha} \partial^{\beta}, x^{\sigma} \partial^{\eta}\right]=x^{\alpha}\left[\partial^{\beta}, x^{\sigma}\right] \partial^{\eta}+x^{\sigma}\left[x^{\alpha}, \partial^{\eta}\right] \partial^{\beta} .
$$

Demonstração. Para demonstrar esse resultado, vamos utilizar a Observação 1.1.1.

$$
\begin{aligned}
& {\left[x^{\alpha} \partial^{\beta}, x^{\sigma} \partial^{\eta}\right] \stackrel{\text { Obs.1.1.1.1 }}{=} x^{\alpha}\left[\partial^{\beta}, x^{\sigma} \partial^{\eta}\right]+\left[x^{\alpha}, x^{\sigma} \partial^{\eta}\right] \partial^{\beta}} \\
& \text { Obs.1.1.1.2 } x^{\alpha}\left(x^{\sigma}\left[\partial^{\beta}, \partial^{\eta}\right]+\left[\partial^{\beta}, x^{\sigma}\right] \partial^{\eta}\right)+\left(x^{\sigma}\left[x^{\alpha}, \partial^{\eta}\right]+\left[x^{\alpha}, x^{\sigma}\right] \partial^{\eta}\right) \partial^{\beta}
\end{aligned}
$$

Como $\left[\partial^{\beta}, \partial^{\eta}\right]=\left[x^{\alpha}, x^{\sigma}\right]=0$, temos que

$$
\left[x^{\alpha} \partial^{\beta}, x^{\sigma} \partial^{\eta}\right]=x^{\alpha}\left[\partial^{\beta}, x^{\sigma}\right] \partial^{\eta}+x^{\sigma}\left[x^{\alpha}, \partial^{\eta}\right] \partial^{\beta} .
$$

Proposição 1.1.4. Seja $\beta \in \mathbb{N}^{n}$, com $\beta_{i} \neq 0$. Então $\left[\partial^{\beta}, x_{i}\right]=\beta_{i} \partial^{\beta-e_{i}}$.

Demonstração.

$$
\begin{aligned}
& {\left[\partial^{\beta}, x_{i}\right]=\left[\partial_{1}^{\beta_{1}} \ldots \partial_{n}^{\beta_{n}}, x_{i}\right]} \\
& =\partial_{1}^{\beta_{1}} \ldots \partial_{n}^{\beta_{n}} x_{i}-x_{i} \partial_{1}^{\beta_{1}} \ldots \partial_{n}^{\beta_{n}} \\
& =\partial_{1}^{\beta_{1}} \ldots \widehat{\partial_{i}^{\beta i}} \ldots \partial_{n}^{\beta_{n}} \partial_{i}^{\beta_{i}} x_{i}-\partial_{1}^{\beta_{1}} \ldots \widehat{\partial_{i}^{\beta i}} \ldots \partial_{n}^{\beta_{n}} x_{i} \partial_{i}^{\beta_{i}} \\
& =\partial_{1}^{\beta_{1}} \ldots \widehat{\partial_{i}^{\beta i}} \ldots \partial_{n}^{\beta_{n}}\left[\partial_{i}^{\beta_{i}}, x_{i}\right] \\
& \stackrel{\text { Prop.1.1.1.3 }}{=} \partial_{1}^{\beta_{1}} \ldots \widehat{\partial_{i}^{\beta i}} \ldots \partial_{n}^{\beta_{n}} \beta_{i} \partial_{i}^{\beta_{i}-1} \\
& =\beta_{i} \partial^{\beta-e_{i}}
\end{aligned}
$$

Proposição 1.1.5. Sejam $\alpha, \beta \in \mathbb{N}^{n}, p \in k[X]$ e $c_{\alpha \beta} \in k$. Então para $1 \leq i \leq n$ :

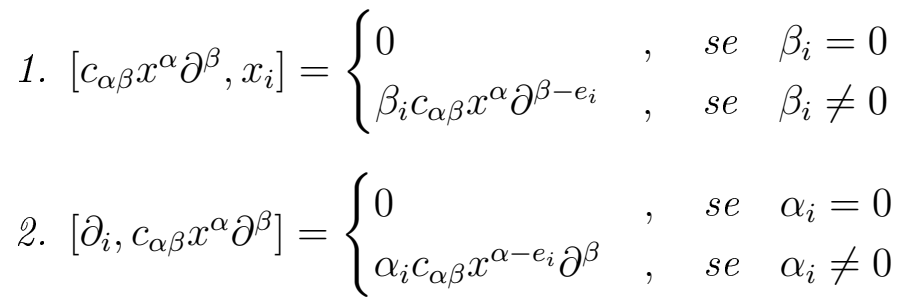

3. $\left[\partial^{\beta}, p\right]=\partial_{i}\left[\partial^{\beta-e_{i}}, p\right]+\left[\partial_{i}, p\right] \partial^{\beta-e_{i}}$, desde que $\beta_{i} \neq 0$. 
Demonstração. 1. $\left[c_{\alpha \beta} x^{\alpha} \partial^{\beta}, x_{i}\right]=c_{\alpha \beta}\left[x^{\alpha} \partial^{\beta}, x_{i} \cdot 1\right]$. Pela Proposição 1.1.3, temos que

$$
c_{\alpha \beta}\left[x^{\alpha} \partial^{\beta}, x_{i} \cdot 1\right]=c_{\alpha \beta}\left(x^{\alpha}\left[\partial^{\beta}, x_{i}\right] 1+x_{i}\left[x^{\alpha}, 1\right] \partial^{\beta}\right)=c_{\alpha \beta} x^{\alpha}\left[\partial^{\beta}, x_{i}\right]
$$

Se $\beta_{i}=0$, então $\left[\partial^{\beta}, x_{i}\right]=0$, ou seja, $\left[c_{\alpha \beta} x^{\alpha} \partial^{\beta}, x_{i}\right]=0$. Se $\beta_{i} \neq 0$, pela Proposição 1.1.4, $\left[\partial^{\beta}, x_{i}\right]=\beta_{i} \partial^{\beta-e_{i}}$. Assim $\left[c_{\alpha \beta} x^{\alpha} \partial^{\beta}, x_{i}\right]=\beta_{i} c_{\alpha \beta} x^{\alpha} \partial^{\beta-e_{i}}$.

2. $\left[\partial_{i}, c_{\alpha \beta} x^{\alpha} \partial^{\beta}\right]=c_{\alpha \beta}\left[1 \cdot \partial_{i}, x^{\alpha} \partial^{\beta}\right]$. Pela Proposição 1.1.3, temos que

$$
c_{\alpha \beta}\left[1 \cdot \partial_{i}, x^{\alpha} \partial^{\beta}\right]=c_{\alpha \beta}\left(1\left[\partial_{i}, x^{\alpha}\right] \partial^{\beta}+x^{\alpha}\left[1, \partial^{\beta}\right] \partial_{i}\right)=c_{\alpha \beta}\left[\partial_{i}, x^{\alpha}\right] \partial^{\beta}
$$

Se $\alpha_{i}=0$, então $\left[\partial_{i}, x^{\alpha}\right]=0$, ou seja, $\left[\partial_{i}, c_{\alpha \beta} x^{\alpha} \partial^{\beta}\right]=0$. Se $\alpha_{i} \neq 0$, pela Proposição 1.1.1, item 1, $\left[\partial_{i}, x^{\alpha}\right]=\alpha_{i} x^{\alpha-e_{i}}$. Assim $\left[\partial_{i}, c_{\alpha \beta} x^{\alpha} \partial^{\beta}\right]=\alpha_{i} c_{\alpha \beta} x^{\alpha-e_{i}} \partial^{\beta}$.

3. Um polinômio $p \in k[X]$ tem a forma $p=\sum_{\alpha} c_{\alpha} x^{\alpha}$, com $c_{\alpha} \in k$. Então $\left[\partial^{\beta}, p\right]=$ $\sum_{\alpha} c_{\alpha}\left[\partial^{\beta}, x^{\alpha}\right]$. Considerando $\beta_{i} \neq 0$, temos que $\sum_{\alpha} c_{\alpha}\left[\partial^{\beta}, x^{\alpha}\right]=\sum_{\alpha} c_{\alpha}\left[\partial_{i} \partial^{\beta-e_{i}}, x^{\alpha}\right]$. Pela Observação 1.1.1, $\left[\partial_{i} \partial^{\beta-e_{i}}, x^{\alpha}\right]=\partial_{i}\left[\partial^{\beta-e_{i}}, x^{\alpha}\right]+\left[\partial_{i}, x^{\alpha}\right] \partial^{\beta-e_{i}}$. Então:

$$
\sum_{\alpha} c_{\alpha}\left[\partial^{\beta}, x^{\alpha}\right]=\sum_{\alpha} c_{\alpha}\left[\partial_{i} \partial^{\beta-e_{i}}, x^{\alpha}\right]=\partial_{i}\left[\partial^{\beta-e_{i}}, \sum_{\alpha} c_{\alpha} x^{\alpha}\right]+\left[\partial_{i}, \sum_{\alpha} c_{\alpha} x^{\alpha}\right] \partial^{\beta-e_{i}}
$$

Portanto

$$
\left[\partial^{\beta}, p\right]=\partial_{i}\left[\partial^{\beta-e_{i}}, p\right]+\left[\partial_{i}, p\right] \partial^{\beta-e_{i}}
$$

\subsubsection{Geradores e relações}

Outra forma de definir a álgebra de Weyl é por geradores e relações. Esta maneira é mais direta e explícita. Mais precisamente, nós podemos definir a álgebra de Weyl como um quociente de uma $k$-álgebra livre com $2 n$ geradores por um ideal de relações.

Definição 1.1 .4 (k-álgebra livre). Seja $\left\{x_{i} \mid i \in I\right\}$ um conjunto não vazio. O conjunto $k\left\langle x_{i} \mid i \in I\right\rangle$ é uma k-álgebra, em que o produto de dois monômios é a justaposição simples e $\lambda x_{i}=x_{i} \lambda$, para todo $i \in I$ e para todo $\lambda \in k$. Os elementos de $k\left\langle x_{i} \mid i \in I\right\rangle$ são "polinômios" com coeficientes em $k$ com variáveis não comutativas $\left\{x_{i} \mid i \in I\right\} . k\left\langle x_{i} \mid i \in I\right\rangle$ é uma $k$ álgebra associativa livre.

Teorema 1.1.1 (Propriedade universal). Sejam I um conjunto não vazio e A uma k-álgebra. Seja:

$$
\begin{aligned}
f: I & \longrightarrow A \\
i & \longmapsto a_{i}
\end{aligned}
$$

uma função. Então existe um único homomorfismo de k-álgebras $\varphi: k\left\langle x_{i} \mid i \in I\right\rangle \longrightarrow A$ tal que $\varphi\left(x_{i}\right)=a_{i}$, para todo $i \in I$.

Definição 1.1.5. Seja $k\left\langle y_{1}, \ldots, y_{n}, z_{1}, \ldots, z_{n}\right\rangle$ a $k_{\text {-álgebra associativa livre nos geradores }}$ $y_{1}, \ldots, y_{n}, z_{1}, \ldots, z_{n}$. Seja $J$ o ideal gerado pelos elementos $\left[y_{i}, y_{j}\right],\left[z_{i}, z_{j}\right]$ e $\left[z_{i}, y_{j}\right]-\delta_{i j}$, $1 \leq i, j \leq n$. Seja $A_{n}^{*}(k)$ o quociente da k-álgebra associativa livre pelo ideal J.

A $k$-álgebra associativa livre $k\left\langle y_{1}, \ldots, y_{n}, z_{1}, \ldots, z_{n}\right\rangle$ é o conjunto de todas as combinações lineares finitas de palavras em $y_{1}, \ldots, y_{n}, z_{1}, \ldots, z_{n}$ sobre $k$. A multiplicação de dois monômios 
é a justaposição simples, de acordo com a definição. Temos também que $A_{n}(k)$ (a $n$-ésima álgebra de Weyl) é uma $k$-álgebra associativa livre, gerada por $x_{1}, \ldots, x_{n}, \partial_{1}, \ldots, \partial_{n}$.

Podemos definir o homomorfismo sobrejetivo de $k$-álgebras associativas

$$
\phi: k\left\langle y_{1}, \ldots, y_{n}, z_{1}, \ldots, z_{n}\right\rangle \longrightarrow A_{n}(k)
$$

por $\phi\left(y_{i}\right)=x_{i}$ e $\phi\left(z_{i}\right)=\partial_{i}, 1 \leq i \leq n$, de acordo com o Teorema 1.1.1.

O ideal $J$ gerado pelos elementos $\left[y_{i}, y_{j}\right],\left[z_{i}, z_{j}\right]$ e $\left[z_{i}, y_{j}\right]-\delta_{i j}, 1 \leq i, j \leq n$, está contido no kernel de $\phi$, isto é, $J \subseteq K e r \phi$, por conta das relações satisfeitas pelos geradores da $n$ ésima álgebra de Weyl $A_{n}(k)$. Então $\phi$ induz um homomorfismo de $k$-álgebras associativas $\hat{\phi}: A_{n}^{*}(k) \longrightarrow A_{n}(k)$, tal que $\hat{\phi}(w+J)=\phi(w)$ para todo $w \in k\left\langle y_{1}, \ldots, y_{n}, z_{1}, \ldots, z_{n}\right\rangle$.

Teorema 1.1.2. $\hat{\phi}: A_{n}^{*}(k) \longrightarrow A_{n}$ é um isomorfismo.

Demonstração. Exatamente como na demonstração da Proposição 1.1.2, podemos usar as relações entre as classes $y_{i}+J, z_{i}+J, 1 \leq i \leq n$, para verificar que todo elemento de $A_{n}^{*}(k)$ pode ser escrito como uma combinação linear de monômios da forma

$$
y_{1}^{m_{1}} \ldots y_{n}^{m_{n}} z_{1}^{p_{1}} \ldots z_{n}^{p_{n}}+J
$$

em que $m=\left(m_{1}, \ldots, m_{n}\right), p=\left(p_{1}, \ldots, p_{n}\right) \in \mathbb{N}^{n}$. Pela Proposição 1.1.2, as imagens destes monômios sob $\hat{\phi}$ formam uma base de $A_{n}(k)$ como um espaço vetorial sobre $k$. Portanto, em particular, os monômios devem ser linearmente independentes em $A_{n}^{*}(k)$. Assim, esse homomorfismo é um isomorfismo de $k$-espaços vetoriais, e portanto, de $k$-álgebras.

Vamos mostrar um importante exemplo da relação entre uma $k$-álgebra associativa livre unital e a $n$-ésima álgebra de Weyl $A_{n}(k)$.

Exemplo 1.1.1. A n-ésima álgebra de Heisenberg $H_{n}$ é uma álgebra de Lie dada por geradores $P_{1}, \ldots, P_{n}, Q_{1}, \ldots, Q_{n}, C$ que satisfazem as seguintes relações:

$$
\left[P_{i}, P_{j}\right]=\left[Q_{i}, Q_{j}\right]=0 \quad, \quad\left[Q_{i}, P_{j}\right]=\delta_{i j} C
$$

com $1 \leq i, j \leq n$, em que $C$ está no centro. $A_{n}(k)$ é imagem homomórfica de $U\left(H_{n}\right)$ (álgebra envolvente universal da n-ésima álgebra de Heisenberg), pois podemos definir um epimorfismo $\phi: U\left(H_{n}\right) \rightarrow A_{n}$, tal que $\phi\left(P_{i}\right)=x_{i}, \phi\left(Q_{i}\right)=\partial_{i}, 1 \leq i \leq n$. Portanto, $A_{n}(k) e ́$ o quociente de $U\left(H_{n}\right)$ pelo ideal gerado por $C-1$.

\subsubsection{O grau de um operador em $A_{n}$}

O grau de um operador de $A_{n}$ comporta-se, em muitos aspectos, como o grau de um polinômio. As diferenças são contabilizadas pela não comutatividade de $A_{n}$.

Definição 1.1.6. Seja $D \in A_{n}$. $O$ grau de $D$ é o maior comprimento dos multi-índices $(\alpha, \beta) \in \mathbb{N}^{n} \times \mathbb{N}^{n}$, para qual $x^{\alpha} \partial^{\beta}$ aparece com coeficiente não nulo na forma canônica de $D$. É denotado por deg $(D)$. Se $D$ é o operador nulo, por convenção, deg $(D)=-\infty$.

Exemplo 1.1.2. Seja $P=2 x_{1} \partial_{2}+x_{1} x_{2}^{3} \partial_{1} \partial_{2} \in A_{2}$. Temos que $\operatorname{deg}(P)=6$.

Teorema 1.1.3. Para $D, D^{\prime} \in A_{n}$, o grau satisfaz as seguintes propriedades:

1. $\operatorname{deg}\left(D+D^{\prime}\right) \leq \max \left\{\operatorname{deg}(D), \operatorname{deg}\left(D^{\prime}\right)\right\}$

2. $\operatorname{deg}\left(D D^{\prime}\right)=\operatorname{deg}(D)+\operatorname{deg}\left(D^{\prime}\right)$. 
3. $\operatorname{deg}\left[D, D^{\prime}\right] \leq \operatorname{deg}(D)+\operatorname{deg}\left(D^{\prime}\right)-2$.

Demonstração. Se $D, D^{\prime} \in A_{n}$, estão escritos na forma canônica, então $D+D^{\prime}$ também está. Então concluímos que $\operatorname{deg}\left(D+D^{\prime}\right) \leq \max \left\{\operatorname{deg}(D), \operatorname{deg}\left(D^{\prime}\right)\right\}$. Notamos que se $\operatorname{deg}(D) \neq$ $\operatorname{deg}\left(D^{\prime}\right)$ então temos a igualdade, ou seja, $\operatorname{deg}\left(D+D^{\prime}\right)=\max \left\{\operatorname{deg}(D), \operatorname{deg}\left(D^{\prime}\right)\right\}$.

Vamos usar indução sobre $\operatorname{deg}(D)+\operatorname{deg}\left(D^{\prime}\right)$ para provar os itens 2 e 3. Se $\operatorname{deg}(D)$ ou $\operatorname{deg}\left(D^{\prime}\right)$ é zero, então $D$ ou $D^{\prime}$ é constante. Sem perda de generalidade vamos supor que $D$ é uma constante. Temos que $\operatorname{deg}\left(D D^{\prime}\right)=\operatorname{deg}\left(D^{\prime}\right)=0+\operatorname{deg}\left(D^{\prime}\right)=\operatorname{deg}(D)+\operatorname{deg}\left(D^{\prime}\right)$. Da mesma forma $\left[D, D^{\prime}\right]=0$. Assim $\operatorname{deg}\left[D, D^{\prime}\right]=-\infty \leq \operatorname{deg}(D)+\operatorname{deg}\left(D^{\prime}\right)-2$.

Suponhamos que $\operatorname{deg}(D), \operatorname{deg}\left(D^{\prime}\right) \geq 1$ e que o resultado é válido quando $\operatorname{deg}(D)+$ $\operatorname{deg}\left(D^{\prime}\right)<k$. Escolhemos $D, D^{\prime} \in A_{n}$, tal que $\operatorname{deg}(D)+\operatorname{deg}\left(D^{\prime}\right)=k$. Pelo item 1 é suficiente provar os itens 2 e 3 quando $D, D^{\prime}$ são monômios. Suponhamos primeiramente que $D=\partial^{\beta}$ e $D^{\prime}=x^{\alpha} \operatorname{com}|\alpha|+|\beta|=k$. Se $\beta_{i} \neq 0$, então pela Proposição 1.1.5, item 3, temos que:

$$
\left[\partial^{\beta}, x^{\alpha}\right]=\partial_{i}\left[\partial^{\beta-e_{i}}, x^{\alpha}\right]+\left[\partial_{i}, x^{\alpha}\right] \partial^{\beta-e_{i}}
$$

Por indução, temos que:

$$
\operatorname{deg}\left[\partial^{\beta-e_{i}}, x^{\alpha}\right] \leq|\alpha|+|\beta|-1-2=|\alpha|+|\beta|-3
$$

pois $\left|\beta-e_{i}\right|=|\beta|-1$. Temos também:

$$
\operatorname{deg}\left[\partial_{i}, x^{\alpha}\right] \leq|\alpha|+1-2=|\alpha|-1
$$

Então, usando a hipótese de indução novamente (pois $\operatorname{deg}\left(\partial_{i}\right)+\operatorname{deg}\left[\partial^{\beta-e_{i}}, x^{\alpha}\right]<k$ e $\operatorname{deg}\left[\partial_{i}, x^{\alpha}\right]$ $\left.+\operatorname{deg}\left(\partial^{\beta-e_{i}}\right)<k\right)$ concluímos que:

$$
\operatorname{deg}\left(\partial_{i}\left[\partial^{\beta-e_{i}}, x^{\alpha}\right]\right)=\operatorname{deg}\left(\partial_{i}\right)+\operatorname{deg}\left[\partial^{\beta-e_{i}}, x^{\alpha}\right] \leq 1+|\alpha|+|\beta|-3=|\alpha|+|\beta|-2
$$

e

$$
\operatorname{deg}\left(\left[\partial_{i}, x^{\alpha}\right] \partial^{\beta-e_{i}}\right)=\operatorname{deg}\left[\partial_{i}, x^{\alpha}\right]+\operatorname{deg}\left(\partial^{\beta-e_{i}}\right) \leq|\alpha|-1+|\beta|-1=|\alpha|+|\beta|-2
$$

Portanto

$$
\begin{aligned}
\operatorname{deg}\left[\partial^{\beta}, x^{\alpha}\right] & =\operatorname{deg}\left(\partial_{i}\left[\partial^{\beta-e_{i}}, x^{\alpha}\right]+\left[\partial_{i}, x^{\alpha}\right] \partial^{\beta-e_{i}}\right) \\
& \leq \max \left\{\operatorname{deg}\left(\partial_{i}\left[\partial^{\beta-e_{i}}, x^{\alpha}\right]\right), \operatorname{deg}\left(\left[\partial_{i}, x^{\alpha}\right] \partial^{\beta-e_{i}}\right)\right\} \\
& \leq|\alpha|+|\beta|-2=\operatorname{deg}\left(x^{\alpha}\right)+\operatorname{deg}\left(\partial^{\beta}\right)-2
\end{aligned}
$$

Assim $\operatorname{deg}\left[D, D^{\prime}\right] \leq \operatorname{deg}(D)+\operatorname{deg}\left(D^{\prime}\right)-2$, portanto vale o item 3. Sabemos que $\left[\partial^{\beta}, x^{\alpha}\right]=$ $\partial^{\beta} x^{\alpha}-x^{\alpha} \partial^{\beta}$. Temos que $\operatorname{deg}\left(x^{\alpha} \partial^{\beta}\right) \neq \operatorname{deg}\left[\partial^{\beta}, x^{\alpha}\right]$, pois $\operatorname{deg}\left(x^{\alpha} \partial^{\beta}\right)=|\alpha|+|\beta|$ e $\operatorname{deg}\left[\partial^{\beta}, x^{\alpha}\right] \leq$ $|\alpha|+|\beta|-2$. Então:

$$
\operatorname{deg}\left(\partial^{\beta} x^{\alpha}\right)=\operatorname{deg}\left(\left[\partial^{\beta}, x^{\alpha}\right]+x^{\alpha} \partial^{\beta}\right)=\max \left\{\operatorname{deg}\left[\partial^{\beta}, x^{\alpha}\right], \operatorname{deg}\left(x^{\alpha} \partial^{\beta}\right)\right\}=|\alpha|+|\beta|
$$

Assim $\operatorname{deg}\left(D D^{\prime}\right)=\operatorname{deg}(D)+\operatorname{deg}\left(D^{\prime}\right)$, isto é, vale o item 2.

Agora sejam $D=x^{\sigma} \partial^{\beta}$ e $D^{\prime}=x^{\alpha} \partial^{\eta}$, em que $|\sigma|+|\beta|+|\alpha|+|\eta|=k$. Se $|\alpha|=|\beta|=0$, o resultado é óbvio. Então suponhamos que este não seja o caso. Temos que $\partial^{\beta} x^{\alpha}=x^{\alpha} \partial^{\beta}+P$, tal que $P=\left[\partial^{\beta}, x^{\alpha}\right]$ e sabemos pelo caso anterior que $\operatorname{deg}\left[\partial^{\beta}, x^{\alpha}\right] \leq|\alpha|+|\beta|-2$. Assim:

$$
D D^{\prime}=x^{\sigma} \partial^{\beta} x^{\alpha} \partial^{\eta}=x^{\sigma}\left(\partial^{\beta} x^{\alpha}\right) \partial^{\eta}=x^{\sigma}\left(x^{\alpha} \partial^{\beta}+P\right) \partial^{\eta}=x^{\sigma+\alpha} \partial^{\beta+\eta}+x^{\sigma} P \partial^{\eta}
$$


Como $\operatorname{deg}\left(x^{\sigma}\right)+\operatorname{deg}(P)+\operatorname{deg}\left(\partial^{\eta}\right) \leq|\sigma|+|\beta|+|\alpha|+|\eta|-2<k$, pela hipótese de indução: $\operatorname{deg}\left(x^{\sigma} P \partial^{\eta}\right)=\operatorname{deg}\left(x^{\sigma}\right)+\operatorname{deg}(P)+\operatorname{deg}\left(\partial^{\eta}\right) \leq|\sigma|+|\beta|+|\alpha|+|\eta|-2=\operatorname{deg}(D)+\operatorname{deg}\left(D^{\prime}\right)-2$

Ou seja, $\operatorname{deg}\left(x^{\sigma} P \partial^{\eta}\right) \leq \operatorname{deg}(D)+\operatorname{deg}\left(D^{\prime}\right)-2$. Observamos que $\operatorname{deg}\left(x^{\sigma+\alpha} \partial^{\beta+\eta}\right)=|\sigma|+|\beta|+$ $|\alpha|+|\eta|=\operatorname{deg}(D)+\operatorname{deg}\left(D^{\prime}\right)$. Portanto $\operatorname{deg}\left(x^{\sigma+\alpha} \partial^{\beta+\eta}\right) \neq \operatorname{deg}\left(x^{\sigma} P \partial^{\eta}\right)$. Então:

$$
\begin{aligned}
\operatorname{deg}\left(D D^{\prime}\right) & =\operatorname{deg}\left(x^{\sigma+\alpha} \partial^{\beta+\eta}+x^{\sigma} P \partial^{\eta}\right) \\
& =\max \left\{\operatorname{deg}\left(x^{\sigma+\alpha} \partial^{\beta+\eta}\right), \operatorname{deg}\left(x^{\sigma} P \partial^{\eta}\right)\right\} \\
& =\operatorname{deg}\left(x^{\sigma+\alpha} \partial^{\beta+\eta}\right)=|\sigma|+|\beta|+|\alpha|+|\eta| \\
& =\operatorname{deg}(D)+\operatorname{deg}\left(D^{\prime}\right)
\end{aligned}
$$

Ou seja, $\operatorname{deg}\left(D D^{\prime}\right)=\operatorname{deg}(D)+\operatorname{deg}\left(D^{\prime}\right)$, isto é, vale o item 2. Os cálculos acima nos mostram que $D D^{\prime}=x^{\sigma+\alpha} \partial^{\beta+\eta}+Q_{1}$, em que $\operatorname{deg}\left(Q_{1}\right) \leq \operatorname{deg}(D)+\operatorname{deg}\left(D^{\prime}\right)-2$. Similarmente, temos que $D^{\prime} D=x^{\sigma+\alpha} \partial^{\beta+\eta}+Q_{2}$, em que $\operatorname{deg}\left(Q_{2}\right) \leq \operatorname{deg}(D)+\operatorname{deg}\left(D^{\prime}\right)-2$. Então $\left[D, D^{\prime}\right]=$ $D D^{\prime}-D^{\prime} D=Q_{1}-Q_{2}$. Portanto $\operatorname{deg}\left[D, D^{\prime}\right]=\operatorname{deg}\left(Q_{1}-Q_{2}\right) \leq \operatorname{deg}(D)+\operatorname{deg}\left(D^{\prime}\right)-2$, vale o item 3. Assim concluímos a demonstração.

Como no caso do anel de polinômios sobre um corpo, o Teorema 1.1.3 pode ser usado para provar o seguinte resultado:

Corolário 1.1.1. A álgebra $A_{n}$ é um domínio.

Demonstração. Sejam $D, D^{\prime} \in A_{n}$, dois operadores não nulos. Podemos supor sem perda de generalidade que $D, D^{\prime}$ estão escritos na forma canônica.

Temos que $\operatorname{deg}(D), \operatorname{deg}\left(D^{\prime}\right) \geq 0$. Pelo Teorema 1.1.3, item 2, $\operatorname{deg}\left(D D^{\prime}\right)=\operatorname{deg}(D)+$ $\operatorname{deg}\left(D^{\prime}\right) \geq 0$. Então $D D^{\prime} \neq 0$ e portanto $A_{n}$ é um domínio.

Um anel comutativo simples é um corpo, mas isso não é verdade para anéis não comutativos. A álgebra de Weyl $A_{n}$ é um anel simples, mas está muito longe de ser um anel de divisão.

Teorema 1.1.4. A álgebra $A_{n}$ é simples.

Demonstração. Seja $I$ um ideal bilateral não nulo de $A_{n}$. Seja $D \neq 0$, um elemento com menor grau em $I$. Se $D$ tem grau 0, ele é uma constante. Assim $A_{n} D=D A_{n}=A_{n} \subseteq I$. Portanto $I=A_{n}$.

Agora vamos assumir que $D$ tem grau $k>0$ e chegaremos em uma contradição. Suponha que $(\alpha, \beta)$ é um multi-índice de comprimento $k$. Seja $x^{\alpha} \partial^{\beta}$ um somando de $D$ com coeficiente não nulo, em que $\beta_{i} \neq 0$. Vamos analisar o elemento $\left[x_{i}, D\right]$. $D=a_{\alpha \beta} x^{\alpha} \partial^{\beta}+D^{\prime}$, em que $\operatorname{deg}\left(D^{\prime}\right)<k$. Assim $\left[x_{i}, D\right]=\left[x_{i}, a_{\alpha \beta} x^{\alpha} \partial^{\beta}\right]+\left[x_{i}, D^{\prime}\right]$. Temos que:

$$
\left[x_{i}, a_{\alpha \beta} x^{\alpha} \partial^{\beta}\right] \stackrel{\text { Prop.1.1.5.1 }}{=}-\beta_{i} a_{\alpha \beta} x^{\alpha} \partial^{\beta-e_{i}}
$$

Então $\operatorname{deg}\left(\left[x_{i}, a_{\alpha \beta} x^{\alpha} \partial^{\beta}\right]\right)=\operatorname{deg}\left(-\beta_{i} a_{\alpha \beta} x^{\alpha} \partial^{\beta-e_{i}}\right)=|\alpha|+|\beta|-1=k-1$. Por outro lado, pelo Teorema 1.1.3, item $3, \operatorname{deg}\left[x_{i}, D^{\prime}\right] \leq 1+\operatorname{deg}\left(D^{\prime}\right)-2=\operatorname{deg}\left(D^{\prime}\right)-1<k-1$. Como $\operatorname{deg}\left(\left[x_{i}, a_{\alpha \beta} x^{\alpha} \partial^{\beta}\right]\right) \neq \operatorname{deg}\left[x_{i}, D^{\prime}\right]$, pelo Teorema 1.1.3, item $1, \operatorname{deg}\left[x_{i}, D\right]=k-1$. Como $k>0$, $\operatorname{deg}\left[x_{i}, D\right] \geq 0$ e assim $\left[x_{i}, D\right]$ é não nulo.

Como $I$ é um ideal bilateral de $A_{n}$ e $D \neq 0, D \in I$, temos que $\left[x_{i}, D\right]=x_{i} D-D x_{i} \in$ I. Mas isso contradiz a minimalidade de $D$. Então $\beta=(0, \ldots, 0)$ e $|\alpha|=k$. Como $k>0$, devemos ter $\alpha_{i} \neq 0$, para algum $i=1,2, \ldots, n$. Agora vamos analisar o elemento $\left[\partial_{i}, D\right]$. 
Seja $D=a_{\alpha} x^{\alpha}+D^{\prime}$, em que $\operatorname{deg}\left(D^{\prime}\right)<k$. Assim $\left[\partial_{i}, D\right]=a_{\alpha}\left[\partial_{i}, x^{\alpha}\right]+\left[\partial_{i}, D^{\prime}\right]$. Temos que:

$$
\left[\partial_{i}, x^{\alpha}\right] \stackrel{\text { Prop.1.1.1.1 }}{=} \alpha_{i} x^{\alpha-e_{i}}
$$

Temos que $\operatorname{deg}\left(a_{\alpha}\left[\partial_{i}, x^{\alpha}\right]\right)=\operatorname{deg}\left(a_{\alpha} \alpha_{i} x^{\alpha-e_{i}}\right)=|\alpha|-1=k-1$. Pelo Teorema 1.1.3, item $3, \operatorname{deg}\left[\partial_{i}, D^{\prime}\right] \leq 1+\operatorname{deg}\left(D^{\prime}\right)-2=\operatorname{deg}\left(D^{\prime}\right)-1<k-1$. Então pelo Teorema 1.1.3, item 1 , concluímos que $\operatorname{deg}\left[\partial_{i}, D\right]=k-1$ e como $k>0$, $\left[\partial_{i}, D\right]$ é um elemento não nulo. Como $D \in I$ é não nulo e $I$ é um ideal bilateral de $A_{n},\left[\partial_{i}, D\right] \in I$. Isso novamente contradiz a minimalidade de $D$.

Portanto se $I$ é um ideal bilateral de $A_{n}$ não nulo, $I=A_{n}$, ou seja, $A_{n}$ é simples.

O kernel de um endomorfismo de $A_{n}$ é um ideal bilateral. Então temos o seguinte Corolário:

Corolário 1.1.2. Todo endomorfismo não nulo de $A_{n}$ é injetivo.

Embora $A_{n}$ não tenha nenhum ideal bilateral além dos triviais, $A_{n}$ não é um anel de divisão. De fato, os únicos elementos de $A_{n}$ que possuem inversos são as constantes.

Proposição 1.1.6. As unidades da álgebra de Weyl $A_{n}$ são as constantes.

Demonstração. Vamos demonstrar esse fato usando o grau de um operador.

Seja $D \in A_{n}$ um operador não nulo. Se $D$ possui um inverso, então existe $D^{\prime} \in A_{n}$, não nulo, tal que $D D^{\prime}=1$, em que 1 é o operador identidade. Calculando os graus, pelo Teorema 1.1.3, item 2, obtemos $\operatorname{deg}(D)+\operatorname{deg}\left(D^{\prime}\right)=0$. Como o grau de um operador não nulo é sempre não negativo, temos que $\operatorname{deg}(D), \operatorname{deg}\left(D^{\prime}\right) \geq 0$. Então concluímos que $\operatorname{deg}(D)=\operatorname{deg}\left(D^{\prime}\right)=0$. Portanto $D$ é uma constante, isto é, $D \in k$.

Então todo operador não constante de $A_{n}$ gera um ideal à esquerda não trivial. Porém, a álgebra de Weyl não é um anel de ideais principais à esquerda. Por exemplo, o ideal à esquerda gerado por $\partial_{1}, \partial_{2}$ em $A_{2}$ não é principal (veja [Cou95], Capítulo 2, Exercício 4.1). Entretanto, todo ideal à esquerda de $A_{n}$ é gerado por dois elementos. Este é um importante resultado atribuído à J. T. Stafford. A prova é muito técnica e pode ser encontrada no artigo original de Stafford [Sta78] e também em [Bjö79].

\subsubsection{Anel de operadores diferenciais}

Já definimos a $n$-ésima álgebra de Weyl $A_{n}$ de duas maneiras: como uma $k$-subálgebra de $\operatorname{End}_{k}(k[X])$ gerada pelos operadores $x_{1}, \ldots, x_{n}$ e $\partial_{1}, \ldots, \partial_{n}$; e como um quociente de uma $k$-álgebra associativa livre em $2 n$ geradores $\left\{y_{1}, \ldots, y_{n}, z_{1}, \ldots, z_{n}\right\}$ por um ideal de relações.

Agora vamos definir de uma terceira maneira. Na verdade, vamos mostrar que as álgebras de Weyl são membros da família dos anéis de operadores diferenciais. Por esse motivo, a nésima álgebra de Weyl também é conhecida, como anel de operadores lineares diferenciais com coeficientes polinomiais.

Seja $R$ uma $k$-álgebra comutativa. Todo elemento $a \in R$ dá origem à um operador linear em $R$, a multiplicação à esquerda por $a$, isto é, $r \longmapsto a r$, para todo $r \in R$. O resultado disso, é a existência de um homomorfismo de anéis $R \longrightarrow \operatorname{End}_{k}(R)$, tal que $a \longmapsto \varphi_{a}$, em que $\varphi_{a} \in$ $\operatorname{End}_{k}(R)$ é a multiplicação à esquerda por $a$. Este homomorfismo é injetivo, pois se $\varphi_{a}=0$, então $\varphi_{a}(r)=a r=0$, para todo $r \in R$, em particular para $r=1_{R}$, isto é, $a 1_{R}=a=0$. Então podemos considerar $R$ como uma subálgebra de $\operatorname{End}_{k}(R)$. 
Definição 1.1.7. Uma $k$-derivação de $R$ é um operador linear $\delta \in \operatorname{End}_{k}(R)$ que satisfaz $\delta(a b)=\delta(a) b+a \delta(b)$, para todos $a, b \in R$ (Regra de Leibniz). O k-espaço vetorial de todas as $k$-derivações de $R$ é um $R$-módulo à esquerda denotado por $\operatorname{Der}_{k}(R)$, com a seguinte ação de $R:(a \delta)(b)=a \delta(b), \delta \in \operatorname{Der}_{k}(R), a, b \in R$. Além disso $\operatorname{Der}_{k}(R)$ também possui estrutura de álgebra de Lie sobre $k$ com o colchete definido pelo comutador, isto é, $\left[\delta_{i}, \delta_{j}\right]=\delta_{i} \delta_{j}-\delta_{j} \delta_{i}$, em que $\delta_{i}, \delta_{j} \in \operatorname{Der}_{k}(R)$.

Vamos definir, indutivamente, a ordem de um operador. Um operador linear $P \in \operatorname{End}_{k}(R)$ tem ordem zero se $[P, a]=0$, para todo $a \in R$. Suponhamos já definidos operadores com ordem $<n$. Um operador $P \in \operatorname{End}_{k}(R)$ tem ordem $n$ se ele não tem ordem menor do que $n$ e $[P, a]$ tem ordem menor do que $n$ para todo $a \in R$. Seja $D^{n}(R)$ o conjunto de todos os operadores lineares de $\operatorname{End}_{k}(R)$ com ordem $\leq n$.

$D^{n}(R)$ é um $k$-espaço vetorial. Vamos concluir usando indução em $n$. Se $P, Q \in D^{0}(R)$, temos que $[P+Q, a]=[P, a]+[Q, a]=0$, para todo $a \in R$, ou seja, $P+Q \in D^{0}(R)$. Vamos supor por indução que $P, Q \in D^{n-1}(R)$ implica $[P+Q, a] \in D^{n-2}(R)$, para todo $a \in R$, isto é, $P+Q \in D^{n-1}(R)$. Sejam $P, Q \in D^{n}(R)$, então $[P+Q, a]=[P, a]+[Q, a]$, para todo $a \in R$. Por hipótese de indução a soma em $D^{n-1}(R)$ é fechada e já que $P, Q \in D^{n}(R)$ é equivalente à $[P, a],[Q, a] \in D^{n-1}(R)$, para todo $a \in R$, temos que $[P, a]+[Q, a] \in D^{n-1}(R)$, ou seja, $[P+Q, a] \in D^{n-1}(R)$, para todo $a \in R$. Então $P+Q \in D^{n}(R)$. De forma análoga, mostra-se indutivamente que para $\lambda \in k$ e para $P \in D^{n}(R)$ temos que $\lambda P \in D^{n}(R)$. Como as operações soma e multiplicação por escalar são fechadas, $D^{n}(R) \subseteq \operatorname{End}_{k}(R)$, é um $k$-espaço vetorial.

Observação 1.1.2. Seja $R$ um anel. Sejam $a, b, c \in R$. Então a identidade de Jacobi é satisfeita, isto é, $[a,[b, c]]+[b,[c, a]]+[c,[a, b]]=0$.

Definição 1.1.8. Defina indutivamente $D^{0}(R)=\left\{P \in \operatorname{End}_{k}(R) \mid[P, a]=0, \forall a \in R\right\}$, e para $i>0, D^{i}(R)=\left\{P \in \operatorname{End}_{k}(R) \mid[P, a] \in D^{i-1}(R), \forall a \in R\right\}$. Claramente $D^{i}(R) \subseteq D^{j}(R)$ se $i<j$. Chamando $D(R)=\bigcup_{i=0}^{\infty} D^{i}(R)$, obtemos desta forma uma álgebra associativa, chamada de anel de operadores diferenciais de uma k-álgebra $R$.

Em outras palavras, o anel $D(R)$ é o conjunto de todos os operadores de $\operatorname{End}_{k}(R)$ com ordem finita, com as operações de soma e composição de operadores.

Para que esta definição faça sentido, devemos mostrar que a soma e o produto de dois operadores de ordem finita tem ordem finita. Antes, provaremos o seguinte resultado:

Lema 1.1.2. Os operadores de ordem $\leq 1$ correspondem aos elementos de $\operatorname{Der}_{k}(R)+R$. Os elementos de ordem zero são os elementos de $R$.

Demonstração. Seja $Q \in D^{1}(R)$. Como $Q \in \operatorname{End}_{k}(R)$ então $Q\left(1_{R}\right) \in R$. Tome $P=Q-$ $Q\left(1_{R}\right)$.

Observamos que $[a, b]=0$, para todos $a, b \in R$, pois $R$ é uma $k$-álgebra comutativa. Como cada elemento de $R$ dá origem à um operador linear em $R$, então todo $b \in R$ tem ordem zero, ou seja, $R \subseteq D^{0}(R)$. Assim $Q\left(1_{R}\right) \in D^{1}(R)$, e como esse é um $k$-espaço vetorial, $Q-Q\left(1_{R}\right) \in D^{1}(R)$, ou seja, $P \in D^{1}(R)$. Como $Q \in D^{1}(R),[Q, a] \in D^{0}(R)$, para todo $a \in$ $R$. Então $[b,[Q, a]]=0$, para todos $a, b \in R$. Usando a Observação 1.1 .2 e o fato $[b, a]=0$, para todos $a, b \in R$ :

$$
[b,[a, P]]+[a,[P, b]]+[P,[b, a]]=0 \Rightarrow[b,[a, P]]=[P, b] a-a[P, b]=[[Q, b], a]=0
$$

Portanto $P$ tem ordem $\leq 1$ e $[a, P]$ tem ordem zero para todo $a \in R$. Observamos também que $P\left(1_{R}\right)=\left(Q-Q\left(1_{R}\right)\right)\left(1_{R}\right)=Q\left(1_{R}\right)-Q\left(1_{R}\right)=0$. 
Como $[[P, a], b]=0$, para todo $b \in R$, escrevendo os comutadores explicitamente, obtemos a equação:

$$
(P a) b-(a P) b-b(P a)+b(a P)=0
$$

Aplicando este operador em $1_{R} \in R$, temos que $P(a b)=a P(b)+b P(a)-b a P\left(1_{R}\right)$. Como $P\left(1_{R}\right)=0$, segue que $P$ é uma $k$-derivação de $R$. Mas $Q=P+Q\left(1_{R}\right) \in \operatorname{Der}_{k}(R)+R$, como desejado.

Já sabemos que $R \subseteq D^{0}(R)$. Vamos mostrar que $D^{0}(R) \subseteq R$. Seja $Q \in D^{0}(R)$. Logo $[Q, a]=0$, para todo $a \in R$. Em particular $\left[Q, 1_{R}\right]=0$. Então $Q\left(1_{R}\right)-1_{R} Q=0$, isto é, $Q=Q\left(1_{R}\right) \in R$. Portanto $D^{0}(R)=R$.

Sendo assim, podemos caracterizar os operadores de ordem $\leq 1$.

Proposição 1.1.7. Sejam $P \in D^{n}(R)$ e $Q \in D^{m}(R)$. Então $P+Q$ tem ordem finita e $P Q$ $\in D^{n+m}(R)$.

Demonstração. Se $n=m$, trivialmente $P+Q \in D^{n}(R)$, ou seja, $P+Q$ tem ordem finita. Seja $n \neq m$. Podemos supor sem perda de generalidade que $n<m$. Como $D^{n}(R) \subseteq D^{m}(R)$, temos que $P, Q \in D^{m}(R)$, e como esse é $k$-espaço vetorial, $P+Q \in D^{m}(R)$. Então $P+Q$ tem ordem finita.

Agora vamos mostrar que $P Q \in D^{n+m}(R)$. Para isso, vamos usar indução em $m+n$. Se $m+n=0$, como $m, n \geq 0$, temos que $m=n=0$. Pelo Lema 1.1.2, $P, Q \in R$, $\operatorname{logo}$ $P Q \in R=D^{0}(R)$, e o resultado é válido. Suponhamos que o resultado é válido sempre que $m+n<k$. Se $m+n=k$ e $a \in R$, temos que:

$$
[P Q, a] \stackrel{\text { Obs.1.1.1 }}{=} P[Q, a]+[P, a] Q
$$

A definição de ordem implica que $[Q, a] \in D^{m-1}(R)$ e $[P, a] \in D^{n-1}(R)$. Então, pela hipótese de indução $P[Q, a],[P, a] Q \in D^{n+m-1}(R)$. $\operatorname{Logo}[P Q, a] \in D^{n+m-1}(R)$. Portanto $P Q \in$ $D^{n+m}(R)$, como desejado.

Agora vamos calcular explicitamente $\operatorname{Der}_{k}\left(k\left[x_{1}, \ldots, x_{n}\right]\right)$.

Proposição 1.1.8. Toda derivação de $k[X]=k\left[x_{1}, \ldots, x_{n}\right]$ é da forma $\sum_{i=1}^{n} f_{i} \partial_{i}$, para alguns $f_{1}, \ldots, f_{n} \in k[X]$.

Demonstração. É evidente que as derivadas parciais $\partial_{1}, \ldots, \partial_{n}$ são derivações de $k\left[x_{1}, \ldots, x_{n}\right]$. Seja $D \in \operatorname{Der}_{k}(k[X])$, uma derivação arbitrária.

Seja $x_{i}^{k} \in k[X]$. Vamos provar por indução em $k$ que $D\left(x_{i}^{k}\right)=k x_{i}^{k-1} D\left(x_{i}\right)$, para $i=$ $1, \ldots, n$. Se $k=1$, o resultado vale trivialmente. Suponhamos que $k>1$ e que o resultado seja válido para $k-1$. Então:

$$
D\left(x_{i}^{k}\right)=D\left(x_{i} x_{i}^{k-1}\right)=x_{i} D\left(x_{i}^{k-1}\right)+x_{i}^{k-1} D\left(x_{i}\right)
$$

Usando a hipótese de indução:

$D\left(x_{i}^{k}\right)=x_{i}(k-1) x_{i}^{k-2} D\left(x_{i}\right)+x_{i}^{k-1} D\left(x_{i}\right)=k x_{i}^{k-1} D\left(x_{i}\right)-x_{i}^{k-1} D\left(x_{i}\right)+x_{i}^{k-1} D\left(x_{i}\right)=k x_{i}^{k-1} D\left(x_{i}\right)$

Portanto $D\left(x_{i}^{k}\right)=k x_{i}^{k-1} D\left(x_{i}\right)$, para $i=1, \ldots, n$.

Vamos considerar um monômio qualquer de $k[X]$, isto é, $x_{1}^{s_{1}} \cdots x_{n}^{s_{n}}$, com $s_{1}, \ldots, s_{n} \in \mathbb{N}$.

Vamos calcular $\left(D-\sum_{i=1}^{n} D\left(x_{i}\right) \partial_{i}\right)\left(x_{1}^{s_{1}} \cdots x_{n}^{s_{n}}\right)$.

$$
D\left(x_{1}^{s_{1}} \cdots x_{n}^{s_{n}}\right)=\sum_{i=1}^{n} x_{1}^{s_{1}} \cdots \widehat{x_{i}^{s_{i}}} \cdots x_{n}^{s_{n}} D\left(x_{i}^{s_{i}}\right)=\sum_{i=1}^{n} s_{i} x_{1}^{s_{1}} \cdots x_{i}^{s_{i}-1} \cdots x_{n}^{s_{n}} D\left(x_{i}\right)
$$


$\mathrm{e}$

$$
\left(\sum_{i=1}^{n} D\left(x_{i}\right) \partial_{i}\right)\left(x_{1}^{s_{1}} \cdots x_{n}^{s_{n}}\right)=\sum_{i=1}^{n} D\left(x_{i}\right) \partial_{i}\left(x_{1}^{s_{1}} \cdots x_{n}^{s_{n}}\right)=\sum_{i=1}^{n} D\left(x_{i}\right) s_{i} x_{1}^{s_{1}} \cdots x_{i}^{s_{i}-1} \cdots x_{n}^{s_{n}}
$$

Portanto $\left(D-\sum_{i=1}^{n} D\left(x_{i}\right) \partial_{i}\right)\left(x_{1}^{s_{1}} \cdots x_{n}^{s_{n}}\right)=0$. Como os monômios $x_{1}^{s_{1}} \cdots x_{n}^{s_{n}}$, com $s_{1}, \ldots, s_{n}$ $\in \mathbb{N}$, formam uma base de $k[X]$, temos que $D-\sum_{i=1}^{n} D\left(x_{i}\right) \partial_{i}=0$, ou seja, $D=\sum_{i=1}^{n} D\left(x_{i}\right) \partial_{i}$, $\operatorname{com} D\left(x_{1}\right), \ldots, D\left(x_{n}\right) \in k[X]$.

Nosso objetivo é mostrar que a $n$-ésima álgebra de Weyl $A_{n}$ é o anel dos operadores diferenciais da $k$-álgebra dos polinômios em $n$ variáveis comutativas, ou seja, $D\left(k\left[x_{1}, \ldots, x_{n}\right]\right)=$ $D(k[X])=A_{n}$. Antes provaremos dois lemas.

Lema 1.1.3. Seja $P \in D(k[X])$. Se $\left[P, x_{i}\right]=0$, para todo $i=1, \ldots, n$, então $P \in k[X]$.

Demonstração. Vamos mostrar usando as hipóteses que $[P, f]=0$, para todo $f \in k[X]$. Como o comutador é bilinear, é necessário provar que $[P, f]=0$, quando $f$ é um monômio em $k[X]$.

Seja $f=x^{\alpha}$, para algum $\alpha \in \mathbb{N}^{n}$ e assuma que $\alpha_{i} \neq 0$. Vamos provar o resultado usando indução em $|\alpha|$. Se $|\alpha|=1$, por hipótese $\left[P, x_{i}\right]=0,1 \leq i \leq n$. Seja $|\alpha|>1$ e vamos supor que o resultado seja válido para todo $\beta \in \mathbb{N}^{n}$ tal que $|\beta|<|\alpha|$. Temos que:

$$
\left[P, x^{\alpha}\right]=\left[P, x_{i} x^{\alpha-e_{i}}\right] \stackrel{\text { Obs.1.1.1 }}{=}\left[P, x_{i}\right] x^{\alpha-e_{i}}+x_{i}\left[P, x^{\alpha-e_{i}}\right]
$$

Usando a hipótese de indução, isto é, $\left[P, x_{i}\right]=\left[P, x^{\alpha-e_{i}}\right]=0$, concluímos que $\left[P, x^{\alpha}\right]=0$. Portanto $[P, f]=0$, para todo $f \in k[X]$, ou seja, $P \in D^{0}(k[X])$.

Pelo Lema 1.1.2, concluímos que $P \in k[X]$.

Definição 1.1.9. Seja $C_{r}$ o conjunto dos operadores em $A_{n}$ que podem ser escritos na forma $\sum_{\alpha} f_{\alpha} \partial^{\alpha}$ com $|\alpha| \leq r$, em que $f_{\alpha} \in k[X]$.

Podemos pensar que $C_{r}$ é o conjunto dos operadores de $A_{n}$ com "ordem" $\leq r$ (veja Capítulo 2, Subseção 2.2.2). Sabemos que $D^{r}(k[X])$ é o conjunto dos operadores de $E_{n} d_{k}(k[X])$ com ordem $\leq r$. Logo $C_{r} \subseteq D^{r}(k[X])$. Se $P \in C_{r}$, então $P=\sum_{\alpha} f_{\alpha} \partial^{\alpha} \operatorname{com}|\alpha| \leq r, f_{\alpha} \in$ $k[X]$. Temos que $|\alpha| \leq r<r+1$, $\operatorname{logo} P \in C_{r+1}$. Então $C_{r} \subseteq C_{r+1} \cap D^{r}(k[X])$. Reciprocamente, se tomamos $Q \in C_{r+1} \cap D^{r}(k[X])$, ele terá ordem $\leq r$, por pertencer à $D^{r}(k[X])$. Logo $Q \in C_{r+1}$, será da forma $Q=\sum_{\beta} g_{\beta} \partial^{\beta} \operatorname{com}|\beta| \leq r, g_{\beta} \in k[X]$, ou seja, $Q \in C_{r}$. Então $C_{r+1} \cap D^{r}(k[X]) \subseteq C_{r}$.

Portanto

$$
C_{r}=C_{r+1} \cap D^{r}\left(k\left[x_{1}, \ldots, x_{n}\right]\right)
$$

Pela Proposição 1.1.8 temos que $C_{1}=\operatorname{Der}_{k}\left(k\left[x_{1}, \ldots, x_{n}\right]\right)+k\left[x_{1}, \ldots, x_{n}\right]$ e $C_{0}=k\left[x_{1}, \ldots, x_{n}\right]$.

O próximo lema é formalmente equivalente ao fato que todo campo vetorial $F=\left(F_{1}, \ldots, F_{n}\right)$ em $\mathbb{R}^{n}$ que satisfaz $\partial_{i} F_{j}=\partial_{j} F_{i}$, para todos $1 \leq i, j \leq n$, admite uma função potencial. No próximo lema usaremos a convenção de que se $k<n$ então $\mathbb{N}^{k}$ é identificado com o subconjunto de $\mathbb{N}^{n}$ com as últimas $n-k$ coordenadas nulas.

Lema 1.1.4. Sejam $P_{1}, \ldots, P_{n} \in C_{r-1}$ e suponha que $\left[P_{i}, x_{j}\right]=\left[P_{j}, x_{i}\right]$ para todos $1 \leq i, j \leq$ n. Então existe $Q \in C_{r}$ tal que $P_{i}=\left[Q, x_{i}\right]$, para $i=1, \ldots, n$.

Demonstração. Como $P_{n} \in C_{r-1}$, temos que $P_{n}=\sum_{\alpha} f_{\alpha} \partial^{\alpha}$, com $|\alpha| \leq r-1$ e $f_{\alpha} \in$ $k[X]$. Como $\alpha_{n} \in \mathbb{N}$, então $\left(\alpha_{n}+1\right) \neq 0$. Seja $\left(\alpha_{n}+1\right)^{-1}=1 /\left(\alpha_{n}+1\right)$. Podemos definir 
$Q^{\prime}=\sum_{\alpha}\left(\alpha_{n}+1\right)^{-1} f_{\alpha} \partial^{\alpha+e_{n}}, \operatorname{com}\left(\alpha_{n}+1\right)^{-1} f_{\alpha} \in k[X]$. Como $|\alpha| \leq r-1$, temos que $\left|\alpha+e_{n}\right|=|\alpha|+1 \leq r$. Portanto $Q^{\prime} \in C_{r}$. Vejamos agora que $\left[Q^{\prime}, x_{n}\right]=P_{n}$.

$$
\begin{aligned}
{\left[Q^{\prime}, x_{n}\right] } & =\left[\sum_{\alpha}\left(\alpha_{n}+1\right)^{-1} f_{\alpha} \partial^{\alpha+e_{n}}, x_{n}\right]=\sum_{\alpha}\left(\alpha_{n}+1\right)^{-1}\left[f_{\alpha} \partial^{\alpha+e_{n}}, x_{n}\right] \\
\stackrel{\text { Obs.1.1.1 }}{=} & \sum_{\alpha}\left(\alpha_{n}+1\right)^{-1}\left(f_{\alpha}\left[\partial^{\alpha+e_{n}}, x_{n}\right]+\left[f_{\alpha}, x_{n}\right] \partial^{\alpha+e_{n}}\right)=\sum_{\alpha}\left(\alpha_{n}+1\right)^{-1} f_{\alpha}\left[\partial^{\alpha} \partial_{n}, x_{n}\right] \\
\stackrel{\text { Obs.1.1.1 }}{=} & \sum_{\alpha}\left(\alpha_{n}+1\right)^{-1} f_{\alpha}\left(\partial^{\alpha}\left[\partial_{n}, x_{n}\right]+\left[\partial^{\alpha}, x_{n}\right] \partial_{n}\right) \stackrel{\text { Prop.1.1.4 }}{=} \sum_{\alpha}\left(\alpha_{n}+1\right)^{-1} f_{\alpha}\left(\alpha_{n}+1\right) \partial^{\alpha} \\
= & \sum_{\alpha} f_{\alpha} \partial^{\alpha}=P_{n}
\end{aligned}
$$

Suponhamos por indução que determinamos um $Q^{\prime} \in C_{r}$ tal que $\left[Q^{\prime}, x_{i}\right]=P_{i}$ para $k+1 \leq$ $i \leq n$. Desta maneira, pelas hipóteses, $\left[\left[Q^{\prime}, x_{i}\right], x_{k}\right]=\left[P_{k}, x_{i}\right]$, para $k+1 \leq i \leq n$. Vamos provar que o resultado é válido para $k$.

Seja $G=\left[Q^{\prime}, x_{k}\right]-P_{k} \in A_{n}$. Então $\left[G, x_{i}\right]=0$, para $k+1 \leq i \leq n$. De fato:

$$
\begin{aligned}
{\left[G, x_{i}\right] } & =\left[\left[Q^{\prime}, x_{k}\right], x_{i}\right]-\left[P_{k}, x_{i}\right]=\left[\left[Q^{\prime}, x_{k}\right], x_{i}\right]-\left[P_{i}, x_{k}\right] \\
& =\left[\left[Q^{\prime}, x_{k}\right], x_{i}\right]-\left[\left[Q^{\prime}, x_{i}\right], x_{k}\right] \stackrel{\text { Obs.1.1.2 }}{=}\left[\left[x_{i}, x_{k}\right], Q^{\prime}\right]=\left[0, Q^{\prime}\right]=0
\end{aligned}
$$

Segue da identidade $\left[\partial^{\beta}, x_{n}\right]=\beta_{n} \partial^{\beta-e_{n}}$ (Proposição 1.1.4), que se $\left[\partial^{\beta}, x_{n}\right]=0$, então $\beta_{n}=$ 0 . Então $\left[G, x_{n}\right]=0$ implica, pela Proposição 1.1.2, que $G$ pode ser escrito como uma combinação linear de monômios da forma $x^{\alpha} \partial^{\beta} \operatorname{com} \beta \in \mathbb{N}^{n-1}$. Como $\left[G, x_{i}\right]=0$, para $k+1 \leq i \leq n$, podemos aplicar esse resultado várias vezes e concluir que $G=\sum_{\alpha \in \mathbb{N} k} f_{\alpha} \partial^{\alpha}$ $\operatorname{com} f_{\alpha} \in k[X]$. Seja $Q^{\prime \prime}=\sum_{\alpha \in \mathbb{N}^{k}}\left(\alpha_{k}+1\right)^{-1} f_{\alpha} \partial^{\alpha+e_{k}}$.

$Q^{\prime} \in C_{r} \subseteq D^{r}(k[X])$ implica que $\left[Q^{\prime}, x_{k}\right] \in C_{r} \cap D^{r-1}(k[X])=C_{r-1}$. Como $P_{k} \in C_{r-1}, G$ também pertence à $C_{r-1}$. Logo $|\alpha| \leq r-1$. Então $\left|\alpha+e_{k}\right|=|\alpha|+1 \leq r$, ou seja, $Q^{\prime \prime} \in C_{r}$. Por construção $\left[Q^{\prime \prime}, x_{i}\right]=0$, para $k+1 \leq i \leq n$. Assim $\left[Q^{\prime}-Q^{\prime \prime}, x_{i}\right]=\left[Q^{\prime}, x_{i}\right]-\left[Q^{\prime \prime}, x_{i}\right]=P_{i}$, para $k+1 \leq i \leq n$. Mas $\left[Q^{\prime \prime}, x_{k}\right]=G$. De fato:

$$
\left[Q^{\prime \prime}, x_{k}\right]=\sum_{\alpha \in \mathbb{N}^{k}}\left(\alpha_{k}+1\right)^{-1} f_{\alpha}\left[\partial^{\alpha} \partial_{k}, x_{k}\right]=\sum_{\alpha \in \mathbb{N}^{k}}\left(\alpha_{k}+1\right)^{-1} f_{\alpha}\left(\alpha_{k}+1\right) \partial^{\alpha}=G
$$

Então $\left[Q^{\prime}-Q^{\prime \prime}, x_{k}\right]=\left[Q^{\prime}, x_{k}\right]-\left[Q^{\prime \prime}, x_{k}\right]=\left[Q^{\prime}, x_{k}\right]-G=P_{k}$. Portanto $\left[Q^{\prime}-Q^{\prime \prime}, x_{i}\right]=P_{i}$, para $k \leq i \leq n$, e a indução está completa.

Teorema 1.1.5. O anel dos operadores diferenciais de $k\left[x_{1}, \ldots, x_{n}\right]$ é $A_{n}$, ou seja, $D\left(k\left[x_{1}, \ldots, x_{n}\right]\right)=A_{n}$. Além disso, $D^{m}\left(k\left[x_{1}, \ldots, x_{n}\right]\right)=C_{m}$.

Demonstração. É suficiente provar que $D^{m}\left(k\left[x_{1}, \ldots, x_{n}\right]\right) \subseteq C_{m}$, pois a inclusão oposta é clara. Faremos indução em $m$. Seja $P \in D\left(k\left[x_{1}, \ldots, x_{n}\right]\right)$. Se $m=0$, claramente $C_{0}=$ $D^{0}\left(k\left[x_{1}, \ldots, x_{n}\right]\right)$. Se $P \in D^{1}\left(k\left[x_{1}, \ldots, x_{n}\right]\right)$, então pelo Lema 1.1.2, $P \in \operatorname{Der}_{k}\left(k\left[x_{1}, \ldots, x_{n}\right]\right)+$ $k\left[x_{1}, \ldots, x_{n}\right]$. Assim $P \in C_{1}$, pela Proposição 1.1.8. Suponha, por indução, que $D^{r}\left(k\left[x_{1}, \ldots, x_{n}\right]\right)=C_{r}$ para $r \leq m-1$. Seja $P \in D^{m}\left(k\left[x_{1}, \ldots, x_{n}\right]\right)$. Para $1 \leq i \leq n$, tome $P_{i}=\left[P, x_{i}\right]$. Como $P \in D^{m}\left(k\left[x_{1}, \ldots, x_{n}\right]\right), P_{i}$ tem ordem $r \leq m-1$, e segue que $P_{i} \in$ $C_{m-1}$. Mas, para todos $1 \leq i, j \leq n$,

$$
\left[P_{i}, x_{j}\right]=\left[\left[P, x_{i}\right], x_{j}\right] \stackrel{\text { Obs.1.1.2 }}{=}\left[\left[P, x_{j}\right], x_{i}\right]=\left[P_{j}, x_{i}\right]
$$

Então pelo Lema 1.1.4 existe $Q \in C_{m}$ tal que $\left[Q, x_{i}\right]=P_{i}, 1 \leq i \leq n$. Assim $\left[Q-P, x_{i}\right]=0$ em $D\left(k\left[x_{1}, \ldots, x_{n}\right]\right)$. Uma vez que é válido para $1 \leq i \leq n$, concluímos pelo Lema 1.1.3 que 
$Q-P \in k\left[x_{1}, \ldots, x_{n}\right]=C_{0} \subseteq C_{m}$. Portanto como $Q \in C_{m}$ e $Q-P \in C_{m}$, temos que $P \in$ $C_{m}$. Assim $D^{m}\left(k\left[x_{1}, \ldots, x_{n}\right]\right) \subseteq C_{m}$, ou seja, $D^{m}\left(k\left[x_{1}, \ldots, x_{n}\right]\right)=C_{m}$, como desejado.

Se $P \in D\left(k\left[x_{1}, \ldots, x_{n}\right]\right)$, existe $r$ tal que $P \in D^{r}\left(k\left[x_{1}, \ldots, x_{n}\right]\right)=C_{r} \subseteq A_{n}$, ou seja, $D\left(k\left[x_{1}, \ldots, x_{n}\right]\right) \subseteq A_{n}$. Reciprocamente, se $Q \in A_{n}, Q=\sum_{\alpha} f_{\alpha} \partial^{\alpha}$, com $f_{\alpha} \in k\left[x_{1}, \ldots, x_{n}\right]$. Logo, existe $m$ tal que $|\alpha| \leq m$, para todo $\alpha$ que aparece como expoente em $\partial$. Logo $Q \in$ $C_{m}=D^{m}\left(k\left[x_{1}, \ldots, x_{n}\right]\right)$. Assim $A_{n} \subseteq D\left(k\left[x_{1}, \ldots, x_{n}\right]\right)$. Portanto:

$$
D\left(k\left[x_{1}, \ldots, x_{n}\right]\right)=A_{n}
$$

\subsection{Problemas com corpos com característica prima}

Como foi observado no começo deste Capítulo, é essencial que o corpo base sobre o qual a álgebra de Weyl está definida tenha característica zero. Entretanto, não é imediatamente claro porque se deve fazer tal restrição. Afinal, a definição da álgebra de Weyl pode fazer todo sentido sem qualquer restrição na característica do corpo $k$.

O problema é mais profundo. Em primeiro lugar, a álgebra de Weyl com característica prima sofre com problema de dupla personalidade. Isto acontece porque os dois caminhos pelos quais nós definimos $A_{n}$ (como um anel de operadores diferenciais e como o quociente de uma $k$-álgebra livre por um ideal de relações) serão dois anéis não isomorfos. Vejamos o que acontece para o corpo $\mathbb{Z}_{p}$, em que $p$ é primo, no caso de uma variável.

Considere primeiro a álgebra de operadores $R_{1}$ gerada por $\mathbb{Z}_{p}[x]$ e sua derivação $\partial \mathrm{em}$ relação à variàvel $x$. Vamos calcular $\partial^{p}\left(x^{k}\right)$. Se $k<p$, então $\partial^{p}\left(x^{k}\right)=0$. Se $k \geq p$, então:

$$
\partial^{p}\left(x^{k}\right)=k \ldots(k-p+1) x^{k-p}=0
$$

pois o coeficiente é divisível por $p$. Então $\partial^{p}=0$ como operador em $\mathbb{Z}_{p}[x]$. Concluímos que o anel de operadores $R_{1}$ tem elementos nilpotentes. Seja $b \in R_{1}$, um elemento nilpotente. Então existe $n \in \mathbb{N}$ tal que $b^{n}=0$ e $b^{n-1} \neq 0$. Sendo assim $0=b^{n}=b b^{n-1}$. Portanto, em particular, $R_{1}$ não é um domínio.

Agora considere o anel $R_{2}$ gerado sobre $\mathbb{Z}_{p}$ por $y, z$ sujeito à relação $[z, y]=1$. Este anel é um domínio. Logo $R_{1}$ e $R_{2}$ são anéis não isomorfos. Contudo, $R_{2}$ não é igual à álgebra de Weyl com característica zero em outro aspecto: não é um anel simples.

Por exemplo, seja $f \in \mathbb{Z}_{p}[y]$ então $[z, f]=\partial f / \partial y$. Em particular, $\left[z, y^{p}\right]=p y^{p-1}=0$, sobre $\mathbb{Z}_{p}$. Segue que $y^{p}$ comuta com todo elemento de $R_{2}$. Então o ideal gerado por $y^{p}$ em $R_{2}$ é um ideal bilateral. Em particular, $R_{2}$ não é um anel simples.

Estes comentários superficiais servem para mostrar que o caso da característica prima é muito diferente do caso da característica zero. A álgebra de Weyl com característica prima é discutida com maiores detalhes em [Smi85].

\section{3 Álgebra de Weyl de posto infinito}

Agora vamos definir a álgebra de Weyl de posto infinito. Como já mencionamos, o corpo $k$ tem característica zero. Vamos exigir nesta seção que ele também seja algebricamente fechado. Vamos basear essa seção em [FGM14][Seções 2.1 e 2.2].

Seja I um conjunto infinito enumerável satisfazendo a seguinte condição: $|\mathrm{I}|<|k|$ (a cardinalidade do conjunto I é menor que a cardinalidade do corpo $k$ ). Seja $B$ a $k$-álgebra comutativa dos polinômios em infinitas variáveis $x_{i}, i \in \mathrm{I}$. 
Vamos considerar o grupo abeliano aditivo $k^{\mathrm{I}}$ consistindo de todos os vetores $\mathbf{v}=\left(v_{i}\right)_{i \in \mathrm{I}}$ com coeficientes em $k$. Seja $\mathbb{Z}_{f}^{\mathrm{I}}$ o subgrupo de $k^{\mathrm{I}}$, formado por todos os vetores com coeficientes inteiros, com no máximo, uma quantidade finita de coeficientes não nulos. Para $\mathbf{v}$ $\in \mathbb{Z}_{f}^{\mathrm{I}}$ denotaremos por $\mathbf{x}^{\mathbf{v}}$ o monômio $\prod_{i \in \mathrm{I}} x_{i}^{v_{i}}$. Denotaremos por $\mathbb{N}_{f}^{\mathrm{I}}$ o conjunto de todos os vetores pertencentes a $\mathbb{Z}_{f}^{\mathrm{I}}$ em que todos os coeficientes são inteiros não negativos.

O anel de polinômios $k[x]$ com uma variável $x$ é um $k$-espaço vetorial, que tem uma base infinita formada por $1, x, x^{2}, \ldots$. No caso do anel de polinômios com várias variáveis $x_{1}, \ldots, x_{n}$, um monômio é um produto $x_{1}^{\alpha_{1}} \cdots x_{n}^{\alpha_{n}}$, em que $\alpha_{1}, \ldots, \alpha_{n}$ são inteiros não negativos. Similar ao caso com uma variável, os polinômios com variáveis $x_{1}, \ldots, x_{n}$, formam um $k$-espaço vetorial que tem uma base formada por todos os monômios chamada de base monomial ou base canônica.

Portanto, os monômios $\left\{\mathbf{x}^{\mathbf{v}} \mid \mathbf{v} \in \mathbb{N}_{f}^{\mathrm{I}}\right\}$ formam uma base para $B$ como $k$-espaço vetorial. De fato, se $p\left(x_{i_{1}}, \ldots, x_{i_{n}}\right) \in B$, é um polinômio nas variáveis $x_{i_{1}}, \ldots, x_{i_{n}}$, com $i_{1}, \ldots, i_{n} \in \mathrm{I}$, podemos considerar que $p\left(x_{i_{1}}, \ldots, x_{i_{n}}\right) \in k\left[x_{i_{1}}, \ldots, x_{i_{n}}\right]$. Podemos definir a álgebra dos polinômios $k\left[x_{i_{1}}, \ldots, x_{i_{n}}\right]$ com $n$ variáveis comutativas $x_{i_{1}}, \ldots, x_{i_{n}}$ por iteração $k\left[x_{i_{1}}, \ldots, x_{i_{n}}\right]=$ $k\left[x_{i_{1}}, \ldots, x_{i_{n-1}}\right]\left[x_{i_{n}}\right]$. Esta definição corresponde à ideia de que uma soma de monômios em $n$ variáveis comutativas $x_{i_{1}}, \ldots, x_{i_{n}}$ deve ser escrita como um polinômio na última variável $x_{i_{n}}$, colocando-a em evidência em cada monômio, em que os coeficientes pertencem à $k\left[x_{i_{1}}, \ldots, x_{i_{n-1}}\right]$ (para mais detalhes indicamos [SZ91], Capítulo $I$ ). Dessa forma existem: um inteiro não negativo $m$, coeficientes $c_{\alpha_{i_{1}} \ldots \alpha_{i_{n}}} \in k$ e $0 \leq \alpha_{i_{1}}, \ldots, \alpha_{i_{n}} \leq m$ inteiros não negativos tais que:

$$
p\left(x_{i_{1}}, \ldots, x_{i_{n}}\right)=\sum_{\alpha_{i_{1}}, \ldots, \alpha_{i_{n}}=0}^{m} c_{\alpha_{i_{1}} \ldots \alpha_{i_{n}}} x_{i_{1}}^{\alpha_{i_{1}}} \cdots x_{i_{n}}^{\alpha_{i_{n}}}
$$

ou seja, $p\left(x_{i_{1}}, \ldots, x_{i_{n}}\right)$ é uma combinação linear de monômios com coeficientes em $k$. Os coeficientes $c_{\alpha_{i_{1}} \ldots \alpha_{i_{n}}}$ na representação canônica acima são únicos, pois se considerarmos duas possíveis representações canônicas do mesmo polinômio $p\left(x_{i_{1}}, \ldots, x_{i_{n}}\right)$, isto é,

$$
p\left(x_{i_{1}}, \ldots, x_{i_{n}}\right)=\sum_{\alpha_{i_{1}}, \ldots, \alpha_{i_{n}}=0}^{m} c_{\alpha_{i_{1}} \ldots \alpha_{i_{n}}} x_{i_{1}}^{\alpha_{i_{1}}} \cdots x_{i_{n}}^{\alpha_{i_{n}}}=\sum_{\alpha_{i_{1}}, \ldots, \alpha_{i_{n}}=0}^{m} d_{\alpha_{i_{1}} \ldots \alpha_{i_{n}}} x_{i_{1}}^{\alpha_{i_{1}}} \cdots x_{i_{n}}^{\alpha_{i_{n}}}
$$

usando indução em $n$, concluímos que $c_{\alpha_{i_{1}} \ldots \alpha_{i_{n}}}=d_{\alpha_{i_{1}} \ldots \alpha_{i_{n}}}$, para todos $0 \leq \alpha_{i_{1}}, \ldots, \alpha_{i_{n}} \leq m$.

Definição 1.3.1 (Álgebra de Weyl de posto infinito). Para $i \in \mathrm{I}$, seja $X_{i}=x_{i}$ o operador linear em $B$ dado pela multiplicação por $x_{i}$. Seja $Y_{i}=\partial_{i}$, o operador linear em $B$ dado pela derivada parcial com respeito à variável $x_{i}$, isto é, $\partial_{i}$ é uma derivação de $B$ definida por $\partial_{i} \cdot x_{j}=\delta_{i j}$ nos geradores, em que $\delta_{i j}$ é o simbolo do delta de Kronecker. A álgebra de Weyl de posto infinito $A=A_{k, \mathrm{I}}$ é a subálgebra de $\operatorname{End}_{k}(B)$ gerada por todos $X_{i}$ e $Y_{i}, i \in \mathrm{I}$.

Quando dizemos que o posto de $A$ é infinito, queremos dizer que ela é gerada por uma quantidade infinita de elementos.

É fácil de verificar que os geradores satisfazem as seguintes relações:

$$
\begin{gathered}
{\left[Y_{i}, X_{j}\right]=\delta_{i j} \cdot 1} \\
{\left[X_{i}, X_{j}\right]=\left[Y_{i}, Y_{j}\right]=0}
\end{gathered}
$$

para todo $i, j \in \mathrm{I}$, em que 1 é o operador identidade e 0 o operador nulo. 
Estas relações dão uma apresentação para a álgebra $A$, ou seja, qualquer outra relação entre os geradores de $A$, é combinação destas relações listadas acima.

Definição 1.3.2. Para $i \in \mathrm{I}$, seja $t_{i}=X_{i} Y_{i}=x_{i} \partial_{i} \in A$. Denotaremos por $A_{0}$ a subálgebra de A gerada por todos $t_{i}, i \in \mathrm{I}$.

Proposição 1.3.1. $A_{0}$ é uma subálgebra comutativa de $A$.

Demonstração. Sabemos que $\left[Y_{i}, X_{j}\right]=\delta_{i j} \cdot 1,\left[X_{i}, X_{j}\right]=0$ e $\left[Y_{i}, Y_{j}\right]=0$ para todo $i, j \in \mathrm{I}$. Vamos verificar que quaisquer dois geradores de $A_{0}$ comutam. Sejam $t_{i}, t_{j} \in A_{0}$, com $i \neq j$. Então:

$$
t_{i} t_{j}=X_{i} Y_{i} X_{j} Y_{j}=X_{i} X_{j} Y_{i} Y_{j}=X_{j} X_{i} Y_{j} Y_{i}=X_{j} Y_{j} X_{i} Y_{i}=t_{j} t_{i}
$$

Para $\mathbf{v} \in \mathbb{Z}_{f}^{\mathrm{I}}$ definimos o elemento:

$$
\mathbf{X}_{\mathbf{v}}=\prod_{i: v_{i}>0} X_{i}^{v_{i}} \prod_{i: v_{i}<0} Y_{i}^{-v_{i}}
$$

Proposição 1.3.2. A álgebra de Weyl de posto infinito A é livre tanto como $A_{0}$-módulo à esquerda como à direita, com base $\left\{\mathbf{X}_{\mathbf{v}} \mid \mathbf{v} \in \mathbb{Z}_{f}^{\mathrm{I}}\right\}$.

Demonstração. Vamos provar que $A$ é livre como $A_{0}$-módulo à esquerda. De maneira análoga é possível provar que também é livre como $A_{0}$-módulo à direita.

Considere um monômio nos geradores de $A$, isto é, $Z_{k_{1}} \cdots Z_{k_{r}}$, com $Z_{k_{l}} \in\left\{X_{i}, Y_{j} \mid i, j \in\right.$ I $\}, 1 \leq l \leq r$. Usando a relação $\left[Y_{j}, X_{i}\right]=\delta_{j i} \cdot 1$, podemos "reorganizar" o monômio de maneira que ele seja uma soma de monômios em que todos os $X$ 's estão à esquerda de todos os $Y$ 's, com coeficientes em $k$, ou seja, $Z_{k_{1}} \cdots Z_{k_{r}}=\sum a_{i_{1} \cdots i_{m} j_{1} \cdots j_{n}} X_{i_{1}}^{u_{i_{1}}} \cdots X_{i_{m}}^{u_{i_{m}}} Y_{j_{1}}^{v_{j_{1}}} \cdots Y_{j_{n}}^{v_{j_{n}}}$, em que $a_{i_{1} \cdots i_{m} j_{1} \cdots j_{n}} \in k,\left\{i_{1}, \cdots, i_{m}, j_{1}, \cdots, j_{n}\right\} \in \mathrm{I},\left(u_{i_{1}}, \ldots, u_{i_{m}}\right) \in \mathbb{N}^{m}$ e $\left(v_{j_{1}}, \ldots, v_{j_{n}}\right) \in \mathbb{N}^{n}$ . Podemos reescrever da seguinte maneira:

$$
Z_{k_{1}} \cdots Z_{k_{r}}=\sum a_{i_{1} \cdots i_{m} j_{1} \cdots j_{n}} X_{i_{1}}^{u_{i_{1}}} \cdots X_{i_{m}}^{u_{i_{m}}} Y_{j_{1}}^{-\left(-v_{j_{1}}\right)} \cdots Y_{j_{n}}^{-\left(-v_{j_{n}}\right)}
$$

em que $-v_{j_{1}}<0, \ldots,-v_{j_{n}}<0$. Como $k \subset A_{0}$, temos que $A$ é gerado pelo conjunto $\left\{\mathbf{X}_{\mathbf{v}} \mid \mathbf{v} \in\right.$ $\left.\mathbb{Z}_{f}^{\mathrm{I}}\right\}$ como $A_{0}$-módulo à esquerda.

Agora vamos provar que o conjunto é linearmente independente sobre $A_{0}$. Sabemos que $Y_{j}, j \in \mathrm{I}$, é o operador linear em $B$ dado pela derivada parcial com respeito à variável $x_{j}$, ou seja, $Y_{j}\left(x_{i}\right)=\partial_{j}\left(x_{i}\right)=\delta_{j i} \cdot 1$. Dado $\mathbf{v}=\left(v_{i}\right)_{i \in \mathrm{I}} \in \mathbb{Z}_{f}^{\mathrm{I}}$, se $v_{i}<0$, então $v_{i}$ será expoente de $Y_{i}$ com sinal oposto, ou seja, $Y_{i}^{-v_{i}}$. Dessa forma $-v_{i}$ é um inteiro não negativo. Considerando $Y_{j_{1}}^{-v_{j_{1}}} \ldots Y_{j_{n}}^{-v_{j_{n}}}$, com $\left\{j_{1}, \ldots, j_{n}\right\} \in$ I, temos que $\left(-v_{j_{1}}, \ldots,-v_{j_{n}}\right) \in \mathbb{N}^{n}$. Seja $P=\sum_{u, v} a_{u v} X_{i_{1}}^{u_{i_{1}}} \cdots X_{i_{m}}^{u_{i_{m}}} Y_{j_{1}}^{-v_{j_{1}}} \cdots Y_{j_{n}}^{-v_{j_{n}}}$, com $a_{u v} \in A_{0}$, em que $u=\left(u_{i_{1}}, \ldots, u_{i_{m}}\right) \in \mathbb{N}^{m} \mathrm{e}$ $v=\left(-v_{j_{1}}, \ldots,-v_{j_{n}}\right) \in \mathbb{N}^{n}$. Vamos provar que se algum $a_{u v} \neq 0$ então $P=\sum_{u, v} a_{u v} X^{u} Y^{v} \neq$ 0 . Seja $w=\left(-w_{j_{1}}, \ldots,-w_{j_{n}}\right) \in \mathbb{N}^{n}$ o menor multi-índice, com respeito à ordem lexicográfica, que aparece como expoente de $Y$ em $P$. Notamos que se $r<n$, então $\mathbb{N}^{r}$ é identificado com o subconjunto de $\mathbb{N}^{n}$ em que as últimas $n-r$ coordenadas são nulas, ou seja, se $w \in$ $\mathbb{N}^{r}$ então $w \in \mathbb{N}^{n}$, basta "completar" as $n-r$ coordenadas com zeros. Vamos considerar $x^{w}=x_{j_{1}}^{-w_{j_{1}}} \cdots x_{j_{n}}^{-w_{j_{n}}} \in B$. Observamos que $-w_{j_{1}}>0, \ldots,-w_{j_{n}}>0$. Usando o Lema 1.1.1, temos que $P\left(x^{w}\right)=\sum_{u, v} a_{u v} X^{u} Y^{v}\left(x^{w}\right)=w ! \sum_{u} a_{u w} X^{u}$, pois se $w$ é estritamente menor que $v$, na ordem lexicográfica, então $Y^{v}\left(x^{w}\right)=0$. Pela escolha de $w$, existe $u \in \mathbb{N}^{m}$ tal que $a_{u w} \neq 0$. Como $A$ não possui divisores de zero implica que $a_{u w} X^{u} \neq 0$. Temos que $P\left(x^{w}\right)$ é não nulo, pois no mínimo um dos coeficientes $a_{u w}$ é não nulo. Portanto $P \neq 0$, como desejado. 
Proposição 1.3.3. Os geradores de $A_{0}, t_{i}, i \in \mathrm{I}$, são algebricamente independentes sobre $k$.

Demonstração. Seja $R$ uma $k$-álgebra. Dizemos que os elementos $u_{1}, \ldots, u_{n} \in R$ são algebricamente independentes sobre $k$ se não existe um polinômio não nulo $f\left(x_{1}, \ldots, x_{n}\right)$ no anel de polinômios $k\left[x_{1}, \ldots, x_{n}\right]$ tal que $f\left(u_{1}, \ldots, u_{n}\right)=0$.

Queremos provar que o conjunto $\left\{t_{i}\right\}_{i \in \mathrm{I}}$ é algebricamente independente sobre $k$. Basta provar para uma quantidade finita de elementos. Seja $\left\{t_{i_{1}}, \ldots, t_{i_{n}}\right\}$, em que $i_{1}, \ldots, i_{n} \in \mathrm{I}$, e $i_{k} \neq i_{l}, 1 \leq k, l \leq n$.

Seja $p\left(x_{i_{1}}, \ldots, x_{i_{n}}\right) \in B$, um polinômio não nulo, nas variáveis $x_{i_{1}}, \ldots, x_{i_{n}}, \operatorname{com} i_{1}, \ldots, i_{n}$ $\in$ I. Então $p\left(x_{i_{1}}, \ldots, x_{i_{n}}\right)=\sum_{\alpha_{i_{1}}, \ldots, \alpha_{i_{n}}} c_{\alpha_{i_{1}} \ldots \alpha_{i_{n}}} x_{i_{1}}^{\alpha_{i_{1}}} \cdots x_{i_{n}}^{\alpha_{i_{n}}}, \operatorname{com} c_{\alpha_{i_{1}} \ldots \alpha_{i_{n}}} \in k$ e $\alpha_{i_{1}}, \ldots, \alpha_{i_{n}}$ são inteiros não negativos.

Vamos supor que $p\left(t_{i_{1}}, \ldots, t_{i_{n}}\right)=0$, ou seja:

$$
\sum_{\alpha_{i_{1}}, \ldots, \alpha_{i_{n}}} c_{\alpha_{i_{1}} \ldots \alpha_{i_{n}}} t_{i_{1}}^{\alpha_{i_{1}}} \cdots t_{i_{n}}^{\alpha_{i_{n}}}=0 \Longrightarrow \sum_{\alpha_{i_{1}}, \ldots, \alpha_{i_{n}}} c_{\alpha_{i_{1}} \ldots \alpha_{i_{n}}}\left(X_{i_{1}} Y_{i_{1}}\right)^{\alpha_{i_{1}}} \cdots\left(X_{i_{n}} Y_{i_{n}}\right)^{\alpha_{i_{n}}}=0
$$

Seja $\left(X_{j} Y_{j}\right)^{\alpha_{j}}, j \in \mathrm{I}, \alpha_{j}$ um inteiro não negativo. Temos que $\underbrace{\left(X_{j} Y_{j}\right) \cdots\left(X_{j} Y_{j}\right)}_{\alpha_{j} \text { vezes }}$. Usando a relação $\left[Y_{j}, X_{j}\right]=1,\left(X_{j} Y_{j}\right)^{\alpha_{j}}=\sum_{\beta_{j}} d_{\beta_{j}} X_{j}^{\beta_{j}} Y_{j}^{\beta_{j}}$, com $d_{\beta_{j}} \in k$ e $\beta_{j}$ é um inteiro não negativo. Então:

$$
\sum_{\beta_{i_{1}}, \ldots, \beta_{i_{n}}} d_{\beta_{i_{1}} \ldots \beta_{i_{n}}} X_{i_{1}}^{\beta_{i_{1}}} Y_{i_{1}}^{\beta_{i_{1}}} \cdots X_{i_{n}}^{\beta_{i_{n}}} Y_{i_{n}}^{\beta_{i_{n}}}=0
$$

$\operatorname{com} d_{\beta_{i_{1}} \ldots \beta_{i_{n}}} \in k$ e $\beta_{i_{1}}, \ldots, \beta_{i_{n}}$ são inteiros não negativos.

Como $i_{k} \neq i_{l}, 1 \leq k, l \leq n$, temos que $\sum_{\beta_{i_{1}}, \ldots, \beta_{i_{n}}} d_{\beta_{i_{1}} \ldots \beta_{i_{n}}} X_{i_{1}}^{\beta_{i_{1}}} \cdots X_{i_{n}}^{\beta_{i_{n}}} Y_{i_{1}}^{\beta_{i_{1}}} \cdots Y_{i_{n}}^{\beta_{i_{n}}}=0$. Seja $\sigma=\left(\sigma_{i_{1}}, \ldots, \sigma_{i_{n}}\right)$ o menor multi-índice, com respeito à ordem lexicográfica, que aparece como expoente de $Y$, ou seja, $Y_{i_{1}}^{\sigma_{i_{1}}} \cdots Y_{i_{n}}^{\sigma_{i_{n}}}$. Observamos que $d_{\sigma_{i_{1}} \ldots \sigma_{i_{n}}} \neq 0$ e $X_{i_{1}}^{\sigma_{i_{1}}} \cdots X_{i_{n}}^{\sigma_{i_{n}}} \neq 0$.

Escolhemos $x_{i_{1}}^{\sigma_{i_{1}}} \cdots x_{i_{n}}^{\sigma_{i_{n}}}=x^{\sigma} \in B$. Como $p\left(t_{i_{1}}, \ldots, t_{i_{n}}\right)=0$ é o operador nulo em $B$, temos que $p\left(t_{i_{1}}, \ldots, t_{i_{n}}\right)\left(x^{\sigma}\right)=0$, isto é, $\sum_{\beta_{i_{1}}, \ldots, \beta_{i_{n}}} d_{\beta_{i_{1}} \ldots \beta_{i_{n}}} X_{i_{1}}^{\beta_{i_{1}}} \cdots X_{i_{n}}^{\beta_{i_{n}}} Y_{i_{1}}^{\beta_{i_{1}}} \cdots Y_{i_{n}}^{\beta_{i_{n}}}\left(x^{\sigma}\right)=0$.

Pelo Lema 1.1.1 temos que $0=p\left(t_{i_{1}}, \ldots, t_{i_{n}}\right)\left(x^{\sigma}\right)=\sigma !\left(d_{\sigma_{i_{1}} \ldots \sigma_{i_{n}}} X_{i_{1}}^{\sigma_{i_{1}}} \cdots X_{i_{n}}^{\sigma_{i_{n}}}\right)$. Isso é um absurdo, pois a álgebra $A$ não possui divisores de zero. Então, não existe polinômio não nulo em $B$ que anule $\left\{t_{i_{1}}, \ldots, t_{i_{n}}\right\}$. Portanto $\left\{t_{i}\right\}_{i \in \mathrm{I}}$ é algebricamente independente sobre $k$.

Proposição 1.3.4. Nenhum elemento $\mathbf{X}_{\mathbf{v}}, \operatorname{com} \mathbf{v} \neq 0, \mathbf{v} \in \mathbb{Z}_{f}^{\mathrm{I}}$, comuta com todos os elementos de $A_{0}$.

Demonstração. Seja $Z$ uma combinação linear de elementos da forma $\prod_{i: u_{i}>0} X_{i}^{u_{i}} \prod_{i: u_{i}<0} Y_{i}^{-u_{i}}$, em que $\mathbf{u} \in \mathbb{Z}_{f}^{\mathrm{I}}$ e os coeficientes estão em $k$, ou seja, $Z=\sum_{\mathbf{u}} a_{\mathbf{u}} \mathbf{X}_{\mathbf{u}}$, com $a_{\mathbf{u}} \in k$. Usando a ordem lexicográfica podemos considerar $Z=a_{\mathbf{v}} \mathbf{X}_{\mathbf{v}}+Z^{\prime}$, com $\mathbf{v} \neq 0$, em que $\operatorname{deg}\left(Z^{\prime}\right)<$ $\operatorname{deg}\left(\mathbf{X}_{\mathbf{v}}\right)$.

Suponhamos que $Z A_{0}=A_{0} Z$, isto é, $Z a=a Z$, para todo $a \in A_{0}$.

Temos que $\mathbf{X}_{\mathbf{v}}=X_{i_{1}}^{v_{i_{1}}} \cdots X_{i_{m}}^{v_{i_{m}}} Y_{j_{1}}^{-u_{j_{1}}} \cdots Y_{j_{n}}^{-u_{j_{n}}}$, com $\left\{i_{1}, \ldots, i_{m}, j_{1}, \ldots, j_{n}\right\} \in$ I e $u_{j_{1}}<$ $0, \ldots, u_{j_{n}}<0$. Notemos que para $\mathbf{v}$, cada coordenada $v_{i}$, será expoente de $X$ ou de $Y$, de acordo com a definição de $\mathbf{X}_{\mathbf{v}}$. Logo um índice $i$ de $X$, não aparecerá como índice de $Y$, e vice-versa. Novamente pela definição de $\mathbf{X}_{\mathbf{v}}$, temos que $v_{i_{1}} \geq 1$. 
Vamos escolher $t_{i_{1}}=X_{i_{1}} Y_{i_{1}} \in A_{0}$ e calcular $\left[\mathbf{X}_{\mathbf{v}}, t_{i_{1}}\right]$.

$$
\begin{aligned}
& {\left[\mathbf{X}_{\mathbf{v}}, t_{i_{1}}\right]=\left[X_{i_{1}}^{v_{i_{1}}} \cdots X_{i_{m}}^{v_{i_{m}}} Y_{j_{1}}^{-u_{j_{1}}} \cdots Y_{j_{n}}^{-u_{j_{n}}}, X_{i_{1}} Y_{i_{1}}\right]} \\
& \stackrel{\text { Obs.1.1.1.2 }}{=} X_{i_{1}}\left[X_{i_{1}}^{v_{i_{1}}} \cdots X_{i_{m}}^{v_{i_{m}}} Y_{j_{1}}^{-u_{j_{1}}} \cdots Y_{j_{n}}^{-u_{j_{n}}}, Y_{i_{1}}\right]+\left[X_{i_{1}}^{v_{i_{1}}} \cdots X_{i_{m}}^{v_{i_{m}}} Y_{j_{1}}^{-u_{j_{1}}} \cdots Y_{j_{n}}^{-u_{j_{n}}}, X_{i_{1}}\right] Y_{i_{1}} \\
& \stackrel{\text { Obs.1.1.1.1 }}{=} X_{i_{1}}\left(\left[X_{i_{1}}^{v_{i_{1}}} \cdots X_{i_{m}}^{v_{i_{m}}}, Y_{i_{1}}\right] Y_{j_{1}}^{-u_{j_{1}}} \cdots Y_{j_{n}}^{-u_{j_{n}}}+X_{i_{1}}^{v_{i_{1}}} \cdots X_{i_{m}}^{v_{i_{m}}}\left[Y_{j_{1}}^{-u_{j_{1}}} \cdots Y_{j_{n}}^{-u_{j_{n}}}, Y_{i_{1}}\right]\right) \\
& +\left(X_{i_{1}}^{v_{i_{1}}} \cdots X_{i_{m}}^{v_{i_{m}}}\left[Y_{j_{1}}^{-u_{j_{1}}} \cdots Y_{j_{n}}^{-u_{j_{n}}}, X_{i_{1}}\right]+\left[X_{i_{1}}^{v_{i_{1}}} \cdots X_{i_{m}}^{v_{i_{m}}}, X_{i_{1}}\right] Y_{j_{1}}^{-u_{j_{1}}} \cdots Y_{j_{n}}^{-u_{j_{n}}}\right) Y_{i_{1}}
\end{aligned}
$$

Temos que $\left[X_{i_{1}}^{v_{i_{1}}} \cdots X_{i_{m}}^{v_{i_{m}}}, X_{i_{1}}\right]=\left[Y_{j_{1}}^{-u_{j_{1}}} \cdots Y_{j_{n}}^{-u_{j_{n}}}, Y_{i_{1}}\right]=0$. Então:

$$
\left[\mathbf{X}_{\mathbf{v}}, t_{i_{1}}\right]=\underbrace{X_{i_{1}}\left[X_{i_{1}}^{v_{i_{1}}} \cdots X_{i_{m}}^{v_{i_{m}}}, Y_{i_{1}}\right] Y_{j_{1}}^{-u_{j_{1}}} \cdots Y_{j_{n}}^{-u_{j_{n}}}}_{1}+\underbrace{X_{i_{1}}^{v_{i_{1}}} \cdots X_{i_{m}}^{v_{i_{m}}}\left[Y_{j_{1}}^{-u_{j_{1}}} \cdots Y_{j_{n}}^{-u_{j_{n}}}, X_{i_{1}}\right] Y_{i_{1}}}_{2}
$$

Simplificando 1:

$$
\begin{aligned}
X_{i_{1}}[ & \left.X_{i_{1}}^{v_{i_{1}}} \cdots X_{i_{m}}^{v_{i_{m}}}, Y_{i_{1}}\right] Y_{j_{1}}^{-u_{j_{1}}} \cdots Y_{j_{n}}^{-u_{j_{n}}}= \\
& X_{i_{1}} X_{i_{1}}^{v_{i_{1}}} \cdots X_{i_{m}}^{v_{i_{m}}} Y_{i_{1}} Y_{j_{1}}^{-u_{j_{1}}} \cdots Y_{j_{n}}^{-u_{j_{n}}}- \\
& X_{i_{1}} Y_{i_{1}} X_{i_{1}}^{v_{i_{1}}} \cdots X_{i_{m}}^{v_{i_{m}}} Y_{j_{1}}^{-u_{j_{1}}} \cdots Y_{j_{n}}^{-u_{j_{n}}} \\
= & X_{i_{1}} X_{i_{1}}^{v_{i_{1}}} Y_{i_{1}} X_{i_{2}}^{v_{i_{2}}} \cdots X_{i_{m}}^{v_{i_{m}}} Y_{j_{1}}^{-u_{j_{1}}} \cdots Y_{j_{n}}^{-u_{j_{n}}}- \\
& X_{i_{1}} Y_{i_{1}} X_{i_{1}}^{v_{i_{1}}} X_{i_{2}}^{v_{i_{2}}} \cdots X_{i_{m}}^{v_{i_{m}}} Y_{j_{1}}^{-u_{j_{1}}} \cdots Y_{j_{n}}^{-u_{j_{n}}} \\
= & X_{i_{1}}\left[X_{i_{1}}^{v_{i_{1}}}, Y_{i_{1}}\right] X_{i_{2}}^{v_{i_{2}}} \cdots X_{i_{m}}^{v_{i_{m}}} Y_{j_{1}}^{-u_{j_{1}}} \cdots Y_{j_{n}}^{-u_{j_{n}}}
\end{aligned}
$$

Pela Proposição 1.1.1, item 2, $\left[X_{i_{1}}^{v_{i_{1}}}, Y_{i_{1}}\right]=-\left[Y_{i_{1}}, X_{i_{1}}^{v_{i_{1}}}\right]=-v_{i_{1}} X_{i_{1}}^{v_{i_{1}}-1}$. Assim

$$
X_{i_{1}}\left[X_{i_{1}}^{v_{i_{1}}} \cdots X_{i_{m}}^{v_{i_{m}}}, Y_{i_{1}}\right] Y_{j_{1}}^{-u_{j_{1}}} \cdots Y_{j_{n}}^{-u_{j_{n}}}=-v_{i_{1}} X_{i_{1}}^{v_{i_{1}}} \cdots X_{i_{m}}^{v_{i_{m}}} Y_{j_{1}}^{-u_{j_{1}}} \cdots Y_{j_{n}}^{-u_{j_{n}}}=-v_{i_{1}} \mathbf{X}_{\mathbf{v}}
$$

Agora vamos simplificar 2:

$$
\begin{aligned}
& X_{i_{1}}^{v_{i_{1}}} \cdots X_{i_{m}}^{v_{i_{m}}}\left[Y_{j_{1}}^{-u_{j_{1}}} \cdots Y_{j_{n}}^{-u_{j_{n}}}, X_{i_{1}}\right] Y_{i_{1}}= \\
& X_{i_{1}}^{v_{i_{1}}} \cdots X_{i_{m}}^{v_{i_{m}}} Y_{j_{1}}^{-u_{j_{1}}} \cdots Y_{j_{n}}^{-u_{j_{n}}} X_{i_{1}} Y_{i_{1}}- \\
& X_{i_{1}}^{v_{i_{1}}} \cdots X_{i_{m}}^{v_{i_{m}}} X_{i_{1}} Y_{j_{1}}^{-u_{j_{1}}} \cdots Y_{j_{n}}^{-u_{j_{n}}} Y_{i_{1}} \\
& \doteq X_{i_{1}}^{v_{i_{1}}} \cdots X_{i_{m}}^{v_{i_{m}}} Y_{j_{1}}^{-u_{j_{1}}} \cdots Y_{j_{n}}^{-u_{j_{n}}} X_{i_{1}} Y_{i_{1}}- \\
& X_{i_{1}}^{v_{i_{1}}} \cdots X_{i_{m}}^{v_{i_{m}}} Y_{j_{1}}^{-u_{j_{1}}} \cdots Y_{j_{n}}^{-u_{j_{n}}} X_{i_{1}} Y_{i_{1}}=0
\end{aligned}
$$

Na passagem $\dagger$ usamos o fato que $i_{1} \neq j_{k}, 1 \leq k \leq n$. Portanto $\left[\mathbf{X}_{\mathbf{v}}, t_{i_{1}}\right]=-v_{i_{1}} \mathbf{X}_{\mathbf{v}} \neq 0$, pois $v_{i_{1}} \geq 1$. Então $-a_{\mathbf{v}} v_{i_{1}} \mathbf{X}_{\mathbf{v}} \neq 0$. Temos que:

$$
\left[Z, t_{i_{1}}\right]=a_{\mathbf{v}}\left[\mathbf{X}_{\mathbf{v}}, t_{i_{1}}\right]+\left[Z^{\prime}, t_{i_{1}}\right]=-a_{\mathbf{v}} v_{i_{1}} \mathbf{X}_{\mathbf{v}}+\left[Z^{\prime}, t_{i_{1}}\right]
$$

Como $\operatorname{deg}\left(Z^{\prime}\right)<\operatorname{deg}\left(\mathbf{X}_{\mathbf{v}}\right)$, pelo Teorema 1.1.3, item 3, temos que $\operatorname{deg}\left[Z^{\prime}, t_{i_{1}}\right] \leq \operatorname{deg}\left(Z^{\prime}\right)+$ $2-2<\operatorname{deg}\left(\mathbf{X}_{\mathbf{v}}\right)$. Então $\left[Z^{\prime}, t_{i_{1}}\right]$ não cancela o elemento $-a_{\mathbf{v}} v_{i_{1}} \mathbf{X}_{\mathbf{v}}$. Portanto $\left[Z, t_{i_{1}}\right] \neq 0$.

Chegamos assim à uma contradição.

Já que nenhum elemento $\mathbf{X}_{\mathbf{v}}, \operatorname{com} \mathbf{v} \neq 0$, comuta com todos os elementos de $A_{0}$, segue que $A_{0}$ é uma subálgebra comutativa maximal em $A$. Em outras palavras, $A_{0}$ é uma subálgebra comutativa maximal em $A$, porque não existe elemento de $A \backslash A_{0}$ que comute com todos 
os elementos de $A_{0}$, isto é, não existe uma subálgebra comutativa $A^{\prime}$ de $A$ que contenha $A_{0}$ propriamente.

Nós provamos que os geradores da álgebra $A_{0}$, os $t_{i}, i \in \mathrm{I}$, são algebricamente independentes sobre $k$. Além disso, provamos também que eles comutam entre si. Sendo assim podemos dizer que:

$$
A_{0} \cong k\left[t_{i} \mid i \in \mathrm{I}\right]
$$

ou seja, $A_{0}$ pode ser vista como uma $k$-álgebra comutativa dos polinômios em infinitas variáveis $t_{i}, i \in \mathrm{I}$.

Agora vamos definir alguns conceitos importantes e resultados envolvendo o anel dos polinômios em $n$ variáveis comutativas $k\left[x_{1}, \ldots, x_{n}\right]$ com coeficientes em $k$. Observamos que esse anel na verdade é uma $k$-álgebra, pois contém $k$ como subanel (subanel dos polinômios com grau 0). Para mais detalhes indicamos [SZ91] (Capítulos I e II) e [Jac80] (Capítulo 7).

Observação 1.3.1. Quando dizemos que um polinômio $f\left(x_{1}, \ldots, x_{n}\right)=\sum a_{i_{1} \ldots i_{n}} x_{1}^{i_{1}} \cdots x_{n}^{i_{n}}$ em $k\left[x_{1}, \ldots, x_{n}\right]$ é não nulo, significa que algum coeficiente $a_{i_{1} \ldots i_{n}}$ deste polinômio é não nulo. Se o corpo não tiver característica 0 pode acontecer que um polinômio não nulo assuma o valor zero em todos os pontos de $k^{n}$. Por exemplo, o polinômio de uma variável $f(x)=x^{2}+x$ $\in \mathbb{F}_{2}[x]$ é zero quando avaliamos nos dois pontos $\{\overline{0}, \overline{1}\}$ de $\mathbb{F}_{2}$, mas $f$ não é o polinômio nulo. $k$.

Temos que $k^{n}$ é o $k$-espaço vetorial $n$-dimensional das $n$-uplas $\left(a_{1}, \ldots, a_{n}\right)$, em que $a_{i} \in$

Para qualquer subconjunto $Z$ de $k^{n}$, o ideal

$$
I(Z)=\left\{f \in k\left[x_{1}, \ldots, x_{n}\right] \mid f\left(a_{1}, \ldots, a_{n}\right)=0 \text { para todos }\left(a_{1}, \ldots, a_{n}\right) \in Z\right\}
$$

de todos os polinômios que se anulam identicamente em $Z$ é um ideal no anel dos polinômios $k\left[x_{1}, \ldots, x_{n}\right]$. Ele é chamado de ideal de $Z$.

Para qualquer conjunto $S \subseteq k\left[x_{1}, \ldots, x_{n}\right]$, podemos definir um subconjunto algébrico $V(S)$ sobre $k$ como o seguinte subconjunto de $k^{n}$ :

$$
V(S)=\left\{\left(a_{1}, \ldots, a_{n}\right) \in k^{n} \mid f\left(a_{1}, \ldots, a_{n}\right)=0 \text { para todo } f \in S\right\}
$$

Notemos que $V(S)=V(\langle S\rangle)$, então todos os conjuntos algébricos são conjuntos de zeros de algum ideal, assim só precisamos considerar os conjuntos algébricos $V(I)$ para algum ideal $I \subseteq k\left[x_{1}, \ldots, x_{n}\right]$.

Pelo Teorema da Base de Hilbert (veja Capítulo 2, Teorema 2.3.2), se $I \subseteq k\left[x_{1}, \ldots, x_{n}\right]$ é um ideal, então ele é finitamente gerado, isto é, $I=\left\langle f_{1}, \ldots, f_{m}\right\rangle$, para alguns polinômios $f_{i}, 1 \leq i \leq m$. Em particular, todo conjunto algébrico é um conjunto de zeros comuns de uma quantidade finita de polinômios, para qualquer subconjunto $S \subseteq k\left[x_{1}, \ldots, x_{n}\right]$, ou seja, $V(S)=V(\langle S\rangle)=V\left(\left\langle f_{1}, \ldots, f_{m}\right\rangle\right)=V\left(f_{1}, \ldots, f_{m}\right)$.

Podemos dar um significado geométrico à alguns quocientes do anel de polinômios. Seja $Z$ um conjunto algébrico em $k^{n}$. Se tomarmos qualquer polinômio $f\left(x_{1}, \ldots, x_{n}\right)$, podemos considerá-lo como uma $k$-função avaliada em $k^{n}$, por avaliação em cada ponto $p=$ $\left(a_{1}, \ldots, a_{n}\right)$, isto é, $f(p)$ para todo $p \in k^{n}$. Podemos restringir tal função polinomial $f$ ao subconjunto $Z \subseteq k^{n}$, e tomar uma função em $Z$. Mas diferentes polinômios $f, g \in k\left[x_{1}, \ldots, x_{n}\right]$, podem acabar restringindo a mesma função em $Z$. Isto acontece se, e somente se, $f-g$ se anula identicamente em $Z$ se, e somente se, $f-g \in I(Z)$ se, e somente se, $\bar{f}=\bar{g}$ em $k\left[x_{1}, \ldots, x_{n}\right] / I(Z)$. Então $k\left[x_{1}, \ldots, x_{n}\right] / I(Z)$ é simplesmente o anel de funções em $Z$ que são restrições de funções polinomiais em $k^{n}$, é chamado de anel das funções $k$-regulares e é denotado por $k[Z]$ (não deve ser confundido com um anel de polinômios na variável $Z$ ). 
Se $I \subseteq k\left[x_{1}, \ldots, x_{n}\right]$ é um ideal próprio, sua intersecção com os polinômios de grau $0, k$ $\subseteq k\left[x_{1}, \ldots, x_{n}\right]$, é $\{0\}$. Então o anel quociente $k\left[x_{1}, \ldots, x_{n}\right] / I$ também contém $k$ como um subanel, e então o quociente é uma $k$-álgebra. Anéis quocientes da forma $C=k\left[x_{1}, \ldots, x_{n}\right] / I$, em que $I$ é um ideal próprio, são chamados de $k$-álgebras de tipo finito e sua dimensão como um $k$-espaço vetorial é enumerável. As classes de equivalências $\bar{x}_{i}$ em $C$ serão denotadas por $y_{i}$ por conveniência, e chamadas de funçôes coordenadas. Claramente, $\overline{f\left(x_{1}, \ldots, x_{n}\right)}=$ $f\left(y_{1}, \ldots, y_{n}\right)$ pela definição das operações de anel em $C$, e então estes $y_{i}$ 's geram $C$ como uma $k$-álgebra, isto é, todo elemento de $C$ pode ser escrito como um polinômio nas variáveis $y_{i}$ 's com coeficientes em $k$. Se $f\left(x_{1}, \ldots, x_{n}\right) \in I$, por definição, a classe de equivalência $\overline{f\left(x_{1}, \ldots, x_{n}\right)}=0$ em $C$, isto é, $f\left(y_{1}, \ldots, y_{n}\right)=0$. Reciprocamente, se $f\left(y_{1}, \ldots, y_{n}\right)=0$, então $f \in I$. Logo $I$ é precisamente o conjunto das "restrições" ou "relações" que medem a "distância" do anel $C$ para o anel de polinômios $k\left[x_{1}, \ldots, x_{n}\right]$.

Vamos caracterizar os ideais maximais de $k\left[x_{1}, \ldots, x_{n}\right]$.

Um ideal $I$ em um anel comutativo $R$ é chamado de ideal maximal se ele é um ideal próprio, e não contém nenhum ideal próprio além dele mesmo. Todo ideal próprio de um anel comutativo $R$ está contido em algum ideal maximal em $R$, pelo Lema de Zorn.

Como resultado da álgebra comutativa temos que um ideal próprio $I$ em um anel comutativo $R$ é maximal se, e somente se, $R / I$ é um corpo. De fato, se $I \subseteq J$ para algum ideal $J \subseteq R$, então o conjunto $J / I=\{\bar{a} \mid a \in J\}$ é um ideal de $R$; $J$ é um ideal próprio de $R$ se, e somente se, $J / I$ é um ideal próprio de $R / I$; além disso, $J=I$ se, e somente se, $J / I=\{0\}$; portanto $I$ é um ideal maximal em $R$ se, e somente se, $R / I$ não contém ideais próprios além de $\{0\}$.

Pelo Teorema Fundamental da Álgebra, todo polinômio de grau $\geq 1$ em $\mathbb{C}[x]$ tem uma raiz em $\mathbb{C}$. Como consequências deste teorema: todo polinômio de grau $\geq 1$ em $\mathbb{C}[x]$ é um produto de polinômios lineares e os únicos ideais maximais em $\mathbb{C}[x]$ são os ideais $\langle x-a\rangle$, para algum $a \in \mathbb{C}$. Então os ideais maximais em $\mathbb{C}[x]$ estão em correspondência biunívoca com $\mathbb{C}$.

Agora vamos reunir alguns fatos importantes da teoria dos corpos que serão relevantes posteriormente.

Se $k \subseteq K$ é um extensão de corpos algébrica (isto é, todo elemento de $K$ é algébrico sobre $k$ ) e $k$ é algebricamente fechado então $k=K$.

Se $k \subseteq K$ é uma extensão de corpos tal que $K$ é uma $k$-álgebra de tipo finito, e $k$ é um conjunto não enumerável, então $K$ é uma extensão algébrica de $k$. De fato, como todo elemento de $k$ é certamente algébrico sobre $k$, basta tomar $a \in K \backslash k$ e considerar o conjunto $\left\{(a-c)^{-1} \mid c \in k\right\}$; observamos que $(a-c) \neq 0$, para todo $c \in k$ e todos os elementos do conjunto acima são distintos; a cardinalidade do conjunto é a mesma cardinalidade de $k$, ou seja, o conjunto é um subconjunto não enumerável de $K$; como a dimensão de $K$ como $k$-espaço vetorial é enumerável, o conjunto é linearmente dependente; segue que $a$ é algébrico sobre $k$. Como consequência desse fato: se $k \subseteq K$ é uma extensão de corpos tais que $K$ é uma $k$-álgebra de tipo finito e $k$ é algebricamente fechado e não enumerável, então $k=K$.

Uma extensão $K$ de $k$ é transcendente se ela não é algébrica, isto é, se $K$ contém elementos que são transcendentes sobre $k$. Se $K$ é obtida de $k$ por adjunção de elementos de algum conjunto transcendente $L$, então $K$ é dita ser uma extensão puramente transcendente de $k$.

A seguinte proposição é geralmente chamada de pequeno Nullstellensatz, embora seja equivalente ao grande Nullstellensatz. Na Alemanha, "Nullstellensatz" significa "Teorema dos Zeros".

Proposição 1.3.5 (Hilbert's Nullstellensatz I). Seja $k$ um corpo algebricamente fechado. Então o ideal $I \subseteq k\left[x_{1}, \ldots, x_{n}\right]$ é maximal se, e somente se, $I=\left\langle x_{1}-a_{1}, \ldots, x_{n}-a_{n}\right\rangle$ para 
alguns $a_{i} \in k$. Em outras palavras, os ideais maximais estão em correspondência biunívoca com os pontos de $k^{n}$.

Demonstração. Vamos verificar primeiro que os ideais $I=\left\langle x_{1}-a_{1}, \ldots, x_{n}-a_{n}\right\rangle$ são maximais. Para isso precisamos mostrar que $R / I$ é um corpo, em que $R=k\left[x_{1}, \ldots, x_{n}\right]$. Se $f\left(x_{1}, \ldots, x_{n}\right)$ é qualquer polinômio em $R$, podemos reescrevê-lo como

$$
f\left(\left(x_{1}-a_{1}\right)+a_{1}, \ldots,\left(x_{n}-a_{n}\right)+a_{n}\right)
$$

Agora qualquer potência $\left(\left(x_{i}-a_{i}\right)+a_{i}\right)^{n_{i}}$ pode ser escrita na forma $\left(x_{i}-a_{i}\right) g_{i}+a_{i}^{n_{i}}$ (por expansão binomial), em que $g_{i}$ é algum polinômio em $x_{i}$. Substituindo estes em $f$, temos pela expansão de Taylor de $f$ :

$$
f\left(x_{1}, \ldots, x_{n}\right)=f\left(a_{1}, \ldots, a_{n}\right)+\sum_{i=1}^{n} h_{i}\left(x_{1}, \ldots, x_{n}\right)\left(x_{i}-a_{i}\right)
$$

em que $h_{i} \in R$. O segundo termo do lado direito da igualdade pertence claramente ao ideal $I=\left\langle x_{1}-a_{1}, \ldots, x_{n}-a_{n}\right\rangle$. Isto mostra que todo elemento em $R=k\left[x_{1}, \ldots, x_{n}\right]$ é congruente ao elemento $f\left(a_{1}, \ldots, a_{n}\right) \in k(\bmod I)$. Temos também que todo elemento $a \in k$ $\subseteq R$ é claramente congruente somente à ele mesmo $(\bmod I)$ (pois $I \cap k=\{0\})$. Então $R / I$ é simplesmente o corpo $k$, e portanto $I$ é maximal.

Reciprocamente, seja $I \subseteq R=k\left[x_{1}, \ldots, x_{n}\right]$ um ideal maximal. Pela definição $R / I$ é uma $k$-álgebra de tipo finito, contendo $k$, e é um corpo. Denotemos por $K$. Então $k \subseteq K$ é uma extensão de corpos, e pelas hipóteses concluímos que $k=K$. Denotando as classes de equivalências de $x_{i}$ por $\bar{x}_{i} \in K=R / I$, segue que $\bar{x}_{i}=a_{i} \in k$ para todo $i=1, \ldots, n$. Dizer que $\bar{x}_{i}=a_{i}$ em $K$, por definição, significa que $x_{i}-a_{i} \in I$ para todo $i$. Então o ideal $\left\langle x_{1}-a_{1}, \ldots, x_{n}-a_{n}\right\rangle \subseteq I$. Entretanto, já provamos que o ideal $\left\langle x_{1}-a_{1}, \ldots, x_{n}-a_{n}\right\rangle$ é maximal. Então ele é igual à $I$, que é um ideal próprio.

Generalizando esse resultado para $A_{0} \cong k\left[t_{i} \mid i \in \mathrm{I}\right]$, temos que o ideal de $A_{0}$ gerado pelos elementos $t_{i}-p_{i}, p_{i} \in k, i \in \mathrm{I}$, é maximal em $A_{0}$. Então, para $\mathbf{p} \in k^{\mathrm{I}}$, denotamos por $\mathfrak{m}_{\mathbf{p}} \mathrm{o}$ ideal maximal em $A_{0}$ gerado por $t_{i}-p_{i}, i \in \mathrm{I}$, isto é, $\mathfrak{m}_{\mathbf{p}}=\left\langle t_{i}-p_{i}\right\rangle_{i \in \mathrm{I}}$.

O próximo lema sobre os ideais maximais da $k$-álgebra $A_{0}$ tem uma relação com o "Hilbert's Nullstellensatz I".

Lema 1.3.1. Todo ideal maximal em $A_{0}$ tem a forma $\mathfrak{m}_{\mathbf{p}}$, para algum $\mathbf{p} \in k^{\mathrm{I}}$.

Demonstração. Seja m um ideal maximal em $A_{0}$. Então $A_{0} / \mathfrak{m}$ é um corpo. $\operatorname{Logo}\left(A_{0} / \mathfrak{m}\right) / k$ é uma extensão de corpos.

Como $k$ é algebricamente fechado, todo polinômio com coeficientes em $k$, possui todas as suas raízes em $k$. Sendo assim, todos os elementos algébricos de $A_{0} / \mathfrak{m}$ sobre $k$ pertencem à $k$. Seja $z \in\left(A_{0} / \mathfrak{m}\right) \backslash k$. O elemento $z$ é transcendente sobre $k$, pois se existisse um polinômio não nulo com coeficientes em $k$ tal que $z$ fosse raiz deste polinômio, então $z \in k$, pois $k$ é algebricamente fechado. Então a extensão de corpos $k \hookrightarrow A_{0} / \mathfrak{m}$ é um isomorfismo ou é uma extensão puramente transcendente.

Suponhamos que $k \cong A_{0} / \mathfrak{m}$. Para $t_{i}, i \in \mathrm{I}$, existe $p_{i} \in k$ tal que $p_{i}$ é congruente a $t_{i}$ $(\bmod \mathfrak{m})$, ou seja, $t_{i}-p_{i} \in \mathfrak{m}$, para $i \in \mathrm{I}$. Então $\mathfrak{m}_{\mathbf{p}}=\left\langle t_{i}-p_{i}\right\rangle_{i \in \mathrm{I}} \subseteq \mathfrak{m}$, em que $\mathbf{p} \in k^{\mathrm{I}}$. Como $\mathfrak{m}$ é maximal em $A_{0}$, por definição $\mathfrak{m} \neq A_{0}$. Então $\mathfrak{m}_{\mathbf{p}} \subseteq \mathfrak{m}$ implica que $\mathfrak{m}_{\mathbf{p}}=\mathfrak{m}$, pois $\mathfrak{m}_{\mathbf{p}}$ é maximal em $A_{0}$.

Se a extensão é puramente transcendente, escolhemos $z \in\left(A_{0} / \mathfrak{m}\right) \backslash k$ (que é transcendente sobre $k$ ). Notemos que $(z-c) \neq 0$, para qualquer $c \in k$. Temos que o conjunto formado 
pelos elementos $(z-c)^{-1}$, com $c$ percorrendo $k$, é linearmente independente sobre $k$. De fato: suponhamos que $\left(z-c_{1}\right)^{-1}, \ldots,\left(z-c_{n}\right)^{-1}$, com $c_{1}, \ldots, c_{n} \in k$, são linearmente dependentes sobre $k$; então existem $a_{1}, \ldots, a_{n} \in k$, nem todos nulos tal que $a_{1}\left(z-c_{1}\right)^{-1}+\ldots+a_{n}\left(z-c_{n}\right)^{-1}=$ 0 . Consideremos $f(x) \in k[x]$, tal que $f(x)=a_{1}\left(x-c_{1}\right)^{-1}+\ldots+a_{n}\left(x-c_{n}\right)^{-1}$. Temos que $f(z)=0$ e como $k$ é algebricamente fechado, temos que $z \in k$, isso é uma contradição. Isso significa que a $k$-dimensão de $A_{0} / \mathfrak{m}$ é no mínimo $|k|$, isto é, $\geq|k|$. Por outro lado, a $k$-dimensão de $A_{0}$ é $|\mathrm{I}|$ e $|\mathrm{I}|<|k|$ (condição imposta no início da seção), ou seja, a $k$-dimensão de $A_{0} / \mathfrak{m}$ é $<|k|$, uma contradição.

Portanto o resultado segue.

\section{4 Âlgebra de Weyl generalizada}

As álgebras de Weyl generalizadas foram introduzidas por Bavula em [Bav92a].

Vimos na Seção 1.1 que a $n$-ésima álgebra de Weyl $A_{n}(k)$ é a $k$-álgebra associativa unital com geradores $x_{1}, \ldots, x_{n}, \partial_{1}, \ldots, \partial_{n}$ que satisfazem as seguintes relações:

$$
\left[x_{i}, x_{j}\right]=\left[\partial_{i}, \partial_{j}\right]=0 \quad \text { e } \quad\left[\partial_{i}, x_{j}\right]=\delta_{i j}
$$

para $1 \leq i, j \leq n$.

Nesta seção $n$ pode ser um inteiro positivo ou infinito enumerável.

Pela definição dada por Bavula em [Bav92a]: seja $D$ um anel, $\sigma$ um automorfismo de $D$ e a um elemento central de $D$ (ou seja, a pertence ao centro de $D$ ); a álgebra de Weyl generalizada $D(\sigma, a)$ de grau 1 é o anel gerado por $D$ e por duas indeterminadas $X$ e $Y$ sujeitas às relações:

$$
X d=\sigma(d) X \quad \text { e } \quad Y d=\sigma^{-1}(d) Y
$$

para todo $d \in D$ e

$$
Y X=a \quad \text { e } \quad X Y=\sigma(a)
$$

Em muitos casos $D$ é uma álgebra sobre um corpo $k$ e $\sigma$ é um automorfismo de álgebras. Então, neste caso, $D(\sigma, a)$ é uma $k$-álgebra.

Exemplo 1.4.1. Seja $D=k[H]$, a k-álgebra dos polinômios na variável $H$. Sejam $\sigma$ o único automorfismo de $D$ que verifica $\sigma(H)=H-1$ e $a=\alpha H+\beta$ um polinômio de grau 1 . A álgebra de Weyl generalizada $D(\sigma, a)$ que obtemos tem relações:

$$
\begin{gathered}
H Y=Y H-Y \quad ; \quad X H=H X-X \\
Y X=\alpha H+\beta \quad ; \quad X Y=\alpha H+\beta-\alpha
\end{gathered}
$$

Neste caso temos a relação $X Y=Y X-\alpha$. Desta maneira temos uma apresentação de $D(\sigma, a)$ com geradores $X, Y$ e uma relação $X Y=Y X-\alpha$. Concluímos que a álgebra de Weyl generalizada de grau 1 que corresponde a esses dados é isomorfa à álgebra de Weyl clássica $A_{1}=k\langle\partial, x \mid \partial x-x \partial=1\rangle$.

Agora vamos à realização de $A_{n}(k)$ como uma álgebra de Weyl generalizada de grau $n$ $D(\underline{\sigma}, \underline{a})$ (veja [Bav92a], [BB00] ou [BBF04]).

Definição 1.4.1. Para um grupo $G$, seja $A(G)=\{\alpha \in$ Aut $(G) \mid x \alpha(x)=\alpha(x)$ x para todo $x \in$ $G\}$. Os automorfismos que pertencem a $A(G)$ são chamados de automorfismos comutantes. 
Para definirmos a álgebra de Weyl generalizada de grau $n$ partimos de uma álgebra associativa unital $D$; uma $n$-upla de elementos centrais $\underline{a}=\left(a_{1}, \ldots, a_{n}\right)$ de $D$; e uma $n$-upla de automorfismos comutantes $\underline{\sigma}=\left(\sigma_{1}, \ldots, \sigma_{n}\right)$ de $D\left(\sigma_{i} \sigma_{j}=\sigma_{j} \sigma_{i}\right.$, para todo $\left.i, j\right)$ tais que $\sigma_{i}\left(a_{j}\right)=a_{j}$ se $i \neq j$. A álgebra $D(\underline{\sigma}, \underline{a})$ é gerada sobre $D$ por elementos $X_{i}, Y_{i}, i=1, \ldots, n$, que satisfazem as relações:

$$
\begin{gathered}
X_{i} d=\sigma_{i}(d) X_{i} \quad, \quad Y_{i} d=\sigma_{i}^{-1}(d) Y_{i} \\
Y_{i} X_{i}=a_{i} \quad, \quad X_{i} Y_{i}=\sigma_{i}\left(a_{i}\right) \\
{\left[X_{i}, X_{j}\right]=\left[Y_{i}, Y_{j}\right]=0 \quad, \quad 1 \leq i, j \leq n} \\
{\left[Y_{i}, X_{j}\right]=0 \quad, \quad 1 \leq i \neq j \leq n}
\end{gathered}
$$

para todo $d \in D$.

Os elementos $\tilde{t}_{i}=\partial_{i} x_{i}$ na álgebra de Weyl $A_{n}(k)$ geram uma álgebra polinomial $D=$ $k\left[\tilde{t_{1}}, \ldots, \tilde{t_{n}}\right]$. Observamos que na Seção 1.3 definimos $t_{i}=x_{i} \partial_{i}$, logo $\tilde{t_{i}}=t_{i}+1$, em que 1 é o operador identidade. Tomando $\underline{a}=\left(a_{1}, \ldots, a_{n}\right)$, em que $a_{i}=\tilde{t}_{i} \in D$, definindo $X_{i}=x_{i}$ e $Y_{i}=\partial_{i}$, e considerando $\underline{\sigma}=\left(\sigma_{1}, \ldots, \sigma_{n}\right)$ como a $n$-upla dos automorfismos comutantes de $D$ dados por $\sigma_{i}\left(\tilde{t_{j}}\right)=\tilde{t_{j}}-\delta_{i j} \cdot 1$, obtemos uma realização de $A_{n}(k)$ como a álgebra de Weyl generalizada $D(\underline{\sigma}, \underline{a})$.

A álgebra $A_{\infty}$ pode ser vista como uma álgebra de Weyl generalizada de posto infinito.

Exemplo 1.4.2. A álgebra de Weyl quântica $A_{1}(q)=\langle\partial, x \mid \partial x-q x \partial=1\rangle$ sobre $k$, em que $q \neq 0 \in k$, é uma álgebra de Weyl generalizada $A_{1}(q) \cong D(\sigma, a)$, em que $D=k[H]$ (a álgebra dos polinômios na variável $H), a=H, \sigma$ é definido da seguinte maneira $\sigma(H)=q^{-1}(H-1)$, e usamos as identificações $x \leftrightarrow X, \partial \leftrightarrow Y$ e $\partial x \leftrightarrow H$.

Exemplo 1.4.3. $O$ plano quântico $\Lambda=k\langle X, Y \mid X Y=q Y X\rangle$, em que $q \neq 0 \in k$, é uma álgebra de Weyl generalizada $\Lambda \cong D(\sigma, a)$, em que $D=k[H]$ (a álgebra dos polinômios na variável $H), a=H, \sigma$ é definido da seguinte maneira $\sigma(H)=q H$, e usamos as identificações $X \leftrightarrow X, Y \leftrightarrow Y$ e $Y X \leftrightarrow H$.

Seja uma $k$-álgebra $\mathcal{A}=\bigotimes_{i=1}^{n} \mathcal{A}_{i}$ o produto tensorial (sobre $k$ ) de álgebras de Weyl generalizadas $\mathcal{A}_{i}=D_{i}\left(\sigma_{i}, a_{i}\right)$ de grau 1 sobre um corpo $k$ algebricamente fechado, em que $D_{i}=k\left[H_{i}\right]$ são anéis de polinômios em uma variável; $\sigma_{i}$ é um automorfismo de $D_{i}$ tal que $\sigma_{i}\left(H_{i}\right)=\lambda_{i} H_{i}+\mu_{i}$, em que $\lambda_{i} \neq 0, \mu_{i} \in k$; e $a_{i}$ é um elemento não nulo de $D_{i}$. Então $\mathcal{A}$ é uma álgebra de Weyl generalizada de grau $n$.

Ou seja,

$$
\mathcal{A}=\left(\bigotimes_{i=1}^{n} D_{i}\right)\left(\underline{\sigma}=\left(\sigma_{1}, \ldots, \sigma_{n}\right), \underline{a}=\left(a_{1}, \ldots, a_{n}\right)\right)
$$

é uma álgebra de Weyl generalizada de grau $n$, com anel básico $D=\bigotimes_{i=1}^{n} D_{i}=k\left[H_{1}, \ldots, H_{n}\right]$, o anel de polinômios em $n$ variáveis; com $a_{i}=H_{i}$, para $i=1, \ldots, n$; e com automorfismos $\left\{\sigma_{i}: \sigma_{i}\left(H_{i}\right)=\lambda_{i} H_{i}+\mu_{i}, \sigma_{i}\left(H_{j}\right)=H_{j}\right.$, se $\left.i \neq j\right\}$.

Vejamos dois exemplos dessa construção:

Exemplo 1.4.4. A n-ésima álgebra de Weyl $A_{n}(k)=A_{1} \otimes \ldots \otimes A_{1}$ ( $n$ vezes), é a álgebra de Weyl generalizada $D(\underline{\sigma}, \underline{a})$ de grau $n$ em que $D=k\left[H_{1}, \ldots, H_{n}\right],\left\{a_{i} \in D \mid a_{i}=H_{i}, 1 \leq\right.$ $i \leq n\}$ e $\left\{\sigma_{i} \mid \sigma_{i}\left(H_{j}\right)=H_{j}-\delta_{i j}, 1 \leq i, j \leq n\right\}$.

Exemplo 1.4.5. A álgebra de Weyl quântica de grau $n$

$$
A_{n}(q)=A_{1}(q) \otimes \ldots \otimes A_{1}(q) \quad(n \text { vezes }) \quad, q \neq 0 \in k
$$


é outro exemplo de uma álgebra de Weyl generalizada que é o produto tensorial de álgebras de Weyl generalizadas de grau 1.

Suponhamos que $\mathcal{A}=D(\underline{\sigma}, \underline{a}), \ldots, \mathcal{A}^{\prime}=D^{\prime}\left(\underline{\sigma^{\prime}}, \underline{a^{\prime}}\right)$ são álgebras de Weyl generalizadas de grau $n, \ldots, m$ respectivamente. Então o produto tensorial entre elas (sobre $k$ )

$$
\mathcal{A} \otimes \ldots \otimes \mathcal{A}^{\prime}=D \otimes \ldots \otimes D^{\prime}\left(\underline{\sigma} \cup \ldots \cup \underline{\sigma^{\prime}}, \underline{a} \cup \ldots \cup \underline{a^{\prime}}\right)
$$

é uma álgebra de Weyl generalizada de grau $n+\cdots+m$ com anel base $D \otimes \ldots \otimes D^{\prime}$.

A álgebra oposta $\mathcal{A}^{o p}$ de uma álgebra de Weyl generalizada também é uma álgebra de Weyl generalizada:

$$
\mathcal{A}^{o p}=D^{o p}\left(\underline{\sigma}^{-1}, \underline{\sigma(a)}\right)
$$

em que $\underline{\sigma}^{-1}=\left(\sigma_{i}^{-1}\right)_{i}$ e $\sigma(a)=\left(\sigma_{i}\left(a_{i}\right)\right)_{i}$.

Podemos graduar uma álgebra de Weyl generalizada. Seja $\mathcal{A}=D(\underline{\sigma}, \underline{a})$ uma álgebra de Weyl generalizada de grau $n$. Para qualquer vetor $\underline{w}=\left(w_{1}, \ldots, w_{n}\right) \in \mathbb{Z}^{n}$, tomemos $v_{\underline{w}}=v_{w_{1}}(1) \ldots v_{w_{n}}(n)$, em que para quaisquer $1 \leq i \leq n$ e $m>0$ :

$$
v_{m}(i)=X_{i}^{m} \quad, \quad v_{-m}(i)=Y_{i}^{m} \quad \text { e } \quad v_{0}(i)=1 .
$$

No caso $n=1$ escrevemos $v_{m}$ para $v_{m}(1)$.

Segue da definição de uma álgebra de Weyl generalizada que

$$
\mathcal{A}=\bigoplus_{\underline{w} \in \mathbb{Z}^{n}} \mathcal{A}_{\underline{w}}
$$

é uma $\mathbb{Z}^{n}$-álgebra graduada, com espaços homogêneos $\mathcal{A}_{\underline{w}}=D v_{\underline{w}}=v_{\underline{w}} D$, tais que $\mathcal{A}_{\underline{w}} \mathcal{A}_{\underline{u}} \subseteq$ $\mathcal{A}_{\underline{w}+\underline{u}}$, para todos $\underline{w}, \underline{u} \in \mathbb{Z}^{n}$.

Segue que $\mathcal{A}$ é um $D$-módulo livre à esquerda e à direita.

Observamos que na Seção 1.3 definimos o elemento

$$
\mathbf{X}_{\mathbf{v}}=\prod_{i: v_{i}>0} X_{i}^{v_{i}} \prod_{i: v_{i}<0} Y_{i}^{-v_{i}}
$$

para $\mathbf{v} \in \mathbb{Z}_{f}^{\mathrm{I}}$, e provamos que o conjunto formado por elementos dessa forma, variando o vetor $\mathbf{v}$, é uma base de $A=A_{k, \mathrm{I}}$ como $A_{0}$-módulo à direita e à esquerda. Na verdade, aquela ideia é praticamente uma generalização desta graduação descrita acima.

\subsection{Referências}

Grande parte da Seção 1.1 foi baseada em [Cou95] [Capítulos 1,2 e 3]. A Seção 1.2 foi baseada em [Cou95][Capítulo 2]. A Seção 1.3 foi baseada em [FGM14][Seções 2.1 e 2.2]. A Seção 1.4 foi baseada em [Bav92a], [BBF04] e [BB00]. 


\section{Capítulo 2}

\section{Módulos sobre a álgebra de Weyl}

\subsection{Módulos sobre a álgebra de Weyl $A_{n}$}

Podemos pensar que o conceito de um módulo sobre um anel é uma generalização da noção de espaço vetorial sobre um corpo, pois em um módulo os escalares pertencem à um anel arbitrário.

Vamos começar lembrando a definição de um $R$-módulo. Seja $R$ um anel. Um $R$-módulo à esquerda $M$ é um subgrupo abeliano aditivo, munido da ação $R \times M \longrightarrow M$, tal que $(a, m) \mapsto a \cdot m$, que satisfaz:

1. $a \cdot(m+n)=a \cdot m+a \cdot n$;

2. $(a+b) \cdot n=a \cdot n+b \cdot n$;

3. $(a b) \cdot m=a \cdot(b \cdot m)$;

4. $1_{R} \cdot m=m$.

para todos $a, b \in R$ e para todos $m, n \in M$. Em alguns casos, chamaremos simplesmente de $R$-módulo. Também escrevemos am para denotar o elemento $a \cdot m$ do $R$-módulo $M$.

No Capítulo 1, vimos que a álgebra de Weyl $A_{n}$ é um subanel de $\operatorname{End}_{k}\left(k\left[x_{1}, \ldots, x_{n}\right]\right)$, em que $k\left[x_{1}, \ldots, x_{n}\right]$ é o anel dos polinômios em $n$ variáveis comutativas. Deduz-se daí que $k\left[x_{1}, \ldots, x_{n}\right]$ é um $A_{n}$-módulo à esquerda, em que a ação de $x_{i}$ em $k\left[x_{1}, \ldots, x_{n}\right]$ é dada pela multiplicação pela variável $x_{i}$, enquanto $\partial_{i}$ age pela diferenciação com respeito à $x_{i}$.

Definição 2.1.1. Seja $R$ um anel. Um $R$-módulo $M$ não nulo é irredutível, ou simples, se os únicos submódulos de $M$ são os triviais.

Definição 2.1.2. Seja $M$ um R-módulo à esquerda. Um elemento $u \in M$ é um elemento de torção se $\operatorname{ann}_{R}(u)=\{a \in R \mid a u=0\}$ (aniquilador de $u$ ) é um ideal à esquerda de $R$ não nulo. Se todo elemento de $M$ é de torção, então $M$ é dito ser um módulo de torção.

Observação 2.1.1. A definição dada acima é encontrada em [Cou95]. Observamos que alguns autores definem um módulo de torção sobre um dominio de integridade, definem um submódulo de torção $t(M)$, e então $M$ é módulo de torção se $t(M)=M$.

Lema 2.1.1. Seja $R$ um anel e $M$ um $R$-módulo à esquerda irredutível.

1. Se $0 \neq u \in M$, então $M \cong R / \operatorname{ann}_{R}(u)$.

2. Se $R$ não é um anel de divisão, então $M$ é um R-módulo de torção. 
Demonstração. 1. Considere a aplicação $\phi: R \longrightarrow M$ definida por $\phi\left(1_{R}\right)=1_{R} u=u$. Esta aplicação é um homomorfismo de $R$-módulos. Como $u \neq 0$ e $M$ é irredutível, $\phi$ é sobrejetora, pois o $R$-submódulo à esquerda de $M$ gerado por $u$ é igual à $M$, ou seja, $i m(\phi)=M$. Temos também que $\operatorname{ker}(\phi)=a n n_{R}(u)$. De fato: se $b \in a n n_{R}(u)$, então $\phi(b)=b u=0$, isto é, $b \in \operatorname{ker}(\phi)$; reciprocamente, se $a \in \operatorname{ker}(\phi)$ então $\phi(a)=0=a u$, isto é, $a \in \operatorname{ann}_{R}(u)$. Pelo Primeiro Teorema do Isomorfismo para $R$-módulos, temos que $R / \operatorname{ker}(\phi) \cong i m(\phi)$. Portanto $R / \operatorname{ann}_{R}(u) \cong M$.

2. Suponhamos que $\operatorname{ann}_{R}(u)=0$, para algum $0 \neq u \in M$. Segue do item 1 . que $M \cong R$. Como $M$ é irredutível, os únicos ideais à esquerda de $R$ são os triviais. Mas neste caso $R$ é um anel de divisão, contradizendo a hipótese. Então $a n_{R}(u) \neq 0$. Portanto $M$ é um $R$-módulo de torção.

Vamos aplicar este resultado para o $A_{n}$-módulo $k\left[x_{1}, \ldots, x_{n}\right]$.

Proposição 2.1.1. $k\left[x_{1}, \ldots, x_{n}\right]$ é um $A_{n}$-módulo de torção irredutível. Além disso,

$$
k\left[x_{1}, \ldots, x_{n}\right] \cong A_{n} / \sum_{i=1}^{n} A_{n} \cdot \partial_{i}
$$

Demonstração. Primeiramente, $1 \in k$ é claramente um gerador de $k\left[x_{1}, \ldots, x_{n}\right]$ como $A_{n^{-}}$ módulo à esquerda. Agora suponha que $f \neq 0$ é um polinômio de $k\left[x_{1}, \ldots, x_{n}\right]$ e considere o submódulo $A_{n} \cdot f$ (submódulo gerado por $f$ ). Seja $x_{1}^{i_{1}} \cdots x_{n}^{i_{n}}$ um monômio com maior grau possível, de acordo com a ordem lexicográfica, dentre todos os monômios que aparecem em $f$ com coeficientes não nulos. Seja $a$ o seu coeficiente. Então $\partial_{1}^{i_{1}} \ldots \partial_{n}^{i_{n}}(f)=i_{1} ! \ldots i_{n}$ ! $a$, que é uma constante não nula pertencente ao submódulo gerado por $f$. Assim $k\left[x_{1}, \ldots, x_{n}\right] \subseteq A_{n} \cdot f$. Portanto $A_{n} \cdot f=k\left[x_{1}, \ldots, x_{n}\right]$. Concluímos, que os únicos submódulos de $k\left[x_{1}, \ldots, x_{n}\right]$ são os triviais, ou seja, $k\left[x_{1}, \ldots, x_{n}\right]$ é um $A_{n}$-módulo irredutível.

Já provamos que $A_{n}$ não é um anel de divisão no Capítulo 1. Portanto segue do Lema 2.1.1(2), que $k\left[x_{1}, \ldots, x_{n}\right]$ é um $A_{n}$-módulo de torção.

Agora 1 é um elemento não nulo de $k\left[x_{1}, \ldots, x_{n}\right]$ que é aniquilado por $\partial_{1}, \ldots, \partial_{n}$. Então o ideal à esquerda $J$ de $A_{n}$ gerado por $\partial_{1}, \ldots, \partial_{n}, J=\sum_{i=1}^{n} A_{n} \cdot \partial_{i}$, está contido em ann $n_{A_{n}}(1)$. Reciprocamente, seja $P \in$ ann $_{A_{n}}(1)$. Como $P \in A_{n}, P$ pode ser escrito na forma $f+Q$, em que $Q \in J$ e $f \in k\left[x_{1}, \ldots, x_{n}\right]$. Então $0=P \cdot 1=Q \cdot 1+f \cdot 1=f \cdot 1$, implica que $f=0$. Assim $P=Q \in J$. Concluímos que $J=a n n_{A_{n}}$ (1). O isomorfismo segue do Lema 2.1.1(1).

Podemos generalizar essa ideia como segue. Escolha $g_{1}, \ldots, g_{n} \in k\left[x_{1}, \ldots, x_{n}\right]$ e considere o ideal à esquerda $J$ de $A_{n}$ gerado por $\partial_{1}-g_{1}, \ldots, \partial_{n}-g_{n}$. Todo elemento de $A_{n}$ é da forma $f+P$, para $f \in k\left[x_{1}, \ldots, x_{n}\right]$ e $P \in J$ (veja [Cou95], Capítulo 5, Exercício 4.1). Então a aplicação $\psi: A_{n} / J \longrightarrow k\left[x_{1}, \ldots, x_{n}\right]$ definida por $\psi(f+J)=f$ é um isomorfismo de $k$-espaços vetoriais. Embora a ação dos $x$ 's seja preservada pelo isomorfismo, a aplicação $\psi$ não é um isomorfismo de $A_{n}$-módulos. De fato, se $f$ é um polinômio, então

$$
\partial_{i} \cdot(f+J)=\frac{\partial f}{\partial x_{i}}+f \cdot \partial_{i}+J=\frac{\partial f}{\partial x_{i}}+f \cdot g_{i}+J
$$

Logo $\psi\left(\partial_{i} \cdot(f+J)\right)=\partial_{i} \cdot f+g_{i} \cdot f=\left(\partial_{i}+g_{i}\right) \cdot f$, tal que o lado direito deve ser calculado em $k\left[x_{1}, \ldots, x_{n}\right]$ com a sua ação natural. Portanto $\psi$ não preserva a ação de $\partial_{i}$.

O $A_{n}$-módulo $A_{n} / J$ é irredutível; a prova é similar a demonstração da Proposição 2.1.1.

Um outro módulo que está intimamente relacionado ao $k\left[x_{1}, \ldots, x_{n}\right]$ é o $A_{n}$-módulo $A_{n} / \sum_{i=1}^{n} A_{n} \cdot x_{i}$. Como um $k$-espaço vetorial ele é isomorfo à $k[\partial]=k\left[\partial_{1}, \ldots, \partial_{n}\right]$, o conjunto 
dos polinômios nas variáveis $\partial_{1}, \ldots, \partial_{n}$. Usando este isomorfismo, podemos identificar a ação do $A_{n}$ diretamente em $k\left[\partial_{1}, \ldots, \partial_{n}\right]$ : os $\partial$ 's agem por multiplicação, enquanto a ação de $x_{i}$ em $\partial_{j}$ é dada por $-\delta_{i j} \cdot 1$. Além das semelhanças óbvias, os $A_{n}$-módulos $k\left[\partial_{1}, \ldots, \partial_{n}\right]$ e $k\left[x_{1}, \ldots, x_{n}\right]$ estão relacionados de modo mais profundo que vai ser explicado posteriormente na Proposição 2.1.3.

Seja $R$ um anel e $M$ um $R$-módulo à esquerda. Suponhamos que $\sigma$ é um automorfismo de $R$. Podemos definir um novo $R$-módulo à esquerda $M_{\sigma}$, do seguinte modo:

Definição 2.1.3. Seja $M_{\sigma}$ um conjunto, tal que $M_{\sigma}=M$ como grupos abelianos e existe uma ação de $R$ em $M_{\sigma}$ definida por $a \bullet u=\sigma(a) u$, para todos $a \in R, u \in M$. $M_{\sigma}$ é um $R$-módulo à esquerda, chamado de módulo torcido de $M$ por $\sigma$.

A diferença entre $M$ e $M_{\sigma}$, reside na ação de $R$ em $M_{\sigma}$. Sendo assim, $M_{\sigma}$ herda muitas das propriedades de $M$.

Proposição 2.1.2. Seja $R$ um anel, $M$ um $R$-módulo à esquerda e $\sigma$ um automorfismo de R. Então:

1. $M_{\sigma}$ é irredutivel se, e somente se, $M$ é irredutivel.

2. $M_{\sigma}$ é um módulo de torção se, e somente se, $M$ é um módulo de torção.

3. Se $N$ é um submódulo de $M$ então $(M / N)_{\sigma} \cong M_{\sigma} / N_{\sigma}$.

4. Seja $J$ um ideal à esquerda de R. Seja $\sigma(J)=\{\sigma(r) \mid r \in J\}$. Então $\sigma(J)$ é um ideal à esquerda de $R$ e $(R / J)_{\sigma} \cong R / \sigma^{-1}(J)$.

Demonstração. 1. Um $R$-módulo $M$ é irredutível se, e somente se, para todo $0 \neq u \in M$, $R \cdot u=M$ se, e somente se, para quaisquer $u, v \in M$, não nulos, existe $a \in R$ tal que $a u=v$. Esta equação se traduz como $\sigma^{-1}(a) \bullet u=\sigma\left(\sigma^{-1}(a)\right) u=a u=v$ em $M_{\sigma}$, o que prova 1 .

2. Similarmente, para $a \in R, u \in M$, a equação $a u=0$ em $M$, torna-se $\sigma^{-1}(a) \bullet u=$ $\sigma\left(\sigma^{-1}(a)\right) u=a u=0$ em $M_{\sigma}$, o que prova 2.

3. É uma aplicação imediata do Primeiro Teorema do Isomorfismo para $R$-módulos.

4. Como $\sigma$ é um automorfismo de $R$, segue que $\sigma(J)$ é um ideal à esquerda de $R$, pois $\sigma(J) \neq \varnothing$; se $\sigma\left(r_{1}\right), \sigma\left(r_{2}\right) \in \sigma(J)$, temos que $\sigma\left(r_{1}\right)+\sigma\left(r_{2}\right)=\sigma\left(r_{1}+r_{2}\right) \in \sigma(J)$, pois $J$ é um ideal à esquerda de $R$; além disso, para $b \in R, \sigma(r) \in \sigma(J)$, temos que $b \sigma(r)=\sigma\left(\sigma^{-1}(b)\right) \sigma(r)=\sigma\left(\sigma^{-1}(b) r\right) \in \sigma(J)$, pois $\sigma^{-1}(b) r \in J$. Seja $\phi: R \longrightarrow(R / J)_{\sigma}$ um homomorfismo de $R$-módulos definido por $\phi\left(1_{R}\right)=1_{R}+J$. Se $b \in R$, então $\phi(b)=$ $b \bullet \phi\left(1_{R}\right)=\sigma(b)\left(1_{R}+J\right)=\sigma(b)+J$. Assim $\phi$ é sobrejetiva. Além disso, $b \in \operatorname{ker}(\phi) \Leftrightarrow$ $\phi(b)=\sigma(b)+J=0+J \Leftrightarrow \sigma(b) \in J \Leftrightarrow b \in \sigma^{-1}(J)$. Então $\operatorname{ker}(\phi)=\sigma^{-1}(J)$. Portanto 4 segue do Primeiro Teorema do Isomorfismo para $R$-módulos.

Vamos aplicar esta construção para $A_{n}$. Um importante exemplo é a Transformação de Fourier. Seja $\mathcal{F}$ um automorfismo de $A_{n}$ definido por $\mathcal{F}\left(x_{i}\right)=\partial_{i}$ e $\mathcal{F}\left(\partial_{i}\right)=-x_{i}$ (veja [Cou95], Capítulo 1, Exercício 4.8). Seja $M$ um $A_{n}$-módulo à esquerda. O módulo torcido $M_{\mathcal{F}}$ é chamado de Transformação de Fourier de $M$. A razão para o nome é que $\mathcal{F}$ transforma um operador diferencial com coeficientes constantes em um polinômio.

Proposição 2.1.3. A Transformação de Fourier de $k\left[x_{1}, \ldots, x_{n}\right]$ é $k\left[\partial_{1}, \ldots, \partial_{n}\right]$. 
Demonstração. Segue da Proposição 2.1.1 que $k\left[x_{1}, \ldots, x_{n}\right] \cong A_{n} / J$, em que $J=\sum_{i=1}^{n} A_{n} \cdot \partial_{i}$. Como $\mathcal{F}^{-1}(J)=\sum_{i=1}^{n} A_{n} \cdot \mathcal{F}^{-1}\left(\partial_{i}\right)=\sum_{i=1}^{n} A_{n} \cdot x_{i}$, podemos aplicar a Proposição 2.1.2(4), e temos que:

$$
\left(k\left[x_{1}, \ldots, x_{n}\right]\right)_{\mathcal{F}} \cong\left(A_{n} / J\right)_{\mathcal{F}} \cong A_{n} / \mathcal{F}^{-1}(J)=A_{n} / \sum_{i=1}^{n} A_{n} \cdot x_{i}
$$

Temos que $A_{n} / \sum_{i=1}^{n} A_{n} \cdot x_{i} \cong k\left[\partial_{1}, \ldots, \partial_{n}\right]$, como $k$-espaços vetoriais, portanto a Transformação de Fourier de $k\left[x_{1}, \ldots, x_{n}\right]$ é $k\left[\partial_{1}, \ldots, \partial_{n}\right]$, ou seja, $\left(k\left[x_{1}, \ldots, x_{n}\right]\right)_{\mathcal{F}} \cong k\left[\partial_{1}, \ldots, \partial_{n}\right]$.

Segue da Proposição 2.1.2(1) e da Proposição 2.1.3, que $k\left[\partial_{1}, \ldots, \partial_{n}\right]$ é um $A_{n}$-módulo irredutível.

\subsection{Módulos graduados e filtrados}

Como vimos no Capítulo 1, Subseção 1.1.3, podemos definir um grau para os elementos da álgebra de Weyl. Usando esse grau, podemos construir um anel comutativo $S_{n}=g r^{\mathcal{B}} A_{n}$, que funciona como uma sombra de $A_{n}$. Podemos então desenhar um esboço do que $A_{n}$ realmente se parece. Este é o melhor método que nós temos para entender a estrutura de $A_{n}$ e seus módulos.

\subsubsection{Anéis e módulos graduados}

Uma importante característica do anel de polinômios é que ele admite uma função grau. Nós queremos generalizar e formalizar o que significa para uma álgebra ter um grau. Isso leva à definição de anéis graduados. Estes anéis encontram sua justificativa em geometria algébrica, mais precisamente em geometria algébrica projetiva. Definiremos anéis graduados sem assumir comutatividade.

Definição 2.2.1. Seja $R$ uma $k$-álgebra. $R$ é $k$ - álgebra graduada se existem $k$-subespaços vetoriais $R_{i}, i \in \mathbb{N}$, tais que:

1. $R=\bigoplus_{i \in \mathbb{N}} R_{i}$,

2. $R_{i} \cdot R_{j} \subseteq R_{i+j}$.

Os $R_{i}$ são chamados de componentes homogêneas de $R$. Os elementos de $R_{i}$ são os elementos homogêneos de grau $i$. Se $R_{i}=\{0\}$ quando $i<0$, dizemos que a graduação é positiva. Vamos considerar somente graduações positivas.

O exemplo mais importante de uma álgebra graduada é o anel de polinômios $k\left[x_{1}, \ldots, x_{n}\right]$. Os monômios $x_{1}^{k_{1}} \ldots x_{n}^{k_{n}} \operatorname{com} k_{1}+\ldots+k_{n}=m$, formam uma base da componente homogênea de grau $m$.

Os mais importantes anéis graduados que aparecem em geometria algébrica são quocientes de anéis de polinômios. Eles são construídos como segue.

Definição 2.2.2. Seja $R$ uma k-álgebra graduada. Um ideal bilateral $I$ de $R$ é um ideal graduado se $I=\bigoplus_{i>0}\left(I \cap R_{i}\right)$.

Então, um ideal graduado é gerado por elementos homogêneos. A recíproca também é verdadeira: um ideal gerado por elementos homogêneos será graduado. 
Definição 2.2.3. Sejam $R=\bigoplus_{i \geq 0} R_{i}$ e $S=\bigoplus_{i \geq 0} S_{i}$, k-álgebras graduadas. Um homomorfismo de k-álgebras $\phi: R \rightarrow S$ é um homomorfismo graduado se $\phi\left(R_{i}\right) \subseteq S_{i}$, para todo $i \geq 0$.

Assim, um homomorfismo graduado é aquele que preserva o grau. Os conceitos de homomorfismo graduado e ideal graduado estão relacionados, como mostra o próximo resultado:

Proposição 2.2.1. Sejam $R=\bigoplus_{i \geq 0} R_{i}$ e $S=\bigoplus_{i \geq 0} S_{i}$, k-álgebras graduadas.

1. O kernel de um homomorfismo graduado de k-álgebras $\phi: R \rightarrow S$ é um ideal bilateral graduado de $R$.

2. Se I é um ideal bilateral graduado de $R$ então $R / I$ é uma k-álgebra graduada.

Demonstração. 1. Suponha que $\phi$ é um homomorfismo graduado. O kernel de $\phi$ é um ideal bilateral de $R$. Seja $a=a_{0} \oplus \ldots \oplus a_{s} \in R$, um elemento do kernel de $\phi$. Então $\phi(a)=\phi\left(a_{0}\right)+\ldots+\phi\left(a_{s}\right)$. Como $\phi$ é graduado, $\phi\left(a_{i}\right) \in S_{i}$, para cada $i=0, \ldots, s$. Então a soma é direta, isto é, $\phi(a)=\oplus_{i=0}^{s} \phi\left(a_{i}\right)$. Como $\phi(a)=0$, temos que $\phi\left(a_{i}\right)=0$, para cada $i$. Então $a_{i} \in \operatorname{ker}(\phi)$, para $i=0, \ldots, s$. Assim $\operatorname{ker}(\phi)=\oplus_{i \geq 0}\left(\operatorname{ker}(\phi) \cap R_{i}\right)$. Portanto 1. está provado.

2. Seja $I$ um ideal bilateral graduado de $R$. Então podemos decompor o anel quociente $R / I$ em uma soma direta de $k$-espaços vetoriais,

$$
R / I \cong \bigoplus_{i \geq 0}\left(R_{i} /\left(I \cap R_{i}\right)\right)
$$

Se $a_{i} \in R_{i}$ e $a_{j} \in R_{j}$ então $\left(a_{i}+I\right)\left(a_{j}+I\right)=a_{i} a_{j}+I$ corresponde à um elemento de $R_{i+j} /\left(I \cap R_{i+j}\right)$, sob esse isomorfismo. Então $R / I$ é um anel graduado (isto é, uma $k$-álgebra graduada), o que prova 2 .

Isto nos fornece uma maneira de gerar exemplos de anéis graduados. Sejam $f_{1}, \ldots, f_{k}$ polinômios homogêneos em $k\left[x_{1}, \ldots, x_{n}\right]$. O quociente $k\left[x_{1}, \ldots, x_{n}\right] /\left\langle f_{1}, \ldots, f_{k}\right\rangle$ é um anel graduado, em que $\left\langle f_{1}, \ldots, f_{k}\right\rangle$ é o ideal de $k\left[x_{1}, \ldots, x_{n}\right]$ gerado por $f_{1}, \ldots, f_{k}$.

Uma álgebra graduada admite um tipo especial de módulo.

Definição 2.2.4. Seja $R=\bigoplus_{i \geq 0} R_{i}$ uma k-álgebra graduada. Um R-módulo à esquerda $M$

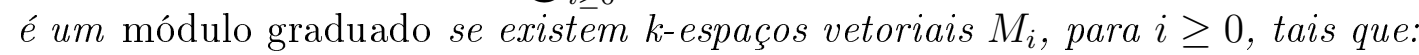

1. $M=\bigoplus_{i \geq 0} M_{i}$,

2. $R_{i} \cdot M_{j} \subseteq M_{i+j}$.

Os $M_{i}$ são as componentes homogêneas de grau $i$ de $M$. Observamos que a definição de módulo graduado depende da estrutura graduada escolhida para a álgebra $R$.

Podemos definir submódulos graduados e homomorfismos de módulos graduados imitando as definições correspondentes para anéis graduados.

Definição 2.2.5. Sejam $R$ uma k-álgebra graduada e $M, M^{\prime} R$-módulos à esquerda graduados. Um submódulo $N$ de $M$ é um submódulo graduado se $N=\bigoplus_{i \geq 0}\left(N \cap M_{i}\right)$. Um homomorfismo de R-módulos $\theta: M \rightarrow M^{\prime}$ é um homomorfismo graduado se $\theta\left(M_{i}\right) \subseteq M_{i}^{\prime}$. 
Segue que o $\operatorname{ker}(\theta)$ é um submódulo graduado e que o módulo quociente $M / N$ é um $R$-módulo graduado. A demonstração é similar a prova da Proposição 2.2.1.

Isto permite produzir exemplos de módulos graduados. Seja $R=\bigoplus_{i>0} R_{i}$ uma $k$-álgebra graduada e $R^{n}$ o $R$-módulo à esquerda livre de posto $n$. Este módulo tem uma graduação natural, sua $m$-ésima componente homogênea é o espaço vetorial

$$
\sum_{i_{1}+\ldots+i_{n}=m}\left(R_{i_{1}} \oplus \cdots \oplus R_{i_{n}}\right)
$$

Se $L$ é um submódulo graduado de $R^{n}$, então $R^{n} / L$ é um módulo à esquerda graduado finitamente gerado. A importância dos anéis graduados é explicada na próxima subseção.

\subsubsection{Anéis filtrados}

No Capítulo 1, Subseção 1.1.3, introduzimos o grau de um operador na álgebra de Weyl $A_{n}$. Entretanto, este grau não pode ser usado para graduar o anel $A_{n}$. O problema é que um elemento como $\partial_{1} x_{1}$ deveria ser homogêneo de grau 2, mas é igual à $x_{1} \partial_{1}+1$, que não é homogêneo. Para usar esse grau efetivamente, devemos generalizar anéis graduados, obtendo anéis filtrados.

Definição 2.2.6. Seja $R$ uma k-álgebra. Uma familia $\mathcal{F}=\left\{F_{i}\right\}_{i \geq 0}$ de $k_{\text {-espaços vetoriais é }}$ uma filtração de $R$ se

1. $F_{0} \subseteq F_{1} \subseteq F_{2} \subseteq \cdots \subseteq R$,

2. $R=\bigcup_{i \geq 0} F_{i}$,

3. $F_{i} \cdot F_{j} \subseteq F_{i+j}$.

Se uma álgebra tem uma filtração, ela é chamada de álgebra filtrada. Por convenção $F_{j}=$ $\{0\}$, se $j<0$.

Agora vamos mostrar que toda álgebra graduada é filtrada. Suponha que $G=\bigoplus_{i \geq 0} G_{i}$ é uma $k$-álgebra graduada. Considere os $k$-espaços vetoriais $F_{r}=\bigoplus_{i=0}^{r} G_{i}$. Claramente $F_{r} \subseteq F_{r+1}$ e a união de todos os $F_{r}$ é $G$. Como

$$
F_{r} \cdot F_{s}=\left(\bigoplus_{i=0}^{r} G_{i}\right)\left(\bigoplus_{j=0}^{s} G_{j}\right)=\bigoplus_{i+j \leq r+s} G_{i} \cdot G_{j}
$$

e $G_{i} \cdot G_{j} \subseteq G_{i+j}$, temos que $F_{r} \cdot F_{s} \subseteq F_{r+s}$. Então $\left\{F_{r}\right\}_{r \geq 0}$ é uma filtração de $G$. Por outro lado, existem álgebras filtradas que não tem uma graduação natural. Isto acontece com a álgebra de Weyl $A_{n}$, que possui diferentes filtrações. Vamos apresentar dois exemplos.

A primeira filtração de $A_{n}$ que será discutida é a filtração de Bernstein. É a filtração definida usando o grau dos operadores de $A_{n}$. Seja $r \in \mathbb{N}$. Denote por $B_{r}$ o conjunto de todos os operadores de $A_{n}$ de grau $\leq r$. Esses são subespaços vetoriais de $A_{n}$. As condições 1. e 2. de uma filtração são claramente satisfeitas pelos $B_{r}$ 's, enquanto que 3. é consequência do Teorema 1.1.3(2), ou seja, para $D \in B_{s}$ e $D^{\prime} \in B_{t}$, o grau de $D D^{\prime}$ é $\leq s+t$, isto é, $D D^{\prime}$ $\in B_{s+t}$. Então $\mathcal{B}=\left\{B_{r}\right\}_{r \in \mathbb{N}}$ é uma filtração de $A_{n}$. Também escrevemos $\mathcal{B}\left(A_{n}\right)$ ou $B_{r}\left(A_{n}\right)$.

A filtração de Bernstein tem uma característica muito especial: cada $B_{r}$ é um espaço vetorial de dimensão finita. Uma base para $B_{r}$ é determinada por monômios $x^{\alpha} \partial^{\beta}$ com $|\alpha|+|\beta| \leq r$. Em particular, $B_{0}=k$ e $\left\{1, x_{1}, \ldots, x_{n}, \partial_{1}, \ldots, \partial_{n}\right\}$ é a base de $B_{1}$. 
Um outro importante exemplo de filtração para $A_{n}$ é a filtração ordem, denotada por $\mathcal{C}$. Como no Capítulo 1, Subseção 1.1.4, denote por $C_{r}$ o $k$-espaço vetorial de todos os operadores de ordem $\leq r$ em $A_{n}$. É claro que a propriedade 1. de uma filtração é válida para $\mathcal{C}$, a propriedade 2. é consequência do Teorema 1.1.5 e a propriedade 3. é uma consequência da Proposição 1.1.7 (Capítulo 1, Subseção 1.1.4). Note que $C_{0}=k\left[x_{1}, \ldots, x_{n}\right]$ é um $k$-espaço vetorial de dimensão infinita. Apesar desta desvantagem, a filtração ordem tem a vantagem de que, ao contrário da filtração de Bernstein, é bem definida para outros anéis de operadores diferenciais.

\subsection{3 Álgebra graduada associada}

Nós podemos usar uma filtração de uma álgebra para construir uma graduação desta álgebra.

Seja $R$ uma $k$-álgebra. Suponha que $\mathcal{F}=\left\{F_{i}\right\}_{i \in \mathbb{N}}$ é uma filtração de $R$. Como primeiro passo na construção da álgebra graduada, introduziremos a aplicação símbolo de ordem $r$, que é a projeção canônica de $k$-espaços vetoriais

$$
\sigma_{r}: F_{r} \rightarrow F_{r} / F_{r-1}
$$

Então para um operador $d \in F_{r}$, o símbolo $\sigma_{r}(d)$ é não nulo se, e somente se, $d \notin F_{r-1}$.

Considere agora o $k$-espaço vetorial

$$
g r^{\mathcal{F}} R=\bigoplus_{i \geq 0}\left(F_{i} / F_{i-1}\right)
$$

Queremos fazer a graduação da $k$-álgebra $R$. Para isto é suficiente definir uma multiplicação de dois elementos homogêneos, e estender por linearidade. Um elemento homogêneo de $g r^{\mathcal{F}} R$ é da forma $\sigma_{r}(a)$ para algum $a \in F_{r}$. Seja $\sigma_{m}(b)$, para $b \in F_{m}$, um outro elemento homogêneo, e defina o produto entre eles por

$$
\sigma_{r}(a) \sigma_{m}(b)=\sigma_{r+m}(a b)
$$

Vamos verificar que este produto está bem definido, isto é, o elemento $\sigma_{r+m}(a b)$ em $F_{r+m} / F_{r+m-1}$ não depende da escolha de $a$ e $b$. Seja $a_{1}$ um outro elemento em $F_{r}$ tal que $\sigma_{r}\left(a_{1}\right)=\sigma_{r}(a)$ em $F_{r} / F_{r-1}$. Então $\sigma_{r}\left(a_{1}-a\right)=0$ em $F_{r} / F_{r-1}$. Isto significa que $a_{1}-a \in F_{r-1}$. Como $\mathcal{F}$ é uma filtração, $a_{1} b-a b \in F_{r+m-1}$. Logo $\sigma_{r+m}\left(a_{1} b-a b\right)=0 \mathrm{em} F_{r+m} / F_{r+m-1}$, ou seja, $\sigma_{r+m}\left(a_{1} b\right)=\sigma_{r+m}(a b)$ em $F_{r+m} / F_{r+m-1}$. Similarmente, podemos mostrar que $\sigma_{r+m}(a b)$ não depende da escolha de $b$.

Então $g r^{\mathcal{F}} R$ com esta multiplicação é uma $k$-álgebra graduada, com componentes homogêneas $F_{i} / F_{i-1}$. De fato: sejam $x \in F_{r} / F_{r-1}$ e $y \in F_{m} / F_{m-1}$. Temos que, existem $a \in F_{r}$ tal que $\sigma_{r}(a)=x$ e $b \in F_{m}$ tal que $\sigma_{m}(b)=y$. Assim $x y=\sigma_{r}(a) \sigma_{m}(b)=\sigma_{r+m}(a b)$. Portanto $x y$ $\in F_{r+m} / F_{r+m-1}$, como queríamos. Dizemos que $g r^{\mathcal{F}} R$ é a álgebra graduada de $R$ associada com a filtração $\mathcal{F}$.

Vejamos agora uma importante aplicação deste resultado. Seja $S_{n}=g r^{\mathcal{B}} A_{n}$, a álgebra graduada de $A_{n}$ associada com a filtração de Bernstein $\mathcal{B}$.

Teorema 2.2.1. A álgebra graduada $S_{n}$ é isomorfa ao anel de polinômios sobre $k$ em $2 n$ variáveis.

Demonstração. Vamos dividir a prova em alguns passos. Para $i=1, \ldots, n$, sejam $y_{i}=\sigma_{1}\left(x_{i}\right)$ 
e $y_{i+n}=\sigma_{1}\left(\partial_{i}\right)$. Se $y_{j}=\sigma_{1}\left(x_{j}\right)$, observamos que:

$$
y_{j}^{m}=\underbrace{y_{j} \cdots y_{j}}_{m \text { vezes }}=\sigma_{1}\left(x_{j}\right) \ldots \sigma_{1}\left(x_{j}\right)=\sigma_{(1+\cdots+1)}\left(x_{j} \cdots x_{j}\right)=\sigma_{m}\left(x_{j}^{m}\right)
$$

Da mesma forma, se $y_{j+n}=\sigma_{1}\left(\partial_{j}\right)$, então $y_{j+n}^{m}=\sigma_{m}\left(\partial_{j}^{m}\right)$.

Primeiro passo: $S_{n}$ é gerada por $y_{1}, \ldots, y_{2 n}$ como uma $k$-álgebra.

Como $S_{n}$ é uma $k$-álgebra graduada, é suficiente provar para elementos homogêneos de $S_{n}$. Mas, um elemento homogêneo de $S_{n}$ é da forma $\sigma_{r}(d)$, para algum $d \in A_{n}$ com grau $r$. Agora $d \in B_{r}$, ou seja, $d$ é uma combinação linear de monômios $x^{\alpha} \partial^{\beta}$, com $|\alpha|+|\beta| \leq r$. Se $|\alpha|+|\beta|=r$, então

$$
\begin{aligned}
\sigma_{r}\left(x^{\alpha} \partial^{\beta}\right) & =\sigma_{|\alpha|+|\beta|}\left(x^{\alpha} \partial^{\beta}\right)=\sigma_{|\alpha|}\left(x^{\alpha}\right) \sigma_{|\beta|}\left(\partial^{\beta}\right) \\
& =\left(\sigma_{\alpha_{1}+\ldots+\alpha_{n}}\left(x_{1}^{\alpha_{1}} \cdots x_{n}^{\alpha_{n}}\right)\right)\left(\sigma_{\beta_{1}+\ldots+\beta_{n}}\left(\partial_{1}^{\beta_{1}} \cdots \partial_{n}^{\beta_{n}}\right)\right) \\
& =\left(\sigma_{\alpha_{1}}\left(x_{1}^{\alpha_{1}}\right) \cdots \sigma_{\alpha_{n}}\left(x_{n}^{\alpha_{n}}\right)\right)\left(\sigma_{\beta_{1}}\left(\partial_{1}^{\beta_{1}}\right) \cdots \sigma_{\beta_{n}}\left(\partial_{n}^{\beta_{n}}\right)\right) \\
& =\left(y_{1}^{\alpha_{1}} \cdots y_{n}^{\alpha_{n}}\right)\left(y_{n+1}^{\beta_{1}} \cdots y_{2 n}^{\beta_{n}}\right)
\end{aligned}
$$

Então $\sigma_{r}(d)$ é uma combinação linear de monômios em $y_{1}, \ldots, y_{2 n}$ de grau $r$, como queríamos provar.

Segundo passo: $S_{n}$ é um anel comutativo.

Como $S_{n}$ é gerada por $y_{1}, \ldots, y_{2 n}$, precisamos somente mostrar que esses elementos comutam em $S_{n}$. Para $i=1, \ldots, n$, temos que $y_{i} y_{i+n}=\sigma_{1}\left(x_{i}\right) \sigma_{1}\left(\partial_{i}\right)=\sigma_{2}\left(x_{i} \partial_{i}\right)$ e $y_{i+n} y_{i}=\sigma_{2}\left(\partial_{i} x_{i}\right)$. Como $\partial_{i} x_{i}=x_{i} \partial_{i}+1$ e $\sigma_{2}(1)=\sigma_{1}(1) \sigma_{1}(1)=0$, temos que

$$
\sigma_{2}\left(\partial_{i} x_{i}\right)=\sigma_{2}\left(x_{i} \partial_{i}\right)
$$

Então $y_{i} y_{i+n}=y_{i+n} y_{i}$, para $i=1, \ldots, n$. Se $1 \leq i, j \leq n$, temos que $y_{i} y_{j}=\sigma_{2}\left(x_{i} x_{j}\right)=$ $\sigma_{2}\left(x_{j} x_{i}\right)=y_{j} y_{i}$. Analogamente, se $n+1 \leq i, j \leq 2 n$, temos que $y_{i} y_{j}=y_{j} y_{i}$. Agora, seja $1 \leq i \leq n$ e $n+1 \leq j \leq 2 n$, com $j \neq i+n$, ou seja, $j-n \neq i$. Então

$$
y_{i} y_{j}=\sigma_{1}\left(x_{i}\right) \sigma_{1}\left(\partial_{j-n}\right)=\sigma_{2}\left(x_{i} \partial_{j-n}\right)=\sigma_{2}\left(\partial_{j-n} x_{i}\right)=y_{j} y_{i}
$$

Concluímos que os geradores de $S_{n}$ comutam.

Seja $k\left[z_{1}, \ldots, z_{2 n}\right]$ o anel dos polinômios em $2 n$ variáveis. Os dois passos anteriores nos permite definir um homomorfismo de anéis sobrejetivo

$$
\phi: k\left[z_{1}, \ldots, z_{2 n}\right] \longrightarrow S_{n}
$$

por $\phi\left(z_{i}\right)=y_{i}$. Como os $z$ 's tem grau 1 em $k\left[z_{1}, \ldots, z_{2 n}\right]$ e os $y$ 's tem grau 1 em $S_{n}$, segue que $\phi$ é um homomorfismo de $k$-álgebras graduado.

Terceiro passo: $\phi$ é injetivo.

Seja $F\left(z_{1}, \ldots, z_{2 n}\right) \in k\left[z_{1}, \ldots, z_{2 n}\right]$. Então $F\left(z_{1}, \ldots, z_{2 n}\right)=\sum c_{\alpha \beta} z_{1}^{\alpha_{1}} \cdots z_{n}^{\alpha_{n}} z_{n+1}^{\beta_{1}} \cdots z_{2 n}^{\beta_{n}}$. Suponhamos que $\phi\left(F\left(z_{1}, \ldots, z_{2 n}\right)\right)=0$. Como $\phi$ é um homomorfismo graduado, podemos assumir que $F$ é um polinômio homogêneo. Seja

$$
F\left(y_{1}, \ldots, y_{2 n}\right)=\sum c_{\alpha \beta} y_{1}^{\alpha_{1}} \cdots y_{n}^{\alpha_{n}} y_{n+1}^{\beta_{1}} \cdots y_{2 n}^{\beta_{n}}
$$


em que $\alpha_{1}+\cdots+\alpha_{n}+\beta_{1}+\cdots+\beta_{n}=r$. Defina um operador $d \in A_{n}$ da seguinte maneira

$$
d=\sum c_{\alpha \beta} x_{1}^{\alpha_{1}} \cdots x_{n}^{\alpha_{n}} \partial_{1}^{\beta_{1}} \cdots \partial_{n}^{\beta_{n}}
$$

Então

$$
\sigma_{r}(d)=\sum c_{\alpha \beta} \sigma_{r}\left(x^{\alpha} \partial^{\beta}\right)=\sum c_{\alpha \beta} y_{1}^{\alpha_{1}} \cdots y_{n}^{\alpha_{n}} y_{n+1}^{\beta_{1}} \cdots y_{2 n}^{\beta_{n}}=F\left(y_{1}, \ldots, y_{2 n}\right)
$$

Por outro lado, $0=\phi\left(F\left(z_{1}, \ldots, z_{2 n}\right)\right)=F\left(y_{1}, \ldots, y_{2 n}\right)$. Se $\sigma_{r}(d)=\phi\left(F\left(z_{1}, \ldots, z_{2 n}\right)\right)=$ 0 , então $d \in B_{r-1}$. Assim $d$ pode ser escrito como uma combinação linear de monômios $x^{\alpha} \partial^{\beta}$ com $|\alpha|+|\beta|<r$. Por construção, $d$ é também uma combinação linear de monômios de grau $r$. Pela Proposição 1.1 .2 (Capítulo 1) a escrita de $d$ como combinação linear desses monômios é única. Logo todos os coeficientes $c_{\alpha \beta}$ são zeros. Então $F\left(z_{1}, \ldots, z_{2 n}\right)$ é o polinômio nulo e $\phi$ é injetivo como desejado, ou seja, $k\left[z_{1}, \ldots, z_{2 n}\right] \cong S_{n}=g r^{\mathcal{B}} A_{n}$.

Uma observação é que a álgebra graduada $g r^{\mathcal{C}} A_{n}$ associada à filtração por ordem também é isomorfa ao anel de polinômios em $2 n$ variáveis (veja [Cou95], Capítulo 7, Exercício 6.5).

\subsubsection{Módulos filtrados}

Para definir um módulo filtrado devemos começar com um anel filtrado. Por uma questão de simplicidade vamos dar a definição somente para a álgebra de Weyl $A_{n}$ com a filtração de Bernstein $\mathcal{B}=\left\{B_{r}\right\}_{r \in \mathbb{N}}$.

Definição 2.2.7. Seja $M$ um $A_{n}$-módulo à esquerda. Uma familia $\Gamma=\left\{\Gamma_{i}\right\}_{i \geq 0}$ de $k$-espaços vetoriais de $M$ é uma filtração do módulo $M$ se ela satisfaz:

1. $\Gamma_{0} \subseteq \Gamma_{1} \subseteq \cdots \subseteq M$

2. $\bigcup_{i \geq 0} \Gamma_{i}=M$,

3. $B_{i} \Gamma_{j} \subseteq \Gamma_{i+j}$.

Por convenção $\Gamma_{j}=\{0\}$ se $j<0$.

Embora essa seja a definição padrão de uma filtração de módulo, vamos exigir que as filtrações tenham uma condição adicional. Antes, observamos que 3. com $i=0$, implica que cada $\Gamma_{j}$ é um $k$-espaço vetorial. A quarta condição que uma filtração de módulo deve satisfazer é:

4. $\Gamma_{i}$ é um k-espaço vetorial de dimensão finita.

É claro que $\mathcal{B}$ é uma filtração de $A_{n}$ como um $A_{n}$-módulo. Um exemplo mais interessante é o $A_{n}$-módulo $k\left[x_{1}, \ldots, x_{n}\right]$. Os espaços vetoriais $\Gamma_{i}$ de todos os polinômios com grau $\leq i$ formam uma filtração de $k\left[x_{1}, \ldots, x_{n}\right]$ para a filtração de Bernstein $\mathcal{B}$. De fato as propriedades 1., 2. e 4. de uma filtração de módulo são claramente satisfeitas pelos $\Gamma_{i}$. Vejamos que 3. também é satisfeita. Sejam $P \in B_{i}$ e $f \in \Gamma_{j}$. Seja $x_{1}^{\sigma_{1}} \cdots x_{n}^{\sigma_{n}}$ um monômio com maior grau possível, de acordo com a ordem lexicográfica, dentre todos os monômios que aparecem em $f$ com coeficientes não nulos; da mesma maneira seja $x_{1}^{\alpha_{1}} \cdots x_{n}^{\alpha_{n}} \partial_{1}^{\beta_{1}} \cdots \partial_{n}^{\beta_{n}}$ um monômio com maior grau possível em $P$. Temos que $|\sigma| \leq j$ e $|\alpha|+|\beta| \leq i$. Seja $x^{\alpha} \partial^{\beta}\left(x^{\sigma}\right)$. Os 
resultados possíveis de $\partial^{\beta}\left(x^{\sigma}\right)$ são uma constante não negativa $c$ (Lema 1.1.1) ou $c x^{\gamma}$, em que $c$ é uma constante e $|\gamma|=|\sigma|-|\beta|>0$. Em todos os casos possíveis o grau de $P f$, visto como um polinômio nas variáveis $x_{1}, \ldots, x_{n}$ com coeficientes em $k$, terá no máximo grau $|\alpha|+|\gamma|=|\alpha|+|\sigma|-|\beta| \leq i-|\beta|+j-|\beta| \leq i+j$, ou seja, $\operatorname{Pf} \in \Gamma_{i+j}$.

Seguindo o padrão da Subseção 2.2.3, podemos definir o módulo graduado associado com um módulo filtrado.

Seja $M$ um $A_{n}$-módulo à esquerda e seja $\Gamma$ uma filtração de $M$ com respeito à $\mathcal{B}$. Defina a aplicação símbolo de ordem $r$ da filtração $\Gamma$ como sendo a projeção canônica

$$
\mu_{r}: \Gamma_{r} \rightarrow \Gamma_{r} / \Gamma_{r-1}
$$

Agora tome o $k$-espaço vetorial

$$
g r^{\Gamma} M=\bigoplus_{i \geq 0}\left(\Gamma_{i} / \Gamma_{i-1}\right)
$$

Definiremos uma ação de $S_{n}$ nesse $k$-espaço vetorial, em que $S_{n}=g r^{\mathcal{B}} A_{n}$. Se $a \in B_{r}$ e $u \in$ $\Gamma_{i}$, seja

$$
\sigma_{r}(a) \cdot \mu_{i}(u)=\mu_{r+i}(a u)
$$

Como os elementos de $S_{n}$ e $g r^{\Gamma} M$ são somas finitas únicas de elementos homogêneos, estendendo essa fórmula por linearidade obtemos uma ação de $S_{n}$ em $g r^{\Gamma} M$. Vamos verificar que essa ação está bem definida, isto é, não depende das escolhas de $a$ e $u$. Seja $a_{1}$ um outro elemento em $B_{r}$ tal que $\sigma_{r}\left(a_{1}\right)=\sigma_{r}(a)$. Então $\sigma_{r}\left(a_{1}-a\right)=0$ em $B_{r} / B_{r-1}$. Assim $a_{1}-a$ $\in B_{r-1}$. Como $\Gamma$ é uma filtração de $M$ com respeito à $\mathcal{B}$, temos que $\left(a_{1}-a\right) u=a_{1} u-a u$ $\in \Gamma_{r+i-1}$. Então $\mu_{r+i}\left(a_{1} u-a u\right)=0 \mathrm{em} \Gamma_{r+i} / \Gamma_{r+i-1}$. Portanto $\mu_{r+i}\left(a_{1} u\right)=\mu_{r+i}(a u) \mathrm{em}$ $\Gamma_{r+i} / \Gamma_{r+i-1}$.

Agora seja $u_{1}$ um outro elemento em $\Gamma_{i}$ tal que $\mu_{i}\left(u_{1}\right)=\mu_{i}(u)$. Como $\mu_{i}\left(u_{1}-u\right)=0$ em $\Gamma_{i} / \Gamma_{i-1}$, concluímos que $u_{1}-u \in \Gamma_{i-1}$. Novamente, como $\Gamma$ é uma filtração de $M$ com respeito à $\mathcal{B}$, temos que $a\left(u_{1}-u\right)=a u_{1}-a u \in \Gamma_{r+i-1}$. Então $\mu_{r+i}\left(a u_{1}-a u\right)=0 \mathrm{em} \Gamma_{r+i} / \Gamma_{r+i-1}$. Portanto $\mu_{r+i}\left(a u_{1}\right)=\mu_{r+i}(a u)$ em $\Gamma_{r+i} / \Gamma_{r+i-1}$. Assim a ação está bem definida.

Agora, vamos verificar que para $a \in B_{i} / B_{i-1}$ e $u \in \Gamma_{j} / \Gamma_{j-1}$ temos que $a u \in \Gamma_{i+j} / \Gamma_{i+j-1}$. Sabemos que existe $x \in B_{i}$ tal que $\sigma_{i}(x)=a$ e existe $y \in \Gamma_{j}$ tal que $\mu_{j}(y)=u$. Assim $a u=\sigma_{i}(x) \mu_{j}(y)=\mu_{i+j}(x y)$, em que $x y \in \Gamma_{i+j}$. Portanto $a u \in \Gamma_{i+j} / \Gamma_{i+j-1}$.

Portanto $\operatorname{gr}^{\Gamma} M$ é um $S_{n}$-módulo graduado com componentes homogêneas $\Gamma_{i} / \Gamma_{i-1}$. Esse $S_{n}$-módulo é chamado de módulo graduado associado à filtração $\Gamma$.

Seja $\Gamma$ a filtração de $M$ com respeito à filtração de Bernstein $\mathcal{B}$, em que $M$ é o $A_{n}$-módulo $k\left[x_{1}, \ldots, x_{n}\right]$, como definida anteriormente. Queremos determinar o módulo graduado de $M$ associado à filtração $\Gamma$. Temos que $\Gamma_{i} / \Gamma_{i-1}$ é isomorfo ao espaço vetorial de todos os polinômios homogêneos de grau $i$. Assim $g^{\Gamma} M$ é isomorfo à $k\left[x_{1}, \ldots, x_{n}\right]$ como $k$-espaços vetoriais. Entretanto vimos no Teorema 2.2.1 que $S_{n}$ é isomorfa ao anel de polinômios em $2 n$ variáveis $y_{1}, \ldots, y_{2 n}$. Queremos determinar a ação de $y_{1}, \ldots, y_{2 n}$, em um polinômio $f$ de grau $r$, o qual pode ser considerado como um elemento de $\Gamma_{r} / \Gamma_{r-1}$. Para $i=1, \ldots, n$, temos que $y_{i} \cdot f=x_{i} f$. Para $i=n+1, \ldots, 2 n$, devemos ser cuidadosos. Observamos que $y_{i} \cdot f$ é, por definição, $\mu_{r}\left(\partial_{i}(f)\right)$. Mas $\partial_{i}(f)$ é homogêneo de grau $\leq r-1$. Então $y_{i} \cdot f=0$. Em particular $\operatorname{ann}_{S_{n}}\left(g r^{\Gamma} M\right)$ é o ideal gerado por $y_{n+1}, \ldots, y_{2 n}$. 


\subsubsection{Filtrações induzidas}

Seja $M$ um $A_{n}$-módulo com uma filtração $\Gamma$ com respeito à $\mathcal{B}$. Suponha que $N$ é um submódulo de $M$. Podemos usar $\Gamma$ para construir filtrações para $N$ e $M / N$. Essas são chamadas de filtrações induzidas por $\Gamma$.

Para obter uma filtração para $N$ considere $\Gamma^{\prime}=\left\{N \cap \Gamma_{i}\right\}_{i \geq 0}$. Como $N \cap \Gamma_{i-1} \subseteq N \cap \Gamma_{i}$, para $i \geq 0$, podemos definir o quociente $N \cap \Gamma_{i} / N \cap \Gamma_{i-1}$, em que $\Gamma_{-1}=\{0\}$. A inclusão $N \hookrightarrow M$ nos permite definir aplicações lineares injetivas:

$$
\phi_{r}: N \cap \Gamma_{r} / N \cap \Gamma_{r-1} \rightarrow \Gamma_{r} / \Gamma_{r-1}
$$

pois $N \cap \Gamma_{i} \hookrightarrow M \cap \Gamma_{i}=\Gamma_{i}$ para $i \geq 0$.

Esta família de aplicações produzem uma aplicação linear:

$$
\phi: g r^{\Gamma^{\prime}} N \rightarrow g r^{\Gamma} M
$$

Um cálculo com elementos homogêneos de $S_{n}$ e de $g r^{\Gamma^{\prime}} N$ mostra que $\phi$ é um homomorfismo de $S_{n}$-módulos. Como os $\phi_{r}$ são injetivos, então $\phi$ também é. Logo $g r^{\Gamma^{\prime}} N \subseteq g r^{\Gamma} M$.

Agora vamos considerar o módulo quociente $M / N$. Seja $\Gamma_{r}^{\prime \prime}$ o subespaço de $M / N$ definido por

$$
\Gamma_{r}^{\prime \prime}=\Gamma_{r} /\left(\Gamma_{r} \cap N\right)
$$

Observamos que como $N \cap \Gamma_{r} \subseteq M \cap \Gamma_{r}=\Gamma_{r}$, podemos definir o quociente $\Gamma_{r} /\left(\Gamma_{r} \cap N\right)$. Vamos verificar que $\Gamma^{\prime \prime}=\left\{\Gamma_{i}^{\prime \prime}\right\}_{i \geq 0}$ é uma filtração de $M / N$.

Como $\Gamma_{r}$ e $N$ são subespaços vetoriais de $M$ temos que $\Gamma_{r} /\left(\Gamma_{r} \cap N\right) \cong\left(\Gamma_{r}+N\right) / N$. Como $\Gamma_{r}+N \subseteq M$, então $\left(\Gamma_{r}+N\right) / N \subseteq M / N$. Portanto $\Gamma_{i}^{\prime \prime}=\Gamma_{i} /\left(\Gamma_{i} \cap N\right) \cong\left(\Gamma_{i}+N\right) / N \subseteq M / N$, para $i \geq 0$. Além disso, $\Gamma_{i-1}+N \subseteq \Gamma_{i}+N$. Então $\left(\Gamma_{i-1}+N\right) / N \subseteq\left(\Gamma_{i}+N\right) / N$. Portanto $\Gamma_{i-1} /\left(\Gamma_{i-1} \cap N\right) \subseteq \Gamma_{i} /\left(\Gamma_{i} \cap N\right)$, ou seja, $\Gamma_{i-1}^{\prime \prime} \subseteq \Gamma_{i}^{\prime \prime}$, para $i \geq 0$. Logo vale a propriedade 1. de uma filtração de módulo. Como $\bigcup_{i \geq 0} \Gamma_{i}=M$, temos que $\bigcup_{i \geq 0} \Gamma_{i}+N=M$. Assim $\bigcup_{i \geq 0}\left(\Gamma_{i}+N\right) / N=M / N$. Portanto $\bigcup_{i \geq 0} \Gamma_{i}^{\prime \prime}=M / N$, e vale a propriedade 2. Além disso, sabemos que $\Gamma_{i}$ é um subespaço com dimensão finita, para todo $i \geq 0$. Logo $\Gamma_{i} /\left(\Gamma_{i} \cap N\right)$ tem dimensão finita, ou seja, $\Gamma_{i}^{\prime \prime}$ tem dimensão finita para todo $i \geq 0$, e vale 4 . Para mostrar a validade da propriedade 3 , seja $b \in B_{i}$ e $x \in \Gamma_{j}^{\prime \prime}$. Posso considerar que $x \in\left(\Gamma_{j}+N\right) / N$, ou seja, $x=\left(x_{1}+x_{2}\right)+N$, em que $x_{1} \in \Gamma_{j}$ e $x_{2} \in N$ (que é $A_{n}$-submódulo de $M$ ). Assim $b x=b x_{1}+b x_{2}+N \in\left(\Gamma_{i+j}+N\right) / N$, isto é, $b x \in \Gamma_{i+j} /\left(\Gamma_{i+j} \cap N\right)=\Gamma_{i+j}^{\prime \prime}$, e vale 3.

Vejamos que $\Gamma_{r}^{\prime \prime} / \Gamma_{r-1}^{\prime \prime} \cong \Gamma_{r} /\left(\Gamma_{r-1}+\Gamma_{r} \cap N\right)$.

Primeiro vamos verificar que $\Gamma_{r} \cap\left(\Gamma_{r-1}+N\right)=\Gamma_{r} \cap \Gamma_{r-1}+\Gamma_{r} \cap N$. Seja $x \in \Gamma_{r} \cap\left(\Gamma_{r-1}+N\right)$. Como $x \in \Gamma_{r-1}+N$, então $x=y+z$, em que $y \in \Gamma_{r-1}$ e $z \in N$. Como $\Gamma_{r-1} \subseteq \Gamma_{r}, y \in$ $\Gamma_{r} \cap \Gamma_{r-1} . z=x-y \in \Gamma_{r}, \operatorname{logo} z \in \Gamma_{r} \cap N$. Portanto $x=y+z \in \Gamma_{r} \cap \Gamma_{r-1}+\Gamma_{r} \cap N$. Reciprocamente, seja $x=y+z$, com $y \in \Gamma_{r} \cap \Gamma_{r-1}$ e $z \in \Gamma_{r} \cap N$. Assim $y$ e $z \in \Gamma_{r}$, ou seja, $x \in \Gamma_{r}$. Como $y \in \Gamma_{r-1}$ e $z \in N, x \in \Gamma_{r-1}+N$. Portanto $x \in \Gamma_{r} \cap\left(\Gamma_{r-1}+N\right)$. Observamos também que $\Gamma_{r-1}+\Gamma_{r} \cap N=\Gamma_{r} \cap \Gamma_{r-1}+\Gamma_{r} \cap N$. Então:

$$
\Gamma_{r} /\left(\Gamma_{r-1}+\Gamma_{r} \cap N\right)=\Gamma_{r} /\left(\Gamma_{r} \cap\left(\Gamma_{r-1}+N\right)\right) \cong\left(\Gamma_{r}+\left(\Gamma_{r-1}+N\right)\right) /\left(\Gamma_{r-1}+N\right)
$$

Assim:

$$
\Gamma_{r} /\left(\Gamma_{r-1}+\Gamma_{r} \cap N\right) \cong\left(\Gamma_{r}+N\right) /\left(\Gamma_{r-1}+N\right) \cong\left(\left(\Gamma_{r}+N\right) / N\right) /\left(\left(\Gamma_{r-1}+N\right) / N\right) \cong \Gamma_{r}^{\prime \prime} / \Gamma_{r-1}^{\prime \prime}
$$

Temos que $\Gamma_{r-1}+\Gamma_{r} \cap N \subseteq \Gamma_{r}$. Seja a aplicação linear $\varphi: \Gamma_{r} \rightarrow \Gamma_{r} /\left(\Gamma_{r-1}+\Gamma_{r} \cap N\right)$, tal 
que $x \mapsto x+\left(\Gamma_{r-1}+\Gamma_{r} \cap N\right)$. Observamos que:

$\operatorname{ker}(\varphi)=\left\{x \in \Gamma_{r} \mid x+\left(\Gamma_{r-1}+\Gamma_{r} \cap N\right)=0\right\}=\left\{x \in \Gamma_{r} \cap\left(\Gamma_{r-1}+\Gamma_{r} \cap N\right)\right\}=\Gamma_{r-1}+\Gamma_{r} \cap N$

Como $\Gamma_{r-1} \subseteq \operatorname{ker}(\varphi)$, existe a aplicação linear

$$
\pi_{r}: \Gamma_{r} / \Gamma_{r-1} \rightarrow \Gamma_{r} /\left(\Gamma_{r-1}+\Gamma_{r} \cap N\right) \cong \Gamma_{r}^{\prime \prime} / \Gamma_{r-1}^{\prime \prime}
$$

a projeção canônica, tal que $\pi_{r}\left(x+\Gamma_{r-1}\right)=\varphi(x)$. A família dessas projeções nos dá uma $k$-aplicação linear

$$
\pi: g r^{\Gamma} M \rightarrow g r^{\Gamma^{\prime \prime}} M / N
$$

Esta aplicação é um homomorfismo sobrejetivo de $S_{n}$-módulos.

Lema 2.2.1. Seja $M$ um $A_{n}$-módulo com uma filtração $\Gamma$ compativel com $\mathcal{B}$. A sequência de $S_{n}$-módulos

$$
0 \longrightarrow g r^{\Gamma^{\prime}} N \stackrel{\phi}{\rightarrow} g r^{\Gamma} M \stackrel{\pi}{\rightarrow} g r^{\Gamma^{\prime \prime}} M / N \rightarrow 0
$$

é exata.

Demonstração. Observamos que:

$$
\operatorname{ker}\left(\pi_{r}\right)=\left\{x+\Gamma_{r-1} \in \Gamma_{r} / \Gamma_{r-1} \mid \pi_{r}\left(x+\Gamma_{r-1}\right)=\varphi(x)=0\right\}=\left\{x+\Gamma_{r-1} \mid x \in \operatorname{ker}(\varphi)\right\}
$$

Então $\operatorname{ker}\left(\pi_{r}\right)=\left(\Gamma_{r-1}+\Gamma_{r} \cap N\right) / \Gamma_{r-1}$. Temos que:

$$
\left(\Gamma_{r-1}+\Gamma_{r} \cap N\right) / \Gamma_{r-1} \cong\left(\Gamma_{r} \cap N\right) /\left(\left(\Gamma_{r} \cap N\right) \cap \Gamma_{r-1}\right)=\left(\Gamma_{r} \cap N\right) /\left(\Gamma_{r-1} \cap N\right)
$$

Então temos uma sequência exata de espaços vetoriais

$$
0 \rightarrow\left(\Gamma_{r} \cap N\right) /\left(\Gamma_{r-1} \cap N\right) \stackrel{\phi_{r}}{\longrightarrow} \Gamma_{r} / \Gamma_{r-1} \stackrel{\pi_{r}}{\longrightarrow} \Gamma_{r} /\left(\Gamma_{r-1}+\Gamma_{r} \cap N\right) \rightarrow 0
$$

A sequência de $S_{n}$-módulos no Lema é obtida adicionando essas sequências de espaços vetoriais para $r \geq 0$. Portanto ela é exata.

A sequência exata do Lema 2.2.1 é muito útil. Vejamos uma aplicação típica. Seja $d \in$ $A_{n}$ de grau $r$ e seja $M=A_{n} / A_{n} \cdot d$. Tome $\mathcal{B}$ como a filtração de $A_{n}$ como $A_{n}$-módulo à esquerda. A filtração induzida em $A_{n} \cdot d$ é $\mathcal{B}_{s}^{\prime}=B_{s-r} d$. Então:

$$
\mathcal{B}_{s}^{\prime} / \mathcal{B}_{s-1}^{\prime}=B_{s-r} d / B_{s-r-1} d \cong\left(B_{s-r} / B_{s-r-1}\right) \sigma_{r}(d)
$$

Como $B_{s} / B_{s-1}$ é a componente homogênea de grau $s$ de $S_{n}$, então

$$
\operatorname{gr}^{\mathcal{B}^{\prime}}\left(A_{n} \cdot d\right) \cong S_{n} \sigma_{r}(d)
$$

Pelo Lema 2.2.1, existe uma sequência exata,

$$
0 \longrightarrow S_{n} \sigma_{r}(d) \longrightarrow S_{n} \rightarrow g r^{\mathcal{B}^{\prime}}(M) \longrightarrow 0
$$

em que $g^{\mathcal{B}^{\prime}}(M) \cong S_{n} / S_{n} \sigma_{r}(d)$. 


\subsection{Anéis e módulos Noetherianos}

Vamos provar que a $n$-ésima álgebra de Weyl $A_{n}$ é um anel Noetheriano à esquerda.

Sabemos que a imagem homomórfica de um módulo finitamente gerado é finitamente gerada. Entretanto um módulo finitamente gerado pode ter um submódulo que não é finitamente gerado. Um exemplo é o anel de polinômios em infinitas variáveis $k\left[x_{1}, x_{2}, \ldots\right]$. Tomado como módulo sobre ele mesmo este anel é um módulo à esquerda cíclico: é gerado por 1. Contudo, o ideal gerado por todas as variáveis $x_{1}, x_{2}, \ldots$ não pode ser finitamente gerado.

Um $R$-módulo à esquerda é chamado de Noetheriano se todos os seus submódulos são finitamente gerados. Espaços vetoriais de dimensão finita sobre $k$ são $k$-módulos Noetherianos. Todo ideal do anel de polinômios em uma variável $k[x]$ é um $k[x]$-módulo Noetheriano.

Existem várias definições equivalentes para $R$-módulos Noetherianos. Escolhemos a mais natural. Seguem duas mais:

Teorema 2.3.1. Seja $M$ um R-módulo à esquerda. As seguintes condições são equivalentes:

1. $M$ é Noetheriano.

2. Para cada cadeia ascendente infinita $N_{1} \subseteq N_{2} \subseteq \ldots$ de submódulos de $M$, existe $r \geq 0$ tal que $N_{i}=N_{r}$ para todo $i \geq r$.

3. Todo conjunto $\mathcal{S}$ de submódulos de $M$ contém um submódulo L que não está propriamente contido em nenhum outro submódulo em $\mathcal{S}$.

A condição 2. é conhecida como condição de cadeia ascendente. A condição 3. é a condição maximal: o submódulo L é chamado de elemento maximal de $\mathcal{S}$.

Demonstração. Suponhamos que 1 seja válida. Se $N_{1} \subseteq N_{2} \subseteq \ldots$ é uma cadeia ascendente infinita de submódulos de $M$, então $Q=\bigcup_{i \geq 1} N_{i}$ é um submódulo de $M$. Logo $Q$ é gerado por uma quantidade finita de elementos, $u_{1}, \ldots, u_{t}$. Assim existe $r \geq 0$, tal que $u_{1}, \ldots, u_{t} \in$ $N_{r}$. Então $Q=N_{r}=N_{i}$, para todo $i \geq r$, como desejado em 2 .

Vamos assumir que 2 é válida. Vamos provar 3. por contradição. Suponhamos que $\mathcal{S}$ não contém um elemento maximal. Se $N_{1} \subseteq N_{2} \subseteq \ldots \subseteq N_{r}$ é alguma cadeia de elementos de $\mathcal{S}$, então podemos torná-la maior. Como nenhum elemento de $\mathcal{S}$ é maximal, existe $N_{r+1} \in \mathcal{S}$ tal que $N_{r} \subsetneq N_{r+1}$. Neste caso podemos construir uma cadeia ascendente infinita própria de submódulos de $M$, contradizendo 2 .

Finalmente, vamos assumir 3. Sejam $N$ um submódulo de $M, \mathcal{S}$ o conjunto de todos os submódulos de $N$ finitamente gerados e $L$ o elemento maximal de $\mathcal{S}$. Suponhamos que $L \subsetneq N$ e tomemos $u \in N \backslash L$. Então $L+R u$ é finitamente gerado (pois $L$ é) e contém $L$ propriamente: uma contradição, pois $L$ é maximal em $\mathcal{S}$. Então $L=N$, ou seja, $N$ é finitamente gerado. Portanto $M$ é Noetheriano.

Vamos enunciar algumas propriedades básicas dos módulos Noetherianos, mas antes vamos mostrar um lema técnico.

Lema 2.3.1. Seja $M$ um módulo à esquerda sobre um anel $R$. Sejam $N, P_{1}$ e $P_{2}$ submódulos de $M$ tal que $P_{2} \subseteq P_{1}$. Se $N+P_{1}=N+P_{2}$ e $N \cap P_{1}=N \cap P_{2}$, então $P_{1}=P_{2}$.

Demonstração. Precisamos somente mostrar que $P_{1} \subseteq P_{2}$. Suponhamos que $u_{1} \in P_{1}$. Mas $P_{1} \subseteq N+P_{1}$, logo $u_{1} \in N+P_{1}=N+P_{2}$. Então $u_{1}=x+u_{2}$, com $x \in N, u_{2} \in P_{2}$. Assim $x=u_{1}-u_{2} \in N \cap P_{1}=N \cap P_{2}$. Em particular, $x \in P_{2}$. Portanto $u_{1}=x+u_{2} \in P_{2}$, como desejado. 
Proposição 2.3.1. Sejam $M$ um módulo à esquerda sobre $R$ e $N$ um submódulo de $M$.

1. $M$ é Noetheriano se, e somente se, $M / N$ e $N$ são Noetherianos.

2. Seja $N^{\prime}$ um outro submódulo de $M$ e suponha que $M=N+N^{\prime}$. Se $N, N^{\prime}$ são Noetherianos, então $M$ é Noetheriano.

Demonstração. 1. É claro que um submódulo de um módulo Noetheriano é Noetheriano. Logo, se $M$ é Noetheriano então $N$ é Noetheriano. Por outro lado, um submódulo de $M / N$ é da forma $L / N$ para algum submódulo $L$ de $M$ que contém $N$ (Teorema da Correspondência entre $R$-módulos). Se $M$ é Noetheriano então $L$ é finitamente gerado. Logo $L / N$ é finitamente gerado. Assim $M / N$ é Noetheriano.

Reciprocamente, suponhamos que $N$ e $M / N$ são Noetherianos. Seja $L_{1} \subseteq L_{2} \subseteq \ldots$, uma cadeia ascendente infinita de submódulos de $M$. Então $\left(L_{1} \cap N\right) \subseteq\left(L_{2} \cap N\right) \subseteq \ldots$, é uma cadeia ascendente de submódulos de $N$. Como $N$ é Noetheriano, esta cadeia estaciona. Em outras palavras, existe $s$ tal que $\left(L_{s} \cap N\right)=\left(L_{i} \cap N\right)$, para todo $i \geq s$. Similarmente, a cadeia $\left(N+L_{1}\right) / N \subseteq\left(N+L_{2}\right) / N \subseteq \ldots$, de submódulos de $M / N$, também estaciona. Logo existe $r$ tal que $N+L_{r}=N+L_{i}$, para todo $i \geq r$. Tomando $t=\max \{s, r\}$, temos que $N+L_{t}=N+L_{i}$ e $L_{t} \cap N=L_{i} \cap N$, para $i \geq t$. Então pelo Lema 2.3.1, $L_{t}=L_{i}$, para $i \geq t$. Portanto $M$ é Noetheriano.

2. Observamos que $M / N=\left(N+N^{\prime}\right) / N \cong N^{\prime} /\left(N^{\prime} \cap N\right)$. Como $N^{\prime}$ é Noetheriano e $N^{\prime} \cap N$ é submódulo de $N^{\prime}$, temos que $M / N$ é Noetheriano, por 1. Como $N$ e $M / N$ são Noetherianos, usando 1 novamente concluimos que $M$ é Noetheriano.

\subsubsection{Anéis Noetherianos}

Dizemos que um anel $R$ é um anel Noetheriano à esquerda se $R$ é Noetheriano como um $R$-módulo à esquerda. Corpos e domínios de ideias principais, como $\mathbb{Z}$ ou $k[x]$, são exemplos de anéis Noetherianos à esquerda.

Os anéis mais importantes em geometria algébrica são Noetherianos, pois o anel de polinômios em uma quantidade finita de variáveis é Noetheriano. Isto foi provado por D. Hilbert em 1890 e é peça fundamental da álgebra comutativa. Vamos apresentar uma versão mais moderna do resultado de Hilbert, mas o teorema original segue como consequência.

Teorema 2.3.2 (Teorema da Base de Hilbert). Seja $R$ um anel comutativo Noetheriano. $O$ anel de polinômios $R[x]$ é Noetheriano.

Demonstração. Suponha que $R[x]$ não é Noetheriano. Seja $I$ um ideal de $R[x]$ que não é finitamente gerado. Vamos construir, indutivamente, uma cadeia ascendente infinita de ideais em $R$; assim conseguiremos uma contradição. Vamos escolher $f_{1} \in I$ com o menor grau possível, de acordo com a ordem lexicográfica. Como $I$ não é finitamente gerado, então $I \neq\left\langle f_{1}\right\rangle$. Tome $f_{2} \in I \backslash\left\langle f_{1}\right\rangle$ de grau minimal. Para cada $r$ tendo encontrado, $f_{1}, \ldots, f_{r}$ $\in I$, seja $f_{r+1}$ o polinômio com o menor grau possível em $I \backslash\left\langle f_{1}, \ldots, f_{r}\right\rangle$. Como $I$ não é finitamente gerado, essa construção produz uma sequência infinita de polinômios $f_{1}, f_{2}, \ldots$ $\in I$. Seja $n_{i}$ o grau e $a_{i}$ o coeficiente líder de $f_{i}$.

Como $R$ é Noetheriano, a cadeia ascendente de ideais $\left\langle a_{1}\right\rangle \subseteq\left\langle a_{1}, a_{2}\right\rangle \subseteq \ldots$, deve estacionar. Para algum $r,\left\langle a_{1}, \ldots, a_{r}\right\rangle=\left\langle a_{1}, \ldots, a_{r+1}\right\rangle$. Então $a_{r+1}=\sum_{i=1}^{r} b_{i} a_{i}$, para alguns $b_{i} \in$ R. Seja

$$
g=f_{r+1}-\sum_{i=1}^{r} b_{i} x^{n_{r+1}-n_{i}} f_{i}
$$


Por construção os graus satisfazem $n_{1} \leq n_{2} \leq \ldots$, logo $g$ é de fato um polinômio. Observamos que como $f_{1}, \ldots, f_{r}, f_{r+1} \in I$, que é um ideal de $R[x]$, então $g \in I$. Além disso, como $f_{r+1} \notin\left\langle f_{1}, \ldots, f_{r}\right\rangle$, temos que $g \notin\left\langle f_{1}, \ldots, f_{r}\right\rangle$. Agora vamos analisar o grau de $g$; primeiro observamos que $f_{r+1}-a_{r+1} x^{n_{r+1}}$ tem grau menor do que o grau de $f_{r+1}$. Temos que

$$
g=f_{r+1}-\sum_{i=1}^{r} b_{i} x^{n_{r+1}-n_{i}} f_{i}=f_{r+1}-\sum_{i=1}^{r} b_{i} x^{n_{r+1}-n_{i}}\left(a_{i} x^{n_{i}}+f_{i}^{\prime}\right)
$$

em que cada $f_{i}^{\prime}$ tem grau menor que $n_{i}$. Assim

$$
g=f_{r+1}-\sum_{i=1}^{r} a_{i} b_{i} x^{n_{r+1}}-\sum_{i=1}^{r} b_{i} x^{n_{r+1}-n_{i}} f_{i}^{\prime}=f_{r+1}-a_{r+1} x^{n_{r+1}}-\sum_{i=1}^{r} b_{i} x^{n_{r+1}-n_{i}} f_{i}^{\prime}
$$

Portanto, $g$ tem grau menor do que o grau de $f_{r+1}$, uma contradição. Portanto $I$ deve ser finitamente gerado.

Uma indução simples usando o Teorema 2.3.2 é tudo o que precisamos para provar o Teorema da Base de Hilbert original:

Corolário 2.3.1. Seja $k$ um corpo. O anel de polinômios $k\left[x_{1}, \ldots, x_{n}\right]$ é Noetheriano.

Embora todo ideal de $k\left[x_{1}, \ldots, x_{n}\right]$ seja finitamente gerado, não é verdade que existe um limite superior para a quantidade de geradores dos ideais neste anel. Por exemplo, é fácil de construir ideais $I_{r}$ de $k\left[x_{1}, x_{2}\right]$ que não podem ser gerados por menos do que $r$ elementos (veja [Cou95], Capítulo 8, Exercício 4.2).

Vamos usar o Teorema da Base de Hilbert para provar que $A_{n}$ é um anel Noetheriano à esquerda. Na verdade, isso seguirá de um teorema um pouco mais geral. Recordamos que $\mathcal{B}$ é a filtração de Bernstein de $A_{n}$ e $S_{n}=g r^{\mathcal{B}} A_{n}$ é a álgebra graduada de $A_{n}$ associada a filtração $\mathcal{B}$.

Teorema 2.3.3. Seja $M$ um $A_{n}$-módulo à esquerda com uma filtração $\Gamma$ com respeito à filtração de Bernstein $\mathcal{B}$. Se $\mathrm{gr}^{\Gamma} M$ é um $S_{n}$-módulo Noetheriano, então $M$ é Noetheriano.

Demonstração. Sejam $N$ um submódulo de $M$, e $\Gamma^{\prime}$ a filtração de $N$ induzida por $\Gamma$. Como $g r^{\Gamma^{\prime}} N \subseteq g r^{\Gamma} M$ e o último é Noetheriano, concluímos que $g r^{\Gamma^{\prime}} N$ é finitamente gerado.

Como os geradores de $g^{\Gamma^{\prime}} N$ são uma quantidade finita, eles tem grau $\leq m$, para algum inteiro $m$. Desejamos mostrar que $N$ é gerado por elementos em $\Gamma_{m}^{\prime}$. Suponhamos que ele não é, e seja $r$ o menor inteiro para o qual existe $v \in \Gamma_{r}^{\prime}$ que não pode ser gerado por elementos de $\Gamma_{m}^{\prime}$. Claramente $r>m$. Seja $\mu_{r}$ a aplicação símbolo de ordem $r$ de $\Gamma^{\prime}$, ou seja, $\mu_{r}: \Gamma_{r}^{\prime} \rightarrow \Gamma_{r}^{\prime} / \Gamma_{r-1}^{\prime}$. Como $g r^{\Gamma^{\prime}} N$ é finitamente gerado e $\mu_{r}(v) \in \Gamma_{r}^{\prime} / \Gamma_{r-1}^{\prime}$, existem $a_{i} \in A_{n}$ e $u_{i} \in \Gamma_{r_{i}}^{\prime}$, tal que

$$
\mu_{r}(v)=\sum_{i=1}^{s} \sigma_{r-r_{i}}\left(a_{i}\right) \mu_{r_{i}}\left(u_{i}\right)
$$

em que $r_{i} \leq m$, para $i=1,2, \ldots, s$. Então $\mu_{r}\left(v-\sum_{i=1}^{s} a_{i} u_{i}\right)=0$, ou seja,

$$
v-\sum_{i=1}^{s} a_{i} u_{i} \in \Gamma_{r-1}^{\prime}
$$

que por conta da minimalidade de $r$, pode ser escrito como uma $A_{n}$-combinação linear de elementos em $\Gamma_{m}^{\prime}$. Então $v$ pode ser escrito como uma $A_{n}$-combinação linear de elementos em $\Gamma_{m}^{\prime}$. Isso é uma contradição. Logo $N$ pode ser gerado por elementos em $\Gamma_{m}^{\prime}$. 
Para terminar a prova devemos mostrar que uma quantidade finita de elementos de $\Gamma_{m}^{\prime}$ são suficientes para gerar $N$. Mas $\Gamma_{m}^{\prime}$ é um subespaço do espaço vetorial $\Gamma_{m}$ de dimensão finita. Então ele tem uma base finita, que será um conjunto de geradores para $N$ como $A_{n}$-módulo.

Corolário 2.3.2. $A_{n}$ é um anel Noetheriano à esquerda.

Demonstração. O anel graduado $S_{n}=g r^{\mathcal{B}} A_{n}$ associado à filtração de Bernstein, é um anel de polinômios em $2 n$ variáveis pelo Teorema 2.2.1. Então pelo Corolário 2.3.1 do Teorema da Base de Hilbert, $S_{n}$ é Noetheriano. Portanto, pelo Teorema 2.3.3, $A_{n}$ é Noetheriano à esquerda como $A_{n}$-módulo. Concluímos assim que $A_{n}$ é um anel Noetheriano à esquerda.

\subsection{Módulos de peso sobre $\boldsymbol{A}=\boldsymbol{A}_{k, \mathrm{I}}$}

Esta seção será baseada em [FGM14][Seção 3.1].

Seja $A=A_{k, \mathrm{I}}$ a álgebra de Weyl de posto infinito, cf. Capítulo 1, Seção 1.3. Vamos considerar que $k$ é um corpo que tem característica zero e é algebricamente fechado; como $k \subseteq A$ então um $A$-módulo $M$ é um $k$-espaço vetorial.

Definição 2.4.1. Seja $M$ um A-módulo e $\mathfrak{m}$ um ideal maximal em $A_{0}$. Um elemento $x \in$ $M$ é chamado de peso se $\mathfrak{m} \cdot x=\{0\}$. O A-módulo $M$ é chamado de módulo de peso se ele tem uma base consistindo de elementos de peso.

Devemos lembrar que pelo Lema 1.3.1, do Capítulo 1, todo ideal maximal em $A_{0}$, tem a forma $\mathfrak{m}_{\mathbf{p}}=\left\langle t_{i}-p_{i}\right\rangle_{i \in \mathrm{I}}$, para algum $\mathbf{p}=\left(p_{i}\right)_{i \in \mathrm{I}} \in k^{\mathrm{I}}$, em que $t_{i}=X_{i} Y_{i}, i \in \mathrm{I}$.

Definição 2.4.2. Para $\mathbf{p} \in k^{\mathrm{I}}, M_{\mathbf{p}}=\left\{x \in M \mid t_{i} \cdot x=p_{i} \cdot x, \forall i \in \mathrm{I}\right\}$ é o espaço de peso com peso $\mathbf{p}$.

A dimensão de $M_{\mathbf{p}}$ como $k$-espaço vetorial é chamada de multiplicidade do peso de $M_{\mathbf{p}}$.

Suponhamos que $x \in M$ seja um elemento de peso tal que $x \in M_{\mathbf{p}}$, para $\mathbf{p} \in k^{\mathrm{I}}$. Vamos analisar o que acontece quando $A$ age em $x$. Podemos analisar utilizando os geradores de $A$.

1. Seja $X_{j}$, com $j \in \mathrm{I}$.

(a) Quando $i \neq j$, temos que:

$$
t_{i} X_{j} \cdot x=X_{i} Y_{i} X_{j} \cdot x=X_{j} X_{i} Y_{i} \cdot x=X_{j} t_{i} \cdot x=X_{j} p_{i} \cdot x=p_{i} X_{j} \cdot x
$$

Então $t_{i} X_{j} \cdot x=p_{i} X_{j} \cdot x$.

(b) Quando $i=j$, temos que:

$$
t_{i} X_{i} \cdot x=X_{i} Y_{i} X_{i} \cdot x=X_{i} X_{i} Y_{i} \cdot x+X_{i} \cdot x=X_{i} t_{i} \cdot x+X_{i} \cdot x=\left(p_{i}+1\right) X_{i} \cdot x
$$

Então $t_{i} X_{i} \cdot x=\left(p_{i}+1\right) X_{i} \cdot x$

Portanto $X_{j} \cdot x \in M_{\mathbf{p}+\mathbf{e}_{j}}$, em que $\mathbf{e}_{j}$ tem 1 na $j$-ésima coordenada e 0 nas outras.

2. Seja $Y_{j}, \operatorname{com} j \in \mathrm{I}$.

(a) Quando $i \neq j$, temos que:

$$
t_{i} Y_{j} \cdot x=X_{i} Y_{i} Y_{j} \cdot x=Y_{j} X_{i} Y_{i} \cdot x=Y_{j} t_{i} \cdot x=Y_{j} p_{i} \cdot x=p_{i} Y_{j} \cdot x
$$

Então $t_{i} Y_{j} \cdot x=p_{i} Y_{j} \cdot x$. 
(b) Quando $i=j$, temos que:

$$
\begin{aligned}
& t_{i} Y_{i} \cdot x=X_{i} Y_{i} Y_{i} \cdot x=Y_{i} X_{i} Y_{i} \cdot x-Y_{i} \cdot x=Y_{i} t_{i} \cdot x-Y_{i} \cdot x=p_{i} Y_{i} \cdot x-Y_{i} \cdot x=\left(p_{i}-1\right) Y_{i} \cdot x \\
& \text { Então } t_{i} Y_{i} \cdot x=\left(p_{i}-1\right) Y_{i} \cdot x
\end{aligned}
$$

Portanto $Y_{j} \cdot x \in M_{\mathbf{p}-\mathbf{e}_{j}}$, em que $\mathbf{e}_{j}$ tem 1 na $j$-ésima coordenada e 0 nas outras.

Concluímos que se $x \in M_{\mathbf{p}}$ então $A \cdot x \subseteq \sum_{\mathbf{v} \in \mathbb{Z}_{f}^{\mathrm{I}}} M_{\mathbf{p}+\mathbf{v}}$.

Vamos descobrir a estrutura de um $A$-módulo de peso.

Seja $M$ um $A$-módulo de peso. Pela Definição 2.4.1, ele possui uma base consistindo de elementos de peso, ou seja, $\left\{m_{j}\right\}_{j \in J}$ é uma base de $M, J$ é um conjunto de índices, tal que cada $m_{j}$ é um elemento de peso. Temos que $M=\bigoplus_{j \in J} A \cdot m_{j}$, em que $A \cdot m_{j}$ é o $A$-submódulo de $M$ gerado por $m_{j}$; mas cada $m_{j}$ é um elemento de peso e já sabemos como é a ação de $A$ em um elemento de peso. Portanto dizer que $M$ é um $A$-módulo de peso é o mesmo que dizer que $M$ tem a seguinte estrutura:

$$
M=\bigoplus_{\mathbf{p} \in k^{\mathrm{I}}} M_{\mathbf{p}}
$$

Definição 2.4.3. Seja $M$ um A-módulo de peso. O conjunto de todos $\mathbf{p} \in k^{\mathrm{I}}$ tal que $M_{\mathbf{p}} \neq$ $\{0\}$ é chamado de suporte de $M$ e é denotado por supp $(M)$.

Observamos que se $M$ é um $A$-módulo de peso tal que $\mathbf{p} \in \operatorname{supp}(M)$, o $A$-submódulo gerado por $M_{\mathbf{p}}$, isto é, $A \cdot M_{\mathbf{p}}$ tem suporte contido em $\mathbf{p}+\mathbb{Z}_{f}^{\mathrm{I}}$.

Agora vamos analisar a categoria que tem como objetos os $A$-módulos de peso.

Sejam $A$-mod a categoria de todos os $A$-módulos e $\mathfrak{W}$ a subcategoria de $A$-mod consistindo de todos os $A$-módulos de peso. Observamos que $\mathfrak{W}$ é uma subcategoria plena de $A$-mod, pois um homomorfismo entre dois $A$-módulos de peso, é simplesmente um homomorfismo entre dois $A$-módulos. Então para $M, N \in \mathfrak{W}$ temos que $\operatorname{Hom}_{\mathfrak{W}}(M, N)=$ $\operatorname{Hom}_{A}(M, N)$.

Para $\mathbf{p} \in k^{\mathrm{I}}$, denotamos por $\mathfrak{W}_{\mathbf{p}}$ a subcategoria plena de $\mathfrak{W}$ consistindo de todos os $A$-módulos de peso $M$ tais que $\operatorname{supp}(M) \subseteq \mathbf{p}+\mathbb{Z}_{f}^{\mathrm{I}}$.

Proposição 2.4.1. $\mathfrak{W}_{\mathbf{p}}=\mathfrak{W}_{\mathbf{m}}$ se, e somente se, $\mathbf{m} \in \mathbf{p}+\mathbb{Z}_{f}^{\mathrm{I}}$.

Demonstração. Suponhamos que $\mathfrak{W}_{\mathbf{p}}=\mathfrak{W}_{\mathbf{m}}$ e seja $M \in \mathfrak{W}_{\mathbf{p}}=\mathfrak{W}_{\mathbf{m}}$. Então $\operatorname{supp}(M) \subseteq$ $\mathbf{m}+\mathbb{Z}_{f}^{\mathrm{I}}$ e $\operatorname{supp}(M) \subseteq \mathbf{p}+\mathbb{Z}_{f}^{\mathrm{I}}$. Assim $\operatorname{supp}(M)=\left\{\mathbf{q} \mid \mathbf{q}=\mathbf{m}+\mathbf{z}, \mathbf{z} \in \mathbb{Z}_{f}^{\mathrm{I}}\right\}$ e $\operatorname{supp}(M)=$ $\left\{\mathbf{q} \mid \mathbf{q}=\mathbf{p}+\mathbf{z}, \mathbf{z} \in \mathbb{Z}_{f}^{\mathrm{I}}\right\}$. Seja $\mathbf{q}^{\prime} \in \operatorname{supp}(M)$, então $\mathbf{q}^{\prime}=\mathbf{p}+\mathbf{z}_{1}=\mathbf{m}+\mathbf{z}_{2}, \operatorname{com} \mathbf{z}_{1}, \mathbf{z}_{2} \in \mathbb{Z}_{f}^{\mathrm{I}}$. Portanto $\mathbf{m}=\mathbf{p}+\left(\mathbf{z}_{1}-\mathbf{z}_{2}\right) \in \mathbf{p}+\mathbb{Z}_{f}^{\mathrm{I}}$.

Reciprocamente, seja $\mathbf{m} \in \mathbf{p}+\mathbb{Z}_{f}^{\mathrm{I}}$. Então existe $\mathbf{z} \in \mathbb{Z}_{f}^{\mathrm{I}}$ tal que $\mathbf{m}=\mathbf{p}+\mathbf{z}$. Seja $M$ $\in \mathfrak{W}_{\mathbf{m}}$. Logo $\operatorname{supp}(M) \subseteq \mathbf{m}+\mathbb{Z}_{f}^{\mathrm{I}}=\mathbf{p}+\mathbf{z}+\mathbb{Z}_{f}^{\mathrm{I}}=\mathbf{p}+\mathbb{Z}_{f}^{\mathrm{I}}$. Assim $M \in \mathfrak{W}_{\mathbf{p}}$. De maneira análoga, se $N \in \mathfrak{W}_{\mathbf{p}}$, $\operatorname{supp}(N) \subseteq \mathbf{p}+\mathbb{Z}_{f}^{\mathrm{I}}=\mathbf{m}-\mathbf{z}+\mathbb{Z}_{f}^{\mathrm{I}}=\mathbf{m}+\mathbb{Z}_{f}^{\mathrm{I}}$. Assim $N \in \mathfrak{W}_{\mathbf{m}}$. Portanto $\mathfrak{W}_{\mathbf{p}}=\mathfrak{W}_{\mathbf{m}}$. $k^{\mathrm{I}}$.

Vamos entender a relação entre a categoria $\mathfrak{W}$ e suas subcategorias plenas $\mathfrak{W}_{\mathbf{p}}, \operatorname{com} \mathbf{p} \in$

Dado $M \in \mathfrak{W}$ podemos escrevê-lo na forma:

$$
M=\sum_{\mathbf{v} \in \mathbb{Z}_{f}^{\mathrm{I}}} M_{\mathbf{p}_{1}+\mathbf{v}} \bigoplus \sum_{\mathbf{v} \in \mathbb{Z}_{f}^{\mathrm{I}}} M_{\mathbf{p}_{2}+\mathbf{v}} \bigoplus \ldots
$$


em que $\mathbf{p}_{i}-\mathbf{p}_{j} \notin \mathbb{Z}_{f}^{\mathrm{I}}$, tomados dois a dois. Para cada $\xi \in k^{\mathrm{I}} / \mathbb{Z}_{f}^{\mathrm{I}}$, seja,

$$
M(\xi)=\bigoplus_{\mathbf{p}_{\xi} \in \xi} M_{\mathbf{p}_{\xi}}
$$

em que $\mathbf{p}_{\xi}$ é um representante da classe $\xi$. Temos que $M(\xi)$ é $A$-submódulo de $M$ visto que $A \cdot M_{\mathbf{p}_{\xi}} \subseteq \sum_{\mathbf{v} \in \mathbb{Z}_{f}^{\mathrm{I}}} M_{\mathbf{p}_{\xi}+\mathbf{v}} \cdot$

Logo $M=\bigoplus_{\xi} M(\xi)$. Então $M \in \mathfrak{W}_{\mathbf{p}_{\xi_{1}}} \bigoplus \mathfrak{W}_{\mathbf{p}_{\xi_{2}}} \bigoplus \ldots$, ou seja, temos a decomposição usual

$$
\mathfrak{W} \cong \bigoplus_{\xi \in k^{\mathrm{I}} / \mathbb{Z}_{f}^{\mathrm{I}}} \mathfrak{W}_{\mathbf{p}_{\xi}}
$$

em que $\mathbf{p}_{\xi} \in \xi$ é algum representante fixo da classe $\xi$ em $k^{\mathrm{I}} / \mathbb{Z}_{f}^{\mathrm{I}}$.

Considerando uma álgebra de Weyl generalizada cf. Capítulo 1, Seção 1.4, existe a definição de um módulo de peso sobre essa álgebra, que apresentaremos no Capítulo 3. Um caso particular aparece em [BB00] em que a álgebra de Weyl generalizada é $\mathcal{A}=\bigotimes_{i=1}^{n} \mathcal{A}_{i}$, em que $\mathcal{A}_{i}=k\left[H_{i}\right]\left(\sigma_{i}, a_{i}\right)$, como definida no Capítulo 1 . Um $\mathcal{A}$-módulo $M$ é chamado de módulo de peso generalizado (respec. de peso) se $M=\bigoplus_{\lambda \in k^{n}} M^{\lambda}$ (respec. se $M=\bigoplus_{\lambda \in k^{n}} M_{\lambda}$ ), em que o espaço vetorial de dimensão finita $M^{\lambda}$ (respec. $M_{\lambda}$ ) consiste de elementos $m \in M$ tais que $\left(H_{i}-\lambda_{i}\right)^{r} m=0$ (respec. $\left(H_{i}-\lambda_{i}\right) m=0$ ) para algum $r=r(m)$.

\subsection{Módulos de peso projetivos sobre $\boldsymbol{A}=\boldsymbol{A}_{k, \mathrm{I}}$}

Basearemos grande parte desta seção em [FGM14][Seção 3.2].

Seja $A_{0}$ a subálgebra comutativa maximal de $A=A_{k, \mathrm{I}}$, cf. Capítulo 1, Seção 1.3.

Vamos considerar agora $A_{0}$-mod, a categoria de todos os $A_{0}$-módulos.

Observação 2.5.1. Seja $M$ um módulo à esquerda sobre um anel comutativo $R$. Então podemos considerar $M$ como $R$-módulo à direita, com $m r:=r m$, para todos $r \in R, m \in M$. Similarmente, qualquer $R$-módulo à direita pode ser considerado como $R$-módulo à esquerda. Por esta razão, para anéis comutativos não precisamos fazer distinção entre $R$-módulos à esquerda e à direita. Simplesmente chamamos eles de $R$-módulos. Como $A_{0}$ é comutativa, todo $A_{0}$-módulo à esquerda é também $A_{0}$-módulo à direita, e vice versa.

Para $\mathbf{p} \in k^{\mathrm{I}}$, denotamos por $\mathfrak{V}_{\mathbf{p}}$ a subcategoria plena de $A_{0}$-mod consistindo de todos $A_{0}$-módulos $N$ tais que $\mathfrak{m}_{\mathbf{p}} \cdot N=\{0\}$.

Inicialmente notamos que $A_{0} / \mathfrak{m}_{\mathbf{p}}$ é um $A_{0}$-módulo irredutível e pertence a $\mathfrak{V}_{\mathbf{p}}$. Também vamos denotá-lo por:

$$
k_{\mathbf{p}}:=A_{0} / \mathfrak{m}_{\mathbf{p}}
$$

Observamos que para $M, N \in \mathfrak{V}_{\mathbf{p}}$ temos que $\operatorname{Hom}_{\mathfrak{V}_{\mathbf{p}}}(M, N)=\operatorname{Hom}_{A_{0}}(M, N)$.

Pela Definição 2.1.2, $N \in \mathfrak{V}_{\mathbf{p}}$ é um $A_{0}$-módulo de torção, em que $\operatorname{ann}_{A_{0}}(N)=\mathfrak{m}_{\mathbf{p}}$, pois $\mathfrak{m}_{\mathbf{p}}$ é um ideal maximal em $A_{0}$ (observamos que $A_{0}$ é um domínio de integridade). Suponhamos que $\{0\} \neq N \in \mathfrak{V}_{\mathbf{p}}$ seja irredutível. Então, pelo Lema 2.1.1(1), temos que

$$
N \cong A_{0} / \mathfrak{m}_{\mathbf{p}}
$$

A categoria $\mathfrak{V}_{\mathbf{p}}$ não tem auto extensões, então qualquer $N \in \mathfrak{V}_{\mathbf{p}}$, que não seja irredutível, é uma soma direta de $A_{0}$-módulos $A_{0} / \mathfrak{m}_{\mathbf{p}}$.

Vamos verificar que $A_{0} / \mathfrak{m}_{\mathbf{p}}$ é um $A_{0}$-módulo projetivo em $\mathfrak{V}_{\mathbf{p}}$. Notemos que $A_{0} / \mathfrak{m}_{\mathbf{p}}$ é finitamente gerado, como $A_{0}$-módulo, por $\left\{1+\mathfrak{m}_{\mathbf{p}}\right\}=1_{k_{\mathbf{p}}}$. 
Para todos $M, N \in \mathfrak{V}_{\mathrm{p}}$, tais que existe um epimorfismo $g: M \rightarrow N$ e para todo

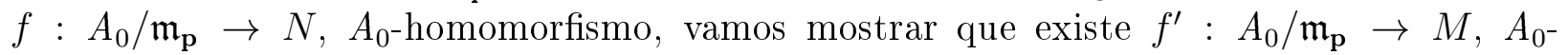
homomorfismo, tal que $g f^{\prime}=f$, ou seja, vamos mostrar que o seguinte diagrama comuta:

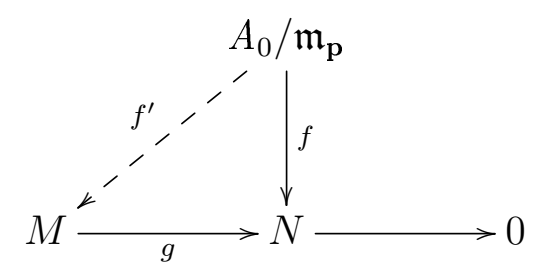

Observamos primeiramente que $\mathfrak{m}_{\mathbf{p}} \cdot M=\mathfrak{m}_{\mathbf{p}} \cdot N=\{0\}$.

Seja $f\left(1_{k_{\mathbf{p}}}\right)=n_{i}$, em que $n_{i} \in N$. Sabemos que $\mathfrak{m}_{\mathbf{p}} \cdot 1_{k_{\mathbf{p}}}=\{\overline{0}\}$. Temos que $f(\overline{0})=$ $f\left(\mathfrak{m}_{\mathbf{p}} \cdot 1_{k_{\mathbf{p}}}\right)=\mathfrak{m}_{\mathbf{p}} \cdot f\left(1_{k_{\mathbf{p}}}\right)=\mathfrak{m}_{\mathbf{p}} \cdot n_{i}=\{0\}$, pois $N \in \mathfrak{V}_{\mathbf{p}}$. Então se $\bar{a}=\bar{b}$ em $A_{0} / \mathfrak{m}_{\mathbf{p}}$, temos que $f(\bar{a}-\bar{b})=f(\overline{0})=0$, isto é, $f(\bar{a})=f(\bar{b})$. Portanto $f$ está bem definida.

Como $g$ é epimorfismo, existe $m_{i} \in M$ tal que $g\left(m_{i}\right)=n_{i}$. Novamente, $\mathfrak{m}_{\mathbf{p}} \cdot m_{i}=\{0\}$, pois $M \in \mathfrak{V}_{\mathbf{p}}$. Assim $g(0)=g\left(\mathfrak{m}_{\mathbf{p}} \cdot m_{i}\right)=\mathfrak{m}_{\mathbf{p}} \cdot g\left(m_{i}\right)=\mathfrak{m}_{\mathbf{p}} \cdot n_{i}=\{0\}$. Então se $m_{i}=m_{j}$ em $M$, temos que $g\left(m_{j}-m_{i}\right)=g(0)=0$, isto é, $g\left(m_{j}\right)=g\left(m_{i}\right)=n_{i}$. Portanto $g$ está bem definida.

Definimos $f^{\prime}: A_{0} / \mathfrak{m}_{\mathbf{p}} \rightarrow M$, por $f^{\prime}\left(1_{k_{\mathbf{p}}}\right)=m_{i}$. Temos que $f^{\prime}(\overline{0})=f^{\prime}\left(\mathfrak{m}_{\mathbf{p}} \cdot 1_{k_{\mathbf{p}}}\right)=$ $\mathfrak{m}_{\mathbf{p}} \cdot f^{\prime}\left(1_{k_{\mathbf{p}}}\right)=\mathfrak{m}_{\mathbf{p}} \cdot m_{i}=\{0\}$. Se $\bar{a}=\bar{b}$ em $A_{0} / \mathfrak{m}_{\mathbf{p}}$, temos que $f^{\prime}(\bar{a})=f^{\prime}(\bar{b})$. Portanto $f^{\prime}$ também está bem definida.

Então $\left(g f^{\prime}\right)\left(1_{k_{\mathbf{p}}}\right)=g\left(f^{\prime}\left(1_{k_{\mathbf{p}}}\right)=g\left(m_{i}\right)=n_{i}=f\left(1_{k_{\mathbf{p}}}\right)\right.$. Estendendo por linearidade, temos que $g f^{\prime}=f$. Portanto $A_{0} / \mathfrak{m}_{\mathbf{p}}$ é um $A_{0}$-módulo projetivo em $\mathfrak{V}_{\mathbf{p}}$.

Concluímos que a categoria $\mathfrak{V}_{\mathrm{p}}$, tem um único objeto simples (irredutível)

$$
k_{\mathbf{p}}:=A_{0} / \mathfrak{m}_{\mathbf{p}}
$$

a menos de isomorfismo, que é também projetivo.

Definição 2.5.1. Seja $\mathcal{C}$ uma categoria abeliana. $\mathcal{C}$ é uma categoria semissimples se existe uma coleção de objetos simples $\left\{X_{\alpha}\right\} \subseteq \mathcal{C}$, tal que qualquer objeto $X \in \mathcal{C}$, é uma soma direta dessa coleção de objetos simples.

Sabemos que $A_{0}$-mod é uma categoria abeliana e $k_{\mathbf{p}} \in \mathfrak{V}_{\mathbf{p}}$ é o único objeto simples de $\mathfrak{V}_{\mathrm{p}}$. Além disso, qualquer objeto em $\mathfrak{V}_{\mathrm{p}}$ se decompõe como soma direta do $A_{0}$-módulo $k_{\mathrm{p}}$. Portanto $\mathfrak{V}_{\mathbf{p}}$ é uma categoria semissimples.

Vamos definir mais alguns conceitos que serão úteis posteriormente.

Definição 2.5.2. Sejam $R$ e $S$ anéis, $f: S \rightarrow R$ um homomorfismo de anéis e $N$ um $R$-módulo à esquerda. Então $N$ tem estrutura de $S$-módulo à esquerda definida como segue: se $a \in S$ e $n \in N$ então an $:=f(a) n$. Dizemos que o $S$-módulo à esquerda ${ }_{S} N$ é obtido do $R$-módulo à esquerda ${ }_{R} N$ por restrição de escalares.

Em particular, se $f: S \rightarrow R$ é um homomorfismo de anéis então $R$ tem estrutura de $S$-módulo à esquerda como segue: se $a \in R$ e $b \in S$ então $b a:=f(b) a$ (Analogamente, $R$ tem estrutura de $S$-módulo à direita). Além disso, $R$ tem estrutura de $(R, S)$-bimódulo (denotada por ${ }_{R} R_{S}$ ) bem definida, pois a ação de $R$ em $R$ pela esquerda e a ação de $S$ em $R$ pela direita são relacionadas pela lei associativa, isto é,

$$
r \cdot\left(r^{\prime} \cdot s\right)=r \cdot\left(r^{\prime} \cdot f(s)\right)=\left(r \cdot r^{\prime}\right) \cdot f(s)=\left(r \cdot r^{\prime}\right) \cdot s
$$

para todos $r, r^{\prime} \in R, s \in S$. (Analogamente, $R$ tem estrutura de $(S, R)$-bimódulo). 
A restrição de escalares pode ser vista como um funtor $\operatorname{res}_{f}$, de $R$-módulos em $S$-módulos, chamado de funtor restrição. Um $R$-homomorfismo $u: M \rightarrow N$ automaticamente torna-se um $S$-homomorfismo entre as restrições de $M$ e $N$. De fato, se $m \in M$ e $a \in S$, então $u(a \cdot m)=u(f(a) \cdot m)=f(a) \cdot u(m)=a \cdot u(m)$.

Definição 2.5.3. Sejam $R$ e $S$ anéis, $f: S \rightarrow R$ um homomorfismo de anéis e $M$ um $S$ módulo à esquerda. Sabemos que $R$ tem estrutura de $(R, S)$-bimódulo, ou seja, ${ }_{R} R_{S}$. Existe o produto tensorial $R \otimes_{S} M$ que tem estrutura de $R$-módulo à esquerda dada por $b\left(b^{\prime} \otimes m\right)=$ $\left(b b^{\prime}\right) \otimes m$, para todos $b, b^{\prime} \in R, m \in M$. Dizemos que o $R$-módulo à esquerda $R \otimes_{S} M$ é obtido do $S$-módulo à esquerda $M$ por extensão de escalares. Chamamos o $R$-módulo à esquerda $R \otimes_{S} M$ de módulo induzido.

Intuitivamente, estender os escalares significa transformar um módulo sobre um anel $S$ em um módulo sobre um anel $R$, através de um homomorfismo de anéis de $S$ para $R$. O novo módulo admite mais escalares do que o original, por isso o nome "extensão".

A extensão de escalares pode ser interpretada como um funtor $i n d_{f}$, de $S$-módulos em $R$-módulos, chamado de funtor indução. Ele envia $M$ em $R \otimes_{S} M$ e um $S$-homomorfismo $u: M \rightarrow N$ em um $R$-homomorfismo $u_{R}: R \otimes_{S} M \rightarrow R \otimes_{S} N$, definido por $u_{R}=i d_{R} \otimes u$.

Observação 2.5.2. Se $R$ é um anel comutativo então um $R$-módulo é automaticamente um R-bimódulo. Em particular, se $M$ e $N$ são $R$-módulos, em que $R$ é um anel comutativo, então $M \otimes_{R} N$ é um $R$-módulo. Nesse caso, $M \otimes_{R} N \cong N \otimes_{R} M$.

Observação 2.5.3. Se $R, S$ são anéis e $M$ é um $(R, S)$-bimódulo. Então $R \otimes_{R} M \cong M \cong$ $M \otimes_{S} S$.

Definição 2.5.4. Sejam $R$ e $S$ anéis, $f: S \rightarrow R$ um homomorfismo de anéis e $M$ um $S$-módulo à esquerda. Sabemos que $R$ tem estrutura de $(S, R)$-bimódulo, ou seja, ${ }_{S} R_{R}$. Então $\operatorname{Hom}_{S}(R, M)$ é um $R$-módulo à esquerda por $(a \phi)\left(a^{\prime}\right)=\phi\left(a^{\prime} a\right)$, para a, $a^{\prime} \in R$ e $\phi \in$ $\operatorname{Hom}_{S}(R, M)$. Chamamos o $R$-módulo à esquerda $\operatorname{Hom}_{S}(R, M)$ de módulo coinduzido.

Também podemos definir um funtor coind $_{f}$, de $S$-módulos em $R$-módulos, que envia $M$ em $\operatorname{Hom}_{S}(R, M)$ e é chamado de funtor coindução. Para um $S$-homomorfismo $u: M \rightarrow N$ temos o $R$-homomorfismo $\operatorname{Hom}_{S}(R, u): \operatorname{Hom}_{S}(R, M) \rightarrow \operatorname{Hom}_{S}(R, N)$.

Vejamos as relações entres os funtores $r e s_{f}$, ind $d_{f}$ e coind $_{f}$. Para mais detalhes indicamos [Bro82].

Primeiro veremos que o funtor indução $i n d_{f}$ é adjunto à esquerda para o funtor restrição res . $_{\text {. }}$

Seja $R \otimes_{S} M$ o $R$-módulo à esquerda obtido de $M$ por extensão de escalares de $S$ para $R$, através do homomorfismo $f: S \rightarrow R$. Ele é um "enlargamento" de $M$ no sentido que existe uma aplicação canônica de $S$-módulos $i: M \rightarrow R \otimes_{S} M$ dado por $m \mapsto 1 \otimes m$. A aplicação $i$ é universal para $S$-aplicações de $M$ em um $R$-módulo no seguinte sentido: dado um $R$-módulo à esquerda $N$ (observamos que $N$ é um $S$-módulo à esquerda por restrição de escalares) e uma aplicação de $S$-módulos $u: M \rightarrow N$, existe uma única aplicação de $R$-módulos $g: R \otimes_{S} M \rightarrow N$ tal que $g i=u$. O diagrama abaixo ilustra essa situação:

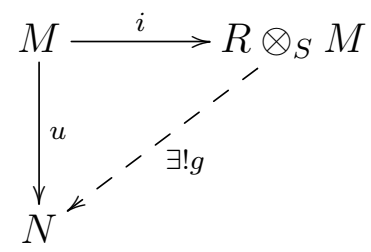


Isto nos diz que o funtor indução (extensão de escalares) é adjunto à esquerda para o funtor restrição de escalares. Podemos ver essa conexão de uma maneira mais explícita.

Novamente consideremos $f: S \rightarrow R$ um homomorfismo de anéis, $M$ um $S$-módulo à esquerda e $N$ um $R$-módulo à esquerda. Seja $u \in \operatorname{Hom}_{S}(M, N)$, em que $N$ é um $S$-módulo à esquerda via restrição de escalares. Definimos $F_{u}: R \otimes_{S} M \rightarrow N$ como sendo a composição

$$
R \otimes_{S} M \stackrel{i d_{R} \otimes u}{\longrightarrow} R \otimes_{S} N \longrightarrow N
$$

em que a última aplicação é definida por $r \otimes n \mapsto r n$. Temos que $F_{u}$ é um $R$-homomorfismo e então $F: \operatorname{Hom}_{S}(M, N) \rightarrow \operatorname{Hom}_{R}\left(R \otimes_{S} M, N\right)$ está bem definido e é um homomorfismo de grupos abelianos. Existe um homomorfismo inverso $G: \operatorname{Hom}_{R}\left(R \otimes_{S} M, N\right) \rightarrow \operatorname{Hom}_{S}(M, N)$ definido como segue. Seja $v \in \operatorname{Hom}_{R}\left(R \otimes_{S} M, N\right)$. Então $G_{v}$ é a composição

$$
M \longrightarrow S \otimes_{S} M \stackrel{f \otimes i d_{M}}{\longrightarrow} R \otimes_{S} M \stackrel{v}{\longrightarrow} N
$$

em que a primeira aplicação é o isomorfismo canônico $m \mapsto 1 \otimes m$.

Esta construção mostra que os grupos $\operatorname{Hom}_{R}\left(R \otimes_{S} M, N\right)$ e $\operatorname{Hom}_{S}(M, N)$ são isomorfos. Na verdade, este isomorfismo depende somente do homomorfismo $f$, e então ele é funtorial. Na linguagem da teoria de categorias, o funtor indução $i n d_{f}$ é adjunto à esquerda para o funtor restrição de escalares $\operatorname{res}_{f}$, ou seja,

$$
\operatorname{Hom}_{R}\left(\operatorname{ind}_{f}(M), N\right) \cong \operatorname{Hom}_{S}\left(M, \operatorname{res}_{f}(N)\right)
$$

para quaisquer $S$-módulo à esquerda $M$ e $R$-módulo à esquerda $N$.

Temos também que o funtor coindução coind $_{f}$ é adjunto à direita para o funtor restrição res .

Seja $\operatorname{Hom}_{S}(R, M)$ o $R$-módulo à esquerda obtido de $M$ por coextensão de escalares de $S$ para $R$, através do homomorfismo $f: S \rightarrow R$. Ele é um "enlargamento" de $M$ no sentido que existe uma aplicação canônica de $S$-módulos $p: \operatorname{Hom}_{S}(R, M) \rightarrow M$, dada por $f \mapsto f(1)$. A aplicação $p$ é universal para $S$-aplicações de um $R$-módulo para $M$ no seguinte sentido: dado um $R$-módulo $N$ e uma aplicação de $S$-módulos $u: N \rightarrow M$, existe uma única aplicação de $R$-módulos $g: N \rightarrow \operatorname{Hom}_{S}(R, M)$ tal que $p g=u$. O diagrama abaixo descreve a situação:

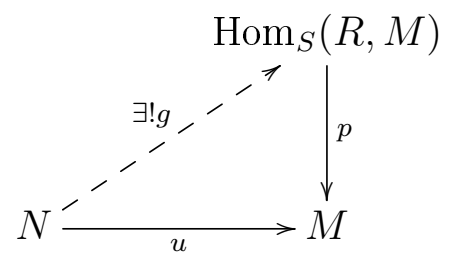

Mais concisamente, $\operatorname{Hom}_{R}\left(N, \operatorname{Hom}_{S}(R, M)\right) \cong \operatorname{Hom}_{S}(N, M)$, ou seja,

$$
\operatorname{Hom}_{R}\left(N, \operatorname{coind}_{f}(M)\right) \cong \operatorname{Hom}_{S}\left(\operatorname{res}_{f}(N), M\right)
$$

para quaisquer $S$-módulo $M$ e $R$-módulo $N$. Isto nos diz que o funtor coindução é adjunto à direita para o funtor restrição. Concluímos assim que o funtor restrição tem tanto um adjunto à esquerda quanto um adjunto à direita.

Baseado nas definições dadas acima vamos definir dois funtores: um de $\mathfrak{W}$ em $\mathfrak{V}_{\mathbf{p}}$ e $o$ outro de $\mathfrak{V}_{\mathbf{p}}$ em $\mathfrak{W}$, em que $\mathfrak{W}$ é uma subcategoria plena da categoria $A$-mod e $\mathfrak{V}_{\mathbf{p}}$ é uma subcategoria plena da categoria $A_{0}$-mod, definidas anteriormente.

Como $A_{0}$ é $k$-subálgebra de $A$ temos o homomorfismo inclusão $\iota: A_{0} \rightarrow A$. Então um 
$A$-módulo $M$, tem estrutura de $A_{0}$-módulo, por $a m=\iota(a) m$, para todos $a \in A_{0}, m \in M$.

Seja $M$ um $A$-módulo de peso, ou seja, $M=\bigoplus_{\mathbf{p} \in k^{\mathrm{I}}} M_{\mathbf{p}}$.

$M \in A_{0}$-mod, restringindo os escalares. Além disso, cada espaço de peso $M_{\mathbf{p}}$ é um $A_{0^{-}}$ módulo, visto que $A_{0}$ é uma $k$-álgebra comutativa. Temos também que $\mathfrak{m}_{\mathbf{p}} \cdot M_{\mathbf{p}}=\{0\}$. Portanto podemos definir o funtor restrição

$$
R e s_{\mathbf{p}}: \mathfrak{W} \rightarrow \mathfrak{V}_{\mathbf{p}}
$$

tal que $M \mapsto \operatorname{Res}_{\mathbf{p}}(M)=M_{\mathbf{p}}$ e para um homomorfismo $f: M \rightarrow M^{\prime}$ em $\mathfrak{W}$, ou seja, $f: \bigoplus_{\mathbf{p} \in k^{\mathrm{I}}} M_{\mathbf{p}} \rightarrow \bigoplus_{\mathbf{p} \in k^{\mathrm{I}}} M_{\mathbf{p}}^{\prime}$, temos que $\operatorname{Res}_{\mathbf{p}}(f)=f_{\mathbf{p}}: M_{\mathbf{p}} \rightarrow M_{\mathbf{p}}^{\prime}$ é um homomorfismo em $\mathfrak{V}_{\mathbf{p}}$. Observamos que se $\mathbf{p} \notin \operatorname{supp}(M)$, então $\operatorname{Res}_{\mathbf{p}}(M)=\{0\}$.

Seja $0 \longrightarrow M^{\prime} \stackrel{f}{\rightarrow} M \stackrel{g}{\rightarrow} M^{\prime \prime} \rightarrow 0$ uma sequência exata de $A$-módulos de peso. Observamos que para um homomorfismo de $A$-módulos de peso $f: M \rightarrow M^{\prime}$ temos que $\operatorname{Res}_{\mathbf{p}}(k e r(f))$ $=\operatorname{ker}\left(\operatorname{Res}_{\mathbf{p}}(f)\right)=\operatorname{ker}\left(f_{\mathbf{p}}\right)$ e $\operatorname{Res}_{\mathbf{p}}(i m(f))=i m\left(\operatorname{Res}_{\mathbf{p}}(f)\right)=i m\left(f_{\mathbf{p}}\right)$. Aplicando o funtor $R e s_{\mathbf{p}}$ na sequência exata obtemos a sequência $0 \longrightarrow M_{\mathbf{p}}^{\prime} \stackrel{f_{\mathbf{p}}}{\longrightarrow} M_{\mathbf{p}} \stackrel{g_{\mathbf{p}}}{\longrightarrow} M_{\mathbf{p}}^{\prime \prime} \longrightarrow 0$. Como $\operatorname{ker}(f)=\{0\}$ temos que $\operatorname{Res}_{\mathbf{p}}(\operatorname{ker}(f))=\operatorname{Res}_{\mathbf{p}}(\{0\})=\{0\}$. Assim $\operatorname{ker}\left(f_{\mathbf{p}}\right)=\{0\}$. Como $i m(g)=M^{\prime \prime}$ temos que $\operatorname{Res}_{\mathbf{p}}(i m(g))=M_{\mathbf{p}}^{\prime \prime}$. Assim $i m\left(g_{\mathbf{p}}\right)=M_{\mathbf{p}}^{\prime \prime}$. Como $i m(f)=k e r(g)$ te$\operatorname{mos}$ que $i m\left(f_{\mathbf{p}}\right)=\operatorname{Res}_{\mathbf{p}}(i m(f))=\operatorname{Res}_{\mathbf{p}}(\operatorname{ker}(g))=\operatorname{ker}\left(g_{\mathbf{p}}\right)$. Então ao aplicar o funtor $\operatorname{Res}_{\mathbf{p}}$ em uma sequência exata, obtemos uma sequência exata. Portanto o funtor $R_{e} s_{\mathbf{p}}$ é exato.

Agora vamos definir um outro funtor.

Como $A_{0}$ é $k$-subálgebra de $A$, podemos afirmar que $A$ tem estrutura de $\left(A, A_{0}\right)$-bimódulo, ou seja, ${ }_{A} A_{A_{0}}$. Seja $N \in \mathfrak{V}_{\mathrm{p}}$. Temos que $A \otimes_{A_{0}} N$ existe e tem estrutura de $A$-módulo à esquerda por $a\left(a^{\prime} \otimes n\right)=\left(a a^{\prime}\right) \otimes n$, para todos $a, a^{\prime} \in A, n \in N$. Portanto $A \otimes_{A_{0}} N \in A$-mod.

Observamos que $A \otimes_{A_{0}} N$ é gerado pelo conjunto $1 \otimes N=\{1 \otimes n \mid n \in N\}$, como $A$-módulo à esquerda. Para todo $1 \otimes n \in 1 \otimes N$, temos que $\mathfrak{m}_{\mathbf{p}} \cdot(1 \otimes n)=1 \otimes \mathfrak{m}_{\mathbf{p}} \cdot n=0$. Então $1 \otimes N \subseteq\left(A \otimes_{A_{0}} N\right)_{\mathbf{p}}$.

Para $i \in \mathrm{I}$, aplicando os geradores $X_{i}, Y_{i}$ de $A$ em um elemento $1 \otimes n$ temos que $X_{i} \cdot(1 \otimes n)$ $\in\left(A \otimes_{A_{0}} N\right)_{\mathbf{p}+\mathbf{e}_{i}}$ e $Y_{i} \cdot(1 \otimes n) \in\left(A \otimes_{A_{0}} N\right)_{\mathbf{p}-\mathbf{e}_{i}}$, como visto na seção anterior. Pela Proposição 1.3.2 (Capítulo 1 ), cada $a \in A$ é da forma

$$
a=\sum_{\mathbf{v} \in \mathbb{Z}_{f}^{\mathrm{I}}} \mathbf{X}_{\mathbf{v}} a_{\mathbf{v}}
$$

em que cada $a_{\mathbf{v}} \in A_{0}$. Então

$$
a(1 \otimes n)=a \otimes n=\sum_{\mathbf{v} \in \mathbb{Z}_{f}^{\mathrm{I}}} \mathbf{X}_{\mathbf{v}} a_{\mathbf{v}} \otimes n=\sum_{\mathbf{v} \in \mathbb{Z}_{f}^{\mathrm{I}}} \mathbf{X}_{\mathbf{v}} \otimes a_{\mathbf{v}} \cdot n=\sum_{\mathbf{v} \in \mathbb{Z}_{f}^{\mathrm{I}}} \mathbf{X}_{\mathbf{v}} \otimes n_{\mathbf{v}}
$$

em que cada $n_{\mathbf{v}} \in N$. Pelas observações da seção anterior, $a \otimes n \in \sum_{\mathbf{v}^{\prime} \in \mathbb{Z}_{f}^{\mathrm{I}}}\left(A \otimes_{A_{0}} N\right)_{\mathbf{p}+\mathbf{v}^{\prime}}$.

Podemos considerar que $A \otimes_{A_{0}} N=\operatorname{span}\{a \otimes n \mid a \in A, n \in N\}$ como $k$-espaço vetorial (ver Apêndice B, Obs. B.3.2). Portanto $A \otimes_{A_{0}} N$ é um $A$-módulo de peso, isto é, $A \otimes_{A_{0}} N$ $\in \mathfrak{W}$. Além disso, $\operatorname{supp}\left(A \otimes_{A_{0}} N\right) \subseteq \mathbf{p}+\mathbb{Z}_{f}^{\mathrm{I}}$, ou seja, $A \otimes_{A_{0}} N \in \mathfrak{W}_{\mathbf{p}}$. Portanto podemos definir o funtor indução

$$
\operatorname{Ind}_{\mathbf{p}}=: A \otimes_{A_{0}}-: \mathfrak{V}_{\mathbf{p}} \rightarrow \mathfrak{W}
$$

tal que $N \mapsto \operatorname{Ind}_{\mathbf{p}}(N)=A \otimes_{A_{0}} N$ e para um homomorfismo $f: N \rightarrow N^{\prime}$ em $\mathfrak{V}_{\mathbf{p}}$ temos que $\operatorname{Ind}_{\mathbf{p}}(f)=A \otimes_{A_{0}} f=i d_{A} \otimes f: A \otimes_{A_{0}} N \rightarrow A \otimes_{A_{0}} N^{\prime}$ é um homomorfismo em $\mathfrak{W}$.

Vamos mostrar que o funtor $I_{n d}$ é exato. Já sabemos que o funtor $A \otimes_{A_{0}}$ - é exato à direita (ver Apêndice $\mathrm{B}$, Teorema B.3.19). Precisamos mostrar que é exato à esquerda, 
isto é, que preserva a injetividade. Em outras palavras: se $M \hookrightarrow M^{\prime}$ é injetor implica que $A \otimes_{A_{0}} M \hookrightarrow A \otimes_{A_{0}} M^{\prime}$ é injetor.

Seja $0 \longrightarrow N^{\prime} \stackrel{f}{\longrightarrow} N \stackrel{g}{\rightarrow} N^{\prime \prime} \longrightarrow 0$ uma sequência exata em $\mathfrak{V}_{\mathbf{p}}$. Aplicando o funtor $\operatorname{Ind}_{\mathbf{p}}$ nessa sequência obtemos $0 \longrightarrow A \otimes_{A_{0}} N^{\prime} \stackrel{i d_{A} \otimes f}{\longrightarrow} A \otimes_{A_{0}} N \stackrel{i d_{A} \otimes g}{\longrightarrow} A \otimes_{A_{0}} N^{\prime \prime} \longrightarrow 0$. Vamos mostrar que $i d_{A} \otimes f$ é um monomorfismo.

Vimos que $A$ é livre como $A_{0}$-módulo (Capítulo 1, Proposição 1.3.2). Então $A \cong \bigoplus_{j \in J} A_{0}$ como $A_{0}$-módulos. Consideremos então a aplicação $\left(\bigoplus_{j \in J} A_{0}\right) \otimes_{A_{0}} N^{\prime} \stackrel{i d_{A} \otimes f}{\longrightarrow}\left(\bigoplus_{j \in J} A_{0}\right) \otimes_{A_{0}} N$. Usando o Teorema B.3.20 (Apêndice B) temos que:

$$
\left(\bigoplus_{j \in J} A_{0}\right) \otimes_{A_{0}} N^{\prime} \cong \bigoplus_{j \in J}\left(A_{0} \otimes_{A_{0}} N^{\prime}\right) \cong \bigoplus_{j \in J} N^{\prime}
$$

Assim, o seguinte diagrama comuta:

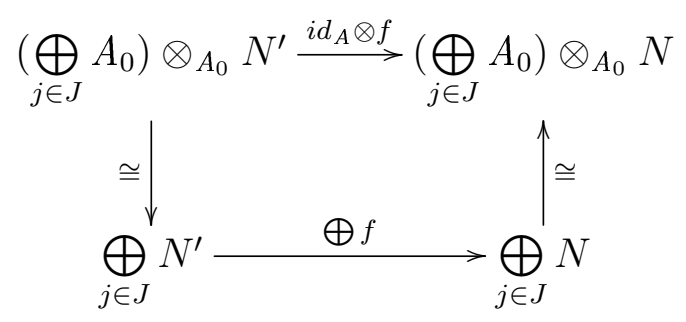

Como $f$ é um monomorfismo, temos que $\bigoplus f$ é um monomorfismo. Então $i d_{A} \otimes f$ é um monomorfismo. Portanto o funtor $\operatorname{Ind}_{\mathbf{p}}$ é exato.

Como mostrado anteriormente o funtor $\operatorname{Ind}_{\mathbf{p}}$ é adjunto à esquerda para o funtor $R e s_{\mathbf{p}} \mathrm{e}$ o funtor $R e s_{\mathbf{p}}$ é adjunto à direita para o funtor $I n d_{\mathbf{p}}$, ou seja, $I n d_{\mathbf{p}}$ e $R e s_{\mathbf{p}}$ são um par de funtores adjuntos exatos. Portanto

$$
\operatorname{Hom}_{\mathfrak{W}}\left(\operatorname{Ind}_{\mathbf{p}}(N), M\right) \cong \operatorname{Hom}_{\mathfrak{V}_{\mathbf{p}}}\left(N, \operatorname{Res}_{\mathbf{p}}(M)\right)
$$

para quaisquer $N \in \mathfrak{V}_{\mathrm{p}}, M \in \mathfrak{W}$.

Além disso, o funtor $I n d_{\mathbf{p}}$ aplica objetos projetivos de $\mathfrak{V}_{\mathbf{p}}$ em objetos projetivos de $\mathfrak{W}$. Seja $N$ um $A_{0}$-módulo projetivo em $\mathfrak{V}_{\mathbf{p}}$. Então $\operatorname{Hom}_{\mathfrak{V}_{\mathbf{p}}}(N, \cdot)$ é exato em $\mathfrak{V}_{\mathbf{p}}$. Logo $\operatorname{Hom}_{\mathfrak{W}}\left(A \otimes_{A_{0}} N, \cdot\right)$ é exato em $\mathfrak{W}$. Portanto $A \otimes_{A_{0}} N=\operatorname{Ind} d_{\mathbf{p}}(N)$ é um $A$-módulo projetivo em $\mathfrak{W}$.

Pelo Corolário B.3.6 (Apêndice B) temos que:

$$
A \otimes_{A_{0}} A_{0} / \mathfrak{m}_{\mathbf{p}} \cong A / A \mathfrak{m}_{\mathbf{p}}
$$

Então:

$$
P(\mathbf{p}):=\operatorname{Ind}_{\mathbf{p}}\left(k_{\mathbf{p}}\right)=A \otimes_{A_{0}} A_{0} / \mathfrak{m}_{\mathbf{p}} \cong A / A \mathfrak{m}_{\mathbf{p}}
$$

é projetivo em $\mathfrak{W}$, ou seja, $P(\mathbf{p})$ é um $A$-módulo de peso projetivo.

Além disso, por adjunção, para qualquer $M \in \mathfrak{W}$ temos um isomorfismo natural $\operatorname{Hom}_{\mathfrak{W}}(P(\mathbf{p}), M) \cong M_{\mathbf{p}}$. Vejamos as justificativas.

Seja $R$ um anel. Suponha que ${ }_{R} M$ e ${ }_{R} N$ são $R$-módulos e que $I$ é um ideal de $R$ que aniquila tanto $M$ quanto $N$. Então $M$ e $N$ carregam uma estrutura natural de $R / I$-módulo (isto será discutido posteriormemte) e certamente todo $R$-homomorfismo entre eles é um $R / I$-homomorfismo. Então temos que $\operatorname{Hom}_{R}(M, N)=\operatorname{Hom}_{R / I}(M, N)$. Agora vamos mostrar que $\operatorname{Hom}_{R}(R, M) \cong M$.

Seja $\phi: \operatorname{Hom}_{R}(R, M) \rightarrow M$ definida por $\phi(\varphi)=\varphi\left(1_{R}\right) . \operatorname{Sejam} \varphi, \psi \in \operatorname{Hom}_{R}(R, M)$ e $r$ 
$\in R$. Então $\phi(\varphi+r \cdot \psi)=(\varphi+r \cdot \psi)\left(1_{R}\right)=\phi(\varphi)+r \cdot \phi(\psi)$. Assim $\phi$ é um $R$-homomorfismo. Suponhamos agora que $\varphi \in \operatorname{ker}(\phi)$. Então para todo $r \in R, \varphi(r)=r \cdot \varphi\left(1_{R}\right)=r \cdot \phi(\varphi)=$ $r \cdot 0=0$. Assim $\varphi=0$, e então $\phi$ é injetora. Por fim, seja $m \in M$. Defina $\psi_{m}: R \rightarrow M$, por $\psi_{m}(r)=r \cdot m$. Como para todos $x, y, r \in R$ temos que $\psi_{m}(x+r \cdot y)=(x+r \cdot y) \cdot m=$ $\psi_{m}(x)+r \cdot \psi_{m}(y)$, temos que $\psi_{m}$ é um $R$-homomorfismo. $\operatorname{Assim} \psi_{m} \in \operatorname{Hom}_{R}(R, M)$. Além disso, $\phi\left(\psi_{m}\right)=\psi_{m}\left(1_{R}\right)=1_{R} \cdot m=m$. Então $\phi$ é sobrejetora. Portanto $\operatorname{Hom}_{R}(R, M) \cong M$.

Usando o par de funtores adjuntos $I n d_{\mathbf{p}}$ e $R e s_{\mathbf{p}}$, temos que:

$$
\operatorname{Hom}_{\mathfrak{W}}(P(\mathbf{p}), M) \cong \operatorname{Hom}_{\mathfrak{V}_{\mathbf{p}}}\left(A_{0} / \mathfrak{m}_{\mathbf{p}}, \operatorname{Res}_{\mathbf{p}}(M)\right)=\operatorname{Hom}_{\mathfrak{V}_{\mathbf{p}}}\left(A_{0} / \mathfrak{m}_{\mathbf{p}}, M_{\mathbf{p}}\right)
$$

Como $A_{0} / \mathfrak{m}_{\mathbf{p}}$ e $M_{\mathbf{p}}$ são $A_{0} / \mathfrak{m}_{\mathbf{p}}$-módulos à esquerda, pelas justificativas dadas acima, $\operatorname{Hom}_{\mathfrak{V}_{\mathbf{p}}}\left(A_{0} / \mathfrak{m}_{\mathbf{p}}, M_{\mathbf{p}}\right) \cong M_{\mathbf{p}}$. Portanto, para todo $M \in \mathfrak{W}$ temos:

$$
\operatorname{Hom}_{\mathfrak{W}}(P(\mathbf{p}), M) \cong M_{\mathbf{p}}
$$

Queremos definir o topo do $A$-módulo $P(\mathbf{p})$. Antes de definir o topo top $(M)$ de um $R$ módulo $M$, precisamos definir o radical do $R$-módulo $M$. Vamos relacionar alguns resultados importantes e as demonstrações podem ser encontradas em [Lam01] e [Bea99].

Em 1943, Jacobson desenvolveu uma teoria de estrutura de anéis sem hipóteses de finitude. Considerando-se um módulo simples sobre o anel $R$ como uma representação do anel, ele definiu o que hoje é conhecido como o radical de Jacobson $(\operatorname{rad}(R))$, isto é, a intersecção dos kernels de todas as representações de $R$ para $R$-módulos à esquerda simples. Um anel $R$ é chamado de semissimples de Jacobson se $\operatorname{rad}(R)=\{0\}$. O $\operatorname{rad}(R)$ é um ideal bilateral de $R$ e $\operatorname{rad}(R / \operatorname{rad}(R))=\{\overline{0}\}$, ou seja, $R / \operatorname{rad}(R)$ é um anel semissimples de Jacobson.

Um ponto de vista um pouco mais geral, é considerar radicais para módulos. Em particular, o principal interesse é saber como um determinado módulo está relacionado com módulos simples. Um submódulo é minimal se, e somente se, ele é simples; é maximal se, e somente se, o módulo quociente correspondente é simples.

Temos que a soma de todos os submódulos minimais de $M$ é o socle de $M$, denotado por $\operatorname{soc}(M)$. O módulo $M$ é chamado de semissimples se ele pode ser expresso como uma soma de submódulos minimais. Também dizemos que $M$ é um $R$-módulo à esquerda semissimples (ou completamente redutível) se todo $R$-submódulo de $M$ é um somando direto de $M$.

Teorema 2.5.1. Sejam $R$ um anel e $M$ um $R$-módulo à esquerda. Então as seguintes propriedades são equivalentes:

1. M é semissimples.

2. $M$ é a soma direta de uma família de submódulos simples.

3. $M$ é a soma de uma família de submódulos simples.

Demonstração. [Lam01], Capítulo 1, Teorema 2.4, pg 27.

Vejamos a definição do radical de Jacobson de um módulo:

Definição 2.5.5. Sejam $R$ um anel e $M$ um $R$-módulo à esquerda. $O$ radical de Jacobson de $M$, denotado por rad $(M)$, é a intersecção de todos os $R$-submódulos à esquerda maximais de $M$. Se $M$ não possui submódulos maximais, então $\operatorname{rad}(M)=M$. 
Observamos que módulos que não sejam finitamente gerados, podem não ter submódulos maximais. Nesse caso $\operatorname{rad}(M)=M$.

O socle e o radical de Jacobson de um módulo podem ser caracterizados de várias maneiras.

O radical de Jacobson é um caso particular da teoria dos radicais. É importante ressaltar que é possível estudar radicais de módulos a partir de um ponto de vista mais geral (para mais detalhes [Bea99], Capítulo 3). Existe também uma extensa (mas bastante diferente) teoria de radicais de anéis. A ideia geral é a de fatorar uma "parte ruim" em algum sentido. No caso do radical de Jacobson, espera-se que a parte que resta, $M / \operatorname{rad}(M)$, é o mais próximo possível de um módulo semissimples.

Algumas propriedades do radical estão reunidas nas seguintes proposições:

\section{Proposição 2.5.1.}

1. Sejam $R$ um anel e $M, M^{\prime} R$-módulos à esquerda. Então: $M^{\prime} \subseteq M \Rightarrow \operatorname{rad}\left(M^{\prime}\right) \subseteq$ $\operatorname{rad}(M)$.

2. Seja $\left\{M_{i}\right\}_{i \in I}$ uma família de $R$-módulos. Então: $\operatorname{rad}\left(\bigoplus_{i \in I} M_{i}\right)=\bigoplus_{i \in I} \operatorname{rad}\left(M_{i}\right)$.

3. Se F é um $R$-módulo à esquerda livre então $\operatorname{rad}(F)=\operatorname{rad}(R) F$.

Demonstração. [Lam01], Capítulo 8, Proposição 24.6, pg 359.

Proposição 2.5.2. Seja $R$ um anel. Suponha que $L, M$ e $N$ são $R$-módulos à esquerda finitamente gerados.

1. Um elemento $m \in M$ pertence à $\operatorname{rad}(M)$ se, e somente se, $f(m)=0$, para qualquer $f$ $\in \operatorname{Hom}_{R}(M, S)$ e qualquer $R$-módulo à esquerda simples $S$.

2. Se $f \in \operatorname{Hom}_{R}(M, N)$, ent $\tilde{a} o f(\operatorname{rad}(M)) \subseteq \operatorname{rad}(N)$.

3. $\operatorname{rad}(R) M=\operatorname{rad}(M)$.

4. Assuma que $L$ e $M$ são R-submódulos de $N$. Se $L \subseteq \operatorname{rad}(N)$ e $L+M=N$ então $M=N$.

Observamos que se $M$ é um $R$-módulo à esquerda e $I$ é um ideal de $R$, tal que $I \subseteq$ $\operatorname{ann}_{R}(M)$, podemos considerar $M$ como $(R / I)$-módulo à esquerda: se $\bar{a} \in R / I$ é representado por $a \in R$, defina $\bar{a} m$ como sendo $a m$, em que $m \in M$. Observe que esta definição independe da escolha do representante $a$ de $\bar{a}$ pois $I M=0$. De fato: seja $b \in R$ um outro representante tal que $\bar{a}=\bar{b}$. Então $\bar{a}-\bar{b}=\overline{0}$, ou seja, $a-b \in I$. Como $I M=0$, temos que $(a-b) m=0$, isto é, $a m=b m$. Portanto $\bar{a} m=\bar{b} m$.

O topo de um $R$-módulo à esquerda $M$ é o $R$-módulo à esquerda

$$
\operatorname{top}(M)=M / \operatorname{rad}(M)
$$

Como $\operatorname{rad}(R) M=\operatorname{rad}(M)$ então $\operatorname{rad}(R)(M / \operatorname{rad}(M))=\{\overline{0}\} ; \operatorname{assim}$ top $(M)$ também é $R / \operatorname{rad}(R)$-módulo à esquerda com respeito a ação de $R / \operatorname{rad}(R)$ definida pela fórmula $(a+\operatorname{rad}(R)) \cdot(m+\operatorname{rad}(M))=\operatorname{am}+\operatorname{rad}(M)$.

Observamos que se $f: M \rightarrow N$ é um $R$-homomorfismo então $f(\operatorname{rad}(M)) \subseteq \operatorname{rad}(N)$ e então $f$ induz um homomorfismo $\operatorname{top}(f): \operatorname{top}(M) \rightarrow \operatorname{top}(N)$ de $R / \operatorname{rad}(R)$-módulos definido pela fórmula $\operatorname{top}(f)(m+\operatorname{rad}(M))=f(m)+\operatorname{rad}(N)$. 
Antes de voltar nossa atenção para o $A$-módulo de peso projetivo $P(\mathbf{p})$, definido anteriormente, vamos analisar a relação entre um grupo formado por alguns automorfismos de $A_{0}$ e o grupo abeliano aditivo $\mathbb{Z}_{f}^{\mathrm{I}}$. Essa relação trará informações sobre a estrutura de $P(\mathbf{p})$.

Denotaremos por $\sigma_{i}$ o automorfismo de $A_{0}$ dado por:

$$
\sigma_{i}\left(t_{j}\right)=\left\{\begin{array}{lll}
t_{j} & \text { se } & i \neq j \\
t_{i}+1 & \text { se } & i=j
\end{array}\right.
$$

Seu inverso $\sigma_{i}^{-1}$ é definido da seguinte maneira:

$$
\sigma_{i}^{-1}\left(t_{j}\right)=\left\{\begin{array}{lll}
t_{j} & \text { se } & i \neq j \\
t_{i}-1 & \text { se } & i=j
\end{array}\right.
$$

Seja $H$ o grupo gerado por $\sigma_{i}$, para todo $i \in \mathrm{I}$. Sabemos que todo ideal maximal em $A_{0}$ tem a forma $\mathfrak{m}_{\mathbf{p}}$, para algum $\mathbf{p} \in k^{\mathrm{I}}$ (Lema 1.3.1, Capítulo 1). Dessa forma identificamos ideais maximais $\mathfrak{m}_{\mathbf{p}}$ em $A_{0}$ com elementos $\mathbf{p}$ em $k^{\mathrm{I}}$ ( $k$ é algebricamente fechado) da seguinte forma $\left(t_{i}-p_{i}\right) \leftrightarrow p_{i}$ para todo $i$, ou seja, existe uma relação biunívoca entre os ideais maximais de $A_{0}$ e os elementos de $k^{\mathrm{I}}$. Para cada $j \in \mathrm{I}, t_{j} \in A_{0}$ é identificado com $p_{j} \in k$, então $\sigma_{i}\left(p_{j}\right)=p_{j}$ se $i \neq j$, e $\sigma_{i}\left(p_{j}\right)=p_{i}+1$ se $i=j$. Assim para $\mathbf{p}=\left(p_{j}\right)_{j \in \mathrm{I}} \in k^{\mathrm{I}}$, temos que $\sigma_{i}(\mathbf{p})=\mathbf{p}+\mathbf{e}_{i}$, em que $\mathbf{e}_{i}$ tem 1 na $i$-ésima coordenada e 0 nas outras. De maneira análoga, $\sigma_{i}^{-1}(\mathbf{p})=\mathbf{p}-\mathbf{e}_{i}$. Podemos generalizar essa ideia. Seja $\sigma \in H$. Então $\sigma=\sigma_{i_{1}}^{r_{1}} \cdots \sigma_{i_{n}}^{r_{n}}$, em que $i_{1}, \ldots, i_{n} \in \mathrm{I}$ e somente uma quantidade finita $\operatorname{dos} r_{j}$ é não nula. Logo, $\sigma(\mathbf{p})=\mathbf{p}+\mathbf{v}$, para $\operatorname{algum} \mathbf{v} \in \mathbb{Z}_{f}^{\mathrm{I}}$.

Se $M$ é um $A$-módulo de peso e $x \in M$ tal que $x \in M_{\mathbf{p}}$, com $\mathbf{p} \in k^{\mathrm{I}}$, vimos na seção anterior que $X_{i} x \in M_{\mathbf{p}+\mathbf{e}_{i}}$ e $Y_{i} x \in M_{\mathbf{p}-\mathbf{e}_{i}}$, com $i \in \mathrm{I}$. Logo, para qualquer $\mathbf{p} \in k^{\mathrm{I}}$ temos que $X_{i} M_{\mathbf{p}} \subseteq M_{\sigma_{i}(\mathbf{p})}$ e $Y_{i} M_{\mathbf{p}} \subseteq M_{\sigma_{i}^{-1}(\mathbf{p})}$.

Observando as relações $\sigma_{i}(\mathbf{p})=\mathbf{p}+\mathbf{e}_{i}$ e $\sigma_{i}^{-1}(\mathbf{p})=\mathbf{p}-\mathbf{e}_{i}$, percebemos que a identificação entre os ideais maximais de $A_{0}$ e os elementos de $k^{\mathrm{I}}$, dá origem à uma identificação natural entre $\sigma_{i} \in H$ e $\mathbf{e}_{i} \in \mathbb{Z}_{f}^{\mathrm{I}}$. O mesmo vale para $\sigma_{i}^{-1}$ e $-\mathbf{e}_{i}$. Portanto $H \cong \mathbb{Z}_{f}^{\mathrm{I}}$.

$\mathrm{O}$ grupo $\mathbb{Z}_{f}^{\mathrm{I}}$ age livremente em $k^{\mathrm{I}}$. Além disso, recordemos que os elementos de $\mathbb{Z}_{f}^{\mathrm{I}}$ são naturalmente índices da base livre $\left\{\mathbf{X}_{\mathbf{v}} \mid \mathbf{v} \in \mathbb{Z}_{f}^{\mathrm{I}}\right\}$ de $A$ com um $A_{0}$-módulo.

$P(\mathbf{p})$ é $A$-módulo de peso, com $\operatorname{supp}(P(\mathbf{p})) \subseteq \mathbf{p}+\mathbb{Z}_{f}^{\mathrm{I}}$. Logo:

$$
P(\mathbf{p})=\bigoplus_{\mathbf{v} \in \mathbb{Z}_{f}^{\mathrm{I}}} P(\mathbf{p})_{\mathbf{p}+\mathbf{v}}
$$

De acordo com a discussão acima, temos que:

$$
P(\mathbf{p}) \cong \bigoplus_{\sigma \in \mathbb{Z}_{f}^{\mathrm{I}}} P(\mathbf{p})_{\sigma(\mathbf{p})}
$$

Como $\mathbb{Z}_{f}^{\mathrm{I}} \cong H$, temos que todos os $\sigma(\mathbf{p})$ são diferentes entre si.

Observamos que $P(\mathbf{p})_{\mathbf{p}}=\operatorname{Res}_{\mathbf{p}}(P(\mathbf{p}))=A_{0} \otimes_{A_{0}} k_{\mathbf{p}} \cong k_{\mathbf{p}}$. Sabemos que $k_{\mathbf{p}}:=A_{0} / \mathfrak{m}_{\mathbf{p}}$, então para $i \in \mathrm{I}, t_{i}$ é congruente a $p_{i}\left(\bmod \mathfrak{m}_{\mathbf{p}}\right)$, em que $p_{i} \in k$. Podemos construir uma identificação entre $k_{\mathrm{p}}$ e $k$, ou seja, eles são isomorfos como $k$-espaços vetoriais. Portanto $P(\mathbf{p})_{\mathbf{p}}$ é unidimensional sobre $k$. Logo, para qualquer $\sigma \in \mathbb{Z}_{f}^{\mathrm{I}}, P(\mathbf{p})_{\sigma(\mathbf{p})}$ é unidimensional sobre $k$.

Agora que sabemos algo sobre a estrutura dos $A$-módulos de peso projetivos $P(\mathbf{p})$, vamos seguir definindo alguns conceitos importantes, para no fim desta seção mostrar que $P(\mathbf{p})$ é 
um $A$-módulo indecomponível e ele é a cobertura projetiva do seu topo.

Definição 2.5.6. Seja $R$ um anel. Um $R$-módulo à esquerda $M \neq\{0\}$ é indecomponível se ele não possui submódulos não triviais $M_{1}$ e $M_{2}$ tais que $M=M_{1} \bigoplus M_{2}$.

Definição 2.5.7. Um anel $R$ (não necessariamente comutativo) é local se ele tem um único ideal à esquerda maximal.

Equivalentemente, podemos dizer que um anel $R$ é um anel local se o conjunto formado pelos elementos não inversíveis de $R$ (isto é, $R \backslash \mathcal{U}(R)$, tal que $\mathcal{U}(R)$ é o grupo das unidades de $R$ ) for um ideal.

Os corpos são anéis locais com um único ideal à esquerda maximal $\{0\}$ (nesse caso é um ideal bilateral, pois o anel é comutativo).

Definição 2.5.8. Um idempotente em um anel $R$ é um elemento e tal que $e^{2}=e$. Se e, $f$ são idempotentes em um anel $R$, dizemos que e, $f$ são idempotentes ortogonais se ef $=f e=0$.

Proposição 2.5.3. Sejam $R$ um anel e $M$ um $R$-módulo.

1. Seja $M=M_{1} \bigoplus \cdots \bigoplus M_{r}$ uma decomposição de $M$ em soma direta de submódulos. Sejam $\pi_{1}, \ldots, \pi_{r}$ as projeções e $\iota_{1}, \ldots, \iota_{r}$ as inclusões canônicas associadas à essa decomposiçãa. Para cada $j=1, \ldots, r$, seja $e_{j}=\iota_{j} \pi_{j}$. Tem-se então que os elementos $e_{1}, \ldots, e_{r} \in \operatorname{End}_{R}(M)$ são idempotentes dois a dois ortogonais e tais que $e_{1}+\cdots+e_{r}=i d_{M}$.

2. Sejam $e_{1}, \ldots, e_{r} \in \operatorname{End}_{R}(M)$ idempotentes dois a dois ortogonais e tais que $e_{1}+\cdots+$ $e_{r}=i d_{M}$. Tem-se então que $M=M_{1} \oplus \cdots \bigoplus M_{r}$, em que $M_{i}=e_{i}(M)$, para todo $i=1, \ldots, r$.

Demonstração. 1. Para $m=m_{1}+\cdots+m_{r} \in M$, temos que $e_{j}(m)=\iota_{j} \pi_{j}(m)=m_{j}$. Então $m=e_{1}(m)+\cdots+e_{r}(m)=\left(e_{1}+\cdots+e_{r}\right)(m)$, isto é, $e_{1}+\cdots+e_{r}=i d_{M}$. Além disso, $e_{j}^{2}(m)=e_{j}\left(e_{j}(m)\right)=e_{j}\left(m_{j}\right)=m_{j}=e_{j}(m)$, ou seja, $e_{j}^{2}=e_{j}$ e para $j \neq k$, $e_{j} e_{k}(m)=e_{j}\left(m_{k}\right)=0$.

2. Claramente, para $i=1, \ldots, r, M_{i}=e_{i}(M) \subseteq M$. Para todo $m \in M, m=i d_{M}(m)=$ $\left(e_{1}+\cdots+e_{r}\right)(m)=e_{1}(m)+\cdots+e_{r}(m)$. Portanto $M=M_{1}+\cdots+M_{r}$. Para $m \in$ $M$, seja $m=m_{1}+\cdots+m_{r}, \operatorname{com} m_{i} \in M_{i}=e_{i}(M)$. Como $m_{i} \in e_{i}(M)$, existe $n_{i} \in$ $M$ tal que $e_{i}\left(n_{i}\right)=m_{i}$. Então $e_{i}(m)=e_{i}\left(m_{1}+\cdots+m_{r}\right)=e_{i}\left(e_{1}\left(n_{1}\right)+\cdots+e_{r}\left(n_{r}\right)\right)=$ $e_{i}\left(n_{i}\right)=m_{i}$. Portanto $m$ é escrito de maneira única, ou seja, a soma é direta. Assim $M=M_{1} \oplus \cdots \bigoplus M_{r}$.

Proposição 2.5.4. Um R-módulo $M$ é indecomponível se, e somente se, 0 e $i d_{M}$ forem os únicos idempotentes de $\operatorname{End}_{R}(M)$.

Demonstração. Seja $e \in \operatorname{End}_{R}(M)$ um elemento idempotente e seja $f=i d_{M}-e$. Observamos que $f^{2}(m)=f(f(m))=f(m-e(m))=m-e(m)-e(m)+e^{2}(m)=m-e(m)=f(m)$. Além disso $f e(m)=f(e(m))=e(m)-e^{2}(m)=0$. Então $e, f$ são idempotentes ortogonais e, pela Proposição 2.5.3(2), $M=M_{1} \bigoplus M_{2}$, em que $M_{1}=e(M)$ e $M_{2}=f(M)$. Se $e \neq 0$ e $e \neq i d_{M}$, teríamos $M_{1} \neq 0, M_{2} \neq 0$ e, portanto, $M$ não seria indecomponível. Reciprocamente, se $M$ não fosse indecomponível, existiriam submódulos $M_{1} \neq 0, M_{2} \neq 0$ tais que $M=M_{1} \bigoplus M_{2}$. Pela Proposição 2.5.3(1), teríamos idempotentes $e, f \in \operatorname{End}_{R}(M)$ tais que $e \neq 0, f \neq 0$ e $e+f=i d_{M}$. 
Em um anel local $R$ os únicos idempotentes são 0 e $1_{R}$, uma vez que se $0 \neq e \in R$ é um idempotente, temos que $e^{2}=e \Rightarrow e\left(1_{R}-e\right)=0$. Se $e \neq 1_{R}$, então $e$ e $1_{R}-e$ seriam não inversíveis e, como $R$ é local, $1_{R}=\left(1_{R}-e\right)+e$ seria não inversível. Mas $1_{R}$ é uma unidade. Portanto $e=1_{R}$.

Então se $M$ é um $R$-módulo para o qual $\operatorname{End}_{R}(M)$ é local, então $M$ é indecomponível, pela Proposição 2.5.4. Alguns autores denominam fortemente indecomponível todo $R$-módulo $M$ para o qual $\operatorname{End}_{R}(M)$ é um anel local.

Proposição 2.5.5. Para qualquer $\mathbf{p} \in k^{\mathrm{I}}$ temos:

1. $P(\mathbf{p})$ é indecomponível;

2. $O$ A-módulo top $(P(\mathbf{p}))=L(\mathbf{p})$ é simples com $\operatorname{dim}_{k} L(\mathbf{p})_{\mathbf{p}}=1$, e $L(\mathbf{p})$ é o único (a menos de isomorfismo) módulo simples com esta propriedade.

Demonstração. 1. Pelo isomorfismo natural 2.1 temos que $\operatorname{Hom}_{\mathfrak{W}}(P(\mathbf{p}), P(\mathbf{p})) \cong(P(\mathbf{p}))_{\mathbf{p}}$. Ou seja, $\operatorname{End}_{\mathfrak{W}}(P(\mathbf{p})) \cong k$. Como $k$ é um anel local, os únicos idempotentes de $\operatorname{End}_{\mathfrak{W}}(P(\mathbf{p}))$ são 0 e $i d_{P(\mathbf{p})}$. Portanto, pela Proposição 2.5.4, $P(\mathbf{p})$ é um $A$-módulo indecomponível.

2. Como $P(\mathbf{p})$ é gerado por $P(\mathbf{p})_{\mathbf{p}}$ como $A$-módulo, qualquer submódulo próprio de $P(\mathbf{p})$ não intercepta $P(\mathbf{p})_{\mathbf{p}}$. Ou seja, $N^{\prime}$ é um submódulo próprio de $P(\mathbf{p})$ se, e somente se, $\mathbf{p} \notin \operatorname{supp}\left(N^{\prime}\right)$. Como $\operatorname{supp}\left(N_{1} \bigoplus N_{2}\right)=\operatorname{supp}\left(N_{1}\right) \cup \operatorname{supp}\left(N_{2}\right)$, a soma de todos os submódulos próprios de $P(\mathbf{p})$ é um submódulo próprio. Seja $N$ a soma de todos os submódulos próprios de $P(\mathbf{p})$. Temos que $N$ é o único submódulo maximal de $P(\mathbf{p})$, pois se $N \subseteq N^{\prime} \subsetneq P(\mathbf{p})$, ou seja, $N^{\prime}$ é próprio, então $N^{\prime} \subseteq N$, resultando em $N^{\prime}=N$, e se existisse outro submódulo maximal de $P(\mathbf{p})$, este estaria contido em $N$, logo seria o próprio $N$. Assim $\operatorname{rad}(P(\mathbf{p}))=N$. Dessa forma $L(\mathbf{p})=\operatorname{top}(P(\mathbf{p}))=P(\mathbf{p}) / N$ é simples. Além disso, $L(\mathbf{p})_{\mathbf{p}}=P(\mathbf{p})_{\mathbf{p}} / N_{\mathbf{p}}=P(\mathbf{p})_{\mathbf{p}} /\{0\}=P(\mathbf{p})_{\mathbf{p}}$, implicando que $\operatorname{dim}_{k} L(\mathbf{p})_{\mathbf{p}}=1$. Para finalizar vamos provar que $L(\mathbf{p})$ é o único módulo simples com esta propriedade. Suponhamos que $S$ seja um $A$-módulo simples tal que $\operatorname{dim}_{k} S_{\mathbf{p}}=1$. Então existe $0 \neq v \in S$ tal que $A \cdot v=S$, visto que $S$ é simples. Podemos definir o $A$-epimorfismo $\varphi: P(\mathbf{p}) \rightarrow S$, por $1 \otimes 1_{k_{\mathrm{p}}} \mapsto v$. Assim $P(\mathbf{p}) / \operatorname{ker}(\varphi) \cong S$. Pela simplicidade de $S$ temos que $\operatorname{ker}(\varphi)$ é maximal, ou seja, $\operatorname{ker}(\varphi)=N$. Portanto $L(\mathbf{p})=$ $P(\mathbf{p}) / N \cong S$.

Vamos mostrar que $P(\mathbf{p})$ é cobertura projetiva de $L(\mathbf{p})$.

Definição 2.5.9. Seja $M$ um R-módulo. Uma cobertura projetiva de $M$ é um par $(P, p)$, em que $P$ é um módulo projetivo e $p: P \rightarrow M$ é um epimorfismo, tal que se $Q$ é qualquer outro módulo projetivo e $q: Q \rightarrow M$ é um epimorfismo, então existe um epimorfismo $\lambda: Q \rightarrow P$ que faz o seguinte diagrama comutar:

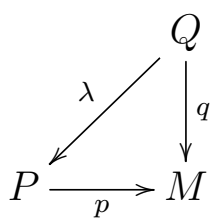

Em outras palavras $p: P \rightarrow M$ é um epimorfismo essencial, ou seja, seu kernel é um submódulo supérfluo em $P$ (um submódulo $S \subseteq P$ é supérfluo se $S+L=P$, para algum submódulo $L$ de $P$, implica que $L=P$ ). 
Proposição 2.5.6. Se $P$ é um $R$-módulo projetivo então a aplicação quociente $p: P \rightarrow$ $P / \operatorname{rad}(P)$ é uma cobertura projetiva.

Demonstração. Seja $Q$ um outro $R$-módulo projetivo e $q: Q \rightarrow P / \operatorname{rad}(P)$ outro epimorfismo, ou seja,

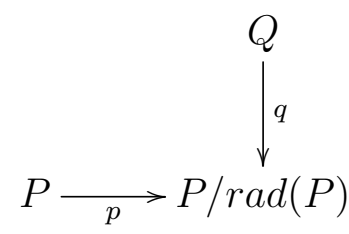

Como $p$ é epimorfismo e $Q$ é projetivo, existe $t: Q \rightarrow P$ com $p t=q$. Assim, $t(Q)+$ $\operatorname{rad}(P)=P$. Agora, se $t(Q) \neq P$, existe um submódulo maximal $X \subsetneq P \operatorname{com} t(Q) \subseteq X$. Mas $\operatorname{rad}(P) \subseteq X$, pois o radical é a intersecção de todos os submódulos maximais; logo $P=t(Q)+\operatorname{rad}(P) \subseteq X \subsetneq P$, uma contradição. Então $t(Q)=P$. Portanto $t$ é epimorfismo, como desejado.

Sabemos que $P(\mathbf{p})$ é $A$-módulo projetivo e que existe um epimorfismo $p: P(\mathbf{p}) \rightarrow L(\mathbf{p})$. Portanto, usando a Proposição 2.5.6, $P(\mathbf{p})$ é uma cobertura projetiva de $L(\mathbf{p})$.

\subsection{Involução e dualidade restrita}

Basearemos essa seção, principalmente, em [FGM14][Seção 3.3].

Seja $R$ uma $k$-álgebra. Um anti-automorfismo involutivo de $R$ é um isomorfismo $\tau: R \rightarrow$ $R$, tal que $\tau^{2}=\tau$ e $\tau(a b)=\tau(b) \tau(a)$, para todos $a, b \in R$. A álgebra de Weyl de posto infinito $A=A_{k, \text { I }}$ possui um anti-automorfismo involutivo canônico $\diamond: A \rightarrow A$ que é definido nos geradores via $X_{i}^{\diamond}=Y_{i}$. Este anti-automorfismo involutivo fixa a $k$-álgebra comutativa $A_{0}$. De fato, seja $t_{i}$, com $i \in \mathrm{I}$ :

$$
t_{i}^{\diamond}=\left(X_{i} Y_{i}\right)^{\diamond}=Y_{i}^{\diamond} X_{i}^{\diamond}=\left(X_{i}^{\diamond}\right)^{\diamond} Y_{i}=X_{i} Y_{i}=t_{i}
$$

É interessante notar que, se $N$ é um $A$-módulo à direita então podemos definir um $A$ módulo à esquerda $N^{t}$; basta considerar $N^{t}=N$, como grupos abelianos e definir a ação de $A$ pela esquerda por $a n=n a^{\diamond}$, para todos $a \in A, n \in N^{t}$. Para verificar que $N^{t}$ é um $A$-módulo à esquerda, basta verificar $(a b) n=a(b n)$ :

$$
(a b) n=n(a b)^{\diamond}=n\left(b^{\diamond} a^{\diamond}\right)=\left(n b^{\diamond}\right) a^{\diamond}=a\left(n b^{\diamond}\right)=a(b n)
$$

em que $a, b \in A$ e $n \in N^{t}$. A mesma construção pode ser usada para transformar módulos à esquerda em módulos à direita.

Vamos definir o primeiro dual de um $R$-módulo. Seja $M$ um $R$-módulo à direita, o primeiro dual $M^{*}=\operatorname{Hom}_{R}(M, R)$ é um $R$-módulo à esquerda com ação definida por $(r f)(a)=f(a r)$, em que $r \in R, f \in M^{*}$ e $a \in M$. Assim, $M^{*}$ é um $R$-módulo de tipo oposto ao tipo de $M$, ou seja, os elementos de $R$ agem no lado oposto ao que agem em $M$.

Se $f: M \rightarrow N$ é um $R$-homomorfismo, então $f^{*}: N^{*} \rightarrow M^{*}$ é definido por $\varphi \mapsto \varphi f$. Se $f$ for um epimorfismo, então $f^{*}$ é um monomorfismo. Suponha que $\varphi \in N^{*}$ e $f^{*}(\varphi)=0$. Assim $\varphi f=0$ é o homomorfismo nulo de $M$ para $R$, ou seja, $\varphi(f(m))=0$, para todo $m \in$ $M$. Como $f$ é epimorfismo, segue que $\varphi(n)=0$, para todo $n \in N$, portanto $\varphi=0$.

Então, o primeiro dual converte sobrejetividade em injetividade. Suponhamos agora que $f: M \rightarrow N$ seja um monomorfismo. Então $M$ é identificado com um submódulo de $N$, $M \cong f(M) \subseteq N$. Para $\varphi \in N^{*}$, o homomorfismo $f^{*}(\varphi)=\varphi f$ é simplesmente a restrição 
de $\varphi$ ao submódulo $f(M)$. Dizer que $f^{*}$ é epimorfismo significa que todo homomorfismo $\phi: M \rightarrow R$ tem a forma $\varphi f$, isto é, cada homomorfismo $f(M) \rightarrow R$ pode ser estendido a um homomorfismo $N \rightarrow R$. Isto nem sempre é verdade. Porém se $k$ é um corpo e $M$ e $N$ são $k$-espaços vetoriais de dimensão finita (vale para dimensão infinita também, mas a justificativa utiliza o Lema de Zorn, e por isso não é construtiva) em que $f: M \rightarrow N$ é uma aplicação linear injetora, então $f^{*}$ é sobrejetora. De fato, $M$ é identificado com o subespaço $f(M)$ de $N$, isto é, $M \cong f(M)$; pelo Teorema do completamento da base, existe complemento para $f(M)$ em $N$, isto é, existe $P$ tal que $N=f(M) \oplus P$; seja $\pi: N \rightarrow M$ a projeção canônica de $N$ em $f(M)$, isto é, $\pi(f(m)+p)=m$, para todos $m \in M, p \in P$, ou seja, $\pi f=i d_{M}$; logo $f^{*} \pi^{*}=i d_{M^{*}}$; portanto, para cada $\varphi$ em $M^{*}$, temos que $f^{*}\left(\pi^{*}(\varphi)\right)=\varphi$, em que $\pi^{*}(\varphi)=\varphi \pi \in N^{*}$, então $f^{*}$ é uma aplicação linear sobrejetora.

O próximo resultado que vamos mostrar, chamado de "Lema para produzir Injetivos", nos permite utilizar módulos injetivos conhecidos sobre um anel para produzir módulos injetivos sobre outro. Sejam $S, R$ anéis e $P$ um $(R, S)$-bimódulo fixo que é plano como $R$ módulo à esquerda. Lembremos que um $R$-módulo à esquerda $P$ é dito ser plano se o funtor $-\otimes_{R} P$ é exato na categoria dos $R$-módulos à direita. Para qualquer $M \in \bmod -S$, definimos $\tilde{M}=\operatorname{Hom}_{S}\left(P_{S}, M_{S}\right)$.

Podemos tomar $\tilde{M}$ como um $R$-módulo à direita por: $(f r)(p)=f(r p)$, em que $f \in \tilde{M}$, $r \in R$ e $p \in P$.

Lema 2.6.1. Se $M$ é um $S$-módulo à direita injetivo, então $\tilde{M}$ será um R-módulo à direita injetivo.

Demonstração. Precisamos provar a exatidão do funtor $\operatorname{Hom}_{R}(-, \tilde{M})$. Para qualquer $N \in$ $\bmod -R$, temos o seguinte isomorfismo canônico (Teorema B.3.21, Apêndice B):

$$
\operatorname{Hom}_{R}(N, \tilde{M})=\operatorname{Hom}_{R}\left(N, \operatorname{Hom}_{S}(P, M)\right) \cong \operatorname{Hom}_{S}\left(N \otimes_{R} P, M\right)
$$

Como ${ }_{R} P$ é plano, o funtor $-\otimes_{R} P$ é exato em $\bmod -R$, para qualquer $N \in \bmod -R$. Como $M_{S}$ é injetivo, então $\operatorname{Hom}_{S}(-, M)$ é exato em mod- $S$. Concluímos do isomorfismo acima que o funtor $\operatorname{Hom}_{R}(-, \tilde{M})$ é exato em $\bmod -R$. Portanto $\tilde{M}$ é um $R$-módulo à direita injetivo.

Observamos que um $R$-módulo à esquerda $M$, tal que $R$ é uma $k$-álgebra, pode ser visto como $(R, k)$-bimódulo e qualquer $k$-espaço vetorial é certamente $k$-injetivo. Então, temos o seguinte caso especial do Lema 2.6.1:

Corolário 2.6.1. Seja $R$ uma k-álgebra. Seja ${ }_{R} P$ um $R$-módulo à esquerda projetivo fixo, visto como $(R, k)$-bimódulo. Então, para qualquer $k$-espaço vetorial $M, \operatorname{Hom}_{k}(P, M)$ é um $R$-módulo à direita injetivo.

Demonstração. Todo módulo livre é plano; e uma soma direta de módulos é plana se, e somente se, cada módulo é plano. Como todo módulo projetivo é somando direto de um módulo livre, temos que todo módulo projetivo é plano (ver [Rot09], Capítulo 3, Proposição 3.46, pág. 132). Então o $R$-módulo à esquerda $P$ é plano, ou seja, o funtor $-\otimes_{R} P$ é exato. Pelo Lema 2.6.1, $\operatorname{Hom}_{R}(-, \tilde{M})$ é exato em $\bmod -R$. Portanto $\tilde{M}=\operatorname{Hom}_{k}(P, M)$ é um $R-$ módulo à direita injetivo.

A álgebra de Weyl de posto infinito $A=A_{k, \mathrm{I}}$ é uma $k$-álgebra e $P(\mathbf{p})$ é um $A$-módulo de peso à esquerda projetivo. Então $P(\mathbf{p})$ tem estrutura de $(A, k)$-bimódulo. Pelo Corolário 2.6.1 temos que, em particular, $\operatorname{Hom}_{k}(P(\mathbf{p}), k)$ é um $A$-módulo à direita injetivo, ou seja, $P(\mathbf{p})^{*}=\operatorname{Hom}_{k}(P(\mathbf{p}), k)$ é um $A$-módulo à direita injetivo. 
Denotaremos por $\mathfrak{W}^{f}$ a subcategoria plena de $\mathfrak{W}$ consistindo de todos os $A$-módulos de peso com espaços de peso de dimensão finita. Definiremos uma dualidade restrita na categoria $\mathfrak{W}^{f}$. Esta dualidade preserva objetos simples e envia objetos projetivos em injetivos.

Pela Subseção 2.5 temos que $P(\mathbf{p})$ e $L(\mathbf{p})$ pertencem à $\mathfrak{W}^{f}$, para todo $\mathbf{p} \in k^{\mathrm{I}}$.

Para uma $k$-álgebra $R$ definimos a álgebra oposta $R^{o p}$ de $R$ como a $k$-álgebra cuja estrutura de espaço vetorial do conjunto subjacente é a mesma que em $R$, mas a multiplicação $\circ$ em $R^{o p}$ é definida pela fórmula $a \circ b=b a$. Podemos observar que qualquer $R$-módulo à esquerda pode ser visto como um $R^{o p}$-módulo à direita e vice versa. Seja $N$ um $R$-módulo à esquerda. Vamos definir a ação de $R^{o p}$ à direita via $n \cdot a=$ an para $a \in R^{o p}$ e $n \in N$. Vejamos que $N$ é $R^{o p}$-módulo à direita:

$$
n \cdot(a \circ b)=n \cdot(b a)=(b a) n=b(a n)=(a n) \cdot b=(n \cdot a) \cdot b
$$

para todos $a, b \in R^{o p}$ e $n \in N$. Dessa maneira a categoria mod- $R^{o p}$ é identificada com a categoria $R$-mod.

O conceito de dualidade é muito amplo e geral. A ideia comum é que há duas coisas que basicamente são dois lados da mesma moeda. Do ponto de vista da teoria de categorias, a dualidade pode ser vista como um funtor. O protótipo para a nossa teoria da dualidade é a teoria dos espaços vetoriais de dimensão finita. Esta teoria não se estende para espaços em que a dimensão é arbitrária.

Vejamos um exemplo que foi retirado de [ASS06], Capítulo $I$.

Seja $R$ uma $k$-álgebra com dimensão finita. Sejam modfg- $R$, a categoria dos $R$-módulos à direita finitamente gerados, e modfg- $R^{o p}$, a categoria dos $R^{o p}$-módulos à direita (ou dos $R$ módulos à esquerda) finitamente gerados. Um $R$-módulo à direita $M$ é um $k$-espaço vetorial de dimensão finita. Podemos definir o funtor $D: \operatorname{modfg}-R \longrightarrow \operatorname{modfg}-R^{o p}$, que associa cada $R$-módulo à direita $M$ com o seu primeiro dual $M^{*}=\operatorname{Hom}_{k}(M, k)$, que como já vimos, é um $R$-módulo à esquerda. Como $M$ é um $k$-espaço vetorial, $M^{*}$ é o $k$-espaço dual. Para um homomorfismo $h: M \rightarrow N$ em modfg- $R$ temos o homomorfismo dual de $R$-módulos à esquerda $D(h)=\operatorname{Hom}_{k}(h, k)=h^{*}: D(N) \rightarrow D(M)$, definido por $\varphi \mapsto \varphi h$.

Lembremos que um funtor covariante $F: \mathcal{C} \rightarrow \mathcal{D}$ é dito uma equivalência entre as categorias $\mathcal{C}$ e $\mathcal{D}$, se existe um funtor $G: \mathcal{D} \rightarrow \mathcal{C}$ e isomorfismos funtoriais $\Psi: 1_{\mathcal{C}} \stackrel{\simeq}{\rightarrow} G F$ e $\Phi: 1_{\mathcal{D}} \stackrel{\sim}{\rightarrow} F G$, tais que $1_{\mathcal{C}}$ e $1_{\mathcal{D}}$ são os funtores identidade em $\mathcal{C}$ e $\mathcal{D}$, respectivamente. Neste caso dizemos que o funtor $G$ é um quase-inverso de $F$ e que as categorias $\mathcal{C}$ e $\mathcal{D}$ são equivalentes $(\mathcal{C} \approx \mathcal{D})$. Um funtor contravariante $F: \mathcal{C} \rightarrow \mathcal{D}$ é dito uma dualidade entre as categorias $\mathcal{C}$ e $\mathcal{D}$ se o funtor covariante induzido $F^{\prime}: \mathcal{C}^{o p} \rightarrow \mathcal{D}$ for uma equivalência entre as categorias $\mathcal{C}^{o p}$ e $\mathcal{D}$.

O funtor $D$ do exemplo, é uma dualidade de categorias chamado de $k$-dualidade padrão (para mais detalhes veja [ASS06], Capítulo $I$ ).

Voltemos agora nossa atenção para a categoria $\mathfrak{W}^{f}$. Aqui é importante ressaltar que considerar o primeiro dual do módulo pode trazer problemas, como não existir a equivalência de categorias. Sendo assim vamos considerar uma dualidade restrita.

A categoria $\mathfrak{W}^{f}$ tem uma dualidade restrita canônica $\vee$ definida como segue: para $M \in$ $\mathfrak{W}^{f}$ temos que

$$
M^{\vee}:=\bigoplus_{\mathbf{p} \in k^{\mathrm{I}}} \operatorname{Hom}_{k}\left(M_{\mathbf{p}}, k\right)
$$

Este tipo de dualidade restrita também é conhecida como contragradiente.

Observamos que cada espaço de peso $M_{\mathbf{p}}$ tem estrutura de $\left(A_{0}, k\right)$-bimódulo, então $\operatorname{Hom}_{k}\left(M_{\mathbf{p}}, k\right)=M_{\mathbf{p}}^{*}$ é um $A_{0}$-módulo à direita. Logo $M^{\vee}=\bigoplus_{\mathbf{p} \in k^{\mathrm{I}}} M_{\mathbf{p}}^{*}$ é um $A_{0}$-módulo 
à direita.

A ação da $k$-álgebra de Weyl $A$ pela esquerda em $M^{\vee}$ é definida via o anti-automorfismo involutivo $\diamond$, definido no início dessa seção. Para $i \in \mathrm{I}$, sejam $X_{i}, Y_{i} \in A$ :

$$
Y_{i} \cdot \tau(v)=\tau\left(Y_{i}^{\diamond} \cdot v\right)=\tau\left(X_{i} v\right)
$$

e

$$
X_{i} \cdot \tau(v)=\tau\left(X_{i}^{\diamond} \cdot v\right)=\tau\left(Y_{i} v\right)
$$

para quaisquer $v \in M, \tau \in M^{\vee}$. Para verificar que $M^{\vee}$ é um $A$-módulo à esquerda com essa ação basta verificar que $(a b) \tau=a(b \tau)$, para todos $a, b \in A$ e $\tau \in M^{\vee}$. De fato:

$$
\left.(a b) \tau(m)=\tau\left((a b)^{\diamond} m\right)=\tau\left(\left(b^{\diamond} a^{\diamond}\right) m\right)=\tau\left(b^{\diamond}\left(a^{\diamond} m\right)\right)=b \tau\left(a^{\diamond} m\right)\right)=a(b \tau(m))
$$

para todo $m \in M$, ou seja, $(a b) \tau=a(b \tau)$.

Seja $M \in \mathfrak{W}^{f}$, ou seja, $M=\bigoplus_{\mathbf{p} \in k^{\mathrm{I}}} M_{\mathbf{p}}$, em que $M_{\mathbf{p}}$ tem dimensão finita sobre $k$, para todo $\mathbf{p} \in k^{\mathrm{I}}$. Logo $M_{\mathbf{p}}^{*}=\operatorname{Hom}_{k}\left(M_{\mathbf{p}}, k\right)$ também tem dimensão finita sobre $k$, para todo $\mathbf{p} \in$ $k^{\mathrm{I}}$. Seja $\tau \in M_{\mathbf{p}}^{*}$ e $\mathfrak{m}_{\mathbf{p}}$ um ideal maximal em $A_{0}$. Para todos $i \in \mathrm{I}, m \in M_{\mathbf{p}}$ temos que:

$$
\left(t_{i}-p_{i}\right) \tau(m)=\tau\left(\left(t_{i}-p_{i}\right)^{\diamond} m\right)=\tau\left(\left(t_{i}-p_{i}\right) m\right)=\tau(0)=0,
$$

pois $\diamond$ fixa os elementos de $A_{0}$. Logo $\mathfrak{m}_{\mathbf{p}} \tau=0$. Então $M_{\mathbf{p}}^{*}$ é um espaço de peso. Assim $M^{\vee}=\bigoplus_{\mathbf{p} \in k^{\mathrm{I}}} M_{\mathbf{p}}^{*}$ é um $A$-módulo de peso, tal que $M_{\mathbf{p}}^{*}$ tem dimensão finita sobre $k$, para todo $\mathbf{p} \in k^{\mathrm{I}}$. Portanto $M^{\vee} \in \mathfrak{W}^{f}$. Ou seja, a dualidade restrita $\vee$ envia $M \in \mathfrak{W}^{f}$ em $M^{\vee} \in$ $\mathfrak{W}^{f}$.

Se $f: M \rightarrow N$ é um homomorfismo em $\mathfrak{W}^{f}$, então $f^{\vee}: N^{\vee} \rightarrow M^{\vee}$, definido por $\varphi \mapsto \varphi f$, é um homomorfismo em $\mathfrak{W}^{f}$.

Temos que $\operatorname{supp}\left(M^{\vee}\right)=\operatorname{supp}(M)$, para todo $M \in \mathfrak{W}^{f}$. De fato, $M_{\mathbf{p}} \neq\{0\}$ para algum $\mathbf{p}$ $\in k^{\mathrm{I}}$ se, e somente se, existe $0 \neq x \in M_{\mathbf{p}}$ tal que podemos definir uma transformação linear não nula de $M_{\mathbf{p}}$ em $k, x \mapsto 1$. Em outras palavras $M_{\mathbf{p}} \neq\{0\}$ se, e somente se, $M_{\mathbf{p}}^{*} \neq\{0\}$, e segue o resultado.

Temos que $\operatorname{supp}(P(\mathbf{p}))=\operatorname{supp}\left(P(\mathbf{p})^{\vee}\right)$. Seja $N^{\vee}$ a soma de todos os submódulos próprios de $P(\mathbf{p})^{\vee}$; então $N^{\vee}$ é o único submódulo maximal de $P(\mathbf{p})^{\vee}$, assim $L(\mathbf{p})^{\vee}=P(\mathbf{p})^{\vee} / N^{\vee}$ é um $A$-módulo simples. Temos também que $\operatorname{supp}\left(L(\mathbf{p})^{\vee}\right)=\operatorname{supp}(L(\mathbf{p}))$, e como $\operatorname{dim}_{k} L(\mathbf{p})_{\mathbf{p}}=1$, existe $0 \neq u \in L(\mathbf{p})_{\mathbf{p}}$. Logo $\mathbf{p} \in \operatorname{supp}(L(\mathbf{p}))=\operatorname{supp}\left(L(\mathbf{p})^{\vee}\right)$, ou seja, existe $0 \neq \tau \in$ $L(\mathbf{p})_{\mathbf{p}}^{*}$. Como $L(\mathbf{p})^{\vee}$ é um $A$-módulo simples, ele é gerado por $\tau$. Existe um epimorfismo $\varphi: P(\mathbf{p}) \rightarrow L(\mathbf{p})^{\vee}$, definido por $1 \otimes 1_{k_{\mathbf{p}}} \mapsto \tau$, ou seja, $L(\mathbf{p})^{\vee}$ é imagem homomórfica de $P(\mathbf{p})$. Então $P(\mathbf{p}) / \operatorname{ker}(\varphi) \cong L(\mathbf{p})^{\vee}$, e pela simplicidade de $L(\mathbf{p})^{\vee}, \operatorname{ker}(\varphi)$ é um submódulo maximal em $P(\mathbf{p})$. Na demostração da Proposição 2.5.5(2) vimos que existe um único submódulo maximal $N$ em $P(\mathbf{p})$, portanto, $L(\mathbf{p}) \cong L(\mathbf{p})^{\vee}$.

Agora vamos definir um envelope injetivo, que também é conhecido como casca injetiva. Antes disso, precisamos de outras definições e resultados. Para mais detalhes, indicamos [Lam98].

Definição 2.6.1. Sejam $R$ um anel e $M$ um $R$-módulo à esquerda. Um $R$-módulo à esquerda $E \supseteq M$ é dito ser uma extensão essencial de $M$ se cada submódulo não nulo de E intercepta $M$ não trivialmente. Uma extensão essencial $E \supseteq M$ é dita ser maximal se nenhum módulo contendo E propriamente é uma extensão essencial de $M$.

Se $E \supseteq M$ é uma extensão essencial, dizemos também que $M$ é um submódulo essencial (ou grande) de $E$ e denotamos $M \subseteq_{e} E$. A noção de um submódulo grande é dual à noção 
de um submódulo supérfluo (ou pequeno). Observamos essa dualidade pelas definições: Um submódulo $S \subseteq P$ é supérfluo se $S+L=P$, para algum submódulo $L$ de $P$, implica $L=P$, enquanto que o submódulo $S \subseteq E$ é essencial se $S \cap L=\{0\}$, para algum submódulo $L$ de $E$, implica $L=\{0\}$.

Observação 2.6.1. Temos o seguinte fato: $M \subseteq_{e} E$ se, e somente se, para cada $0 \neq a \in$ $E$, existe $r \in R$ tal que $0 \neq r a \in M$. Esse é um fato bastante óbvio que dificilmente requer uma prova. No entanto, ele fornece uma forma conveniente para verificar se $M \subseteq_{e} E$. Na verdade, na maioria das vezes, nós verificamos essencialmente por aplicação deste critério.

Observação 2.6.2. (Transitividade) $S e M \subseteq_{e} E$ e $E \subseteq_{e} E^{\prime}$, então $M \subseteq_{e} E^{\prime}$. Isto segue facilmente da Observação 2.6.1, ou diretamente da Definição 2.6.1. A propriedade da transitividade para essencialidade é considerada básica.

A noção de uma extensão essencial leva a uma nova interpretação da injetividade de um $R$-módulo, como segue.

Lema 2.6.2. Um R-módulo à esquerda $M$ é injetivo se, e somente se, ele não tem nenhuma extensão essencial própria.

Demonstração. Primeiro vamos assumir que $M$ é injetivo, e considere uma extensão própria qualquer $E \supsetneq M$. Temos a seguinte sequência exata $0 \longrightarrow M \stackrel{\iota}{\longrightarrow} E \stackrel{\pi}{\longrightarrow} E / M \longrightarrow 0$. Pelo Teorema B.3.13 (Apêndice B) essa sequência cinde, então temos que $E=M \bigoplus N$, para algum submódulo $N \neq\{0\}$ (pois $E \supsetneq M$ ). Como $N \cap M=\{0\}$, então $E \supseteq M$ não é uma extensão essencial. Reciprocamente, vamos assumir que $M$ não tem nenhuma extensão essencial própria. Pelo Teorema B.3.16 (Apêndice B), $M$ pode ser identificado com um submódulo de um $R$-módulo à esquerda injetivo $I$, isto é, existe um monomorfismo $\iota: M \rightarrow I$. Pelo Lema de Zorn, existe um submódulo $S \subseteq I$, maximal com respeito a propriedade $S \cap M=\{0\}$. Então no quociente $I / S$, qualquer submódulo não nulo $S^{\prime} / S$ intercepta a imagem de $M$ não trivialmente, então $\iota(M) \subseteq_{e} I / S$. Por hipótese, devemos ter $\iota(M)=I / S$. Isto significa que $I=M \oplus S$. Então, pelo Corolário B.3.3 (Apêndice B), $M$ é um $R$-módulo à esquerda injetivo.

Lema 2.6.3. Qualquer $R$-módulo à esquerda $M$ tem uma extensão essencial maximal.

Demonstração. Vamos fixar o módulo injetivo $I \supseteq M$, e considerar qualquer família de extensões essenciais de $M$ em $I$ que são linearmente ordenadas por inclusão. Pela Observação 2.6.1, é claro que a união da família é também uma extensão essencial de $M$. Pelo Lema de Zorn, segue que podemos encontrar um submódulo $E$ maximal com respeito a propriedade $M \subseteq_{e} E \subseteq I$. Afirmamos que $E$ é uma extensão essencial maximal de $M$. De fato, se isto é falso, podemos encontrar um módulo $E^{\prime}$ contendo $E$ propriamente, ou seja, $E \subsetneq E^{\prime}$ tal que $M \subseteq_{e} E^{\prime}$ ( $E^{\prime}$ é algum $R$-módulo, que não precisa necessariamente estar em $I$ ). Pela injetividade de $I$, a aplicação inclusão $E \subseteq I$ pode ser estendida para alguma $g: E^{\prime} \rightarrow I$. Temos que $(k e r(g)) \cap M=\{0\}$ e como $M \subseteq \subseteq_{e} E^{\prime}$ implica que $\operatorname{ker}(g)=\{0\}$. Logo, podemos identificar $E^{\prime}$ com $g\left(E^{\prime}\right)$. Mas então $M \subseteq_{e} E^{\prime}$ contradiz a maximalidade de $E$.

Teorema 2.6.1. Para $R$-módulos à esquerda $M \subseteq I$, as seguintes afirmações são equivalentes:

1. I é extensão essencial maximal sobre $M$.

2. I é injetivo, e é essencial sobre $M$. 


\section{I é injetivo minimal sobre $M$.}

Demonstração. Vamos mostrar que 1. $\Longrightarrow$ 2. Como $I$ já é uma extensão essencial, basta mostrar que $I$ é injetivo. Pela Propriedade da Transitividade na Observação 2.6.2, 1. implica que $I$ não tem extensão essencial própria, pois caso $I \subsetneq_{e} E^{\prime}$, então $M \subseteq_{e} E^{\prime}$, contrariando a maximalidade de $I$. Portanto, $I$ é injetivo pelo Lema 2.6.2.

Agora vamos mostrar que 2. $\Longrightarrow$ 3. Basta mostrar que $I$ é minimal. Seja $I^{\prime}$ um módulo injetivo tal que $M \subseteq I^{\prime} \subseteq I$. Temos a seguinte sequência exata $0 \longrightarrow I^{\prime} \stackrel{\iota}{\longrightarrow} I \stackrel{\pi}{\longrightarrow} I / I^{\prime} \longrightarrow 0$. Pelo Teorema B.3.13 (Apêndice B) essa sequência cinde, então temos que $I=I^{\prime} \bigoplus N$, para algum submódulo $N \subseteq I$. Como $N \cap M=\{0\}$, devemos ter $N=\{0\}$ (pois $M \subseteq e I$ ), então $I^{\prime}=I$.

3. $\Longrightarrow 1$. Vamos assumir que $I$ é injetivo minimal sobre $M$. A prova do Lema 2.6.3 produz um submódulo $E \subseteq I$ que é uma extensão essencial maximal sobre $M$. Usando $1 . \Longrightarrow 2 \mathrm{em}$ $E$, concluímos que $E$ é injetivo. A minimalidade de $I$ implica que $E=I$, como desejado.

Definição 2.6.2. Se os R-módulos à esquerda $M \subseteq I$ satisfazem as propriedades 1., 2. e 3. do Teorema 2.6.1 (que são equivalentes), dizemos que I é um envelope injetivo (ou casca injetiva) de $M$.

Em outras palavras um envelope injetivo para um $R$-módulo $M$ é qualquer módulo injetivo $I$ que seja uma extensão essencial de $M$, ou seja, é o menor módulo injetivo contendo $M$.

Observamos que, pelo Lema 2.6.3, qualquer módulo $M$ tem um envelope injetivo.

Os módulos injetivos são duais dos módulos projetivos, pois em termos dos diagramas que caracterizam os dois, o diagrama da injetividade é o diagrama da projetividade com as flechas invertidas. Temos também que a noção de submódulo essencial é dual a noção de submódulo supérfluo. Logo a cobertura projetiva é o dual do envelope injetivo.

Como $P(\mathbf{p})$ é $A$-módulo projetivo, temos que $P(\mathbf{p})^{\vee}$ é $A$-módulo injetivo.

Como $p: P(\mathbf{p}) \rightarrow P(\mathbf{p}) / N=L(\mathbf{p})$ é um epimorfismo, temos que $p^{\vee}: L(\mathbf{p})^{\vee} \rightarrow P(\mathbf{p})^{\vee}$ é um monomorfismo.

Na Seção 2.5, com $p: P(\mathbf{p}) \rightarrow L(\mathbf{p})$, provamos que $(P(\mathbf{p}), p)$ é uma cobertura projetiva de $L(\mathbf{p})$. Então por conta da dualidade $\vee$, temos que $\left(P(\mathbf{p})^{\vee}, p^{\vee}\right)$ é o envelope injetivo de $L(\mathbf{p})^{\vee} \cong L(\mathbf{p})$.

\subsection{Realização via ação polinomial}

Realização matemática refere-se a um processo no qual um objeto abstrato e genérico é transformado em um objeto exato e específico. Após este processo, nós dizemos que o objeto abstrato está matematicamente realizado. Vamos tomar os $A$-módulos de peso projetivos $P(\mathbf{p})$ e através da realização via ação polinomial transformá-los em objetos concretos.

Esta seção será baseada em [FGM14][Seção 3.4].

Vamos lembrar que no Capítulo 1, Seção 1.3, definimos $B$ como a $k$-álgebra comutativa dos polinômios em infinitas variáveis $x_{i}, i \in \mathrm{I}$. Podemos tomar $B$ como um $A$-módulo à esquerda, em que a ação de $X_{i}$ em $B$ é dada pela multiplicação pela variável $x_{i}$, enquanto $Y_{i}$ age pela diferenciação com respeito à $x_{i}$.

Essa ação de $A=A_{k, \mathrm{I}}$ em $B$ admite a seguinte generalização óbvia. Para $\mathbf{p} \in k^{\mathrm{I}}$, seja $\mathbf{x}^{\mathbf{p}}:=\prod_{i \in \mathrm{I}} x_{i}^{p_{i}}$. Denotaremos por $B(\mathbf{p})$ o conjunto de todas as combinações $k$-lineares finitas dos elementos $\mathbf{x}^{\mathbf{m}}$, em que $\mathbf{m} \in \mathbf{p}+\mathbb{Z}_{f}^{\mathrm{I}}$ (ou seja, $\mathbf{m}$ pertence a classe de $\mathbf{p}$ em $k^{\mathrm{I}} / \mathbb{Z}_{f}^{\mathrm{I}}$ ). Definimos a ação de $X_{i}$ em $B(\mathbf{p})$ à esquerda, pela multiplicação por $x_{i}$ e a ação de $Y_{i}$ em 
$B(\mathbf{p})$ à esquerda, pela derivada parcial com respeito à $x_{i}$. Dessa forma $B(\mathbf{p})$ é um $A$-módulo à esquerda.

Para $\mathbf{x}^{\mathbf{v}} \in B(\mathbf{p})$, ou seja, $\mathbf{v} \in \mathbf{p}+\mathbb{Z}_{f}^{\mathrm{I}}$, vamos considerar o ideal maximal $\mathfrak{m}_{\mathbf{v}}=\left\langle t_{i}-v_{i}\right\rangle_{i \in \mathrm{I}}$ de $A_{0}$. Então, para $i \in \mathrm{I}$ :

$$
\left(t_{i}-v_{i}\right)\left(\mathbf{x}^{\mathbf{v}}\right)=\left(X_{i} Y_{i}-v_{i}\right)\left(\mathbf{x}^{\mathbf{v}}\right)=v_{i} \mathbf{x}^{\mathbf{v}}-v_{i} \mathbf{x}^{\mathbf{v}}=0
$$

Então $\mathfrak{m}_{\mathbf{v}} \cdot \mathbf{x}^{\mathbf{v}}=\{0\}$. Logo, $B(\mathbf{p})$ tem estrutura de $A$-módulo de peso. Portanto,

$$
B(\mathbf{p})=\bigoplus_{\mathbf{v} \in \mathbf{p}+\mathbb{Z}_{f}^{\mathrm{I}}} B(\mathbf{p})_{\mathbf{v}}
$$

em que $\operatorname{supp}(B(\mathbf{p}))=\mathbf{p}+\mathbb{Z}_{f}^{\mathrm{I}}$ e todos os espaços de peso não nulos de $B(\mathbf{p})$ são unidimensionais.

\section{Proposição 2.7.1.}

1. Seja $\mathbf{p} \in k^{\mathrm{I}}$, tal que se $p_{i} \in \mathbb{Z}$ implica que $p_{i} \in\{0,1,2, \ldots\}$, para todo $i$. Então $B(\mathbf{p})$ e $B(\mathbf{p})^{\vee}$ são o envelope injetivo e a cobertura projetiva de $L(\mathbf{p})$, respectivamente.

2. Seja $\mathbf{p} \in k^{\mathrm{I}}$, tal que se $p_{i} \in \mathbb{Z}$ implica que $p_{i} \in\{-1,-2, \ldots\}$, para todo $i$. Então $B(\mathbf{p})$ e $B(\mathbf{p})^{\vee}$ são a cobertura projetiva e o envelope injetivo de $L(\mathbf{p})$, respectivamente.

Demonstração. Vamos provar o item 2. O item 1. é provado similarmente. Seja $\mathbf{p} \in k^{\mathrm{I}}$, tal que se $p_{i} \in \mathbb{Z}$ implica que $p_{i} \in\{-1,-2, \ldots\}$, para todo $i$. Como $\vee$ é uma dualidade, é suficiente mostrar que $B(\mathbf{p}) \cong P(\mathbf{p})$. Sabemos pelo isomorfismo natural 2.1 (Capítulo 2, Seção 2.5) que $\operatorname{Hom}_{\mathfrak{W}}(P(\mathbf{p}), B(\mathbf{p})) \cong B(\mathbf{p})_{\mathbf{p}}$. Por construção, $B(\mathbf{p})_{\mathbf{p}} \neq\{0\}$. Então existe um homomorfismo de $A$-módulos não nulo $\varphi: P(\mathbf{p}) \rightarrow B(\mathbf{p})$. Temos que $\varphi$ é um monomorfismo. Comparando os elementos de $P(\mathbf{p})$ e $B(\mathbf{p})$, segue que a aplicação $\varphi$ é um epimorfismo, ou seja, é uma bijeção.

\subsection{Referências}

A Seção 2.1 foi baseada em [Cou95][Capítulo 5]. A Seção 2.2 foi baseada em [Cou95] [Capítulo 7]. A Seção 2.3 foi baseada em [Cou95] [Capítulo 8]. A Seção 2.4 foi baseada em [FGM14] [Seção 3.1]. A Seção 2.5 foi baseada em [FGM14] [Seção 3.2]. Também utilizamos [Lam01], [Bea99] [Capítulos 2 e 3] e [Bro82] [Seção 3.3]. A Seção 2.6 foi baseada em [FGM14] [Seção 3.3] e em [Lam98] [Capítulo 3]. A Seção 2.7 foi baseada em [FGM14] [Seção 3.4]. 
64 MÓDUlOS SOBRE A ÁlGEBRA DE WEYL 


\section{Capítulo 3}

\section{Classificação}

\subsection{Módulos de peso simples sobre uma álgebra de Weyl generalizada $\mathbf{A}=\mathbf{D}(\underline{\sigma}, \underline{\mathbf{a}})$}

Antes de tudo, daremos um breve histórico dos estudos sobre módulos de peso simples sobre álgebras de Weyl.

Em [Blo81], Block classificou os módulos simples para a álgebra de Weyl $A_{1}$ sobre um corpo $k$ algebricamente fechado de característica 0 junto com os módulos simples para a álgebra de Lie $\mathfrak{s l}(2, k)$. Uma abordagem alternativa usando álgebras de Weyl generalizadas quando $k$ é arbitrário foi proposta em [Bav92a], [Bav92b] e em [BvO97].

Módulos de peso sobre $A_{1}$ (e sobre algumas outras álgebras de Weyl generalizadas de grau 1) para corpos arbitrários foram estudadas em [DGO96]. Para corpos algebricamente fechados de característica 0 , módulos simples holônomos sobre a álgebra de Weyl $A_{2}$ foram classificados em [BvO00]. Módulos de peso generalizados e de peso para $A_{n} \operatorname{com} n<\infty$ (e para algumas outras álgebras de Weyl generalizadas) sobre um corpo algebricamente fechado foram investigadas em [BBL97] e [BB00]. Em particular, [BBL97] deu uma descrição explícita dos módulos de peso para álgebras de Weyl complexas $A_{n} \operatorname{com} n<\infty$ e usou isso para construir os módulos de peso que têm todos os espaços de peso unidimensionais para álgebras de Lie complexas simples de dimensões finitas. O artigo [BB00] forneceu uma classificação das representações em blocos do tipo mansa na categoria dos módulos de peso localmente finitos e descreveu módulos indecomponíveis em blocos do tipo mansa. Neste caso, todos os módulos simples podem ser obtidos como produtos tensoriais de módulos simples sobre $A_{1}$.

No artigo [BBF04], Bekkert, Benkart e Futorny, combinam as técnicas utilizadas em [BB00], [DGO96], e [GP68] para descrever os módulos de peso simples sobre $A_{n}$, com $n \leq \infty$ e $k$ um corpo qualquer. Também foram classificados os tipos de representações em blocos do tipo mansa na categoria de todos os módulos de peso localmente finitos junto com os módulos indecomponíveis de cada um desses blocos. A partir da classificação de certos módulos de peso simples sobre $A_{\infty}$, foram obtidos alguns exemplos de módulos simples $\mathbb{Z}$-graduados para uma álgebra de Heisenberg de dimensão infinita com componentes homogêneas de dimensões infinitas e um central não nulo.

Basearemos essa seção em [BBF04][Seções 2, 3, 4 e 5].

\subsubsection{Módulos de peso sobre $\mathbf{A}=\mathbf{D}(\underline{\sigma}, \underline{\mathbf{a}})$}

Vamos assumir para $1 \leq n \leq \infty$ que $A$ é uma realização da $n$-ésima álgebra de Weyl $A_{n}$ como a álgebra de Weyl generalizada $D(\underline{\sigma}, \underline{a})$, tal que $D=k\left[\tilde{t_{1}}, \ldots, \tilde{t_{n}}\right]$, em que $a_{i}=\tilde{t_{i}}=\partial_{i} x_{i}$ 
$\in D$, cf. Capítulo 1, Seção 1.4. Nesta seção vamos considerar que $k$ é um corpo qualquer. Seja $\mathcal{G}$ o grupo gerado pelos automorfismos $\sigma_{i}(1 \leq i \leq n)$ de $D$, em que $\sigma_{i}\left(\tilde{t}_{j}\right)=\tilde{t}_{j}-\delta_{i j}$. Então $\mathcal{G}$ age no conjunto max $D$ dos ideais maximais de $D$.

Um $A$-módulo $V$ é um módulo de peso se

$$
V=\bigoplus_{\mathfrak{m} \in \mathfrak{m a x} D} V_{\mathfrak{m}} \quad, \quad V_{\mathfrak{m}}=\{v \in V \mid \mathfrak{m} v=\{0\}\}
$$

e se $V_{\mathfrak{m}} \neq\{0\}$, dizemos que $\mathfrak{m}$ é um peso de $V$. O conjunto $\operatorname{supp}(V)=\left\{\mathfrak{m} \in \mathfrak{m a x} D \mid V_{\mathfrak{m}} \neq\right.$ $\{0\}\}$ é o suporte de $V$. Podemos verificar também que $x_{i} V_{\mathfrak{m}} \subseteq V_{\sigma_{i}(\mathfrak{m})}$ e $\partial_{i} V_{\mathfrak{m}} \subseteq V_{\sigma_{i}^{-1}(\mathfrak{m})}$, para $1 \leq i \leq n$ (veja Capítulo 2, Seção 2.5).

Seja $\mathcal{W}(A)$ a categoria dos $A$-módulos de peso. Para cada subconjunto $T \subseteq \max D$ vamos denotar por $\mathcal{W}_{T}(A)$ a subcategoria plena de $\mathcal{W}(A)$ consistindo de todos os módulos de peso $V$ com $\operatorname{supp}(V) \subseteq T$. Todo módulo de peso $V$ pode ser decomposto em uma soma direta de A-submódulos:

$$
V=\bigoplus_{\mathcal{O}} V_{\mathcal{O}} \quad, \quad V_{\mathcal{O}}=\bigoplus_{\mathfrak{m} \in \mathcal{O}} V_{\mathfrak{m}}
$$

em que $\mathcal{O}$ percorre todas as órbitas de $\mathcal{G}$ em max $D$.

Lembremos que se um grupo $G$ age num conjunto $X$ e $x \in X$, o subconjunto de $X$, $o(x)=\{g \cdot x \mid g \in G\}$ é chamado de órbita de $x$.

Então a categoria $\mathcal{W}(A)$ se decompõe em uma soma de subcategorias plenas correspondendo as órbitas de $\mathcal{G}$. Em particular, se $V$ é indecomponível, então seu suporte pertence à uma única órbita.

Uma órbita é cíclica (resp. linear) se $\tau(\mathfrak{m})=\mathfrak{m}$ para algum $\tau \in \mathcal{G}, \tau \neq e(\operatorname{resp} . \tau(\mathfrak{m}) \neq \mathfrak{m}$ para todos $\tau \in \mathcal{G}, \tau \neq e$ ), em que $e$ denota o elemento neutro do grupo $\mathcal{G}$. Se $\operatorname{char}(k)=0$, então toda órbita é linear. Caso contrário, se $\operatorname{char}(k)=p>0$, então toda órbita $\mathcal{O}$ é cíclica e $|\mathcal{O}|<\infty$ se $n<\infty$.

Observação 3.1.1. Seja $V \in \mathcal{W}(A)$ simples e assuma que $\mathfrak{m} \in \operatorname{supp}(V)$. Suponha que a correspondente órbita é linear. Seguindo [BvO97], vamos considerar $A(\mathfrak{m})=A / A \mathfrak{m} \in$ $\mathcal{W}(A)$ e o submódulo maximal $N(\mathfrak{m}) \subset A(\mathfrak{m})$, o qual é a soma de todos os submódulos que interceptam $D / \mathfrak{m}$ trivialmente. Então $V \cong A(\mathfrak{m}) / N(\mathfrak{m})$. Portanto, para órbitas lineares o problema é descrever o submódulo $N(\mathfrak{m})$.

Vamos assumir que $\operatorname{char}(k)=p>0$. A álgebra $A=A_{n}$ é $\mathbb{Z}^{n}$-graduada (veja Capítulo 1, Seção 1.4): $A=\sum_{\underline{j} \in \mathbb{Z}^{n}} A_{\underline{j}}$. Para $\underline{j}=\left(j_{1}, \ldots, j_{n}\right) \in \mathbb{Z}^{n}$, os espaços homogêneos $A_{\underline{j}}=D v_{\underline{j}}$, em que $v_{j}=v_{j_{1}}(1) \cdots v_{j_{n}}(n), v_{j}(i)=x_{i}^{j}, v_{-j}(i)=\partial_{i}^{j}, v_{0}(i)=1$, para todos $i=1, \ldots, n$ e $j>0$. Défine-se a subálgebra de Veronese $A_{[p]}=\sum_{\underline{j \in \mathbb{Z}^{n}}} A_{p \underline{j}}$ (cf. [BvO97]). Se m é um ideal maximal de $D$, então $A_{[p]} \mathfrak{m} \subseteq A_{[p]}$, pois $D \subseteq A_{\underline{0}}$.

Observação 3.1.2. Sejam char $(k)=p>0$ e $V \in \mathcal{W}(A)$ um módulo simples para $A=A_{n}$. Vamos assumir que $V_{\mathfrak{m}} \neq\{0\}$ para algum ideal maximal $\mathfrak{m}$ de $D$. Vamos definir um $A$ módulo por $A(\mathfrak{m})=A \otimes_{A_{[p]}} V_{\mathfrak{m}}$. Então $V \cong A(\mathfrak{m}) / N(\mathfrak{m})$, em que $N(\mathfrak{m})$ é o submódulo maximal em $A(\mathfrak{m})$ que intercepta trivialmente $D / \mathfrak{m}$. O problema de descrever tais módulos simples se reduz a determinar os módulos simples para $A_{[p]} / A_{[p]} \mathfrak{m}$ e o submódulo maximal $N(\mathfrak{m})$.

As Observações 3.1.1 e 3.1 .2 fornecem a construção de todos os $A_{n}$-módulos de peso simples, reduzindo o problema da classificação à descrição do submódulo maximal $N(\mathfrak{m})$. Como a determinação do submódulo maximal $N(\mathfrak{m})$ pode ser extremamente não trivial, 
no que segue vamos adotar uma abordagem alternativa baseada nas ideias de [DGO96] e [BB00].

Dizemos que um ideal maximal $\mathfrak{m}$ de $D$ é um break com respeito a $i$ se $\tilde{t}_{i} \in \mathfrak{m}$ para algum $i \in\{1, \ldots, n\}$. Uma órbita $\mathcal{O}$ é degenerada com respeito a $i$ se ela contém um break com respeito a $i$ (para algum $\mathfrak{m} \in \mathcal{O}$ ). Geralmente por simplicidade dizemos que $\mathcal{O}$ é degenerada sem especificar $i$ ou $\mathfrak{m}$.

Um ideal maximal $\mathfrak{m}$ de $D$ é um break maximal com respeito a $\underline{I}=\underline{I}(\mathfrak{m}) \subseteq\{1, \ldots, n\}$ se $\tilde{t_{i}} \in \mathfrak{m}$ para cada $i \in \underline{I}$, e $\tilde{t_{j}} \notin \tau(\mathfrak{m})$ para todos $j \notin \underline{I}, \tau \in \mathcal{G}$. Definimos $\underline{I}^{c}:=\{1, \ldots, n\} \backslash \underline{I}$ e dizemos que o break maximal tem ordem $|\underline{I}|$ (que pode ser infinita se $n=\infty$ ).

Vejamos que breaks maximais existem para órbitas degeneradas quando $n<\infty$. Suponhamos que $\mathcal{O}=\mathcal{O}(\mathfrak{m})$ é degenerada. Se $\mathfrak{n} \in \mathcal{O}$ é um break com respeito a $i$, então $\tilde{t}_{i} \in \mathfrak{n}$. Além disso, para qualquer produto $\tau=\sigma_{1}^{k_{1}} \cdots \sigma_{n}^{k_{n}} \in \mathcal{G}$ com o termo $\sigma_{i}$ omitido, temos que $\tilde{t_{i}} \in \tau(\mathfrak{n})$, pois $\sigma_{j}\left(\tilde{t}_{i}\right)=\tilde{t}_{i}$ para $j \neq i$. Suponhamos que $\underline{I}=\left\{i_{1}, \ldots, i_{s}\right\} \subseteq\{1, \ldots, n\}$ é um conjunto maximal de índices tal que $\mathcal{O}$ tem breaks $\mathfrak{n}_{i_{1}}, \ldots, \mathfrak{n}_{i_{s}}$ relativos a $i_{1}, \ldots, i_{s}$, respectivamente, e não tem breaks com respeito a qualquer $j \in \underline{I}^{c}$. Vamos assumir $\mathfrak{n}_{i}=\sigma_{1}^{r_{1, i}} \cdots \sigma_{n}^{r_{n, i}}(\mathfrak{m})$ para cada $i \in \underline{I}$. Então para $i \in \underline{I}, \tilde{t}_{i} \in \sigma_{i}^{r_{i, i}}(\mathfrak{m}), \operatorname{logo} \tilde{t_{i}} \in \mathfrak{n}:=\sigma_{i_{1}}^{r_{i_{1}, i_{1}}} \cdots \sigma_{i_{s}}^{r_{i_{s}, i_{s}}}(\mathfrak{m})$, e $\mathfrak{n}$ é um break maximal em $\mathcal{O}$ com respeito a $\underline{I}=\left\{i_{1}, \ldots, i_{s}\right\}$.

Como não é evidente que existem breaks maximais para $A_{\infty}$, vamos assumir que as órbitas degeneradas $\mathcal{O}$, quando estamos considerando $A_{\infty}$, tenham um break maximal $\mathfrak{m}$. Neste caso, o conjunto de breaks $\underline{I}(\mathfrak{m})$ pode ser finito ou infinito.

No que segue, seja $\underline{\tau}(\mathfrak{m})=\left(\tau_{1}, \ldots, \tau_{n}\right)$, em que $\tau_{i}=\sigma_{i}$ se $\sigma_{i}(\mathfrak{m})=\mathfrak{m}$ e $\tau_{i}=e$, caso contrário. Cada $\tau_{i}$ induz um automorfismo em $D / \mathfrak{m}$ (Se $\tau_{i}=e$ é óbvio; se $\tau_{i}=\sigma_{i}$, tal que $\sigma_{i}(\mathfrak{m})=\mathfrak{m}$, basta considerar a projeção canônica $\sigma_{i}: D \rightarrow D / \mathfrak{m}$, temos que $\mathfrak{m} \subseteq k e r\left(\sigma_{i}\right)$, e pela maximalidade de $\mathfrak{m}$, temos que $\sigma_{i}^{\prime}: D / \mathfrak{m} \rightarrow D / \mathfrak{m}$ é um isomorfismo).

\subsubsection{Categoria $\mathcal{C}_{\mathcal{O}}$ e seu esqueleto}

Começaremos com os conceitos abstratos necessários para descrever os módulos sobre a álgebra de Weyl, que podem ser encontrados em [GR97][Cap. 2]. Ultimamente surgiram muitas categorias particulares determinadas por esses módulos.

\section{Categorias}

Seja $\mathbb{F}$ um corpo. Uma categoria $\mathcal{C}$ é dita uma $\mathbb{F}$-categoria se cada conjunto de morfismos $\mathcal{C}(\alpha, \beta)$ é equipado com uma estrutura de $\mathbb{F}$-bimódulo, a composição é $\mathbb{F}$-linear com respeito as estruturas de $\mathbb{F}$-módulo à esquerda e à direita, e $(\alpha \lambda) \beta=\alpha(\lambda \beta)$ para quaisquer morfismos possíveis $\alpha, \beta(\lambda \in \mathbb{F})$. Se adicionalmente, $\alpha \lambda=\lambda \alpha$ para todos morfismos $\alpha(\lambda \in \mathbb{F})$, então dizemos que $\mathcal{C}$ é uma categoria $\mathbb{F}$-linear. Uma $\mathbb{F}$-álgebra $A$ dá origem à uma $\mathbb{F}$-categoria com somente um objeto $\alpha$ tal que $\mathcal{C}(\alpha, \alpha)=A$, e a composição é a multiplicação em $A$.

Para qualquer categoria $\mathcal{C}$, podemos associar uma categoria $\mathbb{F}$-linear $\mathbb{F} \mathcal{C}$ cujos objetos são os mesmos $O b(\mathcal{C})=O b(\mathbb{F} \mathcal{C})$, e o espaço de morfismos $\mathbb{F} \mathcal{C}(\alpha, \beta)$ tem como base sobre $\mathbb{F}$ os elementos de $\mathcal{C}(\alpha, \beta)$. A composição em $\mathbb{F} \mathcal{C}$ é a extensão $\mathbb{F}$-linear da composição em $\mathcal{C}$.

Um funtor $\mathbb{F}$-linear entre duas categorias $\mathbb{F}$-lineares $\mathcal{C}$ e $\mathcal{D}$ é um funtor $F: \mathcal{C} \rightarrow \mathcal{D}$ cujas aplicações $F(\alpha, \beta): \mathcal{C}(\alpha, \beta) \rightarrow \mathcal{D}(F \alpha, F \beta)$ são $\mathbb{F}$-lineares para todos $\alpha, \beta \in O b(\mathcal{C})$.

Sejam $\mathbb{F}$-mod a categoria dos $\mathbb{F}$-espaços vetoriais e AbGroups dos grupos abelianos. Dada uma $\mathbb{F}$-categoria $\mathcal{C}$, vamos denotar por $\mathcal{C}$-mod a categoria de todos os funtores aditivos $M: \mathcal{C} \rightarrow$ AbGroups (observamos que o conjunto de todos os funtores de uma $\mathcal{C}$ em uma categoria $\mathcal{D}$ é uma categoria, porque podemos definir um morfismo entre dois funtores do conjunto). Os funtores $M$ são chamados de $\mathcal{C}$-módulos, ou mais precisamente, $\mathcal{C}$-módulos à 
esquerda. Para $\alpha \in O b(\mathcal{C})$, os elementos de $M(\alpha)$ são os elementos do módulo $M$ (em $\alpha$ ). Fazendo a ação do modo que estamos acostumados, escrevemos au ao invés de $M(a) u$ para $u \in M(\alpha)$ e $a \in \mathcal{C}(\alpha, \beta)$. Para cada $\alpha \in O b(\mathcal{C})$, o grupo $M(\alpha)$ torna-se um $\mathbb{F}$-espaço vetorial se tomarmos $\lambda u=\left(\lambda 1_{\alpha}\right) u$, para todos $u \in M(\alpha)$ e $\lambda \in \mathbb{F}$. Por $\mathcal{C}$-fdmod, indicamos a subcategoria plena de todos os objetos $M$ em $\mathcal{C}$-mod que possuem dimensão finita localmente, isto é, $\operatorname{dim}_{\mathbb{F}}(M(\alpha))<\infty$, para todo $\alpha \in O b(\mathcal{C})$.

Agora vamos definir alguns conceitos que já são conhecidos na teoria dos módulos, mas que considerando o conceito de módulo dado acima, tem suas particularidades. São eles: submódulo, módulo simples, soma direta e módulo indecomponível.

Se $N$ é um $\mathcal{C}$-submódulo de $M$, então $N(\alpha)$ é um subespaço de $M(\alpha)$ para todo $\alpha \in$ $O b(\mathcal{C})$, e $a u \in N(\beta)$ para todos $a \in \mathcal{C}(\alpha, \beta)$ e $u \in N(\alpha)$. O módulo $M$ é simples se ele não tem $\mathcal{C}$-submódulos não triviais; enquanto $M$ é uma soma direta $M=M_{1} \oplus M_{2}$ se $M_{1}$ e $M_{2}$ são submódulos tais que $M(\alpha)=M_{1}(\alpha) \oplus M_{2}(\alpha)$ para todo $\alpha \in O b(\mathcal{C})$. O módulo $M$ é indecomponivel se ele não pode ser escrito como uma soma direta $M=M_{1} \oplus M_{2}$ de submódulos próprios.

$\mathcal{C}$-módulos à direita podem ser definidos como $\mathcal{C}^{o p}$-módulos, em que $\mathcal{C}^{o p}$ é a categoria oposta à $\mathcal{C}$. Neste caso escrevemos va para $v \in M(\beta)$ e para $a \in \mathcal{C}(\alpha, \beta)=\mathcal{C}^{o p}(\beta, \alpha)$. A categoria dos $\mathcal{C}$-módulos à direita será denotada por mod-C e a subcategoria plena dos objetos que possuem dimensão finita localmente por fdmod-C .

Para $\mathbb{F}$-categorias $\mathcal{C}$ e $\mathcal{D}$ um $\mathcal{C}$-D -bimódulo é um funtor aditivo $B: \mathcal{C} \times \mathcal{D}^{o p} \rightarrow$ AbGroups. Se $u \in B(\gamma, \delta)$, então $u$ é um elemento do módulo $B$ com origem $\gamma$ e destino $\delta$, e escrevemos $a u b$ ao invés de $B(a, b) u$ para $a \in \mathcal{C}\left(\gamma, \gamma^{\prime}\right)$ e $b \in \mathcal{D}\left(\delta^{\prime}, \delta\right)$. Qualquer $\mathbb{F}$-categoria $\mathcal{C}$ pode ser vista como um $\mathcal{C}$-C -bimódulo aplicando o par de objetos $(\beta, \gamma)$ em $\mathcal{C}(\beta, \gamma) \in O b$ (AbGroups).

Dadas duas $\mathbb{F}$-categorias $\mathcal{C}$ e $\mathcal{D}$, a categoria $\mathcal{E}$ é o produto tensorial $\mathcal{E}=\mathcal{C} \otimes_{\mathbb{F}} \mathcal{D}$ das categorias $\mathcal{C}$ e $\mathcal{D}$ se $O b(\mathcal{E})=O b(\mathcal{C}) \times O b(\mathcal{D}), \mathcal{E}((\alpha, \beta),(\gamma, \delta))=\mathcal{C}(\alpha, \gamma) \otimes_{\mathbb{F}} \mathcal{D}(\beta, \delta)$, e a composição de morfismos é definida da seguinte maneira $(a \otimes b)(c \otimes d)=a c \otimes b d$.

A categoria $\mathcal{C}$ é dita básica se:

- Todos os seus objetos são não isomorfos, tomados dois a dois;

- Para cada objeto $\alpha$ não existem idempotentes não triviais em $\mathcal{C}(\alpha, \alpha)$.

Uma subcategoria plena $\mathcal{S}$ é um esqueleto de uma categoria $\mathcal{C}$ se ela é básica, e cada objeto $\alpha \in O b(\mathcal{C})$ é isomorfo à um somando direto de uma soma direta (finita) de alguns objetos de $\mathcal{S}$. Se a categoria $\mathcal{C}$ tem a propriedade de ter uma única decomposição em soma direta, então ela tem um esqueleto que é único a menos de isomorfismo. O funtor inclusão natural $\mathcal{I}: \mathcal{S} \rightarrow \mathcal{C}$ de um esqueleto $\mathcal{S}$ em $\mathcal{C}$ é uma equivalência de categorias. Por conta desse funtor, $\mathcal{C}$ é um $\mathcal{C}$-S $\mathcal{S}$-bimódulo de maneira óbvia.

\section{Quivers}

Um quiver $Q$ (ou aljava) é uma upla $\left(Q_{0}, Q_{1}, \mathfrak{s}, \mathfrak{e}\right)$ consistindo de um conjunto $Q_{0}$ de vértices, um conjunto $Q_{1}$ de flechas, e aplicações $\mathfrak{s}, \mathfrak{e}: Q_{1} \rightarrow Q_{0}$ que especificam os vértices iniciais e finais. Um caminho $p$ em $Q$ de comprimento $l(p)=n \geq 1$ é uma sequência de flechas $a_{n}, \ldots, a_{1}$ tais que $\mathfrak{s}\left(a_{i+1}\right)=\mathfrak{e}\left(a_{i}\right)$ para $1 \leq i<n, \mathfrak{s}\left(a_{1}\right)=\mathfrak{s}(p)$ (início/origem de $p$ ) e $\mathfrak{e}\left(a_{n}\right)=\mathfrak{e}(p)$ (término/destino de $p$ ). Então a concatenação $p^{\prime} p$ de dois caminhos $p, p^{\prime}$ é definida de maneira natural desde que $\mathfrak{s}\left(p^{\prime}\right)=\mathfrak{e}(p)$. Cada vértice $a \in Q_{0}$ determina um caminho $1_{a}$ (de comprimento 0 ) conhecido como caminho trivial em que $\mathfrak{s}\left(1_{a}\right)=\mathfrak{e}\left(1_{a}\right)=a$. Um caminho de comprimento $l \geq 1$ é chamado de ciclo se $\mathfrak{s}\left(a_{1}\right)=\mathfrak{e}\left(a_{l}\right)$, ou seja, se começa e termina no mesmo vértice. Um ciclo de comprimento 1 é chamado de loop (ou laço). 
Se $Q=\left(Q_{0}, Q_{1}, \mathfrak{s}, \mathfrak{e}\right)$ é um quiver, diremos que $Q^{\prime}=\left(Q_{0}^{\prime}, Q_{1}^{\prime}, \mathfrak{s}^{\prime}, \mathfrak{e}^{\prime}\right)$ é um subquiver (ou subaljava) de $Q$ se $Q_{0}^{\prime} \subseteq Q_{0}, Q_{1}^{\prime} \subseteq Q_{1}$ e a restrições de $\mathfrak{s}$ e $\mathfrak{t}$ a $Q_{1}^{\prime}$ são iguais a $\mathfrak{s}^{\prime}$ e $\mathfrak{e}^{\prime}$, respectivamente.

Um quiver $Q$ determina uma categoria $\mathfrak{p a t h} Q$ em que os objetos são os vértices de $Q$ e os morfismos de um vértice $a$ para um vértice $b$ são os caminhos de $a$ para $b$. A composição em $\mathfrak{p a t h} Q$ dos caminhos de comprimento positivo é simplesmente a concatenação, e o caminho $1_{a}$ age como identidade em todos os caminhos para os quais a composição faça sentido.

A correspondente álgebra de caminhos, que abreviaremos simplesmente por $\mathbb{F} Q$, tem uma $\mathbb{F}$-base consistindo dos caminhos de $Q$ com multiplicação dada pela concatenação dos caminhos. Se $\mathcal{R}$ é o ideal de $\mathbb{F} Q$ gerado por uma família $\left\{\rho_{i}\right\}$ de morfismos, dizemos que $\mathbb{F} Q / \mathcal{R}$ é uma categoria $\mathbb{F}$-linear definida pelo quiver $Q$ e por relações $\left\{\rho_{i}\right\}$.

Exemplo 3.1.1. Seja $Q$ o quiver

$$
a^{\circ} \beta
$$

consistindo de um simples ponto e um loop simples. A base da álgebra de caminhos $\mathbb{F} Q$ é $\left\{1_{a}, \beta, \beta^{2}, \ldots, \beta^{l}, \ldots\right\}$ e a multiplicação dos vetores da base é dada por:

$$
\begin{gathered}
1_{a} \beta^{l}=\beta^{l} 1_{a}=\beta^{l} \quad, \text { para todo } l \geq 0 e \\
\beta^{l} \beta^{r}=\beta^{l+r} \quad, \text { para todos } l, r \geq 0
\end{gathered}
$$

em que $\beta^{0}=1_{a}$. Então $\mathbb{F} Q$ é isomorfa à álgebra de polinômios $\mathbb{F}[t]$ com uma variável; o isomorfismo é induzido pela aplicação $\mathbb{F}$-linear $1_{a} \mapsto 1$ e $\beta \mapsto t$.

\section{Categoria $\mathcal{C}_{\mathcal{O}}$}

Vamos mostrar que a categoria $\mathcal{W}_{\mathcal{O}}(A)$ dos módulos de peso sobre a álgebra de Weyl $A=A_{n}(1 \leq n \leq \infty)$ com suporte na órbita $\mathcal{O}$ (no caso $n=\infty$ assumimos que se $\mathcal{O}$ é degenerada ela possui um break maximal), é equivalente à $\mathcal{C}_{\mathcal{O}^{-}}$mod, a categoria dos módulos sobre uma certa categoria $\mathcal{C}_{\mathcal{O}}$ primeiramente introduzida em [DGO96].

Vamos assumir que $\mathfrak{m}$ é um break maximal fixado na órbita $\mathcal{O}$ se $\mathcal{O}$ é degenerada, e é qualquer elemento fixado de $\mathcal{O}$ caso contrário. Para um dado $\mathfrak{n} \in \mathcal{O}$ denotaremos por $\sigma_{\mathfrak{n}} \mathrm{O}$ elemento de $\mathcal{G} / \mathfrak{s t a b}(\mathfrak{m})$ tal que $\mathfrak{n}=\sigma_{\mathfrak{n}}(\mathfrak{m})$ (em que $\mathfrak{s t a b}(\mathfrak{m})$ é o subgrupo estabilizador de $\mathfrak{m}$ em $\mathcal{G}$; lembremos que se um grupo $G$ age em um conjunto $X$ e $x \in X$, o subgrupo de $G$, $G_{x}=\{g \in G \mid g x=x\}$ é chamado de estalizador de $\left.x\right)$. Então, $\sigma_{\mathfrak{n}}$ induz um isomorfismo $D / \mathfrak{m} \rightarrow D / \mathfrak{n}$, que também denotaremos por $\sigma_{\mathfrak{n}}$ (seja $\sigma_{\mathfrak{n}}: D \rightarrow D / \mathfrak{n}$ o homomorfismo sobrejetor canônico; como $\operatorname{ker}\left(\sigma_{\mathfrak{n}}\right)=\mathfrak{n}$ temos que $\mathfrak{m} \subseteq \operatorname{ker}\left(\sigma_{\mathfrak{n}}\right)$; mas $\mathfrak{m}$ é um ideal maximal, $\operatorname{logo} \operatorname{ker}\left(\sigma_{\mathfrak{n}}\right)=\mathfrak{m}$; portanto $\left.D / \mathfrak{m} \cong D / \mathfrak{n}\right)$ e seu inverso induz um isomorfismo $\sigma_{\mathfrak{n}}^{-1}: D / \mathfrak{n} \rightarrow$ $D / \mathfrak{m}$.

Definimos $\mathcal{C}_{\mathcal{O}}$ como a $D / \mathfrak{m}$-categoria com $\operatorname{Ob}\left(\mathcal{C}_{\mathcal{O}}\right)=\mathcal{O}$, gerados sobre $D / \mathfrak{m}$ pelo conjunto de morfismos $\left\{X_{\mathfrak{n}, i}, Y_{\mathfrak{n}, i} \mid \mathfrak{n} \in \mathcal{O}, 1 \leq i \leq n\right\}$, em que $X_{\mathfrak{n}, i}: \mathfrak{n} \rightarrow \sigma_{i}(\mathfrak{n})$ e $Y_{\mathfrak{n}, i}: \sigma_{i}(\mathfrak{n}) \rightarrow \mathfrak{n}$, sujeitos às relações:

- $X_{\mathfrak{n}, i} \lambda=\lambda X_{\mathfrak{n}, i} \quad, \quad Y_{\mathfrak{n}, i} \lambda=\lambda Y_{\mathfrak{n}, i} \quad, \quad Y_{\mathfrak{n}, i} X_{\mathfrak{n}, i}=\sigma_{\mathfrak{n}}^{-1}\left(\bar{t}_{i}\right) 1_{\mathfrak{n}} \quad$ e $\quad X_{\mathfrak{n}, i} Y_{\mathfrak{n}, i}=\sigma_{\mathfrak{n}}^{-1}\left(\bar{t}_{i}\right) 1_{\sigma_{i}(\mathfrak{n})}$, $\bar{t}_{i}=\tilde{t}_{i}+\mathfrak{n}$, para todos $\lambda \in D / \mathfrak{m}, \mathfrak{n} \in \mathcal{O}$, e todo $1 \leq i \leq n$ tal que $\sigma_{i}(\mathfrak{m}) \neq \mathfrak{m}$

- $X_{\mathfrak{n}, i} \lambda=\sigma_{i}(\lambda) X_{\mathfrak{n}, i} \quad, \quad Y_{\mathfrak{n}, i} \sigma_{i}(\lambda)=\lambda Y_{\mathfrak{n}, i} \quad, \quad Y_{\mathfrak{n}, i} X_{\mathfrak{n}, i}=\sigma_{\mathfrak{n}}^{-1}\left(\bar{t}_{i}\right) 1_{\mathfrak{n}} \quad$ e $\quad X_{\mathfrak{n}, i} Y_{\mathfrak{n}, i}=$ $\sigma_{i} \sigma_{\mathfrak{n}}^{-1}\left(\bar{t}_{i}\right) 1_{\sigma_{i}(\mathfrak{n})}, \bar{t}_{i}=\tilde{t}_{i}+\mathfrak{n}$, para todos $\lambda \in D / \mathfrak{m}, \mathfrak{n} \in \mathcal{O}$, e todo $1 \leq i \leq n$ tal que $\sigma_{i}(\mathfrak{m})=\mathfrak{m}$;

- $U_{\mathfrak{n}, i} V_{\mathfrak{p}, j}-V_{\mathfrak{q}, j} U_{\mathfrak{r}, i}=0$ para todo $j \neq i$ e para todos possíveis $U, V \in\{X, Y\}, \mathfrak{n}, \mathfrak{p}, \mathfrak{q}, \mathfrak{r}$ $\in \mathcal{O}$, para os quais a última igualdade faça sentido. 
Observamos que a categoria $\mathcal{C}_{\mathcal{O}}$ é $\mathbb{F}$-linear quando $\sigma_{i}(\mathfrak{m}) \neq \mathfrak{m}$ para todo $i$; em particular, $\mathcal{C}_{\mathcal{O}}$ é sempre $\mathbb{F}$-linear quando $\mathbb{F}$ tem característica 0 (pois nesse caso a órbita é linear, ou seja, $\tau(\mathfrak{m}) \neq \mathfrak{m}$, para todo $\tau \neq e \in \mathcal{G})$.

Proposição 3.1.1. Seja $A=A_{n}$, a n-ésima álgebra de Weyl sobre o corpo $k$, e seja $\mathcal{O}$ uma órbita de max $D$ (se $n=\infty$ e $\mathcal{O}$ é degenerada vamos assumir que ela tem um break maximal). Então $\mathcal{W}_{\mathcal{O}}(A) \approx \mathcal{C}_{\mathcal{O}^{-} \text {mod. }}$

Demonstração. Vamos assumir que $\mathfrak{m}$ é o ideal maximal designado de $\mathcal{O}$ (o ideal maximal $\mathfrak{m}$ utilizado na definição da categoria $\left.\mathcal{C}_{\mathcal{O}}\right)$. Seja $V=\bigoplus_{\mathfrak{n} \in \mathcal{O}} V_{\mathfrak{n}}$ pertencente à $\mathcal{W}_{\mathcal{O}}(A)$. Para cada $\mathfrak{n} \in \mathcal{O}$ seja $M_{V}(\mathfrak{n}):=V_{\mathfrak{n}}$.

Como $V_{\mathfrak{n}}$ é $D$-módulo e $\mathfrak{n} V_{\mathfrak{n}}=\{0\}$, temos que $V_{\mathfrak{n}}$ é $D / \mathfrak{n}$-espaço vetorial. Usando o isomorfismo $\sigma_{\mathfrak{n}}: D / \mathfrak{m} \rightarrow D / \mathfrak{n}$, podemos tomar $M_{V}(\mathfrak{n})$ como $D / \mathfrak{m}$-espaço vetorial via $\bar{d} v:=$ $\sigma_{\mathfrak{n}}(\bar{d}) v,(\bar{d}=d+\mathfrak{m})$. Para $v \in M_{V}(\mathfrak{n})$ e $w \in M_{V}\left(\sigma_{i}(\mathfrak{n})\right)$, definimos $X_{\mathfrak{n}, i} v:=x_{i} v \in M_{V}\left(\sigma_{i}(\mathfrak{n})\right)$ (pois $\left.x_{i} V_{\mathfrak{n}} \subseteq V_{\sigma_{i}(\mathfrak{n})}\right)$ e $Y_{\mathfrak{n}, i} w:=\partial_{i} w \in M_{V}(\mathfrak{n})$ (pois $\left.\partial_{i} V_{\sigma_{i}(\mathfrak{n})} \subseteq V_{\sigma_{i}^{-1}\left(\sigma_{i}(\mathfrak{n})\right)}=V_{\mathfrak{n}}\right)$. Então se $\bar{d} \in$ $D / \mathfrak{m}$, temos

$$
\begin{aligned}
X_{\mathfrak{n}, i} \bar{d} v & =x_{i} \sigma_{\mathfrak{n}}(\bar{d}) v=\sigma_{i}\left(\sigma_{\mathfrak{n}}(\bar{d})\right) x_{i} v \\
& =\left\{\begin{array}{lll}
\bar{d} X_{\mathfrak{n}, i} v & \text { se } & \sigma_{i}(\mathfrak{m}) \neq \mathfrak{m} \\
\sigma_{i}(\bar{d}) X_{\mathfrak{n}, i} v & \text { se } & \sigma_{i}(\mathfrak{m})=\mathfrak{m}
\end{array}\right.
\end{aligned}
$$

visto que $\sigma_{i}(\mathfrak{n})=\mathfrak{n}$ para todo $\mathfrak{n} \in \mathcal{O}$, e $\sigma_{i}\left(\sigma_{\mathfrak{n}}(\bar{d})\right)=\sigma_{\mathfrak{n}}\left(\sigma_{i}(\bar{d})\right)$, sempre que $\sigma_{i}(\mathfrak{m})=\mathfrak{m}$. Da mesma forma $Y_{\mathfrak{n}, i} \bar{d} w=\bar{d} Y_{\mathfrak{n}, i} w$. Além disso, $Y_{\mathfrak{n}, i} X_{\mathfrak{n}, i} v=\partial_{i} x_{i} v=\tilde{t}_{i} v=(\tilde{t} i+\mathfrak{n}) v=\sigma_{\mathfrak{n}}^{-1}\left(\bar{t}_{i}\right) v$ para $\bar{t}_{i}=\tilde{t}_{i}+\mathfrak{n}$, e $X_{\mathfrak{n}, i} Y_{\mathfrak{n}, i} w=x_{i} \partial_{i} w=\sigma_{i}\left(\tilde{t}_{i}\right) w=\left(\sigma_{i}\left(\tilde{t}_{i}\right)+\sigma_{i}(\mathfrak{n})\right) w=\sigma_{\mathfrak{n}}^{-1}\left(\bar{t}_{i}\right) w$. A razão para esta última igualdade é que existe uma ação de $\mathcal{G}$ em $\mathcal{G} / \mathfrak{s t a b}(\mathfrak{m})$, e sob estão ação $\sigma_{i} \sigma_{\mathfrak{n}}=\sigma_{\sigma_{i}(\mathfrak{n})}$. Portanto, $M_{V}\left(M_{V}: \mathcal{C}_{\mathcal{O}} \rightarrow D / \mathfrak{m}\right.$-mod $)$ é um $\mathcal{C}_{\mathcal{O}}$-módulo, e temos um funtor

$$
F: \mathcal{W}_{\mathcal{O}}(A) \rightarrow \mathcal{C}_{\mathcal{O}^{-} \bmod } \quad, \quad V \mapsto M_{V}
$$

Reciprocamente, para cada $M \in \mathcal{C}_{\mathcal{O}^{-}}$mod, vamos definir o $A$-módulo $V_{M}:=\bigoplus_{\mathfrak{n} \in \mathcal{O}} M(\mathfrak{n})$, em que $d v:=\sigma_{\mathfrak{n}}^{-1}(\bar{d}) v, \bar{d}=d+\mathfrak{n} \in D / \mathfrak{n}, x_{i} v:=X_{\mathfrak{n}, i} v$, e $\partial_{i} v:=Y_{\sigma_{i}^{-1}(\mathfrak{n}), i} v$, para $v \in M(\mathfrak{n})$. Isto resulta em um funtor

$$
F^{\prime}: \mathcal{C}_{\mathcal{O}^{-} \bmod } \rightarrow \mathcal{W}_{\mathcal{O}}(A) \quad, \quad M \mapsto V_{M}
$$

que é o inverso de $F$. Portanto as duas categorias são equivalentes.

\section{Esqueleto de $\mathcal{C}_{\mathcal{O}}$}

Para uma dada órbita $\mathcal{O}$ vamos definir o conjunto $\mathfrak{B}_{\mathcal{O}}$ de acordo com as regras descritas abaixo. Se $\mathcal{O}$ é não degenerada, então $\mathfrak{B}_{\mathcal{O}}:=\{\mathfrak{m}\}$, para $\mathfrak{m} \in \mathcal{O}$ fixado, usado para definir a categoria $\mathcal{C}_{\mathcal{O}}$, e $\underline{I}(\mathfrak{m})=\emptyset$. Se $\mathcal{O}$ é uma órbita degenerada linear, então $\mathfrak{m} \in \mathcal{O}$ é o break maximal fixado usado na construção de $\mathcal{C}_{\mathcal{O}}$; vamos assumir que o conjunto de breaks é $\underline{I}=\underline{I}(\mathfrak{m})=\left\{i_{1}, \ldots, i_{s}\right\} ;$ seja $\mathfrak{B}_{\mathcal{O}}:=\left\{\sigma_{i_{1}}^{\delta_{1}} \cdots \sigma_{i_{s}}^{\delta_{s}}(\mathfrak{m}) \mid \delta_{j} \in\{0,1\}\right\}$ (em que somente uma quantidade finita dos $\delta_{j}$ é não nula, quando $\left.n=\infty\right)$; neste caso, para cada ideal maximal $\mathfrak{n}=\sigma_{i_{1}}^{\delta_{1}} \cdots \sigma_{i_{s}}^{\delta_{s}}(\mathfrak{m}) \in \mathfrak{B}_{\mathcal{O}}$, seja

$$
\mathcal{O}_{\mathfrak{n}}:=\left\{\sigma_{1}^{\gamma_{1}} \cdots \sigma_{n}^{\gamma_{n}}(\mathfrak{n}) \mid \gamma_{i}=(-1)^{\delta_{i}+1} k, k \in \mathbb{N} \text { se } i \in \underline{I}, \text { e } \gamma_{i} \in \mathbb{Z} \text { se } i \in \underline{I}^{c}\right\},
$$

(em que somente uma quantidade finita $\operatorname{dos} \gamma_{i}$ é não nula, quando $n=\infty$ ). Se $\mathcal{O}$ é uma órbita degenerada cíclica, e $\mathfrak{m} \in \mathcal{O}$ é o break maximal usado para definir $\mathcal{C}_{\mathcal{O}}$, então $\mathfrak{B}_{\mathcal{O}}:=\{\mathfrak{m}\}$ e 
$\mathcal{O}_{\mathfrak{m}}:=\mathcal{O}$.

Como $\sigma_{i}\left(\tilde{t}_{j}\right)=\tilde{t_{j}}$, quando $i \neq j$, existe a seguinte relação de equivalência: $\mathfrak{n} \sim \sigma_{i}(\mathfrak{n})$ se, e somente se, $\tilde{t}_{i} \notin \mathfrak{n}$. Vamos introduzir uma relação de equivalência $\sim$ no conjunto dos ideais maximais $\mathfrak{n} \in \mathfrak{m a x} D$. Esta relação é uma extensão transitiva da especificada acima. Vejamos:

Lema 3.1.1. Suponhamos que $\mathfrak{n}$ e p pertencem a $\mathcal{O}$. Então $\mathfrak{n} \sim \mathfrak{p}$ se, e somente se, $\mathfrak{n}$ e $\mathfrak{p}$ são isomorfos em $\mathcal{C}_{\mathcal{O}}$.

Demonstração. Considerando a construção da categoria $\mathcal{C}_{\mathcal{O}}$, seja $\mathfrak{m}$ o break maximal fixado de $\mathcal{O}$ se $\mathcal{O}$ é degenerada e o único elemento de $\mathfrak{B}_{\mathcal{O}}$ se $\mathcal{O}$ é não degenerada. Vamos assumir para cada $\mathfrak{n} \in \mathcal{O}, \sigma_{\mathfrak{n}} \in \mathcal{G} / \mathfrak{s t a} \mathfrak{b}(\mathfrak{m})$ satisfazendo $\sigma_{\mathfrak{n}}(\mathfrak{m})=\mathfrak{n}$ como na demonstração da Proposição 3.1.1.

É suficiente argumentar para qualquer $\mathfrak{n} \in \mathcal{O}$ que, $\mathfrak{n} \sim \sigma_{i}(\mathfrak{n})$ se, e somente se, $\mathfrak{n}$ e $\sigma_{i}(\mathfrak{n})$ são isomorfos em $\mathcal{C}_{\mathcal{O}}$.

Vamos assumir que $\mathfrak{n} \sim \sigma_{i}(\mathfrak{n})$. Então $\tilde{t_{i}} \notin \mathfrak{n}, \operatorname{logo}\left(\tilde{t}_{i}+\mathfrak{n}\right) \neq 0$. Como $\sigma_{\mathfrak{n}}^{-1}: D / \mathfrak{n} \rightarrow D / \mathfrak{m}$ é um isomorfismo, $\sigma_{\mathfrak{n}}^{-1}\left(\tilde{t}_{i}+\mathfrak{n}\right) \neq 0$. Pela definição de $\mathcal{C}_{\mathcal{O}}, X_{\mathfrak{n}, i}$ e $Y_{\mathfrak{n}, i}$ são invertíveis em $\mathcal{C}_{\mathcal{O}}$. Portanto $\mathfrak{n}$ e $\sigma_{i}(\mathfrak{n})$ são isomorfos em $\mathcal{C}_{\mathcal{O}}$.

Reciprocamente, suponhamos que $\mathfrak{n}$ e $\sigma_{i}(\mathfrak{n})$ são isomorfos em $\mathcal{C}_{\mathcal{O}}$. Segue da definição dos morfismos em $\mathcal{C}_{\mathcal{O}}$ que $\mathcal{C}_{\mathcal{O}}\left(\mathfrak{n}, \sigma_{i}(\mathfrak{n})\right)=(D / \mathfrak{m}) X_{\mathfrak{n}, i}$ e $\mathcal{C}_{\mathcal{O}}\left(\sigma_{i}(\mathfrak{n}), \mathfrak{n}\right)=(D / \mathfrak{m}) Y_{\mathfrak{n}, i}$, para $1 \leq i \leq n$. Então $X_{\mathfrak{n}, i}$ e $Y_{\mathfrak{n}, i}$ são isomorfismos. Se $\tilde{t}_{i} \in \mathfrak{n}$, então $\sigma_{\mathfrak{n}}^{-1}\left(\tilde{t}_{i}+\mathfrak{n}\right)=0 \in D / \mathfrak{m}$ e $Y_{\mathfrak{n}, i} X_{\mathfrak{n}, i}=$ $\sigma_{\mathfrak{n}}^{-1}\left(\tilde{t}_{i}+\mathfrak{n}\right) 1_{\mathfrak{n}}=0$, uma contradição. Logo $\tilde{t}_{i} \notin \mathfrak{n}$ e, portanto, $\mathfrak{n} \sim \sigma_{i}(\mathfrak{n})$.

Observação 3.1.3. Seja $\mathfrak{n}=\sigma_{i_{1}}^{\delta_{1}} \cdots \sigma_{i_{s}}^{\delta_{s}}(\mathfrak{m}) \in \mathfrak{B}_{\mathcal{O}}$. Se $j \in \underline{I}$ e $\delta_{j}=1$, então $\tilde{t_{j}} \notin \mathfrak{n}$, logo $\mathfrak{n} \sim \sigma_{j}(\mathfrak{n})$. Se $j \in \underline{I}^{c}$, ent $\tilde{a} o \tilde{t_{j}} \notin \mathfrak{m}$, assim $\tilde{t_{j}} \notin \mathfrak{n}$ e $\mathfrak{n} \sim \sigma_{j}(\mathfrak{n})$. Portanto, para $\mathfrak{n} \in \mathfrak{B}_{\mathcal{O}}, a$ classe de equivalência de $\mathfrak{n}$ é exatamente $\mathcal{O}_{\mathfrak{n}}$, e $\mathcal{O}$ é a união disjunta dos conjuntos $\mathcal{O}_{\mathfrak{n}}, \mathfrak{n} \in$ $\mathfrak{B}_{\mathcal{O}}$.

Corolário 3.1.1. A subcategoria plena $\mathcal{S}_{\mathcal{O}} \operatorname{com} O b\left(\mathcal{S}_{\mathcal{O}}\right)=\mathfrak{B}_{\mathcal{O}}$ é um esqueleto da categoria $\mathcal{C}_{\mathcal{O}}$

Demonstração. Vamos assumir que $\mathfrak{m}$ é o ideal maximal designado em $\mathcal{O}$, utilizado para definir a categoria $\mathcal{C}_{\mathcal{O}}$, e o correspondente conjunto de breaks é $\underline{I}=\underline{I}(\mathfrak{m})=\left\{i_{1}, \ldots, i_{s}\right\}$ (que é vazio se $\mathcal{O}$ é não degenerada). No caso degenerado, o ideal maximal $\sigma_{i_{1}}^{\delta_{1}} \cdots \sigma_{i_{s}}^{\delta_{s}}(\mathfrak{m})$ tem break em $\left\{i_{k} \mid \delta_{k}=0\right\}$, pois se $\delta_{k}=0$ em $\sigma_{i_{1}}^{\delta_{1}} \cdots \sigma_{i_{s}}^{\delta_{s}}$ então $\tilde{i_{k}}=\sigma_{i_{1}}^{\delta_{1}} \cdots \tilde{\sigma_{i_{s}}}\left(\tilde{t_{i_{k}}}\right) \in$ $\sigma_{i_{1}}^{\delta_{1}} \cdots \sigma_{i_{s}}^{\delta_{s}}(\mathfrak{m})$, visto que $\tilde{i_{k}} \in \mathfrak{m}$. Assim, esses ideais são dois a dois não isomorfos. De fato, sejam $\mathfrak{n}_{i_{k}}$ e $\mathfrak{n}$ dois ideais maximais da forma $\sigma_{i_{1}}^{\delta_{1}} \cdots \sigma_{i_{s}}^{\delta_{s}}(\mathfrak{m})$, tais que $\mathfrak{n}_{i_{k}}$ tem break em $i_{k}$ e $\mathfrak{n}$ não possui break em $i_{k}$. Então, $\tilde{t_{i_{k}}} \in \mathfrak{n}_{i_{k}}$ se, e somente se, $\sigma_{i_{k}}\left(\mathfrak{n}_{i_{k}}\right)$ não é equivalente a $\mathfrak{n}_{i_{k}}$ se, e somente se, $\sigma_{i_{k}}\left(\mathfrak{n}_{i_{k}}\right) \not \mathfrak{n}_{i_{k}}$. Por outro lado, $\tilde{t_{i_{k}}} \notin \mathfrak{n}$ e $\tilde{t_{i_{k}}} \notin \sigma_{i_{k}}\left(\mathfrak{n}_{i_{k}}\right)$, isto é, $\sigma_{i_{k}}\left(\mathfrak{n}_{i_{k}}\right) \cong \mathfrak{n}$. Logo, $\mathfrak{n} ¥ \mathfrak{n}_{i_{k}}$ (observamos que esse isomorfismo em $\mathcal{C}_{\mathcal{O}}$ é uma relação de equivalência pelo Lema 3.1.1, logo é transitivo).

Além disso, se $\mathfrak{n}=\sigma_{1}^{r_{1}} \cdots \sigma_{n}^{r_{n}}(\mathfrak{m}) \in \mathcal{O}$ (com somente uma quantidade finita de $r_{i}$ 's não nula, quando $n=\infty$ ), então pelo Lema 3.1.1, $\mathfrak{n}$ é isomorfo a $\sigma_{i_{1}}^{\delta_{1}} \cdots \sigma_{i_{s}}^{\delta_{s}}(\mathfrak{m}) \in \mathfrak{B}_{\mathcal{O}}$ tal que $\delta_{i}=1$ sempre que $i \in \underline{I}$ e $r_{i} \geq 1$ (pois $\tilde{t_{i}} \notin \sigma_{i_{1}}^{\delta_{1}} \cdots \sigma_{i_{s}}^{\delta_{s}}(\mathfrak{m})$ e $\tilde{t}_{i} \notin \mathfrak{n}, \operatorname{logo} \sigma_{i_{1}}^{\delta_{1}} \cdots \sigma_{i_{s}}^{\delta_{s}}(\mathfrak{m}) \sim \mathfrak{n}$, portanto são isomorfos), e $\delta_{i}=0$, caso contrário, ou seja $i \in \underline{I}^{c}$ (pois $\tilde{t}_{i} \notin \mathfrak{m}$, o que implica que $\tilde{t_{i}} \notin \sigma_{i_{1}}^{\delta_{1}} \cdots \sigma_{i_{s}}^{\delta_{s}}(\mathfrak{m})$ e $\tilde{t_{i}} \notin \mathfrak{n}$, visto que $\mathfrak{m}$ é break maximal, e novamente $\sigma_{i_{1}}^{\delta_{1}} \cdots \sigma_{i_{s}}^{\delta_{s}}(\mathfrak{m}) \sim$ $\mathfrak{n})$.

No caso não degenerado, $\mathfrak{B}_{\mathcal{O}}=\{\mathfrak{m}\}$ e todo elemento de $\mathcal{O}$ é isomorfo a $\mathfrak{m}$ pelo Lema 3.1.1. De fato, $\underline{I}=\emptyset$, então $\tilde{t_{i}} \notin \mathfrak{m}$, para todo $i$. Assim $\sigma_{i}(\mathfrak{m}) \sim \mathfrak{m}$, isto é, $\sigma_{i}(\mathfrak{m}) \cong \mathfrak{m}$ em $\mathcal{C}_{\mathcal{O}}$, para todo $i$. Concluímos então que qualquer objeto $\mathfrak{n} \in \mathcal{O}$ será isomorfo a $\mathfrak{m} \in \mathfrak{B}_{\mathcal{O}}$. 
Portanto, em ambos os casos ( $\mathcal{O}$ degenerada ou não degenerada), $\mathcal{S}_{\mathcal{O}}$ é um esqueleto de $\mathcal{C}_{\mathcal{O}}$, pois os objetos de $\mathcal{S}_{\mathcal{O}}$ (os elementos de $\mathfrak{B}_{\mathcal{O}}$ ) não são isomorfos tomados dois a dois e cada objeto de $\mathcal{C}_{\mathcal{O}}$ (os elementos da órbita $\mathcal{O}$ ) é isomorfo a um objeto de $\mathcal{S}_{\mathcal{O}}$.

\section{Descrição algébrica do esqueleto $\mathcal{S}_{\mathcal{O}}$}

Para um dado corpo $\mathbb{F}$ e um subconjunto arbitrário $I$ do conjunto $\mathbb{N}$ dos inteiros positivos, definimos a categoria $\mathcal{A}=\mathcal{A}(\mathbb{F}, I)$ como a categoria $\mathbb{F}$-linear com conjunto de objetos $O b(\mathcal{A})$ $:=\{0,1\}^{|I|}$ (o qual assumimos ter somente uma quantidade finita de componentes não nulas quando $|I|=\infty$ ) gerada (sobre $\mathbb{F}$ ) pelo conjunto de morfismos, $\mathcal{A}_{1}:=\left\{a_{\alpha, i}, b_{\alpha, i} \mid \alpha \in\right.$ $\left.O b(\mathcal{A}), i \in I, \alpha_{i}=0\right\}$, em que $a_{\alpha, i}: \alpha \rightarrow \beta$ e $b_{\alpha, i}: \beta \rightarrow \alpha$, tais que $\beta_{j}=\alpha_{j}$ para todo $j \neq i$ e $\beta_{i}=1$, sujeitos às relações:

- $a_{\alpha, i} b_{\alpha, i}=b_{\alpha, i} a_{\alpha, i}=0$, para todos $a_{\alpha, i}, b_{\alpha, i} \in \mathcal{A}_{1}$;

- $u_{\alpha, i} v_{\beta, j}-v_{\gamma, j} u_{\delta, i}=0$, para todo $j \neq i$ e todos os possíveis $u, v \in\{a, b\}, \alpha, \beta, \gamma, \delta \in$ $O b(\mathcal{A})$, para os quais a última igualdade faça sentido.

Quando $I$ é vazio, seja $\mathcal{A}(\mathbb{F}, \emptyset)$ a categoria com um único objeto $\omega$, e com conjunto de morfismos $\mathbb{F} 1_{\omega}$.

A $\mathbb{F}$-álgebra correspondente à categoria $\mathcal{A}(\mathbb{F}, I)$ consiste de combinações $\mathbb{F}$-lineares finitas de morfismos na categoria, e o produto é simplesmente a composição dos morfismos sempre que é definido e é 0 caso contrário. Ela tem um elemento unidade se $I$ é finito. Vamos adotar a mesma notação $\mathcal{A}(\mathbb{F}, I)$ para a álgebra. A álgebra $\mathcal{A}(\mathbb{F}, I)$ tem dimensão finita quando $|I|=$ $s<\infty$, como por exemplo $I=\left\{i_{1}, \ldots, i_{s}\right\}$, em que $\mathcal{A}(\mathbb{F}, I) \cong \mathcal{A}\left(\mathbb{F},\left\{i_{1}\right\}\right) \otimes \cdots \otimes \mathcal{A}\left(\mathbb{F},\left\{i_{s}\right\}\right)$. A álgebra $\mathcal{A}(\mathbb{F},\{i\})$ é isomorfa à álgebra $\overline{\mathcal{Q}_{1}}:=\mathbb{F} \mathcal{Q}_{1} / \mathcal{R}$ correspondendo ao seguinte quiver e relações:

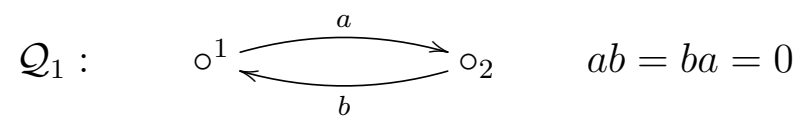

Como $\overline{\mathcal{Q}_{1}}$ é gerada sobre $\mathbb{F}$ por $1_{1}, 1_{2}, a, b$, módulo as relações $a b=0=b a$, ela tem dimensão 4 (observamos que existem as relações óbvias: $a^{2}=0,1_{1}^{2}=1_{1}, 1_{2}^{2}=1_{2}, b^{2}=0$, $a 1_{1}=a, 1_{2} a=a, 1_{1} b=b$ e $\left.b 1_{2}=b\right)$.

Para subconjuntos $I$ e $J$ de $\mathbb{N}$ e uma $I \cup J$-upla $\underline{\tau}=\left(\tau_{i}\right)_{i \in I \cup J}$ de automorfismos comutantes de $\mathbb{F}$, seja $\mathcal{B}(\mathbb{F}, I, J, \tau)$ a $\mathbb{F}$-categoria com um único objeto, $\omega$, cuja álgebra dos endomorfismos é uma álgebra associativa unital sobre $\mathbb{F}$ gerada por $a_{i}, b_{i}, c_{j}, c_{j}^{-1}, i \in I, j \in J$, sujeitos às relações: $a_{i} b_{i}=b_{i} a_{i}=0, a_{i} \lambda=\tau_{i}(\lambda) a_{i}, \lambda b_{i}=b_{i} \tau_{i}(\lambda)$, para $i \in I$ e $\lambda \in \mathbb{F}, c_{j} c_{j}^{-1}=1=1_{\omega}$, $c_{j} \lambda=\tau_{j}(\lambda) c_{j}$, para $j \in J$ e $\lambda \in \mathbb{F}$, e $u_{s} v_{t}=v_{t} u_{s}$, para todos $u, v \in\{a, b, c\}$ e todos $s, t \in$ $I \cup J$.

Proposição 3.1.2. Seja $A=A_{n}$ a n-ésima álgebra de Weyl e vamos assumir que $\mathcal{O}$ é uma órbita em $D=k\left[\tilde{t_{1}}, \ldots, \tilde{t_{n}}\right]$ sob o grupo de automorfismos $\mathcal{G}$ (se $\mathcal{O}$ é degenerada $e$ $n=\infty$, vamos assumir que $\mathcal{O}$ tem break maximal). Seja $\mathfrak{m}$ o único elemento de $\mathfrak{B}_{\mathcal{O}}$ no caso não degenerado, e o break maximal de $\mathcal{O}$ utilizado na definição da categoria $\mathcal{C}_{\mathcal{O}}$, no caso degenerado.

1. Se $\operatorname{char}(k)=0$, então $\mathcal{S}_{\mathcal{O}} \cong \mathcal{A}(D / \mathfrak{m}, \underline{I}(\mathfrak{m}))$;

2. Se $\operatorname{char}(k)=p>0$, então $\mathcal{S}_{\mathcal{O}} \cong \mathcal{B}\left(D / \mathfrak{m}, \underline{I}(\mathfrak{m}), \underline{I}(\mathfrak{m})^{c}, \underline{\tau}(\mathfrak{m})\right)$.

Demonstração. O Corolário 3.1.1 mostra que a categoria $\mathcal{S}_{\mathcal{O}}$ com objetos $\mathfrak{B}_{\mathcal{O}}$ é um esqueleto de $\mathcal{C}_{\mathcal{O}}$. 
Vamos assumir que $\operatorname{char}(k)=0$. Quando $\underline{I}(\mathfrak{m}) \neq \emptyset$, definimos o funtor $G: \mathcal{A}(D / \mathfrak{m}, \underline{I}(\mathfrak{m}))$ $\rightarrow \mathcal{S}_{\mathcal{O}}$ como segue:

$$
G(\alpha)=\sigma^{\alpha}(\mathfrak{m}) \quad, \quad G\left(a_{\alpha, i}\right)=X_{\sigma^{\alpha}(\mathfrak{m}), i} \quad, \quad G\left(b_{\alpha, i}\right)=Y_{\sigma^{\alpha}(\mathfrak{m}), i}
$$

em que $\sigma^{\alpha}=\prod_{i} \sigma_{i}^{\alpha_{i}}$. Observamos que $\sigma^{\alpha}(\mathfrak{m})=\sigma_{i_{1}}^{\alpha_{1}} \cdots \sigma_{i_{s}}^{\alpha_{s}}(\mathfrak{m}) \in \mathfrak{B}_{\mathcal{O}}=\operatorname{Ob}\left(\mathcal{S}_{\mathcal{O}}\right)$, em que $i_{1}, \ldots, i_{s} \in \underline{I}(\mathfrak{m})$. Como $\mathcal{S}_{\mathcal{O}}$ é um esqueleto de $\mathcal{C}_{\mathcal{O}}$, temos que $\mathcal{S}_{\mathcal{O}} \subseteq \mathcal{C}_{\mathcal{O}}$ (é uma subcategoria plena de $\mathcal{C}_{\mathcal{O}}$ ). Pela definição da categoria $\mathcal{C}_{\mathcal{O}}$, os morfismos em $\mathcal{S}_{\mathcal{O}}$ são da forma $X_{\sigma^{\alpha}(\mathfrak{m}), i}$ e $Y_{\sigma^{\alpha}(\mathfrak{m}), i}$, em que $\sigma^{\alpha}(\mathfrak{m}) \in \mathfrak{B}_{\mathcal{O}}$ e $i \in \underline{I}(\mathfrak{m})$. Assim podemos definir um funtor $G^{\prime}$ de maneira natural, que é o inverso de $G$, e segue que $G$ é um isomorfismo. Agora vamos supor que $\underline{I}(\mathfrak{m})=\emptyset$. Logo, a órbita é não degenerada. Observamos que no caso em que $\underline{I}(\mathfrak{m})=\emptyset$, a categoria $\mathcal{A}(D / \mathfrak{m}, \emptyset)$ possui um único objeto $\omega$ com conjunto de morfismos $\mathbb{F} 1_{\omega}$ e no caso em que a órbita é não degenerada, $\mathfrak{B}_{\mathcal{O}}=O b\left(\mathcal{S}_{\mathcal{O}}\right)$ também possui um único objeto $\mathfrak{m}$. Então temos o funtor $G: \mathcal{A}(D / \mathfrak{m}, \emptyset) \rightarrow \mathcal{S}_{\mathcal{O}}$ definido por:

$$
G(\omega)=\mathfrak{m} \quad, \quad G\left(1_{\omega}\right)=1_{\mathfrak{m}}
$$

que é um isomorfismo.

Agora, vamos supor que $\operatorname{char}(k)=p>0$, e para $1 \leq i \leq n, r_{i}$ denotará o menor inteiro positivo tal que $\sigma_{i}^{r_{i}}(\mathfrak{m})=\mathfrak{m}$. Então, $r_{i}=p$ ou 1 .

Assim definimos um funtor $G: \mathcal{B}\left(D / \mathfrak{m}, \underline{I}(\mathfrak{m}), \underline{I}(\mathfrak{m})^{c}, \underline{\tau}(\mathfrak{m})\right) \rightarrow \mathcal{S}_{\mathcal{O}}$ da seguinte maneira:

$$
\begin{gathered}
G(\omega)=\mathfrak{m} \\
G\left(a_{i}\right)=X_{\sigma_{i}^{r_{i}-1}(\mathfrak{m}), i} X_{\sigma_{i}^{r_{i}-2}(\mathfrak{m}), i} \cdots X_{\sigma_{i}(\mathfrak{m}), i} X_{\mathfrak{m}, i} \\
G\left(b_{i}\right)=Y_{\mathfrak{m}, i} Y_{\sigma_{i}(\mathfrak{m}), i} \cdots Y_{\sigma_{i}^{r_{i}-2}(\mathfrak{m}), i} Y_{\sigma_{i}^{r_{i}-1}(\mathfrak{m}), i} \\
G\left(c_{j}\right)=X_{\sigma_{j}^{r_{j}-1}(\mathfrak{m}), j} X_{\sigma_{j}^{r_{j}-2}(\mathfrak{m}), j} \cdots X_{\sigma_{j}(\mathfrak{m}), j} X_{\mathfrak{m}, j}
\end{gathered}
$$

Por conta das relações satisfeitas pelas categorias, $G$ é um isomorfismo.

Observação 3.1.4. As álgebras $\mathcal{A}(D / \mathfrak{m}, \underline{I}(\mathfrak{m}))$ possuem dimensão finita quando $|\underline{I}(\mathfrak{m})|<$ $\infty$; enquanto que as álgebras $\mathcal{B}\left(D / \mathfrak{m}, \underline{I}(\mathfrak{m}), \underline{I}(\mathfrak{m})^{c}, \underline{\tau}(\mathfrak{m})\right)$ sempre possuem dimensão infinita.

\subsubsection{Descrição dos módulos de peso simples}

Nesta subseção vamos descrever os módulos de peso simples para a álgebra de Weyl $A=A_{n} \operatorname{com} 1 \leq n \leq \infty$ (em que $A$ é uma realização da $n$-ésima álgebra de Weyl $A_{n}$ como a álgebra de Weyl generalizada $D(\underline{\sigma}, \underline{a})$ cf. Capítulo 1, Seção 1.4). Estes resultados generalizam aqueles obtidos em [BB00], em que o corpo subjacente era algebricamente fechado. $\mathrm{O}$ caso da primeira álgebra de Weyl sobre um corpo arbitrário foi tratado previamente em, por exemplo, [DGO96], [Bav92a], [Bav92b] e [BvO97].

A descrição dos módulos de peso para a álgebra de Weyl $A$ será feita via redução à classe de categorias lineares. Embora essas categorias pareçam mais complicadas do que álgebras, pela necessidade de lidar com muitos objetos, elas nos oferecem uma certa comodidade, que as vezes é decisiva para os cálculos, mas muitas vezes para a compreensão também.

Se o corpo tem característica 0, pela Proposição 3.1.2, podemos concentrar a análise em álgebras da forma $\mathcal{A}(\mathbb{F}, I)$.

Então, vamos assumir que $\mathbb{F}$ é um corpo com característica 0. Para cada $\alpha \in O b(\mathcal{A}(\mathbb{F}, I))$, definimos um $\mathcal{A}(\mathbb{F}, I)$-módulo simples $S_{\alpha}$ tal que $S_{\alpha}(\beta)=\delta_{\alpha \beta} \mathbb{F}$, em que $\delta_{\alpha \beta}$ é o delta de Kronecker, para todos os objetos $\beta \in O b(\mathcal{A}(\mathbb{F}, I))$, e todos os morfismos são triviais. 
Para lidar com o caso $n=\infty$ precisamos do seguinte resultado conhecido, que pode ser encontrado em [DOF94].

Lema 3.1.2. Seja $\mathcal{C}$ uma categoria, assuma que $M$ é um $\mathcal{C}$-módulo simples e que $M(\alpha) \neq\{0\}$ para algum $\alpha \in O b(\mathcal{C})$. Então $M(\alpha)$ é simples como um $\mathcal{C}(\alpha, \alpha)$-módulo. Reciprocamente, para qualquer $\mathcal{C}(\alpha, \alpha)$-módulo simples $N$, existe um único (a menos de isomorfismo) $\mathcal{C}$ módulo simples $M$ tal que $M(\alpha) \cong N$ como $\mathcal{C}(\alpha, \alpha)$-módulos.

Proposição 3.1.3. Qualquer módulo simples sobre a álgebra $\mathcal{A}=\mathcal{A}(\mathbb{F}, I)$ é isomorfo à $S_{\alpha}$ para algum objeto $\alpha \in O b(\mathcal{A}(\mathbb{F}, I))$.

Demonstração. Se $n<\infty$ e $I \subseteq\{1, \ldots, n\}$, então a afirmação é clara pois $\mathcal{A}(\mathbb{F}, I)$ tem dimensão finita. Suponhamos que $n=\infty$, e seja $M$ um $\mathcal{A}$-módulo simples. Para um objeto $\alpha \in O b(\mathcal{A})$ denotaremos por $M(\alpha)$, o módulo $M$ em $\alpha$. Vamos assumir que $M(\alpha) \neq\{0\}$. Pelo Lema 3.1.2, $M(\alpha)$ é um $\mathcal{A}(\alpha, \alpha)$-módulo simples. Suponhamos que $u \in \mathcal{A}(\alpha, \alpha), u \neq 0,1_{\alpha}$, e $u=\left(u_{1}\right)_{\beta^{1}, i_{1}} \cdots\left(u_{m}\right)_{\beta^{m}, i_{m}}$ em que $u_{j} \in\{a, b\}, \beta^{j} \in O b(\mathcal{A})$, e $i_{j} \in I$ para cada $j=1, \ldots, m$. Como $u \in \mathcal{A}(\alpha, \alpha)$, então para cada $j$, existe $k$ tal que $\left\{u_{j}, u_{k}\right\}=\{a, b\}$ e $i_{j}=i_{k}$. Usando o segundo conjunto de relações (as relações comutantes) em $\mathcal{A}(\mathbb{F}, I)$, podemos reescrever $u$ como o produto $u=\prod_{j} a_{\beta^{j}, s_{j}} b_{\beta^{j}, s_{j}}$. Mas então, pelo primeiro conjunto de relações em $\mathcal{A}(\mathbb{F}, I)$, temos que $u=0$, o que contradiz nossa suposição. Portanto, $\mathcal{A}(\alpha, \alpha)=\mathbb{F} 1_{\alpha}$. Como $M(\alpha)$ é um $\mathcal{A}(\alpha, \alpha)$-módulo simples, ou seja, $M(\alpha)$ é $\mathbb{F} 1_{\alpha}$-módulo simples, ele é unidimensional.

Consideremos o conjunto $J$ de todo $\beta \in O b(\mathcal{A})$ tal que $\alpha_{j}=\beta_{j}$ para todo $j \in I$ exceto um e $M(\beta) \neq\{0\}$. Quando $\beta \in J$ e $\beta_{i} \neq \alpha_{i}$, dizemos que $\beta$ é um $\alpha$ - sink se $a_{\alpha, i} \neq 0$ (então $b_{\alpha, i}=0$, pois $a_{\alpha, i} b_{\alpha, i}=b_{\alpha, i} a_{\alpha, i}=0$ ) e é um $\alpha$-source se $b_{\alpha, i} \neq 0$ (então $a_{\alpha, i}=0$, pois $a_{\alpha, i} b_{\alpha, i}=b_{\alpha, i} a_{\alpha, i}=0$ ). Seja $J_{1}$ (resp. $J_{2}$ ) o conjunto de todos $\alpha$-sinks em $J$ (resp. o conjunto de todos $\alpha$-sources em $J)$ e $J_{3}=J \backslash\left(J_{1} \cup J_{2}\right)$. Vamos considerar o seguinte submódulo de $M: M^{\prime}=\mathcal{A}\left(\sum_{\beta \in J_{1} \cup J_{3}} M(\beta)\right)$. Afirmamos que $M^{\prime} \cap M(\alpha)=\{0\}$. De fato, se $u \in \mathcal{A}$ e $u M(\beta)=M(\alpha)$ para algum $\beta \in J_{1} \cup J_{3}$, então segue das relações comutantes que $u$ contém $b_{\alpha, i}$, em que $\beta_{i}=1$ e $\beta_{j}=\alpha_{j}$ para $j \neq i$. Mas como $\beta \in J_{1} \cup J_{3}$, ele deve ser tal que $b_{\alpha, i}=0$, e então $u=0$. Concluímos que $M^{\prime} \cap M(\alpha)=\{0\}$, logo $M^{\prime}=\{0\}$ pela simplicidade de $M(\alpha)$. Este argumento mostra que sempre que $M(\alpha) \neq\{0\}$, então ele não tem nenhum $\alpha$-sinks. Mas então, pela simplicidade, devemos ter $M=M(\alpha)$, e portanto $M=S_{\alpha}$ neste caso.

Se o corpo tem característica $p>0$, pela Proposição 3.1.2, é suficiente considerar as álgebras $\mathcal{B}(\mathbb{F}, I, J, \underline{\tau})$ em que $I, J \subseteq \mathbb{N}$.

Vamos assumir $\Gamma \subseteq I$ e $\xi: \Gamma \rightarrow\{0,1\}$. Temos a seguinte subálgebra de $\mathcal{B}(\mathbb{F}, I, J, \underline{\tau})$, associada a estes dados,

$$
\mathfrak{R}_{\Gamma, \xi}=\mathbb{F}\left[d_{i}, c_{j}^{ \pm 1}, \underline{\tau} \mid i \in \Gamma, j \in J\right]
$$

em que $d_{i}=a_{i}$ se $\xi(i)=0$ e $d_{i}=b_{i}$ se $\xi(i)=1$ (na subálgebra somente os automorfismos $\tau_{i}$ com $i \in \Gamma \cup J$ são usados, e $\mathfrak{R}_{\Gamma, \xi}$ é uma álgebra dos polinômios torcida - veja Seção 3.4). Então para um ideal maximal $\mathfrak{N}$ em $\mathfrak{R}_{\Gamma, \xi}$, definimos o módulo simples $S_{\Gamma, \xi, \mathfrak{N}}=\mathfrak{R}_{\Gamma, \xi} / \mathfrak{N}$. Notemos que quando $I=\emptyset$, então $\Gamma=\emptyset$; e sempre que $\Gamma=\emptyset$, vamos assumir que existe apenas um $\xi$ e $\mathfrak{R}_{\Gamma, \xi}:=\mathbb{F}\left[c_{j}^{ \pm 1}, \underline{\tau} \mid j \in J\right]$. Novamente neste caso, para todo ideal maximal $\mathfrak{N}$ de $\mathfrak{R}_{\Gamma, \xi}$, definimos $S_{\Gamma, \xi, \mathfrak{N}}=\mathfrak{R}_{\Gamma, \xi} / \mathfrak{N}$.

Proposição 3.1.4. Os módulos $S_{\Gamma, \xi, \mathfrak{N}}$ constituem uma lista exaustiva de $\mathcal{B}(\mathbb{F}, I, J, \underline{\tau})$-módulos simples não isomorfos tomados dois a dois.

Demonstração. Como o caso $I=\emptyset$ é natural conforme descrito acima, podemos assumir que $S$ é um $\mathcal{B}(\mathbb{F}, I, J, \underline{\tau})$-módulo simples em que $I \neq \emptyset$. 
Como $\mathcal{B}(\mathbb{F}, I, J, \underline{\tau})$ possui um único objeto $\omega$, podemos dizer que $S=S(\omega)$. Então, $a_{i} u \in$ $S$ e $b_{i} u \in S$, para todos $a_{i}, b_{i} \in \mathcal{B}(\mathbb{F}, I, J, \underline{\tau})(\omega, \omega)$ e $u \in S$, ou seja, $a_{i} S \subseteq S$ e $b_{i} S \subseteq S$. Além disso, para qualquer morfismo $d \in \mathcal{B}(\mathbb{F}, I, J, \underline{\tau})(\omega, \omega)$ e qualquer $u \in S$ temos que, $d a_{i} u \in a_{i} S$ e $d b_{i} u \in b_{i} S$, por conta das relações satisfeitas pelos morfismos na categoria $\mathcal{B}(\mathbb{F}, I, J, \underline{\tau})$. Logo $a_{i} S$ e $b_{i} S$ são $\mathcal{B}(\mathbb{F}, I, J, \underline{\tau})$-submódulos de $S$, para todo $i \in I$.

Como $S$ é simples, cada um deles coincide com $S$ ou com $\{0\}$. Mas $a_{i} S=S$ implica $b_{i} S=b_{i} a_{i} S=\{0\}$ e vice versa. Portanto, ou $a_{i} S=b_{i} S=\{0\}$, ou $a_{i} S=S$ e $b_{i} S=\{0\}$, ou $a_{i} S=\{0\}$ e $b_{i} S=S$, para qualquer $i \in I$. Seja $\Gamma=\left\{i \in I \mid\right.$ ou $a_{i} S \neq\{0\}$ ou $\left.b_{i} S \neq\{0\}\right\}$. Definimos $\xi: \Gamma \rightarrow\{0,1\}$ por $\xi(i)=0$ se $a_{i} S \neq\{0\}, \xi(i)=1$ se $b_{i} S \neq\{0\}$. Então $\mathcal{B}(\mathbb{F}, I, J, \underline{\tau})$ módulo o aniquilador de $S$ em $\mathbb{F}\left[a_{i}, b_{i}, \underline{\tau} \mid i \in I\right]$ é isomorfo à $\mathfrak{R}_{\Gamma, \xi}$. O resultado segue.

Agora vamos descrever os módulos simples para a álgebra de Weyl $A=A_{n}$ de acordo com as Proposições 3.1.3 e 3.1.4. Como antes, vamos assumir $D=k\left[\tilde{t}_{1}, \ldots, \tilde{t_{n}}\right]$ em que $\tilde{t}_{i}=\partial_{i} x_{i}$. Posteriormente descreveremos os módulos de peso simples para a álgebra $A$, que é o propósito desta seção.

Vamos assumir primeiramente que $\operatorname{char}(k)=0$ e que $\mathcal{O}$ é uma órbita não degenerada de $\mathcal{G}$ em $\mathfrak{m a x} D$. Neste caso, $\mathfrak{B}_{\mathcal{O}}=\{\mathfrak{m}\}$. Seja

$$
S(\mathcal{O})=\bigoplus_{\mathfrak{n} \in \mathcal{O}} D / \mathfrak{n}
$$

e definimos uma estrutura de $A$-módulo à esquerda em $S(\mathcal{O})$ especificando para $i=1, \ldots, n$ :

$$
x_{i}(d+\mathfrak{n}):=\sigma_{i}(d)+\sigma_{i}(\mathfrak{n}) \quad, \quad \partial_{i}(d+\mathfrak{n}):=\tilde{t}_{i} \sigma_{i}^{-1}(d)+\sigma_{i}^{-1}(\mathfrak{n}) .
$$

Como $S(\mathcal{O})$ é gerado por $1+\mathfrak{m}$, pois cada elemento da órbita $\mathcal{O}$ é isomorfo a $\mathfrak{m}$, temos que $S(\mathcal{O}) \cong A / A \mathfrak{m}$, em que $1+\mathfrak{m} \mapsto 1+A \mathfrak{m}$.

Agora vamos supor que $\operatorname{char}(k)=0, \mathcal{O}$ é degenerada, e $\mathfrak{m}$ é o break maximal fixado. Para $\mathfrak{p} \in \mathfrak{B}_{\mathcal{O}}$ seja

$$
S(\mathcal{O}, \mathfrak{p}):=\bigoplus_{\mathfrak{n} \in \mathcal{O}_{\mathfrak{p}}} D / \mathfrak{n}
$$

Podemos definir uma estrutura de $A$-módulo à esquerda em $S(\mathcal{O}, \mathfrak{p})$ da mesma forma que definimos acima $($ em $S(\mathcal{O}))$, mas quando a imagem não está em $S(\mathcal{O}, \mathfrak{p})$, o resultado é 0 . Assumindo $\mathfrak{p}=\sigma_{i_{1}}^{\delta_{1}} \cdots \sigma_{i_{s}}^{\delta_{s}}(\mathfrak{m})$, em que $\underline{I}(\mathfrak{m})=\left\{i_{1}, \ldots, i_{s}\right\}$, temos neste caso $S(\mathcal{O}, \mathfrak{p}) \cong$ $A / A\left(\mathfrak{p}, Z_{i_{1}}, \ldots, Z_{i_{s}}\right)$, tal que $Z_{k}=x_{k}$ se $\mathfrak{p}$ é um break com respeito a $k$ (ou seja, se $\delta_{k}=0$ ), e $Z_{k}=\partial_{k}$, caso contrário. O isomorfismo é dado por $1+\mathfrak{p} \mapsto 1+A\left(\mathfrak{p}, Z_{i_{1}}, \ldots, Z_{i_{s}}\right)$. Segue da construção que $S(\mathcal{O})$ e $S(\mathcal{O}, \mathfrak{p})$ são $A$-módulos simples.

Suponhamos agora que $\operatorname{char}(k)=p>0$. Seja $\mathfrak{m}$ o ideal maximal designado quando a órbita $\mathcal{O}$ é não degenerada, e seja $\mathfrak{m}$ o break maximal fixado quando $\mathcal{O}$ é degenerada. Vamos assumir que $\underline{I}=\underline{I}(\mathfrak{m})$ é o conjunto de breaks de $\mathfrak{m}$, e seja $\Gamma$ um subconjunto de $\underline{I}$. Escolha $\xi: \Gamma \rightarrow\{0,1\}$. Então para $\mathfrak{n} \in \mathcal{O}$, definimos

$$
\mathfrak{R}_{\Gamma, \xi}(\mathfrak{n})=(D / \mathfrak{n})\left[d_{i}, c_{j}^{ \pm 1}, \underline{\tau}(\mathfrak{n}) \mid i \in \Gamma, j \in \underline{I}^{c}\right],
$$

em que $d_{i}:=a_{i}$ se $\xi(i)=0$ e $d_{i}:=b_{i}$ se $\xi(i)=1$. Notemos que se $\Gamma=\emptyset$, então $\mathfrak{R}_{\Gamma, \xi}(\mathfrak{n})=$ $(D / \mathfrak{n})\left[c_{j}^{ \pm 1}, \underline{\tau}(\mathfrak{n}) \mid j=1, \ldots, n\right]$ (e existe somente um $\xi$ possível). Seja $\mathfrak{N}$ um ideal maximal no anel $\mathfrak{R}_{\Gamma, \xi}(\mathfrak{m})$. Seja

$$
S(\mathcal{O}, \Gamma, \xi, \mathfrak{N}):=\bigoplus_{\mathfrak{n} \in \mathcal{O}} \mathfrak{R}_{\Gamma, \xi}(\mathfrak{n}) / \sigma_{\mathfrak{n}}(\mathfrak{N})
$$

em que $\sigma_{\mathfrak{n}}(\mathfrak{N})$ significa aplicar $\sigma_{\mathfrak{n}}$ nos coeficientes dos elementos de $\mathfrak{N}$ (lembrando que 
$\left.\sigma_{\mathfrak{n}}: D / \mathfrak{m} \rightarrow D / \mathfrak{n}\right)$. Definimos uma estrutura de um $A$-módulo à esquerda em $S(\mathcal{O}, \Gamma, \xi, \mathfrak{N})$ especificando para cada $i \in\{1,2, \ldots, n\}$,

$x_{i}\left(f+\sigma_{\mathfrak{n}}(\mathfrak{N})\right)= \begin{cases}\sigma_{i}(f)+\sigma_{i} \sigma_{\mathfrak{n}}(\mathfrak{N}) & \text { se } \mathfrak{n} \neq \sigma \mathfrak{m} \text { para qualquer } \sigma \in \widehat{\mathcal{G}}_{i} ; \\ b_{i} \sigma_{i} \sigma_{\mathfrak{n}}(f)+\sigma_{i} \sigma_{\mathfrak{n}}(\mathfrak{N}) & \text { se } \mathfrak{n}=\sigma \mathfrak{m} \text { para algum } \sigma \in \widehat{\mathcal{G}}_{i}, i \in \Gamma \text { e } \xi(i)=1 \\ c_{i} \sigma_{i} \sigma_{\mathfrak{n}}(f)+\sigma_{i} \sigma_{\mathfrak{n}}(\mathfrak{N}) & \text { se } \mathfrak{n}=\sigma \mathfrak{m} \text { para algum } \sigma \in \widehat{\mathcal{G}_{i}} \text { e } i \in \underline{I}(\mathfrak{m})^{c} ; \\ 0 & \text { em todos os outros casos. }\end{cases}$

$\partial_{i}\left(f+\sigma_{\mathfrak{n}}(\mathfrak{N})\right)= \begin{cases}\tilde{t}_{i} \sigma_{i}^{-1} \sigma_{\mathfrak{n}}(f)+\sigma_{i}^{-1} \sigma_{\mathfrak{n}}(\mathfrak{N}) & \text { se } \mathfrak{n} \neq \sigma_{i} \sigma \mathfrak{m} \text { para qualquer } \sigma \in \widehat{\mathcal{G}}_{i} ; \\ a_{i} \tilde{t_{i}} \sigma_{i}^{-1} \sigma_{\mathfrak{n}}(f)+\sigma_{i}^{-1} \sigma_{\mathfrak{n}}(\mathfrak{N}) & \text { se } \mathfrak{n}=\sigma_{i} \sigma \mathfrak{m} \text { para algum } \sigma \in \widehat{\mathcal{G}}_{i}, i \in \Gamma \\ & \text { e } \xi(i)=0 ; \\ c_{i}^{-1} \tilde{t}_{i} \sigma_{i}^{-1} \sigma_{\mathfrak{n}}(f)+\sigma_{i}^{-1} \sigma_{\mathfrak{n}}(\mathfrak{N}) & \text { se } \mathfrak{n}=\sigma_{i} \sigma \mathfrak{m} \text { para algum } \sigma \in \widehat{\mathcal{G}}_{i} \text { e } i \in \underline{I}(\mathfrak{m})^{c} \\ 0 & \text { em todos os outros casos. }\end{cases}$

em que $\widehat{\mathcal{G}_{i}}$ é o subgrupo de $\mathcal{G}$ gerado por $\sigma_{j}$ para todos $j \neq i$. Segue da construção que $S(\mathcal{O}, \Gamma, \xi, \mathfrak{N})$ é um $A$-módulo simples.

O próximo teorema fornece uma classificação dos $A_{n}$-módulos de peso simples, em que $1 \leq n \leq \infty$.

Teorema 3.1.1. Seja $A_{n}$ a n-ésima álgebra de Weyl para $1 \leq n \leq \infty$ sobre um corpo $k$,

1. Se char $(k)=0$, então os módulos $S(\mathcal{O})$ e $S(\mathcal{O}, \mathfrak{p})$, em que $\mathfrak{p} \in \mathfrak{B}_{\mathcal{O}}$, constituem uma lista exaustiva de $A_{n}$-módulos de peso simples não isomorfos, tomados dois a dois, com suporte em $\mathcal{O}$.

2. Se char $(k)=p>0$, então os módulos $S(\mathcal{O}, \Gamma, \xi, \mathfrak{N})$ constituem uma lista exaustiva de $A_{n}$-módulos de peso simples não isomorfos, tomados dois a dois, com suporte $\mathcal{O}$.

(Em 1. e 2., assume-se que a órbita $\mathcal{O}$ tem um break maximal quando $\mathcal{O}$ é degenerada e $n=\infty$.)

Demonstração. Fixemos uma órbita $\mathcal{O}$ de $D=k\left[\tilde{t_{1}}, \ldots, \tilde{t_{n}}\right]$ sob o grupo $\mathcal{G}$ e seja $\mathcal{C}_{\mathcal{O}}$ e $\mathcal{S}_{\mathcal{O}}$ a categoria e o esqueleto, respectivamente, associados. Podemos identificar $\mathcal{S}_{\mathcal{O}}$ com uma álgebra $\mathcal{A}(D / \mathfrak{m}, \underline{I}(\mathfrak{m}))$ ou $\mathcal{B}\left(D / \mathfrak{m}, \underline{I}(\mathfrak{m}), \underline{I}(\mathfrak{m})^{c}, \underline{\tau}(\mathfrak{m})\right)$ usando o funtor $G$ definido na demonstração da Proposição 3.1.2. Vamos usar a determinação dos módulos para essas álgebras através das Proposições 3.1.3 e 3.1.4. Denotaremos por $E$ o funtor equivalência $\mathcal{C}_{\mathcal{O}} \otimes_{\mathcal{S}_{\mathcal{O}}}-: \mathcal{S}_{\mathcal{O}^{-}} \bmod \rightarrow \mathcal{C}_{\mathcal{O}^{-}} \bmod$ e seja $F^{\prime}: \mathcal{C}_{\mathcal{O}^{-}} \bmod \rightarrow \mathcal{W}_{\mathcal{O}}(A)$ dado na demonstração da Proposição 3.1.1.

1. $\operatorname{char}(k)=0$ : Quando a órbita $\mathcal{O}$ é não degenerada, temos que $\underline{I}(\mathfrak{m})=\emptyset$ e $\mathcal{A}(D / \mathfrak{m}, \emptyset)$ possui um único elemento $\omega$. O funtor $G$ identifica $\mathcal{S}_{\mathcal{O}} \operatorname{com} \mathcal{A}(D / \mathfrak{m}, \emptyset)$, então podemos pensar que o $\mathcal{A}(D / \mathfrak{m}, \emptyset)$-módulo simples $S_{\omega}$ é um $\mathcal{S}_{\mathcal{O}}$-módulo simples. Temos que $F^{\prime} E G: \mathcal{A}(D / \mathfrak{m}, \emptyset)$-mod $\rightarrow \mathcal{W}_{\mathcal{O}}(A)$, assim $F^{\prime} E G\left(S_{\omega}\right) \in \mathcal{W}_{\mathcal{O}}(A)$ é um $A$-módulo de peso simples. É fácil ver que $F^{\prime} E G\left(S_{\omega}\right) \cong S(\mathcal{O})$. Quando a órbita é degenerada, e $\mathfrak{m}$ é o break maximal designado com conjunto de breaks $\underline{I}(\mathfrak{m})$, temos para cada $\alpha \in$ $\mathcal{A}(D / \mathfrak{m}, \underline{I}(\mathfrak{m}))$ que $F^{\prime} E G\left(S_{\alpha}\right) \cong S\left(\mathcal{O}, \sigma^{\alpha}(\mathfrak{m})\right)$, em que $\sigma^{\alpha}=\prod_{i \in \underline{I}(\mathfrak{m})} \sigma_{i}^{\alpha_{i}}$. 
2. $\operatorname{char}(k)=p>0$ : Utilizando o funtor $G$ podemos identificar $\mathcal{B}\left(D / \mathfrak{m}, \underline{I}(\mathfrak{m}), \underline{I}(\mathfrak{m})^{c}, \underline{\tau}(\mathfrak{m})\right)$ com $\mathcal{S}_{\mathcal{O}}$. Para o módulo simples $S_{\Gamma, \xi, \mathfrak{N}}$ sobre $\mathcal{B}\left(D / \mathfrak{m}, \underline{I}(\mathfrak{m}), \underline{I}(\mathfrak{m})^{c}, \underline{\tau}(\mathfrak{m})\right)$ temos que $F^{\prime} E G\left(S_{\Gamma, \xi, \mathfrak{N}}\right) \cong S(\mathcal{O}, \Gamma, \xi, \mathfrak{N})$. A afirmação neste caso é uma consequência da Proposição 3.1.4.

\subsubsection{Exemplo: caso $\mathrm{A}_{1}$}

Seja $A_{1}=k[t](\sigma, t)$ a primeira álgebra de Weyl, em que $t=\partial x$ e $\sigma(t)=t-1$ (uma realização como uma álgebra de Weyl generalizada de grau 1). Os $A_{1}$-módulos de peso simples foram descritos em [Blo81], [Bav92a], [Bav92b] e [BvO97], e eles são os seguintes:

1. Vamos assumir que $\operatorname{char}(k)=0, \mathcal{O}$ é uma órbita não degenerada de $\max (k[t])$ sob $\sigma$, e $\mathfrak{m} \in \mathcal{O}$. Então $\mathfrak{m}$ é um ideal principal gerado por um polinômio irredutível diferente de $t$ sobre $k$ e $S(\mathcal{O})=A / A \mathfrak{m}$ é o correspondente $A$-módulo simples.

2. Vamos assumir que $\operatorname{char}(k)=0, \mathcal{O}$ é uma órbita degenerada, e $\mathfrak{m} \in \mathcal{O}$ é o break maximal fixado (o qual é único aqui). Então $\mathfrak{m}=\langle t\rangle, \mathfrak{B}_{\mathcal{O}}=\{\mathfrak{m}, \sigma(\mathfrak{m})\}, S(\mathcal{O}, \mathfrak{m}) \cong$ $A / A x$ e $S(\mathcal{O}, \sigma(\mathfrak{m})) \cong A / A \partial$.

3. Vamos assumir que $\operatorname{char}(k)=p>0, \mathcal{O}$ é não degenerada, e $\mathfrak{m}$ é o ideal maximal fixado em $\mathcal{O}$. Se $\sigma(\mathfrak{m}) \neq \mathfrak{m}$, então $\mathfrak{R}_{\Gamma, \xi}(\mathfrak{m})=(k[t] / \mathfrak{m})\left[c^{ \pm 1}\right]$ (aqui $\Gamma=\emptyset$ e existe apenas um $\xi)$. Então os $A$-módulos simples $S(\mathcal{O}, \emptyset, \xi, \mathfrak{N})$ são parametrizados por ideais maximais $\mathfrak{N}=\langle f\rangle$ de $(k[t] / \mathfrak{m})\left[c^{ \pm 1}\right]$ gerados por polinômios irredutíveis $f$ de $(k[t] / \mathfrak{m})[c]$ diferentes de $c$. Quando $\sigma(\mathfrak{m})=\mathfrak{m}$, (por exemplo, quando $\mathfrak{m}$ é gerado por um polinômio da forma $f(t)=t^{p}-t-\nu$ para algum $\nu \in k$ não nulo e $f$ irredutível), então $\mathfrak{R}:=\mathfrak{R}_{\Gamma, \xi}(\mathfrak{m})=$ $(k[t] / \mathfrak{m})\left[c^{ \pm 1}, \sigma\right]$ é uma álgebra polinomial torcida. Neste caso, os $A$-módulos simples $S(\mathcal{O}, \emptyset, \xi, \mathfrak{N})$ são parametrizados por ideais maximais $\mathfrak{N}$ de $\mathfrak{R}$. Qualquer ideal maximal é principal, $\mathfrak{N}=\langle f\rangle$, em que $f$ é irredutível em $\mathfrak{R}$, e $\mathfrak{R} /\langle f\rangle \cong \mathfrak{R} /\langle g\rangle$ se, e somente se, $f$ e $g$ são similares. (veja por exemplo, [DGO96].)

4. Vamos assumir que $\operatorname{char}(k)=p>0, \mathcal{O}$ é degenerada, e $\mathfrak{m} \in \mathcal{O}$ é o break maximal fixado. Então $\mathfrak{m}=\langle t\rangle$, e $\mathfrak{R}_{\Gamma, \xi}(\mathfrak{m})=k[t] / \mathfrak{m} \cong k$ se $\Gamma=\emptyset$ e $\mathfrak{R}_{\Gamma, \xi}(\mathfrak{m}) \cong k[d]$ se $\Gamma \neq \emptyset$. Então, no primeiro caso, existe um único módulo simples; enquanto no segundo, os módulos simples $S(\mathcal{O}, \Gamma, \xi, \mathfrak{N})$ são parametrizados por ideais maximais $\mathfrak{N} \subset k[d]$.

Para mais exemplos indicamos [BBF04].

\subsection{Tipo de representação e módulos indecomponíveis para $A_{n}, 1 \leq n \leq \infty$}

Basearemos esta seção em [BBF04][Seção 7].

Vamos classificar os blocos do tipo mansa na categoria dos módulos de peso localmente finitos sobre álgebras de Weyl $A_{n}$, finitas ou infinitas, sobre um corpo $k$. Também vamos determinar os módulos indecomponíveis nos blocos do tipo mansa. Estes resultados generalizam aqueles em [BB00] e [DGO96].

Vamos utilizar os seguintes conceitos que podem ser encontrados em [Dro80].

Definição 3.2.1. Seja $\mathbb{F}$ um corpo algebricamente fechado, e seja $\mathbb{F}\langle x, y\rangle$ uma álgebra associativa livre com unidade 1 sobre $\mathbb{F}$ em dois geradores $x$ e $y$. 
- Uma $\mathbb{F}$-categoria $\mathcal{C}$ é chamada de selvagem se existe um $\mathcal{C}$ - $\mathbb{F}\langle x, y\rangle$-bimódulo $M$, livre de posto finito como um $\mathbb{F}\langle x, y\rangle$-módulo à direita, tal que o funtor $M \otimes_{\mathbb{F}\langle x, y\rangle}-$ preserva indecomponibilidade e classes de isomorfismo.

- Uma $\mathbb{F}$-categoria $\mathcal{C}$ é chamada de mansa se, para cada vetor dimensão $\underline{d}$, existe uma localização $R=\mathbb{F}[x]_{f}$ com respeito à algum $f \in \mathbb{F}[x]$ e uma quantidade finita de $\mathcal{C}$ $R$-bimódulos $B_{1}, \ldots, B_{n}$ tal que cada $B_{j}$ é livre de posto finito como um $R$-módulo à direita, e tal que todo indecomponivel $X \in \mathcal{C}$-fdmod com $\operatorname{dim}_{\mathbb{F}} X(\alpha)=\underline{d}_{\alpha}$ é isomorfo a $B_{j} \otimes_{R} S$ para algum $j$ e algum $R$-módulo simples $S$.

No caso de um corpo arbitrário $\mathbb{F}$ dizemos que uma $\mathbb{F}$-categoria $\mathcal{C}$ é selvagem (resp. mansa) se ela é selvagem (resp. mansa) sobre o fecho algébrico $\overline{\mathbb{F}}$ de $\mathbb{F}$.

Vamos considerar que os $A_{n}$-módulos de peso $V$ que são localmente finitos, isto é, todos os $V_{\mathfrak{m}}$ são espaços vetoriais sobre $D / \mathfrak{m}$ com dimensão finita, em que $D=k\left[\tilde{t_{1}}, \ldots, \tilde{t_{n}}\right]$ e $\tilde{t}_{i}=\partial_{i} x_{i}$. Seja $\mathcal{W}^{l f}\left(A_{n}\right)$ a categoria dos $A_{n}$-módulos de peso localmente finitos. Para cada subconjunto $T \subseteq \mathfrak{m a x} D$ denotaremos por $\mathcal{W}_{T}^{l f}\left(A_{n}\right)$ a subcategoria plena em $\mathcal{W}^{l f}\left(A_{n}\right)$ consistindo de todos os módulos $V$ com $\operatorname{supp}(V) \subseteq T$. Os módulos de peso localmente finitos indecomponíveis sobre a álgebra de Weyl $A_{1}$ foram descritos em [DGO96] (veja também [BB00] para o caso $A_{n}(n<\infty)$ sobre um corpo algebricamente fechado). Por conta dos isomorfismos entre categorias das Subseções 3.1.2 e 3.1.3, podemos focar nas categorias $\mathcal{A}(\mathbb{F}, I)$ e $\mathcal{B}(\mathbb{F}, I, J, \tau)$ para um corpo $\mathbb{F}$ (que geralmente especificamos por $D / \mathfrak{m}$ ) e vamos considerar os módulos localmente finitos indecomponíveis sobre elas.

Segue de [BB00][Seção 2.6] que $\mathcal{B}(\mathbb{F}, I, J, \underline{\tau})$ é selvagem se $|I|+|J|>1$, e $\mathcal{A}(\mathbb{F}, I)$ é selvagem para $|I|>2$. Portanto, como o nosso objetivo é descrever os indecomponíveis nos blocos do tipo mansa, é suficiente descrever os indecomponíveis para as categorias $\mathcal{A}(\mathbb{F}, I)$ com $|I| \leq 2$ e para $\mathcal{B}(\mathbb{F}, I, J, \underline{\tau})$ com $|I|+|J|=1$ (que corresponde ao caso $n=1$ ).

Na Subseção 3.1.3 construímos os módulos simples para $\mathcal{A}(\mathbb{F}, I)$. A categoria $\mathcal{A}(\mathbb{F}, \emptyset)$ tem um único objeto $\omega$ e conjunto de morfismos $\mathbb{F} 1_{\omega}$. Então ela é uma álgebra simples, e qualquer módulo indecomponível de dimensão finita sobre $\mathcal{A}(\mathbb{F}, \emptyset)$ é simples.

\subsubsection{Indecomponíveis para $\mathcal{A}(\mathbb{F}, I),|I|=1$}

Lembremos que a categoria $\mathcal{A}(\mathbb{F}, I),|I|=1$, é isomorfa à categoria $\overline{\mathcal{Q}_{1}}=\mathbb{F} \mathcal{Q}_{1} / \mathcal{R}$ correspondendo ao seguinte quiver e relações:

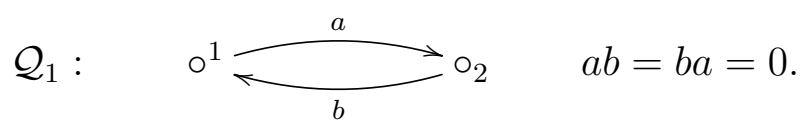

Vamos denotar por $S_{i}, i=1,2$, os módulos simples para $\overline{\mathcal{Q}_{1}}$. Logo são indecomponíveis. Agora seja $M_{a}$ (resp. $M_{b}$ ) o $\overline{\mathcal{Q}_{1}}$-módulo tal que $M_{a}(1)=M_{b}(1)=\mathbb{F} e_{1}, M_{a}(2)=M_{b}(2)=\mathbb{F} e_{2}$, em que a ação é dada por $a e_{1}:=e_{2}$ e $b e_{2}:=0$ (resp. $a e_{1}:=0$ e be $e_{2}:=e_{1}$ ). Dessas observações, segue a seguinte proposição:

Proposição 3.2.1. Os módulos $S_{1}, S_{2}, M_{a}, M_{b}$, constituem uma lista exaustiva de $\overline{\mathcal{Q}_{1}}$ módulos indecomponíveis de dimensão finita não isomorfos tomados dois a dois.

\subsubsection{Indecomponíveis para $\mathcal{A}(\mathbb{F}, I),|I|=2$}

Pode-se ver que a categoria $\mathcal{A}(\mathbb{F}, I),|I|=2$, é isomorfa a categoria $\overline{\mathcal{Q}_{2}}:=\mathbb{F} \mathcal{Q}_{2} / \mathcal{R}$ correspondendo ao seguinte quiver e relações: 


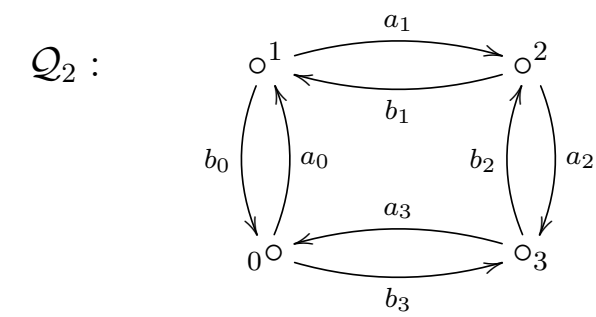

$$
a_{i} b_{i}=b_{i} a_{i}=0 \quad \text { para } i \in\{0,1,2,3\}
$$

$a_{i} a_{j}=b_{l} b_{m}$ para $i, j, l, m \in\{0,1,2,3\}$, sempre que for possível

Consideramos os objetos $0,1,2,3$ como os elementos de $\mathbb{Z}_{4}=\mathbb{Z} / 4 \mathbb{Z}$ e denotamos os correspondentes módulos simples por $S_{i}, i \in \mathbb{Z}_{4}$.

Agora, suponhamos que $M_{i}, i \in \mathbb{Z}_{4}$, é o $\overline{\mathcal{Q}_{2}}$-módulo tal que $M_{i}(j)=\mathbb{F} e_{j}$ para $j \in \mathbb{Z}_{4}$, em que a ação em $M_{i}$ é dada por $a_{i} e_{i}=e_{i+1}, a_{i+1} e_{i+1}=e_{i+2}, b_{i-1} e_{i}=e_{i-1}, b_{i-2} e_{i-1}=e_{i-2}$, e $u_{j} e_{k}=0$ para todas as outras instâncias de $u \in\{a, b\}$ e $j, k \in \mathbb{Z}_{4}$.

Para cada $n \in \mathbb{N}, n>1$, e $j \in \mathbb{Z}_{4}$ definimos o $\overline{\mathcal{Q}_{2}}$-módulo $M_{n, j, 0}$ (resp. $M_{n, j, 1}$ ) como segue. Consideremos $n$ elementos $e_{1}, \ldots, e_{n}$. Para $l \in \mathbb{Z}_{4}$, uma $\mathbb{F}$-base do espaço vetorial $M_{n, j, 0}(l)$ (resp. $\left.M_{n, j, 1}(l)\right)$ é o conjunto formado pelos $e_{k}$ tais que $j+k-1 \equiv l(\bmod 4)$. A ação de $a_{l}$ e $b_{l-1}$ em $M_{n, j, 0}(l)\left(\operatorname{resp} . M_{n, j, 1}(l)\right)$ é dada pelas regras:

$$
\begin{aligned}
& a_{l} e_{k}= \begin{cases}e_{k+1} & , \text { se } l \text { é par (resp. ímpar) }, k<n, \text { e } j+k-1 \equiv l(\bmod 4) ; \\
0 & , \text { caso contrário. }\end{cases} \\
& b_{l-1} e_{k}= \begin{cases}e_{k-1} & , \text { se } l \text { é par (resp. ímpar) }, k>1, \text { e } j+k-1 \equiv l(\bmod 4) ; \\
0 & , \text { caso contrário. }\end{cases}
\end{aligned}
$$

Em todos os outros casos, temos $u_{j} e_{k}=0$ para $u \in\{a, b\}$ e $j, k \in \mathbb{Z}_{4}$.

Denotaremos por $\operatorname{Irr}_{0} \mathbb{F}[x]$ o conjunto dos polinômios irredutíveis mônicos $f \neq x$ em $\mathbb{F}[x]$, e por $\operatorname{Ind}_{0} \mathbb{F}[x]=\left\{f^{n} \mid f \in \operatorname{Irr}_{0} \mathbb{F}[x]\right.$ e $\left.n \in \mathbb{N}\right\}$. Para cada $f(x)=x^{e}+\zeta_{e} x^{e-1}+\cdots+\zeta_{1}$ $\in \operatorname{Ind}_{0} \mathbb{F}[x]$, definimos o $\overline{\mathcal{Q}_{2}}$-módulo $M_{f, 1}$ (resp. $M_{f, 2}$ ) como segue: seja $M_{f, 1}(i):=\mathbb{F}^{e}$ (resp. $\left.M_{f, 2}(i):=\mathbb{F}^{e}\right)$ para $i \in \mathbb{Z}_{4}$, e definimos

$$
\begin{aligned}
M_{f, 1}\left(a_{0}\right) & =M_{f, 1}\left(a_{2}\right)=M_{f, 1}\left(b_{1}\right)=I_{e} \\
M_{f, 1}\left(b_{0}\right) & =M_{f, 1}\left(b_{2}\right)=M_{f, 1}\left(a_{1}\right)=M_{f, 1}\left(a_{3}\right)=0, \\
M_{f, 1}\left(b_{3}\right) & =\mathfrak{F}_{f}, \\
M_{f, 2}\left(b_{0}\right) & =M_{f, 2}\left(b_{2}\right)=M_{f, 2}\left(a_{1}\right)=I_{e}, \\
M_{f, 2}\left(a_{0}\right) & =M_{f, 2}\left(a_{2}\right)=M_{f, 2}\left(b_{1}\right)=M_{f, 2}\left(b_{3}\right)=0, \\
M_{f, 2}\left(a_{3}\right) & =\mathfrak{F}_{f},
\end{aligned}
$$

em que $\mathfrak{F}_{f}$ é a matriz companheira de Frobenius correspondente ao polinômio $f$ :

$$
\mathfrak{F}_{f}=\left(\begin{array}{ccccc}
0 & 0 & \cdots & 0 & -\zeta_{1} \\
1 & 0 & \cdots & 0 & -\zeta_{2} \\
0 & 1 & \cdots & 0 & \vdots \\
\vdots & \vdots & \ddots & \vdots & -\zeta_{e-1} \\
0 & 0 & \cdots & 1 & -\zeta_{e}
\end{array}\right)
$$


Como consequência da Proposição 3.3.1 em [BB00] temos:

Proposição 3.2.2. Os módulos $S_{i}, M_{i}, M_{n, i, 0}, M_{n, i, 1}, M_{f, 1}, M_{f, 2}$, em que $f \in \operatorname{Ind}_{0} \mathbb{F}[x]$, $i$ $\in \mathbb{Z}_{4}$, e $n \in \mathbb{N}, n>1$, constituem uma lista exaustiva de $\overline{\mathcal{Q}_{2}}$-módulos indecomponíveis de dimensão finita não isomorfos tomados dois a dois.

Teorema 3.2.1. Seja $\mathcal{O}$ uma órbita de $\mathcal{G}$ em max $D$ para a álgebra de Weyl $A=A_{n}(1 \leq$ $n \leq \infty)$ sobre $k$ (assume-se que $\mathcal{O}$ possui break maximal quando $\mathcal{O}$ é degenerada e $n=\infty$ ).

1. Se $\operatorname{char}(k)=0$, então a categoria $\mathcal{W}_{\mathcal{O}}^{l f}(A)$ é mansa se, e somente se, ou $\mathcal{O}$ é não degenerada ou a ordem do break maximal é menor ou igual à 2;

2. Se $\operatorname{char}(k)=p>0$, então a categoria $\mathcal{W}_{\mathcal{O}}^{l f}(A)$ é mansa se, e somente se, $n=1$.

Demonstração. 1. $(\operatorname{char}(k)=0)$ Se $\mathcal{O}$ é não degenerada com ideal maximal fixado $\mathfrak{m}$, então $\mathcal{W}_{\mathcal{O}}^{l f}(A) \approx \mathcal{A}(D / \mathfrak{m}, \emptyset)$-fdmod $\approx(D / \mathfrak{m})$-fdmod pelas Proposições 3.1 .1 e 3.1.2, então $\mathcal{W}_{\mathcal{O}}^{l f}(A)$ é mansa. Se $\mathcal{O}$ é degenerada com break maximal designado $\mathfrak{m}$ com ordem $|\underline{I}|=1$, então $\mathcal{W}_{\mathcal{O}}^{l f}(A) \approx \mathcal{A}(D / \mathfrak{m}, \underline{I})$-fdmod pelas Proposições 3.1.1 e 3.1.2, portanto $\mathcal{W}_{\mathcal{O}}^{l f}(A)$ é mansa pela Proposição 3.2.1. Se $\mathcal{O}$ é degenerada com break maximal $\mathfrak{m}$ de ordem 2, então $\mathcal{W}_{\mathcal{O}}^{l f}(A) \approx \mathcal{A}(D / \mathfrak{m}, \underline{I})$-fdmod, $|\underline{I}|=2$, pelas Proposições 3.1.1 e 3.1.2, portanto $\mathcal{W}_{\mathcal{O}}^{l f}(A)$ é mansa pela Proposição 3.2.2.

Reciprocamente, a afirmação segue do fato que $\mathcal{A}(\mathbb{F}, I)$ é selvagem para $|I|>2$.

2. $(\operatorname{char}(k)=p>0)$ Se $\mathcal{B}(\mathbb{F}, I, J, \underline{\tau})$ é mansa, então $|I|+|J|=1$ (pois $\mathcal{B}(\mathbb{F}, I, J, \underline{\tau})$ é selvagem para $|I|+|J|>1$ ). Isto corresponde ao caso $n=1$, o qual foi tratado em [DGO96] e [BB00][Seção 4.1].

A recíproca é consequência do Teorema 5.7 em [DGO96].

\subsubsection{Indecomponíveis para blocos do tipo mansa}

Pelo Teorema 3.2.1(2), $A_{1}$ é a única álgebra de Weyl de característica $p$ que possui blocos do tipo mansa, e os módulos indecomponíveis nestes blocos foram determinados em [BB00][Seção 4.1]. Então, o que segue será concentrado no caso de característica 0 e na construção dos $A_{n}$-módulos de peso indecomponíveis para os blocos do tipo mansa, usando as Proposições 3.1.1 e 3.1.2, e a descrição dos $\mathcal{A}(\mathbb{F}, I)$-módulos no caso $|I| \leq 2$ (por conta do Teorema 3.2.1(1)).

Definição 3.2.2. Vamos assumir que char $(k)=0$, e que a órbita $\mathcal{O}$ tem break maximal $\mathfrak{m}$ de ordem 1 com respeito a $i$.

- $M(\mathcal{O}, \mathfrak{p}):=S(\mathcal{O}, \mathfrak{p})$ para $\mathfrak{p} \in \mathfrak{B}_{\mathcal{O}}($ cf. Subseção 3.1.3);

- $M\left(\mathcal{O}, x_{i}\right):=\bigoplus_{\mathfrak{n} \in \mathcal{O}} D / \mathfrak{n}$, tal que para cada $j$ :

$$
x_{j}(d+\mathfrak{n}):=\sigma_{j}(d)+\sigma_{j}(\mathfrak{n})
$$

$e$

$$
\partial_{j}(d+\mathfrak{n}):= \begin{cases}0 & , \text { se } j=i, \sigma_{i}^{-1}(\mathfrak{n}) \in \mathcal{O}_{\mathfrak{m}}, \text { e } \mathfrak{n} \in \mathcal{O}_{\sigma_{i}(\mathfrak{m})} \\ \tilde{t_{j}} \sigma_{j}^{-1}(d)+\sigma_{j}^{-1}(\mathfrak{n}) & , \text { caso contrário. }\end{cases}
$$


- $M\left(\mathcal{O}, \partial_{i}\right):=\bigoplus_{\mathfrak{n} \in \mathcal{O}} D / \mathfrak{n}$, tal que para $j=1, \ldots, n$ :

$$
x_{j}(d+\mathfrak{n}):= \begin{cases}0 & , \text { se } j=i, \mathfrak{n} \in \mathcal{O}_{\mathfrak{m}}, \text { e } \sigma_{i}(\mathfrak{n}) \in \mathcal{O}_{\sigma_{i}(\mathfrak{m})} ; \\ \sigma_{j}(d)+\sigma_{j}(\mathfrak{n}) & , \text { caso contrário. }\end{cases}
$$

$e$

$$
\partial_{j}(d+\mathfrak{n}):=\tilde{t_{j}} \sigma_{i}^{-1}(d)+\sigma_{j}^{-1}(\mathfrak{n}) .
$$

Observação 3.2.1. Sejam $\mathcal{O}, \mathfrak{m}, i$ como na Definição 3.2.2. Então:

- $M(\mathcal{O}, \mathfrak{m}) \cong A / A\left(\mathfrak{m}, x_{i}\right)$;

- $M\left(\mathcal{O}, \sigma_{i}(\mathfrak{m})\right) \cong A / A\left(\sigma_{i}(\mathfrak{m}), \partial_{i}\right)$;

- $M\left(\mathcal{O}, x_{i}\right) \cong A / A \mathfrak{m}$;

- $M\left(\mathcal{O}, \partial_{i}\right) \cong A / A \sigma_{i}(\mathfrak{m})$.

Agora vamos supor que $\mathcal{O}$ é uma órbita de $D=k\left[\tilde{t_{1}}, \ldots, \tilde{t_{n}}\right]$ sob o grupo de automorfismos $\mathcal{G}$ de $A_{n}$ com break maximal $\mathfrak{m}$ com respeito a $i, j \in\{1, \ldots, n\}(i \neq j)$. Assumindo $\mathbb{F}=$ $D / \mathfrak{m}$, definimos a aplicação $\gamma: \mathfrak{B}_{\mathcal{O}} \rightarrow \mathbb{Z}_{4}$ pela seguinte regra: $\gamma(\mathfrak{m})=0, \gamma\left(\sigma_{i}(\mathfrak{m})\right)=3$, $\gamma\left(\sigma_{j}(\mathfrak{m})\right)=1$ e $\gamma\left(\sigma_{i} \sigma_{j}(\mathfrak{m})\right)=2$.

Para $M$ um $\overline{\mathcal{Q}_{2}}$-módulo, associamos o correspondente módulo de peso $\mathfrak{M}$ sobre $A=A_{n}$ de acordo com o seguinte procedimento: para $\mathfrak{p} \in \mathfrak{B}_{\mathcal{O}}$ suponhamos $\mathfrak{M}(\mathfrak{p}) \cong M(\gamma(\mathfrak{p}))$ (como $D / \mathfrak{m}$-espaços vetoriais); via a aplicação que envia $v \in M(\gamma(\mathfrak{p}))$ em $v_{\mathfrak{p}} \in \mathfrak{M}(\mathfrak{p})$. Para cada $\mathfrak{n}$ $\in \mathcal{O}_{\mathfrak{p}}$, suponhamos $\mathfrak{M}(\mathfrak{n}) \cong \mathfrak{M}(\mathfrak{p})$ via $v_{\mathfrak{p}} \mapsto v_{\mathfrak{n}}$ para todo $v \in M(\gamma(\mathfrak{p}))$.

Como $\sigma_{\mathfrak{n}}^{-1}$ induz um isomorfismo de $D / \mathfrak{n}$ em $D / \mathfrak{m}$, podemos considerar $\mathfrak{M}(\mathfrak{n})$ como um $D / \mathfrak{n}$-módulo; então como um $D$-módulo que é aniquilado por $\mathfrak{n}$. Portanto $\mathfrak{M}=\bigoplus_{\mathfrak{n} \in \mathcal{O}} \mathfrak{M}(\mathfrak{n})$ em que $\mathfrak{M}(\mathfrak{n})=\{u \in \mathfrak{M} \mid \mathfrak{n} u=\{0\}\}$. Para a $A_{n}$-ação definimos:

- Se $\mathfrak{n} \in \mathcal{O}_{\mathfrak{m}}$ e $\sigma_{i}(\mathfrak{n}) \in \mathcal{O}_{\sigma_{i}(\mathfrak{m})}$ :

$$
\begin{gathered}
x_{i} v_{\mathfrak{n}}=\left(M\left(b_{3}\right) v\right)_{\sigma_{i}(\mathfrak{n})} \quad, \quad \partial_{i} v_{\sigma_{i}(\mathfrak{n})}=\left(M\left(a_{3}\right) v\right)_{\mathfrak{n}} ; \\
x_{i} v_{\sigma_{j}(\mathfrak{n})}=\left(M\left(a_{1}\right) v\right)_{\sigma_{i} \sigma_{j}(\mathfrak{n})} \quad, \quad \partial_{i} v_{\sigma_{i} \sigma_{j}(\mathfrak{n})}=\left(M\left(b_{1}\right) v\right)_{\sigma_{j}(\mathfrak{n})} ;
\end{gathered}
$$

- Se $\mathfrak{n} \in \mathcal{O}_{\mathfrak{m}}$ e $\sigma_{j}(\mathfrak{n}) \in \mathcal{O}_{\sigma_{j}(\mathfrak{m})}$ :

$$
\begin{array}{cl}
x_{j} v_{\mathfrak{n}}=\left(M\left(a_{0}\right) v\right)_{\sigma_{j}(\mathfrak{n})} \quad, \quad \partial_{j} v_{\sigma_{j}(\mathfrak{n})}=\left(M\left(b_{0}\right) v\right)_{\mathfrak{n}} ; \\
x_{j} v_{\sigma_{i}(\mathfrak{n})}=\left(M\left(b_{2}\right) v\right)_{\sigma_{i} \sigma_{j}(\mathfrak{n})} \quad, \quad \partial_{j} v_{\sigma_{i} \sigma_{j}(\mathfrak{n})}=\left(M\left(a_{2}\right) v\right)_{\sigma_{i}(\mathfrak{n})} ;
\end{array}
$$

- Em todos os outros casos:

$$
x_{l} v_{\mathfrak{n}}=v_{\sigma_{l}(\mathfrak{n})} \quad, \quad \partial_{l} v_{\mathfrak{n}}=\tilde{t}_{l} v_{\sigma_{l}^{-1}(\mathfrak{n})} .
$$

Para $\mathfrak{p} \in \mathfrak{B}_{\mathcal{O}}$, denotamos por $S(\mathcal{O}, \mathfrak{p}), M(\mathcal{O}, \mathfrak{p}), M(\mathcal{O}, n, \mathfrak{p}, 0), M(\mathcal{O}, n, \mathfrak{p}, 1), M(\mathcal{O}, f, 1)$, e $M(\mathcal{O}, f, 2)$ os $A_{n}$-módulos de peso que correspondem via este processo aos $\overline{\mathcal{Q}_{2}}$-módulos $S_{\gamma(\mathfrak{p})}, M_{\gamma(\mathfrak{p})}, M_{n, \gamma(\mathfrak{p}), 0}, M_{n, \gamma(\mathfrak{p}), 1}, M_{f, 1}$ e $M_{f, 2}$ respectivamente como na Proposição 3.2.2.

Observação 3.2.2. Sejam $\mathcal{O}, \mathfrak{m}, i, j$, e $\mathfrak{p} \in \mathfrak{B}_{\mathcal{O}}$ como descrito acima. Então:

- $S(\mathcal{O}, \mathfrak{m}) \cong A / A\left(\mathfrak{m}, x_{i}, x_{j}\right)$; 
- $S\left(\mathcal{O}, \sigma_{i}(\mathfrak{m})\right) \cong A / A\left(\sigma_{i}(\mathfrak{m}), \partial_{i}, x_{j}\right)$

- $S\left(\mathcal{O}, \sigma_{j}(\mathfrak{m})\right) \cong A / A\left(\sigma_{j}(\mathfrak{m}), \partial_{j}, x_{i}\right)$

- $S\left(\mathcal{O}, \sigma_{i} \sigma_{j}(\mathfrak{m})\right) \cong A / A\left(\sigma_{i} \sigma_{j}(\mathfrak{m}), \partial_{i}, \partial_{j}\right)$;

- $M(\mathcal{O}, \mathfrak{p}) \cong A / A \mathfrak{p}$

Teorema 3.2.2. Seja $\mathcal{O}$ uma órbita de $\mathcal{G}$ em max D para a álgebra de Weyl $A_{n}(1 \leq n \leq \infty)$ sobre um corpo $k$ de característica 0 (assume-se que $\mathcal{O}$ tem break maximal se $\mathcal{O}$ é degenerada $e n=\infty)$.

- $S e \mathcal{O}$ é não degenerada, então o módulo simples $S(\mathcal{O})$, é o único (a menos de isomorfismo) módulo indecomponível em $\mathcal{W}_{\mathcal{O}}^{l f}\left(A_{n}\right)$;

- $S e \mathcal{O}$ tem um break maximal $\mathfrak{m}$ de ordem 1 com respeito a $i$, então os módulos $S(\mathcal{O}, \mathfrak{m})$, $S\left(\mathcal{O}, \sigma_{i}(\mathfrak{m})\right), M\left(\mathcal{O}, x_{i}\right)$ e $M\left(\mathcal{O}, \partial_{i}\right)$, constituem uma lista exaustiva de módulos indecomponíveis não isomorfos tomados dois a dois em $\mathcal{W}_{\mathcal{O}}^{l f}\left(A_{n}\right)$;

- Se $\mathcal{O}$ tem um break maximal $\mathfrak{m}$ de ordem 2 com respeito a $i$ e $j$, então os módulos $S(\mathcal{O}, \mathfrak{p}), M(\mathcal{O}, \mathfrak{p}), M(\mathcal{O}, n, \mathfrak{p}, 0), M(\mathcal{O}, n, \mathfrak{p}, 1), M(\mathcal{O}, f, 1)$, e $M(\mathcal{O}, f, 2)$, em que $f \in$ Ind $_{0}(D / \mathfrak{m})[x], \mathfrak{p} \in \mathfrak{B}_{\mathcal{O}}$ e $n \in \mathbb{N}, n>1$, constituem uma lista exaustiva de módulos indecomponíveis não isomorfos tomados dois a dois em $\mathcal{W}_{\mathcal{O}}^{l f}\left(A_{n}\right)$.

Demonstração. Como na demonstração do Teorema 3.1.1, usaremos o funtor $F^{\prime} E G$, em que $E$ é um funtor equivalência $\mathcal{C}_{\mathcal{O}} \otimes_{\mathcal{S}_{\mathcal{O}}}-, F^{\prime}$ é dado na demonstração da Proposição 3.1.1, e $G$ é definido na demonstração da Proposição 3.1.2.

1. Se a órbita $\mathcal{O}$ é não degenerada, então segue das Proposições 3.1.1 e 3.1.2, que $\mathcal{W}_{\mathcal{O}}^{l f}\left(A_{n}\right)$ $\approx \mathcal{A}(D / \mathfrak{m}, \emptyset)$-fdmod $\approx D / \mathfrak{m}$-fdmod. Portanto todo módulo indecomponível é simples nesse caso, e a afirmação segue do Teorema 3.2.1.

2. Vamos assumir que $\mathcal{O}$ tem um break maximal $\mathfrak{m}$ de ordem 1 com respeito a $i$. Então:

$$
\begin{aligned}
& F^{\prime} E G\left(S_{1}\right) \cong S(\mathcal{O}, \mathfrak{m}) \quad, \quad F^{\prime} E G\left(S_{2}\right) \cong S\left(\mathcal{O}, \sigma_{i}(\mathfrak{m})\right), \\
& F^{\prime} E G\left(M_{a}\right) \cong M\left(\mathcal{O}, x_{i}\right) \quad, \quad F^{\prime} E G\left(M_{b}\right) \cong M\left(\mathcal{O}, \partial_{i}\right) .
\end{aligned}
$$

Consequentemente, o resultado neste caso segue das Proposições 3.1.1, 3.1.2 e 3.2.1.

3. Suponhamos que $\mathcal{O}$ tem um break maximal $\mathfrak{m}$ de ordem 2 com respeito a $i$ e $j$. Seja $\gamma: \overline{\mathcal{Q}_{2}} \rightarrow \mathfrak{B}_{\mathcal{O}}$ como definido anteriormente. Assim:

$$
\begin{gathered}
F^{\prime} E G\left(S_{k}\right) \cong S\left(\mathcal{O}, \gamma^{-1}(k)\right) \quad, \quad F^{\prime} E G\left(M_{k}\right) \cong M\left(\mathcal{O}, \gamma^{-1}(k)\right), \\
F^{\prime} E G\left(M_{n, k, l}\right) \cong M\left(\mathcal{O}, n, \gamma^{-1}(k), l\right) \quad, \quad F^{\prime} E G\left(M_{f, s}\right) \cong M_{\mathcal{O}, f, s},
\end{gathered}
$$

em que $k \in \mathbb{Z}_{4}, l \in\{0,1\}$, e $s \in\{1,2\}$, obtemos o resultado desejado usando as Proposições 3.1.1, 3.1.2 e 3.2.2. 


\subsection{Módulos de peso simples sobre $\boldsymbol{A}=\boldsymbol{A}_{k, \mathrm{I}}$}

A classificação dos $A_{\infty}$-módulos de peso simples dada por Bekkert, Benkart e Futorny em [BBF04] foi parcial. Contudo, em [FGM14] existe uma classificação completa dos módulos de peso simples sobre álgebras de Weyl de dimensão infinita $A=A_{k, \mathrm{I}}$.

Basearemos essa seção em [FGM14][3.5].

Considere o conjunto:

$$
S:=\left\{\mathbf{m} \in \mathbf{p}+\mathbb{Z}_{f}^{\mathrm{I}}: \forall i, p_{i} \in\{0,1,2, \ldots\} \Rightarrow m_{i} \in\{0,1,2, \ldots\}\right\}
$$

e seja $N:=\bigoplus_{\mathbf{m} \in S} B(\mathbf{p})_{\mathbf{m}}$. Então $N$ é um submódulo de $B(\mathbf{p})$. De fato, $N \subseteq B(\mathbf{p})$, e considerando um elemento qualquer $n=n_{1} \oplus \ldots \oplus n_{s}$ de $N$, em que $n_{j} \in B(\mathbf{p})_{\mathbf{v}_{j}}$. Para $i$ $\in \mathrm{I}, X_{i} n_{j} \in B(\mathbf{p})_{\mathbf{v}_{j}+\mathbf{e}_{i}}$ e $Y_{i} n_{j} \in B(\mathbf{p})_{\mathbf{v}_{j}-\mathbf{e}_{i}}$, em que $\mathbf{e}_{i}$ tem 1 na $i$-ésima coordenada e 0 nas outras. Então para qualquer $a \in A$, $a n_{j} \in B(\mathbf{p})_{\mathbf{v}_{j} \pm \mathbf{u}_{j}}$, em que $\mathbf{u}_{j} \in \mathbb{Z}_{f}^{\mathrm{I}}$. Logo an $\in B(\mathbf{p})_{\mathbf{v}_{1} \pm \mathbf{u}_{1}} \bigoplus \ldots \oplus B(\mathbf{p})_{\mathbf{v}_{s} \pm \mathbf{u}_{s}}$. Como cada $\mathbf{v}_{s} \pm \mathbf{u}_{s}=\mathbf{v}_{s}$ em $\mathbf{p}+\mathbb{Z}_{f}^{\mathrm{I}}$, temos que an $\in$ $B(\mathbf{p})_{\mathbf{v}_{1}} \bigoplus \ldots \bigoplus B(\mathbf{p})_{\mathbf{v}_{s}}$, portanto, an $\in N$.

Considere o conjunto:

$$
S^{\prime}:=\left\{\mathbf{m} \in S: \forall i, p_{i} \in\{-1,-2, \ldots\} \Rightarrow m_{i} \in\{0,1,2, \ldots\}\right\}
$$

e seja $N^{\prime}:=\bigoplus_{\mathbf{m} \in S^{\prime}} B(\mathbf{p})_{\mathbf{m}} \subseteq N$. Então $N^{\prime}$ é um submódulo de $N$, por um argumento semelhante ao dado no parágrafo anterior.

Observamos que os elementos de $S^{\prime}$ são todos os elementos $\mathbf{m}$ da classe $\mathbf{p}+\mathbb{Z}_{f}^{\mathrm{I}}$, em que $p_{i} \in \mathbb{Z}$ implica que $m_{i} \in \mathbb{N}$, para todo $i$.

Para $\mathbf{p} \in k^{\mathrm{I}}$ denotemos por $\overline{\mathbf{p}}$ o conjunto de todo $\mathbf{k} \in \mathbf{p}+\mathbb{Z}_{f}^{\mathrm{I}}$ que satisfaz as seguintes condições para todo $i \in \mathrm{I}$ :

$$
\begin{gathered}
p_{i} \in\{-1,-2,-3, \ldots\} \Longrightarrow k_{i} \in\{-1,-2,-3, \ldots\} \\
p_{i} \in\{0,1,2,3, \ldots\} \Longrightarrow k_{i} \in\{0,1,2,3, \ldots\}
\end{gathered}
$$

Seja $\mathbf{m} \in S \backslash S^{\prime}$. Logo, se $p_{i} \in\{0,1,2,3, \ldots\}$ então $m_{i} \in\{0,1,2,3, \ldots\}$ e se $p_{i} \in$ $\{-1,-2,-3, \ldots\}$ então $m_{i} \notin\{0,1,2,3 \ldots\}$. Mas $\mathbf{m}=\mathbf{p}+\mathbf{v}$, para algum $\mathbf{v} \in \mathbb{Z}_{f}^{\mathrm{I}}$. Então se $p_{i} \in\{-1,-2,-3, \ldots\}, m_{i}=p_{i}+v_{i}$ deve pertencer a $\mathbb{Z} \backslash\{0,1,2,3 \ldots\}$. Então podemos concluir que, se $p_{i} \in\{-1,-2,-3, \ldots\}$, então $m_{i} \in\{-1,-2,-3, \ldots\}$.

Temos que $S \backslash S^{\prime}=\operatorname{supp}\left(N / N^{\prime}\right)=\overline{\mathbf{p}}$.

Proposição 3.3.1. Para $\mathbf{p} \in k^{\mathrm{I}}$ temos que:

1. $\operatorname{supp}(L(\mathbf{p}))=\overline{\mathbf{p}}$;

2. Se $\mathbf{s} \in k^{\mathrm{I}}$, entãa $L(\mathbf{p}) \cong L(\mathbf{s})$ se, e somente se, $\mathbf{s} \in \overline{\mathbf{p}}$.

Demonstração. Primeiramente vamos mostrar que o módulo $N / N^{\prime}$ é simples. Seja $\mathbf{m} \in \overline{\mathbf{p}}$ e seja $v \in\left(N / N^{\prime}\right)_{\mathbf{m}}$ um elemento não nulo. Temos que mostrar que $v$ gera $N / N^{\prime}$. Sabemos que um $R$-módulo $M$ é simples se, e somente se, dados quaisquer $u, v \in M$ não nulos, existe $a \in$ $R$ tal que $a u=v$. Então é suficiente verificar que, dado $i \in \mathrm{I}$, temos $X_{i} v \neq 0$ se $m_{i} \neq-1 \mathrm{e}$ temos $Y_{i} v \neq 0$ se $m_{i} \neq 0$. Entretanto, ambas afirmações seguem diretamente das definições. Portanto $N / N^{\prime}$ é um $A$-módulo simples.

Como $\left(N / N^{\prime}\right)_{\mathbf{p}} \neq\{0\}$, por construção, temos que $N / N^{\prime} \cong L(\mathbf{p})$, pela Proposição 2.5.5(2). Agora 1. segue por construção e 2. segue por 1. e pela Proposição 2.5.5. De fato, se $L(\mathbf{p}) \cong$ 
$L(\mathbf{s})$, pelo item 1., $\operatorname{supp}(L(\mathbf{p}))=\overline{\mathbf{p}}=\operatorname{supp}(L(\mathbf{s}))=\overline{\mathbf{s}}, \log$ o $\mathbf{s} \in \overline{\mathbf{p}}$. Reciprocamente, se $\mathbf{s} \in \overline{\mathbf{p}}$, então $L(\mathbf{p})$ é simples e $L(\mathbf{p})_{\mathbf{s}} \neq\{0\}$; mas pela Proposição 2.5.5(2), $L(\mathbf{s})$ é o único $A$-módulo simples, a menos de isomorfismo, com essa propriedade, portanto $L(\mathbf{p}) \cong L(\mathbf{s})$.

Os resultados descritos acima podem ser resumidos como segue: seja $\sim$ uma relação de equivalência em $k^{\mathrm{I}}$ definida como segue: $\mathbf{p} \sim \mathbf{m}$ se, e somente se, $\overline{\mathbf{p}}=\overline{\mathbf{m}}$. Claramente a relação descrita é reflexiva e simétrica. Além disso, se $\mathbf{p} \sim \mathbf{q}$ e $\mathbf{q} \sim \mathbf{m}$, ou seja, $\overline{\mathbf{p}}=\overline{\mathbf{q}}$ e $\overline{\mathbf{q}}=\overline{\mathbf{m}}$, então $\overline{\mathbf{p}}=\overline{\mathbf{m}}$, logo $\mathbf{p} \sim \mathbf{m}$. Portanto a relação também é transitiva.

Teorema 3.3.1 (Teorema 5 de [FGM14] - Classificação dos $A$-módulos de peso simples). $A$ aplicação $\overline{\mathbf{p}} \mapsto L(\mathbf{p}), \mathbf{p} \in k^{\mathrm{I}}$, é uma bijeção entre $k^{\mathrm{I}} / \sim$ e o conjunto das classes de isomorfismos dos A-módulos de peso simples.

Abaixo segue um exemplo da não existência de break maximais quando tomamos módulos de peso sobre $A_{n}$, $\operatorname{com} n=\infty$ :

Exemplo 3.3.1. Tome $\mathbf{p}=(1,1, \ldots)$, isto é, $p_{i}=1$, para todo $i$. O módulo simples $L(\mathbf{p})$ é localmente finito com respeito à cada $\partial_{i}$, entretanto, não existe $v \in L(\mathbf{p})$ para o qual $\partial_{i} v=0$, para todo $i$. Exemplos similares são encontrados em [MZ11][Subseção 4.2].

Observação 3.3.1. O Teorema 3.3.1 pode ser transferido mutatis mutandis para o produto tensorial de álgebras de Weyl generalizadas de posto um no sentido de [Bav92a] associadas $\grave{a} k[x]$ e $\sigma: k[x] \rightarrow k[x]$, definido por $\sigma(x)=x+1$.

\subsection{Realizações via polinômios torcidos}

Basearemos esta seção em [FGM14][Seção 3.6].

Vamos definir um anel de polinômios torcido e mais detalhes podem ser encontrados em [GJ89][Cap. 1].

Definição 3.4.1. Seja $\alpha$ um endomorfismo de um anel $R$. Uma $\alpha$-derivação à esquerda de $R$ é qualquer aplicação aditiva $\delta: R \rightarrow R$ tal que $\delta(r s)=\alpha(r) \delta(s)+\delta(r) s$ para todos $r, s \in$ $R$. Por outro lado, uma $\alpha$-derivação à direita de $R$ é qualquer aplicação aditiva $\delta: R \rightarrow R$ tal $q u e \delta(r s)=\delta(r) \alpha(s)+r \delta(s)$ para todos $r, s \in R$. Se $\alpha$ é a identidade em $R$, uma $\alpha$-derivação de $R$ é simplesmente uma derivação de $R$.

Temos que $\delta(1)=\delta(1 \cdot 1)=\alpha(1) \delta(1)+\delta(1) \cdot 1=2 \delta(1), \operatorname{logo} \delta(1)=0$.

Por exemplo, em qualquer anel de polinômios $k[x]$ a derivada usual $d / d x$ é uma derivação. Dado qualquer endomorfismo $\alpha$ de um anel $R$, e qualquer elemento $d \in R$, a regra $\delta(r)=d r-$ $\alpha(r) d$ define uma $\alpha$-derivação de $R$. De fato, $\delta(r s)=d(r s)-\alpha(r s) d=(d r) s-\alpha(r) \alpha(s) d=$ $(\delta(r)+\alpha(r) d) s-\alpha(r) \alpha(s) d=\delta(r) s+\alpha(r)(d s-\alpha(s) d), \log 0 \delta(r s)=\delta(r) s+\alpha(r) \delta(s)$.

Dados um anel $R$, um endomorfismo $\alpha$ de $R$, e uma $\alpha$-derivação $\delta$ de $R$, vamos construir um anel de polinômios torcido $S$. Ele será um $R$-módulo à esquerda livre, com base $1, \theta, \theta^{2}, \ldots$. Podemos obter o anel de polinômios torcido $S$ como um subanel do anel de endomorfismos de um grupo abeliano adequado. Assim $S$ vai satisfazer todos os axiomas de um anel.

Proposição 3.4.1. Sejam $R$ um anel, $\alpha$ um endomorfismo de $R$ e $\delta$ uma $\alpha$-derivação de $R$. Então existe um anel $S$, contendo $R$ como um subanel, tal que $S$ é um $R$-módulo à esquerda livre com uma base da forma $1, \theta, \theta^{2}, \ldots, e \theta r=\alpha(r) \theta+\delta(r)$, para todo $r \in R$. 
Demonstração. Seja $E=\operatorname{End}_{\mathbb{Z}}(R[x])$, em que $x$ é uma indeterminada. Existe um homomorfismo de anéis injetivo $R \rightarrow E$, pois qualquer elemento $r \in R$ corresponde a multiplicação à esquerda por $r$ em $R[x]$. Nós identificamos $R$ com sua imagem sob este homomorfismo, então $R$ é agora um subanel de $E$.

Vamos estender $\alpha$ e $\delta$ à homomorfismos em $E$ em que $\alpha\left(r x^{i}\right)=\alpha(r) x^{i}$ e $\delta\left(r x^{i}\right)=\delta(r) x^{i}$, para todo $r \in R$ e $i=0,1,2, \ldots$.. Então $\alpha$ se transforma em um endomorfismo de $R[x]$ e $\delta$ uma $\alpha$-derivação de $R[x]$. Agora defina $\theta \in E$ de acordo com a regra: $\theta(f)=\alpha(f) x+\delta(f)$. Para $r \in R$ e $f \in R[x]$ temos que:

$$
(\theta r)(f)=\alpha(r f) x+\delta(r f)=\alpha(r) \alpha(f) x+\alpha(r) \delta(f)+\delta(r) f=\alpha(r) \theta(f)+\delta(r) f
$$

Então $\theta r=\alpha(r) \theta+\delta(r)$, para todo $r \in R$. Em particular $\theta R \subseteq R \theta+R$.

Da relação $\theta R \subseteq R \theta+R$, segue por indução que $\theta^{i} R \subseteq R \theta^{i}+R \theta^{i-1}+\cdots+R \theta+R$, para todo $i=0,1,2, \ldots$. Consequentemente, $\left(R \theta^{i}\right)\left(R \theta^{j}\right)=R\left(\theta^{i} R\right) \theta^{j} \subseteq R \theta^{i+j}+R \theta^{i+j-1}+$ $\cdots+R \theta^{j+1}+R \theta^{j}$, para todos $i, j=0,1,2, \ldots$. Portanto o conjunto $\sum R \theta^{i}$ é fechado sob multiplicação. Então $\sum R \theta^{i}$ é um subanel de $E$, que vamos denotar por $S$. Então $S$ é gerado como $R$-módulo à esquerda por $1, \theta, \theta^{2}, \ldots$.

Resta mostrar que $1, \theta, \theta^{2}, \ldots$ é um conjunto linearmente independente à esquerda sobre $R$. Observamos que $\theta\left(x^{j}\right)=\alpha\left(x^{j}\right) x+\delta\left(x^{j}\right)=\alpha(1) x^{j} x+\delta(1) x^{j}=x^{j+1}$ para todo $j=0,1, \ldots$ e $\theta^{i}\left(x^{0}\right)=x^{i}$ para todo $i=0,1, \ldots$. Dado um elemento $s=r_{0}+r_{1} \theta+\cdots+r_{n} \theta^{n} \in S$, com $r_{0}, \ldots, r_{n} \in R$, segue que $s=s\left(x^{0}\right)=r_{0}+r_{1} x+\cdots+r_{n} x^{n}$, e então $s=0$ se, e somente se, $r_{0}=\cdots=r_{n}=0$. Então os $\theta^{i}$ s são linearmente independentes à esquerda sobre $R$, e portanto formam uma base para $S$ como $R$-módulo à esquerda.

O anel $S$ da Proposição 3.4.1 é essencialmente único no seguinte sentido: sejam $S_{1}$ e $S_{2}$ extensões de $R$ tais que $S_{i}$ é $R$-módulo à esquerda livre com base na forma $1, \theta_{i}, \theta_{i}^{2}, \ldots$, e $\theta_{i} r=\alpha(r) \theta_{i}+\delta(r)$, para todo $r \in R$ :

1. Se $\phi: R \rightarrow T$ é um homomorfismo de anéis e existe um elemento $\xi \in T$ tal que $\xi \phi(r)=\phi \alpha(r) \xi+\phi \delta(r)$, para todo $r \in R$, então $\phi$ se estende unicamente para um homomorfismo de anéis $\Phi: S_{1} \rightarrow T$ tal que $\Phi\left(\theta_{1}\right)=\xi$.

2. O homomorfismo identidade em $R$ se estende a um isomorfismo de anéis $\Phi: S_{1} \rightarrow S_{2}$ tal que $\Phi\left(\theta_{1}\right)=\theta_{2}$.

Definição 3.4.2. Sejam $R$ um anel, $\alpha$ um endomorfismo de $R$, e $\delta$ uma $\alpha$-derivação de $R$. $O$ anel $S$ construído na Proposiçãa 3.4.1, é denotado por $R[\theta ; \alpha, \delta]$ e é chamado de anel polinomial torcido ou extensão de Ore de $R$. No caso que $\alpha$ é a identidade em $R$, abreviaremos $R[\theta ; \alpha, \delta]$ por $R[\theta ; \delta]$, e chamaremos este anel de anel de operador diferencial (formal). No caso $\delta=0$, abreviaremos $R[\theta ; \alpha, \delta]$ por $R[\theta ; \alpha]$

Sejam $R$ um anel, um endomorfismo $\alpha$ de $R$, e uma $\alpha$-derivação à direita $\delta$ em $R$. O correspondente anel de polinômios torcido é um $R$-módulo à direita livre com uma base $1, \theta, \theta^{2}, \ldots$, em que $r \theta=\theta \alpha(r)+\delta(r)$, para todo $r \in R$.

Definição 3.4.3. Seja $R[\theta ; \alpha, \delta]$ um anel de polinômios torcido. Qualquer elemento $p$ não nulo em $R[\theta ; \alpha, \delta]$ pode ser expressado unicamente na forma $p=r_{n} \theta^{n}+r_{n-1} \theta^{n-1}+\ldots+r_{1} \theta+r_{0}$ para algum inteiro não negativo $n$ e alguns elementos $r_{i} \in R$ com $r_{n} \neq 0$. O inteiro $n$ é chamado de grau de $p$, deg $(p)=n$, e o elemento $r_{n}$ é o coeficiente líder de $p$. O elemento zero de $R[\theta ; \alpha, \delta]$ por definição tem grau $-\infty$ e coeficiente líder 0 . 
No caso em que $R$ é domínio e $\alpha$ é injetivo, $\operatorname{deg}(p q)=\operatorname{deg}(p)+\operatorname{deg}(q)$, para todos $p, q \in$ $R[\theta ; \alpha, \delta]$. Em particular, $R[\theta ; \alpha, \delta]$ é um domínio neste caso.

Um dominio de ideais à direita principais é um domínio no qual todos os ideais à direita são principais. Dominio de ideais principais à esquerda são definidos analogamente.

Teorema 3.4.1. Sejam $R$ um anel de divisão, $\alpha$ um endomorfismo de $R$ e $\delta$ uma $\alpha$-derivação de $R$. Então o anel de polinômios torcido $S=R[\theta ; \alpha, \delta]$ é um dominio de ideias à esquerda principais. Se a é um automorfismo de $R$ então $S$ também é um domínio de ideais à direita principais.

Demonstração. [GJ89], Cap. 1, Teorema 1.11, pág. 12.

Existe um teorema análogo ao teorema da Base de Hilbert, para um anel de polinômios torcido. Notemos que $\alpha$ deve ser um automorfismo de $R$, por hipótese.

Teorema 3.4.2. Sejam $R$ um anel, $\alpha$ um automorfismo de $R$, e $\delta$ uma $\alpha$-derivação de $R$. Se $R$ é um anel Noetheriano à direita (à esquerda), então o anel de polinômios torcido

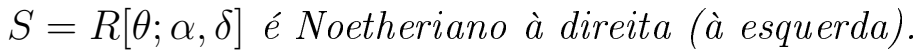

Demonstração. [GJ89], Cap. 1, Teorema 1.12, pág. 13.

Claramente, o Teorema 3.4.2 pode ser aplicado ao anel de polinômios torcido iterado, isto é, um anel da forma $R\left[\theta_{1} ; \alpha_{1}, \delta_{1}\right]\left[\theta_{2} ; \alpha_{2}, \delta_{2}\right] \cdots\left[\theta_{n} ; \alpha_{n}, \delta_{n}\right]$, em que $\alpha_{1}$ é um automorfismo de $R$ e $\delta_{1}$ é uma $\alpha_{1}$-derivação de $R$, enquanto $\alpha_{2}$ é um automorfismo de $R\left[\theta_{1} ; \alpha_{1}, \delta_{1}\right]$ e $\delta_{2}$ é uma $\alpha_{2}$-derivação de $R\left[\theta_{1} ; \alpha_{1}, \delta_{1}\right]$, etc.

Um caso particular interessante é um anel de operadores diferenciais iterado construído a partir de um anel $R$ e uma lista finita $\delta_{1}, \ldots, \delta_{n}$ de derivações comutantes em $R$, da seguinte maneira. Primeiramente construímos $R\left[\theta_{1} ; \delta_{1}\right]$. Então definimos a aplicação $\partial_{2}$ em $R\left[\theta_{1} ; \delta_{1}\right]$ de acordo com a regra $\partial_{2}\left(\sum r_{i} \theta_{1}^{i}\right)=\sum \partial_{2}\left(r_{i}\right) \theta_{1}^{i}$. Como $\delta_{2}$ comuta com $\delta_{1}$, cálculos rotineiros mostram que $\partial_{2}$ é uma derivação em $R\left[\theta_{1} ; \delta_{1}\right]$, e formamos o anel $R\left[\theta_{1} ; \delta_{1}\right]\left[\theta_{2} ; \partial_{2}\right]$. Similarmente $\delta_{3}$ se estende a uma derivação $\partial_{3}$ em $R\left[\theta_{1} ; \delta_{1}\right]\left[\theta_{2} ; \partial_{2}\right]$, e formamos o anel $R\left[\theta_{1} ; \delta_{1}\right]\left[\theta_{2} ; \partial_{2}\right]\left[\theta_{3} ; \partial_{3}\right]$. O anel de operadores diferenciais $R\left[\theta_{1} ; \delta_{1}\right]\left[\theta_{2} ; \partial_{2}\right] \cdots\left[\theta_{n} ; \partial_{n}\right]$, é denotado por $R\left[\theta_{1}, \ldots, \theta_{n} ; \delta_{1}, \ldots, \delta_{n}\right]$, e a menos de isomorfismo, ele independe da ordem na qual os $\delta_{i}$ 's são listados.

Definição 3.4.4. Seja $\delta$ uma derivação em um anel $R$. Um $\delta$-ideal de $R$ é qualquer ideal $I$ de $R$ tal que $\delta(I) \subseteq I$. O anel $R$ é chamado $\delta$-simples se os únicos $\delta$-ideais não nulos de $R$ são $\{0\}$ e $R$.

Vejamos agora uma construção direta, análoga a Proposição 3.4.1, em que dessa vez trabalharemos com endomorfismos aditivos do anel de polinômios de Laurent $R\left[x, x^{-1}\right]$, em que $\theta$ do anel de polinômios torcido, possui inverso.

Proposição 3.4.2. Sejam $R$ um anel e $\alpha$ um automorfismo de $R$. Então existe um anel $S$, contendo $R$ como subanel, com uma unidade $\theta \in S$ tal que $S$ é um $R$-módulo à esquerda livre com uma base da forma $1, \theta, \theta^{-1}, \theta^{2}, \theta^{-2}, \ldots$ e $\theta r=\alpha(r) \theta$, para todo $r \in R$.

Demonstração. Seja $E=\operatorname{End}_{\mathbb{Z}}\left(R\left[x, x^{-1}\right]\right)$, em que $x$ é uma indeterminada, e identifique $R$ em $E$ (como um subanel) via multiplicação à esquerda. Vamos estender $\alpha$ a um automorfismo de $R\left[x, x^{-1}\right]$ em que $\alpha\left(r x^{i}\right)=\alpha(r) x^{i}$, para todos $i \in \mathbb{Z}, r \in R$. Então definimos $\theta \in E$ de acordo com $\theta(f)=\alpha(f) x$, e observe que $\theta r=\alpha(r) \theta$, para todo $r \in R$. Além disso, $\theta$ é invertível em $E$, e $\theta^{-1}(f)=\alpha^{-1}(f) x^{-1}$, para todo $f \in R\left[x, x^{-1}\right]$.

Como na demonstração da Proposição 3.4.1, o conjunto $S=\sum_{i \in \mathbb{Z}} R \theta^{i}$ é um subanel de $E$, e as potências de $\theta$ são linearmente independentes sobre $R$. 
Definição 3.4.5. Sejam $R$ um anel e $\alpha$ um automorfismo de $R$. $O$ anel $S$ construído na Proposição 3.4.2 é denotado por $R\left[\theta, \theta^{-1} ; \alpha\right]$ e é chamado de anel de Laurent torcido. Note que se $\alpha$ é a identidade em $R$, então $R\left[\theta, \theta^{-1} ; \alpha\right]$ é o anel de polinômios de Laurent ordinário sobre $R$. Note também que o subanel de $R\left[\theta, \theta^{-1} ; \alpha\right]$ gerado por $R$ e $\theta$ pode ser identificado com o anel de polinômios torcido $R[\theta ; \alpha]$.

$A=A_{k, \mathrm{I}}$ é a álgebra de Weyl de posto infinito, cf. Capítulo 1, Seção 1.3. Vamos considerar que $k$ é um corpo que tem característica zero e é algebricamente fechado e I é um conjunto infinito enumerável em que $|\mathrm{I}|<|k|$. Seja $t_{i}=X_{i} Y_{i}=x_{i} \partial i$, para $i \in \mathrm{I}$.

Para $\mathrm{J} \subseteq \mathrm{I}$, seja $\theta_{\mathrm{J}}$ o automorfismo de $A$ dado por:

$$
\left\{\begin{array}{lllll}
\theta_{\mathrm{J}}\left(X_{j}\right)=Y_{j} & e & \theta_{\mathrm{J}}\left(Y_{j}\right)=-X_{j} & , \text { se } & j \in \mathrm{J} \\
\theta_{\mathrm{J}}\left(X_{i}\right)=X_{i} & e & \theta_{\mathrm{J}}\left(Y_{i}\right)=Y_{i} & \text {, se } & i \notin \mathrm{J}
\end{array}\right.
$$

Para $i \in \mathrm{I}$ temos:

$$
\theta_{\mathrm{J}}\left(t_{i}\right)=\left\{\begin{array}{lll}
-t_{i}-1 & , \text { se } & i \in \mathrm{J} \\
t_{i} & , \text { se } & i \notin \mathrm{J}
\end{array}\right.
$$

Para um $A$-módulo à esquerda $M$, o módulo torcido obtido da ação de $A$ em $M$ por $\theta_{\mathrm{J}}$ será denotado por $M^{\theta_{\mathrm{J}}}$. Então $M=M^{\theta_{\mathrm{J}}}$ como $k$-espaços vetoriais e a diferença entre os dois reside na ação de $A$ em $M^{\theta_{\mathrm{J}}}$.

Pelo descrito acima temos que $M^{\theta_{\mathrm{J}}}$ é um módulo de peso se, e somente se, $M$ é. Ou seja, $M=\bigoplus_{\mathbf{p} \in k^{\mathrm{I}}} M_{\mathbf{p}}$ se, e somente se, $M^{\theta_{\mathrm{J}}}=\bigoplus_{\theta_{\mathrm{J}}(\mathbf{p}) \in k^{\mathrm{I}}} M_{\theta_{\mathrm{J}}(\mathbf{p})}$. Além disso, $\mathbf{p} \in \operatorname{supp}(M)$ se, e

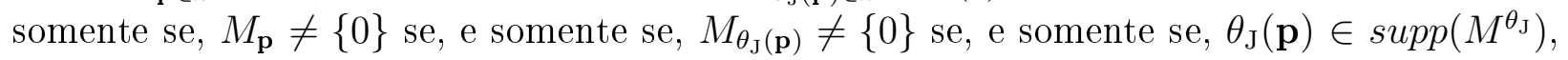
em que $\theta_{\mathrm{J}}(\mathbf{p})_{i}=p_{i}$ se $i \notin \mathrm{J}$ e $\theta_{\mathrm{J}}(\mathbf{p})_{i}=-p_{i}-1$ se $i \in \mathrm{J}$.

Denotemos por $k_{+}^{\mathrm{I}}$ o conjunto de todo $\mathbf{p} \in k^{\mathrm{I}}$ tal que se $p_{i} \in \mathbb{Z}$ implica que $p_{i} \in$ $\{0,1,2, \ldots\}$, para todo $i$. Para $\mathbf{p} \in k_{+}^{\mathrm{I}}$ denotemos por $\mathrm{J}_{\mathbf{p}}$ o conjunto de todo $i \in \mathrm{I}$ tal que $p_{i} \in \mathbb{Z}$.

Pelo Teorema 3.3.1 obtemos imediatamente o seguinte resultado:

Corolário 3.4.1. 1. Seja L um A-módulo de peso simples. Então existe um único $\mathbf{p} \in$ $k_{+}^{\mathrm{I}}$ e $\mathrm{J} \subseteq \mathrm{J}_{\mathbf{p}}$ tal que $L \cong L(\mathbf{p})^{\theta_{\mathrm{J}}}$. De fato, se $L=L(\mathbf{p})$, então $\mathrm{J}=\left\{i \in \mathrm{I}: q_{i} \in\right.$ $\{-1,-2, \ldots\}\}$ e $\mathbf{p}=\theta_{\mathrm{J}}(\mathbf{q})$.

2. Para $\mathbf{p}, \mathbf{q} \in k_{+}^{\mathrm{I}}, \mathrm{J} \subseteq \mathrm{J}_{\mathbf{p}}$ e $\mathrm{J}^{\prime} \subseteq \mathrm{J}_{\mathbf{q}}$ temos que $L(\mathbf{p})^{\theta_{\mathrm{J}}} \cong L(\mathbf{q})^{\theta_{\mathrm{J}^{\prime}}}$ se, e somente se, $\mathbf{p}=$ $\mathbf{q} e \mathrm{~J}=\mathrm{J}^{\prime}$.

Combinando o Corolário 3.4.1 com a Proposição 2.7.1 obtemos:

Corolário 3.4.2. Sejam $\mathbf{p} \in k_{+}^{\mathrm{I}} e \mathrm{~J} \subseteq \mathrm{J}_{\mathbf{p}}$. Entãa $B(\mathbf{p})^{\theta_{\mathrm{J}}}$ e $\left(B(\mathbf{p})^{\theta_{\mathrm{J}}}\right)^{\vee}$ são o envelope injetivo e a cobertura projetiva de $L(\mathbf{p})^{\theta_{\mathrm{J}}}$, respectivamente.

\subsection{Localização das realizações}

Basearemos esta seção em [FGM14][Seção 3.7]. Vamos continuar utilizando as notações da seção anterior.

Seja $\mathrm{J} \subseteq \mathrm{I}$. A ação adjunta de $X_{i}$ em $A$ é localmente nilpotente e portanto $X_{i}, i \in \mathrm{J}$, gera um subconjunto multiplicativo de Ore de $A$ dando origem à correspondente localização de Ore $\mathbf{D}_{\mathrm{J}} A$ de $A$ com respeito a esse subconjunto. Definimos o funtor $F_{\mathrm{J}}:=\operatorname{Res}_{A}^{\mathbf{D}_{\mathrm{J}} A} \circ \operatorname{Ind} d_{A}^{\mathbf{D}_{\mathrm{J}} A}$ na categoria $A$-mod. 
Além disso, similarmente à [Mat00][Lema 4.3], a álgebra $\mathbf{D}_{\mathrm{J}} A$ tem uma família de automorfismos $\varphi_{\mathbf{x}}, \mathbf{x} \in k^{\mathrm{J}}$, que são polinômios nas componentes de $\mathbf{x}$ e tais que para $\mathbf{x} \in \mathbb{Z}^{\mathrm{J}} \mathrm{e}$ para qualquer $a \in A$ temos que:

$$
\varphi_{\mathbf{x}}(a)=\prod_{i \in \mathrm{J}} X_{i}^{-x_{i}} a \prod_{i \in \mathrm{J}} X_{i}^{x_{i}}
$$

Observamos que se $a$ está fixo, então ele comuta com todos, mas uma quantidade finita dos $X_{i}$ 's, e então a expressão faz sentido por cancelar todos os outros termos.

Para um $\mathbf{D}_{\mathrm{J}} A$-módulo $M$, denotamos por $M^{\varphi_{\mathbf{x}}}$ o $\mathbf{D}_{\mathrm{J}} A$-módulo obtido de $M$ depois de torcido por $\varphi_{\mathbf{x}}$. Notemos que $\operatorname{supp}\left(M^{\varphi_{\mathbf{x}}}\right)=\operatorname{supp}(M)+\mathbf{x}$, em que $\mathbf{x}$ é considerado como um elemento de $k^{\mathrm{I}}$ definindo todas as componentes em $\mathrm{I} \backslash \mathrm{J}$ como zero.

Proposição 3.5.1. Seja $\mathbf{p} \in k_{+}^{\mathrm{I}}$.

1. $L(\mathbf{p}) \cong F_{\mathrm{I} \backslash \mathrm{J}_{\mathbf{p}}}^{\mathbf{p}^{\prime}} L(\mathbf{0})$, em que $\mathbf{p}^{\prime} \in k^{\mathrm{I} \backslash \mathrm{J}_{\mathbf{p}}}$ com $p_{i}^{\prime}=p_{i}$.

2. $B(\mathbf{p}) \cong F_{\mathrm{J}_{\mathbf{p}}} L(\mathbf{p})$.

Demonstração. Pelo Teorema 3.3.1, $L \mapsto \operatorname{supp}(L)$ define uma bijeção entre o conjunto dos $A$-módulos de peso simples e $k^{\mathrm{I}} / \sim$. Como todo módulo simples tem multiplicidades de peso no máximo 1 , para um $A$-módulo de peso $M$ temos que $M \cong L(\mathbf{p})$ se, e somente se, $\operatorname{supp}(M)=\operatorname{supp}(L(\mathbf{p}))$. Por outro lado, $\operatorname{supp}\left(F_{\mathrm{I} \backslash \mathrm{J}_{\mathbf{p}}}^{\mathbf{p}^{\prime}} L(\mathbf{0})\right)=\overline{\mathbf{p}}=\operatorname{supp}(L(\mathbf{p}))$, que implica 1 . Para provar 2. vamos usar a descrição polinomial de $B(\mathbf{p})$ da Subseção 2.7. Uma base vetorial de $F_{\mathrm{J}_{\mathbf{p}}} L(\mathbf{p})$ pode ser expressa unicamente na forma $\mathbf{x}^{\mathbf{m}} \mathbf{x}^{\mathbf{q}}$, em que $m_{i} \in\{0,-1,-2, \ldots\}$ se $i \in \mathrm{J}_{\mathbf{p}}, m_{j}=0$ se $j \notin \mathrm{J}_{\mathbf{p}}$, e $\mathbf{q} \in \overline{\mathbf{p}}$. Então $\mathbf{x}^{\mathbf{m}} \mathbf{x}^{\mathbf{q}} \mapsto \mathbf{x}^{\mathbf{m}+\mathbf{q}}$ define um isomorfismo $F_{\mathrm{J}_{\mathbf{p}}} L(\mathbf{p}) \cong B(\mathbf{p})$.

Notemos que devido ao Corolário 3.4.1, o envelope injetivo de todo módulo de peso simples $L$ é isomorfo à localização de $L$ com relação à um subconjunto multiplicativo de Ore de $A$ apropriado. Apresentar os injetivos indecomponíveis como localizações dos seus submódulos simples é um ideia explorada para categorias de módulos de peso de álgebras de Lie simpléticas em [GS06].

\subsection{Descrição explícita do quiver de $\mathfrak{W}$}

Basearemos esta seção em [FGM14][Seção 4.1].

Para um conjunto não vazio $E$ definimos o quiver $Q=Q_{E}$ como segue: os vértices de $Q$ são todos subconjuntos finitos de $E$. Para $U, W \in Q$ existe uma flecha de $U$ para $W$ se, e somente se, a diferença simétrica $U \triangle W$ é um conjunto unitário.

Lembremos que a diferença simétrica entre dois conjuntos $A$ e $B$, denotada por $A \triangle B$, é a união dos dois conjuntos menos a interseção entre eles, ou seja, $A \triangle B=(A \backslash B) \cup(B \backslash A)$.

Vamos impor em $Q$ as seguintes relações: se $U$ e $W$ são tais que existem flechas $\alpha: U \rightarrow$ $W$ e $\beta: W \rightarrow U$, então $\alpha \beta=\beta \alpha=0$; Se $U, W, U^{\prime}, W^{\prime}$ são conjuntos diferentes tais que existem flechas $\alpha: U \rightarrow W, \beta: W \rightarrow W^{\prime}, \alpha^{\prime}: U \rightarrow U^{\prime}$ e $\beta^{\prime}: U^{\prime} \rightarrow W^{\prime}$, então $\beta \alpha=\beta^{\prime} \alpha^{\prime}$.

Exemplo 3.6.1. Seja $E=\{1,2\}$. Os subconjuntos de $E$, que no caso serão os vértices de $Q_{E}$, são $\emptyset,\{1\},\{2\}$ e $\{1,2\}$. Temos que existe uma flecha entre $\emptyset$ e $\{1\}$, pois $\emptyset \triangle\{1\}=$ $\emptyset \cup\{1\} \backslash(\emptyset \cap\{1\})=\{1\} \backslash \emptyset=\{1\}$, porém não existe flecha entre $\{1\}$ e $\{2\}$, pois $\{1\} \triangle\{2\}=$ 
$\{1\} \cup\{2\} \backslash(\{1\} \cap\{2\})=\{1,2\} \backslash \emptyset=\{1,2\}$. Portanto o quiver $Q=Q_{E}$ correspondente é o seguinte:

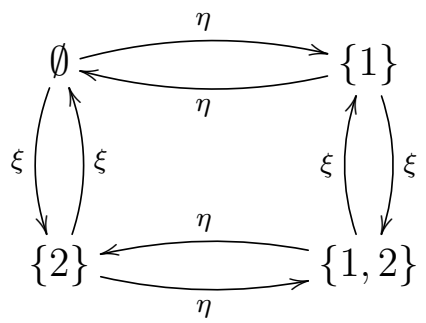

As relações de $Q_{E}$ s $\tilde{a} o: \eta^{2}=\xi^{2}=0$ e $\eta \xi=\xi \eta$.

Seja $Q=Q_{E}$ um quiver, em que $E$ é um conjunto não vazio. A categoria dos caminhos de $Q$ com as relações descritas acima será denotada por $C_{E}$. No caso a categoria de um quiver $Q$ tem como objetos os vértices e os morfismos entre dois objetos são os caminhos entre dois vértices dados.

A categoria $C_{E}$ é canonicamente isomorfa ao produto tensorial $\bigotimes_{i \in E} C_{i}$, tal que no caso de $E$ ser infinito o produto tensorial é entendido como o limite direto do sistema direcionado formado por todos os produtos tensoriais com respeito aos subconjuntos finitos de $E$ (veja [Bla77]). Notemos que a álgebra $C_{\{1\}}$ é a categoria de caminhos do seguinte quiver com relações:

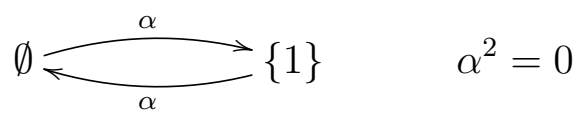

É fácil ver que no caso em que $E$ é finito todos os $C_{E}$-módulos projetivos são injetivos e temos o mesmo comprimento de Loewy.

\subsection{Koszulidade}

Basearemos esta seção em [FGM14][Seção 4.2].

Seguem algumas definições e comentários importantes. Para maiores detalhes indicamos [ASS06].

Definição 3.7.1 (Resolução projetiva). Uma resolução projetiva de um $R$-módulo $M$ é uma sequência exata $\ldots \longrightarrow P_{n} \stackrel{d_{n}}{\longrightarrow} P_{n-1} \longrightarrow \ldots \longrightarrow P_{1} \stackrel{d_{1}}{\longrightarrow} P_{0} \stackrel{d_{0}}{\longrightarrow} M \longrightarrow 0$ tal que cada $P_{i}$ é um $R$-módulo projetivo. Tal resolução é dita minimal se $d_{0}: P_{0} \rightarrow M$ e $d_{i}$ : $P_{i} \rightarrow \operatorname{ker}\left(d_{i-1}\right), \forall i \geq 1$, forem coberturas projetivas. Uma resolução projetiva é dita de comprimento $n$ se $P_{n} \neq\{0\}$ e $P_{i}=\{0\}, \forall i>n$. Neste caso, se $n$ for o menor inteiro com essa propriedade, dizemos que $M$ tem dimensão projetiva igual a $n$ e denotamos por $d p(M)$.

Sejam $Q$ um quiver finito, em que $Q_{0}=\{1, \ldots, n\}, k Q$ sua álgebra de caminhos e $e_{i} \mathrm{o}$ caminho trivial associado ao vértice $i \in Q_{0}$. Então $k Q$ é uma álgebra associativa e o conjunto $\left\{e_{i}\right\}_{i=1}^{n}$ é um sistema completo de idempotentes ortogonais primitivos de $k Q$. Em particular , $k Q$ tem identidade $1=e_{1}+\cdots+e_{n}$. Além disso, $k Q=e_{1}(k Q) \oplus \cdots e_{n}(k Q)$ é a decomposição de $k Q$ em módulos projetivos indecomponíveis, dois a dois não isomorfos; e $k Q$ tem dimensão finita se, e somente se, $Q$ não possui ciclos orientados.

Se $I$ é um ideal de $k Q$ gerado por combinações lineares de caminhos de comprimento dois, dizemos que $\bar{Q}=k Q / I$ é uma álgebra quadrática. Por outro lado, se cada gerador de $I$ for um caminho de $Q$, dizemos que $\bar{Q}$ é uma álgebra monomial. 
Seja $k Q$ a álgebra de caminhos de um quiver $Q$ sobre $k$. Para cada $n \geq 0$, denotamos por $(k Q)_{n}$ o $k$-espaço vetorial gerado pelos caminhos de comprimento $n$ em $Q$. Então:

$$
k Q=\bigoplus_{i \geq 0}(k Q)_{i}
$$

é uma $k$-álgebra graduada, em que $(k Q)_{n}(k Q)_{m} \subseteq(k Q)_{n+m}, \forall m, n \geq 0$.

Seja $R=\bigoplus_{i>0} R_{i}$ um anel graduado tal que $R_{0}$ é um anel semissimples, então dizemos que o ideal $\underline{r}=\bigoplus_{i \geq 1} R_{i}$ é o radical de Jacobson graduado de $R$. Em outras palavras, é a intersecção dos ideais maximais graduados de $R$.

Sejam $M, P R$-módulos graduados, $p: P \rightarrow M$ um epimorfismo de grau 0, ou seja, $p\left(P_{n}\right) \subseteq M_{n}$ para todo $n \geq 0$, e $P$ um $R$-módulo projetivo tal que $k e r(p) \subseteq \operatorname{rad}(P)=P \underline{r}$. Então o par $(P, p)$ é chamado de cobertura projetiva graduada de $M$.

Definição 3.7.2 (Resolução projetiva graduada). Seja $M$ um R-módulo graduado finitamente gerado. Por uma resolução projetiva graduada para $M$, entendemos uma sequência exata $\ldots \longrightarrow P_{n} \stackrel{d_{n}}{\longrightarrow} P_{n-1} \longrightarrow \ldots \longrightarrow P_{1} \stackrel{d_{1}}{\longrightarrow} P_{0} \stackrel{d_{0}}{\longrightarrow} M \longrightarrow 0$ em que, para cada $i \geq 0, P_{i}$ é um $R$-módulo projetivo graduado e cada aplicação $d_{i}$ é um $R$-homomorfismo de grau 0. Se além disso, $i m\left(d_{n}\right) \subseteq P_{n-1} \underline{r}$, para todo $n \geq 1$, então a resolução acima é dita minimal.

Definição 3.7.3 (Resolução linear). Seja $M$ um R-módulo graduado finitamente gerado. Suponha que $\ldots \longrightarrow P_{n} \stackrel{d_{n}}{\longrightarrow} P_{n-1} \longrightarrow \ldots \longrightarrow P_{1} \stackrel{d_{1}}{\longrightarrow} P_{0} \stackrel{d_{0}}{\longrightarrow} M \longrightarrow 0$ é uma resolução projetiva graduada de $M$. Dizemos que esta resolução é linear de comprimento $n$ se para todo $0 \leq i \leq n, P_{i}$ é gerado em grau $i$. Por outro lado, dizemos que $M$ tem resolução linear se existe uma resolução projetiva graduada

$$
\ldots \longrightarrow P_{n} \stackrel{d_{n}}{\longrightarrow} P_{n-1} \longrightarrow \ldots \longrightarrow P_{1} \stackrel{d_{1}}{\longrightarrow} P_{0} \stackrel{d_{0}}{\longrightarrow} M \longrightarrow 0
$$

que é linear para cada $n \geq 0$.

Toda resolução linear é minimal.

Recordemos que uma $k$-álgebra associativa $\mathbb{Z}$-graduada $C=\bigoplus_{i \in \mathbb{Z}} C_{i}$ é chamada $K o s z u l$ se $C_{0}$ é semissimples, $C_{i}=\{0\}$ se $i<0$, e a $i$-ésima componente da resolução projetiva graduada minimal de $C_{0}$ é gerada em grau $i$ (tal resolução é chamada linear). Similarmente definimos Koszulidade para categorias $k$-lineares.

Proposição 3.7.1. Seja E como na Seção 3.6. Então a categoria $C_{E}$ é Koszul.

Demonstração. Para $t \in E$ a álgebra $C_{\{t\}}$ é dada por 3.1 (Subseção 3.6). Ela é quadrática e monomial, então é Koszul (é muito simples escrever as resoluções projetivas lineares dos $C_{\{t\}^{-}}$ módulos simples). O resultado agora segue da observação que qualquer produto tensorial de álgebras Koszul é Koszul. Para ver este último fato, fixemos uma resolução linear para cada módulo simples sobre cada fator tensorial do produto. Tensoriando estas resoluções juntas (cada resolução por fator tensorial) e tomando o complexo total de maneira usual obtemos resoluções projetivas lineares para $C_{E^{-}}$módulos simples. O caso com uma quantidade finita de fatores pode ser encontrada em [BF85]. O caso infinito segue tomando o limite direto com respeito ao sistema direcionado dado pelos subconjuntos finitos de $E$. 


\subsection{Descrição dos blocos}

Finalmente, vamos mostrar que os blocos de $\mathfrak{W}$ são descritos por $C_{E}$ para $E$ apropriado. Recordemos que para $\mathbf{p} \in k_{+}^{\mathrm{I}}$ denotamos por $\mathrm{J}_{\mathbf{p}}$ o conjunto de todo $i \in \mathrm{I}$ tal que $p_{i} \in \mathbb{Z}$. Se $\mathrm{J}_{\mathbf{p}}=\emptyset$, o módulo projetivo $P(\mathbf{p})$ é simples, o que significa que $\mathfrak{W}_{\mathbf{p}}$ é semissimples e então é isomorfa à $k$-mod.

Teorema 3.8.1. Se $\mathrm{J}_{\mathbf{p}} \neq \emptyset$, então a categoria $\mathfrak{W}_{\mathbf{p}}$ é equivalente a categoria dos módulos sobre $C_{\mathrm{J}_{\mathrm{p}}}$.

Demonstração. Fixemos algum objeto representativo em cada classe de isomorfismos de projetivos indecomponíveis em $\mathfrak{W}_{\mathrm{p}}$ e seja $\mathcal{X}$ a subcategoria plena de $\mathfrak{W}_{\mathrm{p}}$ que é gerada por estes representantes fixos. Para provar nosso teorema aplicaremos a teoria clássica de Morita para anéis com unidades locais, veja [Abr83][Teorema 4.2]. A única coisa não trivial que temos que verificar é que $\mathcal{X}$ é isomorfa à $C_{\mathrm{J}_{\mathbf{p}}}$.

Para $\mathbf{m} \in \mathbf{p}+\mathbb{Z}_{f}^{\mathrm{I}}$, seja $U(\mathbf{m})$ o conjunto de todo $i \in \mathrm{J}_{\mathbf{p}}$ satisfazendo uma das duas condições: $p_{i} \in\{-1,-2, \ldots\}$ enquanto $m_{i} \in\{0,1,2, \ldots\}$ ou $p_{i} \in\{0,1,2, \ldots\}$ enquanto $m_{i}$ $\in\{-1,-2, \ldots\}$. Então a aplicação $P(\mathbf{m}) \mapsto U(\mathbf{m})$ induz uma bijeção de objetos de $\mathcal{X}$ em objetos de $C_{\mathrm{J}_{\mathbf{p}}}$. Encontramos a inversa desta aplicação da seguinte maneira: para um conjunto finito $U \subset \mathrm{J}_{\mathbf{p}}$ definimos $\mathbf{p}^{(U)}$ como segue:

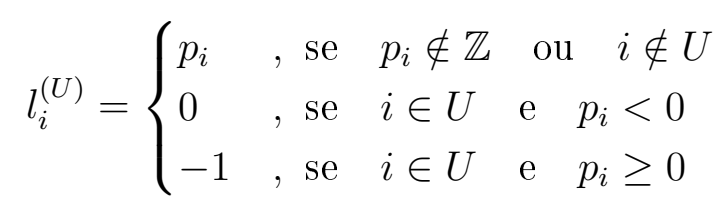

Para cada $U$ fixemos algum $v_{U} \in P\left(\mathbf{p}^{(U)}\right)_{\mathbf{p}^{(U)}}$ não nulo.

Tomemos agora algum conjunto finito $U \subset \mathrm{J}_{\mathbf{p}}$ e $i \in \mathrm{I}_{\mathbf{p}} \backslash U$.

Se $p_{i}<0$, então definimos o homomorfismo $\alpha_{U, i}: P\left(\mathbf{p}^{(U)}\right) \rightarrow P\left(\mathbf{p}^{(U \cup\{i\})}\right)$ enviando $v_{U}$ em $Y^{-p_{i}} v_{U \cup\{i\}}$, e definimos também o homomorfismo $\beta_{U, i}: P\left(\mathbf{p}^{(U \cup\{i\})}\right) \rightarrow P\left(\mathbf{p}^{(U)}\right)$ enviando $v_{U \cup\{i\}}$ em $X^{-p_{i}} v_{U}$.

Se $p_{i}>0$, então definimos o homomorfismo $\alpha_{U, i}: P\left(\mathbf{p}^{(U)}\right) \rightarrow P\left(\mathbf{p}^{(U \cup\{i\})}\right)$ enviando $v_{U}$ em $X^{p_{i}+1} v_{U \cup\{i\}}$, e definimos também o homomorfismo $\beta_{U, i}: P\left(\mathbf{p}^{(U \cup\{i\})}\right) \rightarrow P\left(\mathbf{p}^{(U)}\right)$ enviando $v_{U \cup\{i\}}$ em $Y^{p_{i}+1} v_{U}$.

É fácil verificar que esses homomorfismos satisfazem as relações definidas em $C_{\mathrm{J}_{\mathbf{p}}}$. Comparando os elementos dos módulos projetivos indecomponíveis em $\mathfrak{W}_{\mathbf{p}}$ e sobre $C_{\mathrm{J}_{\mathbf{p}}}$, concluímos que estas são todas as relações que os definem. O resultado segue.

\subsection{Referências}

Baseamos as Seções 3.1 e 3.2 em [BBF04][Seções 2, 3, 4 e 7], enquanto que as Seções 3.3, 3.4, 3.5, 3.6, 3.7 e 3.8, foram baseadas em [FGM14][Seções 3.5, 3.6, 3.7, 4.1, 4.2 e 4.3]. 
CLASSIFICAÇÃO 


\section{Apêndice A}

\section{Álgebras de Lie}

As álgebras de Lie surgiram com Sophus Lie (1842-1899) na década de 1870. Elas nasceram da tentativa de obter uma teoria para o estudo das equações diferenciais análogo à teoria de Galois para equações polinomiais. Utilizando as chamadas transformações de contato, Sophus Lie examinou um processo, criado por Jacobi, de obtenção de novas soluções de equações diferenciais a partir de uma dada solução. Isso levou Lie a tratar seus grupos de transformações (denominados atualmente grupos de Lie) através do que hoje chamamos "álgebra de Lie" (ou grupos infinitesimais). Uma das características da teoria de Lie é contrapor os conceitos complementares de grupos e álgebras de Lie.

Depois da introdução, a teoria das álgebras de Lie foi desenvolvida por Wilhelm Killing (1847-1923) e Élie Cartan (1869-1951).

\section{A.1 Conceitos Básicos}

Antes de falar sobre as álgebras de Lie vamos definir alguns conceitos e enunciar alguns resultados que foram úteis ao longo do presente trabalho. Para mais detalhes das estruturas algébricas elementares, homomorfismos e os teoremas relacionados recomendamos ao leitor [Rot03] e [Lam01].

Definição A.1.1. Um anel $R$ é um grupo abeliano aditivo munido com um produto $R \times$ $R \longrightarrow R$, denotado por $(a, b) \mapsto a b$, tal que para todos $a, b, c \in R$,

1. $a(b c)=(a b) c$;

2. $a(b+c)=a b+a c \quad e(b+c) a=b a+c a$;

3. Existe $1_{R} \in R$ tal que $1_{R} a=a=a 1_{R}$.

Se além disso, ab $=b a$, para todos $a, b \in R$, dizemos que o anel é comutativo.

Definição A.1.2. Um ideal à esquerda $I$ de um anel $R$ é um subconjunto não vazio de $R$ que é subgrupo aditivo e é tal que $R I \subseteq I$, ou seja, para todos $a \in R$ e b $\in I$ implica que ab $\in I$. Analogamente, define-se ideal à direita. Um ideal é bilateral se é um ideal à esquerda e à direita. Um ideal $I$ é dito próprio se $I \neq R$ ou equivalentemente se $1_{R} \notin I$.

Definição A.1.3. Sejam $R$ um anel e $I$ um ideal bilateral de $R$. O grupo abeliano aditivo $R / I$ é um anel com produto $(a+I)(b+I)=a b+I$, para todo $a, b \in R$. Chamamos esse anel de anel quociente. 
Definição A.1.4. Um anel não nulo $R$ é dito simples se $\{0\}$ e $R$ são os únicos ideais bilaterais em $R$. Os ideais $\{0\}$ e $R$ são chamados de ideais triviais.

Definição A.1.5. Um homomorfismo de anéis é uma aplicação $f$ de um anel $R$ em um anel $S$ tal que:

1. $f(a+b)=f(a)+f(b)$, para todos $a, b \in R$;

2. $f(a b)=f(a) f(b)$, para todos $a, b \in R$;

3. $f\left(1_{R}\right)=1_{S}$.

Se $f$ é um homomorfismo de $R$ em $R$, é chamado de endomorfismo de $R$.

Lembremos que um endomorfismo de um anel é um automorfismo se for bijetor (isto é, se for um isomorfismo).

Definição A.1.6. Seja $R$ um anel comutativo. Um ideal $\mathfrak{p}$ de $R$ é dito primo se $\mathfrak{p} \neq R$ e se $a b \in \mathfrak{p} \Rightarrow a \in \mathfrak{p}$ ou $b \in \mathfrak{p}$. Um ideal $\mathfrak{m}$ de $R$ é dito maximal em $R$ se $\mathfrak{m} \neq R$ e toda vez que exista um outro ideal $I$ tal que $\mathfrak{m} \subseteq I \subseteq R$ então ou $I=\mathfrak{m}$ ou $I=R$.

Definição A.1.7. Um anel não nulo $R$ é um anel de divisão se todo elemento não nulo de $R$ possui inverso multiplicativo, ou seja, é uma unidade. Um anel de divisão comutativo é um corpo.

Teorema A.1.1. Seja $k$ um anel comutativo. Então as seguintes condições são equivalentes:

1. k é um corpo.

2. $\{0\}$ é um ideal maximal em $k$.

3. $k$ é um anel simples.

Definição A.1.8. Seja $R$ um anel não nulo. Se existir um inteiro positivo $n$ tal que, para todo $a \in R, n a=a+\ldots+a=0$ ( $n$ vezes), então o menor desses inteiros positivos será chamado de característica do anel $R$. Diremos, nesse caso, que $R$ tem característica positiva. Se não existir nenhum inteiro positivo com a propriedade acima, diremos que $R$ tem característica zero. Diremos que um corpo $k$ tem característica 0 e denotamos por char $(k)=0$ (resp. $p, p$ é um primo) se, como anel, tiver característica 0 (resp. p, p é um primo).

Definição A.1.9. Um elemento em um anel $R$ é dito regular se não é um divisor de zero nem à direita, nem à esquerda de $R$.

Definição A.1.10. Um anel não nulo $R$ é dito ser um domínio se todos os seus elementos não nulos são regulares. Em outras palavras, se $a b=0$ implica $a=0$ ou $b=0$ em $R$, ou aind $a, a \neq 0, b \neq 0$ implica $a b \neq 0$. Se além disso, $R$ for um anel comutativo, então $R$ é chamado de domínio de integridade.

Proposição A.1.1. Seja $R$ um anel comutativo. pé um ideal primo de $R$ se, e somente se, $R / \mathfrak{p}$ é um domínio de integridade; $\mathfrak{m}$ é um ideal maximal em $R$ se, e somente se, $R / \mathfrak{m}$ é um corpo.

Demonstração. [Rot03], Capítulo 6, Proposição 6.4, pág. 321 e [Rot03], Capítulo 6, Proposição 6.7, pág. 322 . 
Definição A.1.11. Seja $R$ um anel. $U m$ R-módulo à esquerda é um grupo abeliano aditivo $M$ munido de uma aplicação $R \times M \rightarrow M$, que leva $(a, m) \mapsto$ am e que satisfaz:

1. $a(m+n)=a m+a n ;$

2. $(a+b) n=a n+b n$;

3. $(a b) m=a(b m)$;

4. $1_{R} m=m$.

para todos $a, b \in R$ e para todos $m, n \in M$. Define-se $R$-módulo à direita simetricamente. Se $R=k$ é um corpo, o k-módulo $M$ na verdade é um $k$-espaço vetorial.

Se $M$ é um $R$-módulo à esquerda (à direita) então um submódulo à esquerda (à direita) $N$ de $M$ é um subgrupo aditivo $N$ de $M$, fechado sob multiplicação por escalar: $r n \in N$, para todos $n \in N$ e $r \in R$. Se $N$ é um $R$-submódulo de $M$, então o módulo quociente é o grupo quociente $M / N$ ( $M$ é um grupo abeliano e $N$ é um subgrupo) equipado com a multiplicação por escalar $r(m+N)=r m+N$, para todos $r \in R, m \in M$.

Seja $M$ um $R$-módulo e $\left\{M_{i}: i \in I\right\}$ uma família de submódulos de $M$. O submódulo de $M$ gerado por $\bigcup_{i \in I} M_{i}$ é denotado por $\sum_{i \in I} M_{i}$ e, como é fácil ver, é formado por todos os elementos da forma $m_{1}+\ldots+m_{r}$, em que $r$ é um inteiro positivo e $m_{j} \in M_{i_{j}}$. Dizemos que $M$ é a soma direta de $\left\{M_{i}: i \in I\right\}$ se $M=\sum_{i \in I} M_{i}$ e para todos $j \in I$, temos que $M_{j} \cap\left(\sum_{i \neq j} M_{i}\right)=\{0\}$. Denotamos esse fato da seguinte maneira: $M=\bigoplus_{i \in I} M_{i}$. Se o conjunto $I$ for finito, digamos, $I=\{1, \ldots, r\}$, escrevemos $M=M_{1} \bigoplus \cdots \bigoplus M_{r}$ e nos referimos à essa igualdade como uma decomposição de $M$ em soma direta de submódulos. Nesse caso, cada elemento $m \in M$ se escreve de maneira única na forma $m=m_{1}+\cdots+m_{r}$, com $m_{j} \in M_{j}$, para todo $j=1, \ldots, r$.

Definição A.1.12. Sejam $M$ e $N$ R-módulos. Uma aplicação $f: M \rightarrow N$ é um homomorfismo de $R$-módulos (ou um $R$-homomorfismo) se:

1. $f\left(m_{1}+m_{2}\right)=f\left(m_{1}\right)+f\left(m_{2}\right)$;

2. $f\left(a m_{1}\right)=a f\left(m_{1}\right)$;

para todos $m_{1}, m_{2} \in M$ e para todo $a \in R$. Ou seja, $f$ é um homomorfismo de grupos abelianos aditivos que comuta com a ação de cada $a \in R$.

Seja $M$ um $R$-módulo e suponha que $M=M_{1} \oplus \cdots \bigoplus M_{r}$ seja uma decomposição de $M$ como soma direta de submódulos. Agora para cada $j$, considere as seguintes aplicações: $\pi_{j}: M \rightarrow M_{j}$, definida por $m \mapsto m_{j}$, e $\iota_{j}: M_{j} \rightarrow M$, definida por $m_{j} \mapsto m_{j}$. Então para cada $j, \pi_{j}$ é um homomorfismo sobrejetor, chamado projeção canônica sobre $M$ e $\iota_{j}$ é um homomorfismo injetor, chamado inclusão canônica de $M_{j}$.

Os homomorfismos canônicos definidos acima satisfazem as seguintes identidades:

$$
\sum_{j=1}^{r} \iota_{j} \pi_{j}=i d_{M} \quad \text { e } \quad \pi_{j} \iota_{k}=\delta_{j k} \cdot i d_{M_{k}}
$$

Agora vamos enunciar teoremas muito importantes da teoria dos módulos. Observamos que mutatis mutandis existem teoremas análogos para anéis. 
Teorema A.1.2 (Primeiro Teorema do Isomorfismo). Se $f: M \rightarrow N$ é um $R$-homomorfismo de módulos, então existe um R-isomorfismo

$$
\varphi: M / k e r(f) \rightarrow i m(f)
$$

Demonstração. [Rot03], Capítulo 7, Teorema 7.8, pág. 429.

Teorema A.1.3 (Segundo Teorema do Isomorfismo). Se $S$ e $T$ são submódulos de um $R$-módulo $M$, então existe um R-isomorfismo

$$
S /(S \cap T) \rightarrow(S+T) / T
$$

Demonstração. [Rot03], Capítulo 7, Teorema 7.9, pág. 429.

Teorema A.1.4 (Terceiro Teorema do Isomorfismo). Se $T \subseteq S \subseteq M$ é uma torre de $R$-submódulos, então existe um $R$-isomorfismo

$$
(M / T) /(S / T) \rightarrow M / S
$$

Demonstração. [Rot03], Capítulo 7, Teorema 7.10, pág. 430.

Teorema A.1.5 (Teorema da Correspôndencia). Se $T$ é um submódulo de um R-módulo $M$, então existe uma bijeção

$\varphi:\{$ submódulos intermediários $: T \subseteq S \subseteq M\} \rightarrow\{$ submódulos de $M / T\}$

dado por $S \mapsto S / T$. Além disso, $S \subseteq S^{\prime}$ em $M$ se, e somente se, $S / T \subseteq S^{\prime} / T$ em $M / T$.

Demonstração. [Rot03], Capítulo 7, Teorema 7.11, pág. 430.

Definição A.1.13. Seja $f: R \rightarrow S$ um homomorfismo de anéis. Se $a \in R$ e $b \in S$ defina $a b=f(a) b$. Logo $S$ tem estrutura de $R$-módulo e estrutura de anel. Chamamos ao anel $S$ equipado com sua estrutura de $R$-módulo de $R$-álgebra. Em outras palavras uma $R$-álgebra é um anel $S$ junto com um homomorfismo de anéis $f: R \rightarrow S$. Sendo assim uma álgebra sobre um corpo $k$ é um k-espaço vetorial (k-módulo) equipado com um produto bilinear, ou seja, é um conjunto munido com um produto, uma soma e uma multiplicação por escalares do corpo $k$.

Definição A.1.14. Se $f: R \rightarrow S$ e $g: R \rightarrow T$ são dois homomorfismos de anéis, um homomorfismo de $R$-álgebras $h: S \rightarrow T$ é um homomorfismo de anéis que também é um homomorfismo de R-módulos.

Definição A.1.15. Seja $k$ um corpo. Uma álgebra associativa sobre $k$ ou k-álgebra associativa é um espaço vetorial $\mathfrak{A}$ sobre $k$ munido de um produto $\mathfrak{A} \times \mathfrak{A} \rightarrow \mathfrak{A},(a, b) \mapsto a b$, tal que $a(b c)=(a b) c$, para todos $a, b, c \in \mathfrak{A}$.

Observação A.1.1. Uma álgebra é unital se possui elemento identidade com respeito ao produto. 


\section{A.2 Álgebras de Lie}

Agora vamos apresentar uma introdução à teoria das álgebras de Lie. Para mais detalhes recomendamos [Hum13], [Mar10], [Eti11] e [Sam90].

\section{A.2.1 Generalidades}

Definição A.2.1. Uma álgebra de Lie consiste de um espaço vetorial $\mathfrak{g}$ sobre um corpo $k$, munido de um produto (colchete ou comutador)

$$
[\cdot, \cdot]: \mathfrak{g} \times \mathfrak{g} \rightarrow \mathfrak{g}
$$

com as seguintes propriedades:

1. É bilinear;

2. É anti-simétrico, isto é, $[x, x]=0$ para todo $x \in \mathfrak{g}$ (o que implica $[x, y]=-[y, x]$ para todo $x, y \in \mathfrak{g}$ e é equivalente se o corpo dos escalares $k$ não tem característica dois);

3. Satisfaz a identidade de Jacobi, isto é, para todo $x, y, z \in \mathfrak{g}$,

$$
[x,[y, z]]+[z,[x, y]]+[y,[z, x]]=0 .
$$

Recordemos que $A_{n}(k)$, a $n$-ésima álgebra de Weyl sobre $k$, com geradores $x_{1}, \ldots, x_{n}$ e $\partial_{1}, \ldots, \partial_{n}$, satisfaz as seguintes relações: $\left[\partial_{i}, x_{j}\right]=\delta_{i j} \cdot 1,\left[\partial_{i}, \partial_{j}\right]=\left[x_{i}, x_{j}\right]=0$, para $1 \leq i, j \leq n$. Segue que podemos considerar $A_{n}(k)$ como uma álgebra de Lie sobre $k$.

Em geral, uma álgebra é um espaço vetorial $A$ sobre um corpo $k$ munido de um produto, isto é, uma aplicação de $A \times A$ para $A$. Qualquer aplicação deste tipo que mereça o nome de produto deve ser bilinear e usualmente é denotado por justaposição. A anti-simetria e a identidade de Jacobi são características das álgebras de Lie. Outros tipos de álgebras têm outros tipos de propriedades que a definem. Por exemplo, as álgebras associativas, satisfazem a seguinte propriedade adicional: $a(b c)=(a b) c$, como visto anteriormente.

Observação A.2.1. O colchete de Lie não é em geral associativo, pois em qualquer circunstância $[[x, x], y]=0$, e no entanto $[x,[x, y]]$ nem sempre se anula.

Definição A.2.2. A dimensão de uma álgebra de Lie $\mathfrak{g}$ é sua dimensão como espaço vetorial sobre um corpo $k$.

Definição A.2.3. Seja $\mathfrak{g}$ uma álgebra de Lie. Uma subálgebra de $\mathfrak{g}$ é um subespaço vetorial $\mathfrak{h}$ de $\mathfrak{g}$ que é fechado pelo colchete, isto é, $[\mathfrak{h}, \mathfrak{h}] \subset \mathfrak{h}$, ou ainda, $[x, y] \in \mathfrak{h}$ se $x, y \in \mathfrak{h}$.

Observação A.2.2. Qualquer elemento não nulo pertencente à $\mathfrak{g}$ define uma subálgebra unidimensional (no caso, o subespaço vetorial gerado por esse elemento) com o mesmo produto de $\mathfrak{g}$.

Dada uma álgebra associativa $\mathfrak{A}$, definimos $\mathfrak{A}^{(-)}$como a álgebra de Lie com o colchete definido por $[a, b]=a b-b a$, para todo $a, b \in \mathfrak{A}$.

Seja $V$ um espaço vetorial sobre um corpo $k$ de dimensão $n$. O conjunto de todas as transformações lineares de $V$ em $V$ é uma álgebra associativa, $E n d_{k}(V)$, com as operações de soma (definida ponto a ponto) e composição. Como $k$-espaço vetorial, $\operatorname{End}_{k}(V)$ tem dimensão $n^{2}$. Podemos identificar esse espaço vetorial com o espaço das matrizes $n \times n$ 
com coeficientes em $k$. Então $\operatorname{End}_{k}(V)^{(-)}$é uma álgebra de Lie, com o colchete definido da seguinte maneira: $[x, y]=x y-y x$, para todo $x, y \in \operatorname{End}_{k}(V)$. Denotamos essa álgebra de Lie por $\mathfrak{g l}(V)$. Ela é chamada de álgebra de Lie linear geral, pois é intimamente ligada ao grupo linear geral $G L(V)$, que consiste de todos os endomorfismos de $V$ que são inversíveis. No caso do espaço das matrizes, o colchete é definido da mesma forma, tais que $x, y$ são matrizes. Nesse último caso denotamos a álgebra de Lie por $\mathfrak{g l}(n, k)$ ou simplesmente $\mathfrak{g l}(n)$, quando o corpo não for relevante. A tábua de multiplicação para $\mathfrak{g l}(n, k)$ relativa à base canônica consistindo de matrizes $e_{i j}$ (com 1 na posição $(i, j)$ e 0 nas outras), em que $e_{i j} e_{k l}=\delta_{j k} e_{i l}$, é: $\left[e_{i j}, e_{k l}\right]=\delta_{j k} e_{i l}-\delta_{l i} e_{k j}$.

Qualquer subálgebra da álgebra de Lie $\mathfrak{g l}(V)$ é chamada de álgebra de Lie linear. Elas se dividem em quatro famílias $A_{l}, B_{l}, C_{l}$ e $D_{l}(l \geq 1)$ que são chamadas de álgebras de Lie clássicas, porque elas correspondem à certos grupos de Lie lineares clássicos. Para $B_{l}$ e $D_{l}$, seja $\operatorname{char}(k) \neq 2$.

1. $A_{l}$ : Seja $\operatorname{dim}(V)=l+1$. Denotemos por $\mathfrak{s l}(V)$, ou $\mathfrak{s l}(l+1, k)$, o conjunto dos endomorfismos de $V$ com traço zero. Recordemos que o traço de uma matriz $t r$ é a soma das entradas da sua diagonal principal. O traço independe da escolha da base de $V$, e por isso faz sentido para um endomorfismo de $V$. Como $\operatorname{tr}(x y)=\operatorname{tr}(y x)$ e $\operatorname{tr}(x+y)=\operatorname{tr}(x)+\operatorname{tr}(y)$, segue que $\mathfrak{s l}(V)$ é uma subálgebra de $\mathfrak{g l}(V)$, chamada de álgebra linear especial, por causa da sua conexão com o grupo linear especial $S L(V)$ dos endomorfismos com det igual à 1. Então:

$$
\mathfrak{s l}(l+1, k)=\{x \in \mathfrak{g l}(l+1, k) \mid \operatorname{tr}(x)=0\}
$$

Temos que $\operatorname{dim}(\mathfrak{s l}(l+1, k))=(l+1)^{2}-1$.

2. $C_{l}$ : Seja $\operatorname{dim}(V)=2 l$, com base $\left\{v_{1}, \ldots, v_{2 l}\right\}$. Definimos uma forma bilinear $f$ anti simétrica não degenerada de $V$ com matriz $s=\left(\begin{array}{cc}0 & I_{l} \\ -I_{l} & 0\end{array}\right)$. A dimensão par é uma condição necessária para a existência de uma forma bilinear não degenerada satisfazendo $f(v, w)=-f(w, v)$. Denotemos por $\mathfrak{s p}(V)$, ou $\mathfrak{s p}(2 l, k)$, a álgebra simplética, cuja definição consiste de todos os endomorfismos $x$ de $V$ que satisfazem $f(x(v), w)=$ $-f(v, x(w))$. Então:

$$
\mathfrak{s p}(2 l, k)=\left\{x \in \mathfrak{g l}(2 l, k) \mid s x+x^{t} s=0\right\}
$$

em que $x^{t}$ é a transposta de $x$. Temos que $\operatorname{dim}(\mathfrak{s p}(2 l, k))=2 l^{2}+l$.

3. $B_{l}$ : Seja $\operatorname{dim}(V)=2 l+1$ ímpar, e tome $f$, uma forma bilinear simétrica não degenerada, em $V$ cuja matriz é $s=\left(\begin{array}{ccc}1 & 0 & 0 \\ 0 & 0 & I_{l} \\ 0 & I_{l} & 0\end{array}\right)$. A álgebra ortogonal $\mathfrak{o}(V)$, ou $\mathfrak{o}(2 l+1, k)$, consiste de todos os endomorfismos $x$ de $V$ satisfazendo $f(x(v), w)=-f(v, x(w))$.

4. $D_{l(l \geq 2)}$ : Aqui obtemos outra álgebra ortogonal. A construção é idêntica à construção em $B_{l}$, exceto que a $\operatorname{dim}(V)=2 l$ é par e $s$ tem forma $s=\left(\begin{array}{cc}0 & I_{l} \\ I_{l} & 0\end{array}\right)$.

Exemplo A.2.1. Vejamos outras subálgebras de $\mathfrak{g l}(n, k)$ : seja $\mathfrak{t}(n, k)$ o conjunto das matrizes triangulares superiores $\left(a_{i j}\right), a_{i j}=0$ se $i>j$; seja $\mathfrak{n}(n, k)$, as matrizes estritamente triangulares superiores $\left(a_{i j}=0\right.$, se $\left.i \geq j\right)$; finalmente, seja $\mathfrak{d}(n, k)$ o conjunto das matrizes diagonais. Temos que $\mathfrak{t}(n, k), \mathfrak{n}(n, k)$ e $\mathfrak{d}(n, k)$ são subálgebras de $\mathfrak{g l}(n, k)$. Além disso, 
$\mathfrak{t}(n, k)=\mathfrak{d}(n, k)+\mathfrak{n}(n, k)$ (soma direta de espaços vetoriais), com $[\mathfrak{d}(n, k), \mathfrak{n}(n, k)]=\mathfrak{n}(n, k)$, $\log o[\mathfrak{t}(n, k), \mathfrak{t}(n, k)]=\mathfrak{n}(n, k)$.

\section{A.2.2 Derivações de álgebras de Lie}

Algumas transformações lineares de álgebras de Lie surgem naturalmente como derivações de álgebras. Lembremos que uma derivação de uma $k$-álgebra associativa $\mathfrak{A}$ é uma aplicação linear $\delta: \mathfrak{A} \rightarrow \mathfrak{A}$ satisfazendo a regra $\delta(a b)=a \delta(b)+\delta(a) b$. A coleção $\operatorname{Der}_{k}(\mathfrak{A})$ de todas as derivações de $\mathfrak{A}$ é um subespaço vetorial de $\operatorname{End}_{k}(\mathfrak{A})$. O comutador $\left[\delta, \delta^{\prime}\right]$ de duas derivações é uma derivação (embora o produto ordinário de duas derivações, no caso a composição, não seja necessariamente uma derivação). Então $\operatorname{Der}_{k}(\mathfrak{A})$ é uma subálgebra de $\mathfrak{g l}(\mathfrak{A})$.

Como uma álgebra de Lie $\mathfrak{g}$ é uma $k$-álgebra, faz sentido definirmos $\operatorname{Der}_{k}(\mathfrak{A})$. Certas derivações surgem naturalmente como segue. Se $x \in \mathfrak{g}, y \mapsto[x, y]$ é um endomorfismo de $\mathfrak{g}$, que denotamos por $a d(x)$. De fato, $a d(x) \in \operatorname{Der}_{k}(\mathfrak{A})$, porque podemos escrever a identidade de Jacobi na forma: $[x,[y, z]]=[[x, y], z]+[y,[x, z]]$, ou seja, $\operatorname{ad}(x)([y, z])=$ $[a d(x)(y), z]+[y, a d(x)(z)]$. Derivações dessa forma são chamadas internas, e todas as outras externas. É possível que $a d(x)=0$, enquanto $x \neq 0$; isto ocorre em alguma álgebra de Lie unidimensional, por exemplo. A aplicação $\left.\mathfrak{g} \rightarrow \operatorname{Der}_{k}(\mathfrak{A})\right)$, que envia $x$ em $\operatorname{ad}(x)$ é chamada de representação adjunta de $\mathfrak{g}$.

Algumas vezes $x$ é simultaneamente um elemento de $\mathfrak{g}$ e de uma subálgebra $\mathfrak{h}$ de $\mathfrak{g}$. Para evitar ambiguidade, a notação $a d_{\mathfrak{g}}(x)$ ou $a d_{\mathfrak{h}}(x)$ serão usadas para indicar que $x$ está agindo em $\mathfrak{g}$ ( respectivamente, $\mathfrak{h})$. Por exemplo, se $x$ é uma matriz diagonal, então $a d_{\mathfrak{d}(n, k)}(x)=0$, enquanto que $a d_{\mathfrak{g l}(n, k)}(x)$, não é necessariamente zero.

Vimos alguns exemplos naturais de álgebras de Lie lineares. Temos que, toda álgebra de Lie de dimensão finita é isomorfa à alguma álgebra de Lie linear.

Teorema A.2.1 (Teorema de Ado). Toda álgebra de Lie de dimensão finita $\mathfrak{g}$, de característica zero, tem uma representação de dimensão finita fiel.

Demonstração. [Jac79], Capítulo VI, pág. 202.

Algumas vezes é desejável, entretanto, contemplar álgebras de Lie abstratamente. Por exemplo, se g é um espaço vetorial arbitrário sobre $k$ de dimensão finita, podemos considerar $\mathfrak{g}$ como uma álgebra de Lie, em que $[x, y]=0$, para todos $x, y \in \mathfrak{g}$. Tal álgebra, com uma multiplicação de Lie trivial, é chamada de abeliana, porque no caso linear $[x, y]=0$ significa que $x$ e $y$ comutam.

\section{A.2.3 Ideais e homomorfismos}

Definição A.2.4. Um subespaço vetorial $\mathfrak{h}$ de uma álgebra de Lie $\mathfrak{g}$ é um ideal se para todo $y \in \mathfrak{h}, x \in \mathfrak{g}$, temos que $[x, y] \in \mathfrak{h}$.

Como $[x, y]=-[y, x]$, a condição poderia ser escrita $[y, x] \in \mathfrak{h}$.

É claro que todo ideal é subálgebra. Nem toda subálgebra, no entanto, é ideal. Vejamos:

Exemplo A.2.2. Consideremos o subespaço de $\mathfrak{s l}(2, \mathbb{R})$ gerado por $\left(\begin{array}{cc}1 & 0 \\ 0 & -1\end{array}\right)$. Esse subespaço é uma subálgebra por ser unidimensional. Não é, porém, um ideal pois

$$
\left[\left(\begin{array}{cc}
1 & 0 \\
0 & -1
\end{array}\right),\left(\begin{array}{ll}
0 & 1 \\
0 & 0
\end{array}\right)\right]=\left(\begin{array}{ll}
0 & 2 \\
0 & 0
\end{array}\right)
$$


Obviamente $\{0\}$ (o subespaço consistindo somente do vetor nulo) e $\mathfrak{g}$ são ideais de $\mathfrak{g}$. Um exemplo menos trivial é o centro de $\mathfrak{g}, Z(\mathfrak{g})=\{z \in \mathfrak{g} \mid[x, z]=0, \forall x \in \mathfrak{g}\}$. Notemos que $\mathfrak{g}$ é abeliana se, e somente se, $Z(\mathfrak{g})=\mathfrak{g}$. Outro importante exemplo é a álgebra derivada de $\mathfrak{g}$, denotada por $[\mathfrak{g}, \mathfrak{g}]$, que é análoga ao subgrupo comutador de um grupo. Ela consiste de todas as combinações lineares de comutadores $[x, y]$, e claramente é um ideal.

Evidentemente $\mathfrak{g}$ é abeliana se, e somente se, $[\mathfrak{g}, \mathfrak{g}]=\{0\}$. No outro extremo, um estudo da tábua de multiplicação para $\mathfrak{g}=\mathfrak{s l}(n, k)(n \neq 2$, se $\operatorname{char}(k)=2)$ mostra que $\mathfrak{g}=[\mathfrak{g}, \mathfrak{g}]$ neste caso, e similarmente para outras álgebras de Lie lineares clássicas (ver [Hum13], Capítulo $I$, exercício 1.9).

Se $\mathfrak{h}_{1}$ e $\mathfrak{h}_{2}$ são ideais de $\mathfrak{g}$ então

$$
\mathfrak{h}_{1}+\mathfrak{h}_{2}=\left\{x+y \mid x \in \mathfrak{h}_{1}, y \in \mathfrak{h}_{2}\right\}
$$

é também um ideal. Similarmente,

$$
\left[\mathfrak{h}_{1}, \mathfrak{h}_{2}\right]=\left\{\sum_{i, j} a_{i j}\left[x_{i}, y_{j}\right] \mid x_{i} \in \mathfrak{h}_{1}, y_{j} \in \mathfrak{h}_{2}, a_{i j} \in k\right\}
$$

é um ideal de $\mathfrak{g}$. A álgebra derivada $[\mathfrak{g}, \mathfrak{g}]$ é um caso especial desta construção.

É natural analisar a estrutura de uma álgebra de Lie olhando seus ideais. Se $\mathfrak{g}$ não tem ideais exceto ela mesma e $\{0\}$, e se além disso $[\mathfrak{g}, \mathfrak{g}] \neq\{0\}$, dizemos que $\mathfrak{g}$ é simples. A condição $[\mathfrak{g}, \mathfrak{g}] \neq\{0\}$ (isto é, $\mathfrak{g}$ não abeliana) é imposta com intuito de evitar dar destaque indevido à uma álgebra unidimensional. Claramente, $\mathfrak{g}$ simples implica que $Z(\mathfrak{g})=\{0\}$ e $[\mathfrak{g}, \mathfrak{g}]=\mathfrak{g}$.

Exemplo A.2.3. Seja $\mathfrak{g}=\mathfrak{s l}(2, k), \operatorname{char}(k) \neq 2$. Tomemos como base canônica para $\mathfrak{g}$ as três matrizes:

$$
x=\left(\begin{array}{ll}
0 & 1 \\
0 & 0
\end{array}\right) \quad, \quad y=\left(\begin{array}{ll}
0 & 0 \\
1 & 0
\end{array}\right) \quad \text { e } \quad h=\left(\begin{array}{cc}
1 & 0 \\
0 & -1
\end{array}\right)
$$

A tábua de multiplicação é completamente determinada pelas equações:

$$
[x, y]=h \quad, \quad[h, x]=2 x \quad \text { e } \quad[h, y]=-2 y
$$

Notemos que $x, y, h$ são autovetores para ad( $h)$, correspondendo aos autovalores $2,-2,0$, respectivamente. Como char $(k) \neq 2$, estes autovalores são distintos. Se $\mathfrak{g}^{\prime} \neq\{0\}$ é um ideal de $\mathfrak{g}$, seja $a x+b y+$ ch um elemento não nulo arbitrário de $\mathfrak{g}^{\prime}$. Aplicando ad $(x)$ duas vezes, temos $-2 b x \in \mathfrak{g}^{\prime}$, e aplicando ad(y) duas vezes, temos $-2 a y \in \mathfrak{g}^{\prime}$. Então, se a ou b é nã nulo, $\mathfrak{g}^{\prime}$ contém y ou $x(\operatorname{char}(k) \neq 2)$, e então por conta das equações acima, segue que $\mathfrak{g}^{\prime}=\mathfrak{g}$. Por outro lado, se $a=b=0$, então $0 \neq c h \in \mathfrak{g}^{\prime}$, e novamente por conta das equações, segue que $\mathfrak{g}^{\prime}=\mathfrak{g}$. Concluímos que $\mathfrak{g}=\mathfrak{s l}(2, k)$ é simples.

No caso que g não é simples (e não é unidimensional) é possível "extrair" um ideal próprio não nulo h e assim obter um álgebra de Lie de dimensão menor. A construção de uma álgebra quociente $\mathfrak{g} / \mathfrak{h}$ (h é um ideal de $\mathfrak{g}$ ) é formalmente a mesma construção de um anel quociente: como espaço vetorial $\mathfrak{g} / \mathfrak{h}$ é simplesmente o espaço quociente e sua multiplicação de Lie está bem definida por $[x+\mathfrak{h}, y+\mathfrak{h}]=[x, y]+\mathfrak{h}$, ou seja, $[\bar{x}, \bar{y}]=\overline{[x, y]}$, para todo $x, y \in \mathfrak{g}$.

Agora vamos mencionar uma dupla de noções relacionadas analogamente com o que ocorre na teoria dos grupos. O normalizador de uma subálgebra $\mathfrak{h}$ de uma álgebra de Lie $\mathfrak{g}$ é definido por $N_{\mathfrak{g}}(\mathfrak{h})=\{x \in \mathfrak{g} \mid[x, \mathfrak{h}] \subset \mathfrak{h}\}$. Pela identidade de Jacobi, $N_{\mathfrak{g}}(\mathfrak{h})$ é uma subálgebra de $\mathfrak{g}$ e é descrita como a maior subálgebra de $\mathfrak{g}$ que contém $\mathfrak{h}$ como ideal (inicialmente $\mathfrak{h}$ é só uma subálgebra). Se $\mathfrak{h}=N_{\mathfrak{g}}(\mathfrak{h})$, dizemos que $\mathfrak{h}$ é auto-normalizada. O centralizador de um 
subconjunto $\mathrm{X} \subseteq \mathfrak{g}$ é $C_{\mathfrak{g}}(X)=\{x \in \mathfrak{g} \mid[x, X]=0\}$. Novamente pela identidade de Jacobi, $C_{\mathfrak{g}}(X)$ é uma subálgebra de $\mathfrak{g}$. Por exemplo, $C_{\mathfrak{g}}(\mathfrak{g})=Z(\mathfrak{g})$.

Definição A.2.5. Uma transformação linear $\psi: \mathfrak{g} \rightarrow \mathfrak{h}$ (com $\mathfrak{g}$ e h álgebras de Lie sobre $k$ ) é $u m$ homomorfismo de álgebras de Lie se preserva o colchete, isto é, $\psi([x, y])=[\psi(x), \psi(y)]$, para todo $x, y \in \mathfrak{g}$.

$\psi$ é um monomorfismo se $\operatorname{ker}(\psi)=\{0\}$, é um epimorfismo se $i m(\psi)=\mathfrak{h}$ e um isomorfismo se é epimorfismo e monomorfismo.

Seja $V$ um espaço vetorial sobre um corpo $k$ e $\mathfrak{g l}(V)$ a álgebra de Lie dos endomorfismos de $V$. Seja $\mathfrak{g}$ uma álgebra de Lie (sobre o mesmo corpo de escalares de $V$ ). Uma representação de $\mathfrak{g}$ em $V$ é um homomorfismo $\rho: \mathfrak{g} \rightarrow \mathfrak{g l}(V)$.

Na terminologia usual, $V$ é o espaço da representação, enquanto que sua dimensão é a dimensão da representação. Uma representação $\rho$ é dita fiel se $k e r(\rho)=0$.

A noção de representar vem da ideia de descrever (representar) as álgebras de Lie como álgebras de transformações lineares. No caso das representações fiéis, $\mathfrak{g} \cong i m(\rho)$ e, portanto, a álgebra $\mathfrak{g}$ pode ser vista como uma subálgebra de $\mathfrak{g l}(V)$ (ou de $\mathfrak{g l}(n)$, se a dimensão é finita). Essa ideia de considerar álgebras de Lie como subálgebras de $\mathfrak{g l}(V)$ é realizada, pelo menos ao nível teórico, para as álgebras de Lie de dimensão finita, por conta do Teorema de Ado A.2.1.

Um exemplo importante de representação é a representação adjunta ad $: \mathfrak{g} \rightarrow \mathfrak{g l}(\mathfrak{g})$, definida anteriormente, que envia $x$ em $a d(x)$, tal que $a d(x)(y)=[x, y]$. Claramente ad é uma transformação linear. Além disso, preserva o colchete, ou seja, $[\operatorname{ad}(x), \operatorname{ad}(y)](z)=$ $a d([x, y])(z)$. Agora vamos analisar quem é o $\operatorname{ker}(a d)$. Ele consiste de todos $x \in \mathfrak{g}$ para os quais $\operatorname{ad}(x)=0$, isto é, para os quais $[x, y]=0$ (para todo $y \in \mathfrak{g})$. Então o $\operatorname{ker}(a d)=Z(\mathfrak{g})$. Isto tem uma interessante consequência: se $\mathfrak{g}$ é simples, temos que $Z(\mathfrak{g})=\{0\}$, então ad: $\mathfrak{g} \rightarrow \mathfrak{g l}(\mathfrak{g})$ é um monomorfismo. Isto significa que qualquer álgebra de Lie simples é isomorfa à uma álgebra de Lie linear.

Um automorfismo de $\mathfrak{g}$ é um isomorfismo de $\mathfrak{g}$ em $\mathfrak{g}$. Aut $(\mathfrak{g})$ denota o grupo de todos eles. Importantes exemplos ocorrem quando $\mathfrak{h}$ é uma álgebra de Lie linear $\subseteq \mathfrak{g l}(V)$. Se $g \in$ $G L(V)$ é algum endomorfismo inversível de $V$, e se além disso $g \mathfrak{h} g^{-1}=\mathfrak{h}$, então é imediato que a aplicação $x \mapsto g x g^{-1}$ é um automorfismo de $\mathfrak{h}$. Por exemplo, se $\mathfrak{h}=\mathfrak{g l}(V)$ ou até mesmo $\mathfrak{s l}(V)$, a condição $g \mathfrak{h} g^{-1}=\mathfrak{h}$ é automática, então obtemos desta maneira uma grande coleção de automorfismos.

\section{A.2.4 Álgebras de Lie Solúveis e Nilpotentes}

É natural estudar uma álgebra de Lie $\mathfrak{g}$ via seus ideais. Vamos explorar a formação das álgebras derivadas.

Primeiro, definimos uma sequência de ideais de $\mathfrak{g}$ (a série derivada) por:

$$
\mathfrak{g}^{(0)}=\mathfrak{g} \quad, \quad \mathfrak{g}^{(1)}=[\mathfrak{g}, \mathfrak{g}] \quad, \quad \mathfrak{g}^{(2)}=\left[\mathfrak{g}^{(1)}, \mathfrak{g}^{(1)}\right] \quad, \ldots, \quad \mathfrak{g}^{(i)}=\left[\mathfrak{g}^{(i-1)}, \mathfrak{g}^{(i-1)}\right]
$$

Dizemos que $\mathfrak{g}$ é solúvel se $\mathfrak{g}^{(n)}=\{0\}$, para algum $n$. Por exemplo, abeliana implica solúvel, enquanto que álgebras simples são definitivamente não solúveis.

Vejamos agora algumas observações sobre solubilidade:

Proposição A.2.1. Seja $\mathfrak{g}$ uma álgebra de Lie.

1. Se $\mathfrak{g}$ é solúvel, então todas as subálgebras e imagens homomórficas de $\mathfrak{g}$ são solúveis.

2. Se $\mathfrak{h}$ é um ideal solúvel de $\mathfrak{g}$ tal que $\mathfrak{g} / \mathfrak{h}$ é solúvel, então $\mathfrak{g}$ é solúvel. 
3. Se $\mathfrak{h}_{1}, \mathfrak{h}_{2}$ são ideais solúveis de $\mathfrak{g}$, então $\mathfrak{h}_{1}+\mathfrak{h}_{2}$ é solúvel.

Demonstração. [Hum13], Capítulo I, Seção 3.1, pág. 11.

Seja $\mathfrak{g}$ uma álgebra de Lie arbitrária e seja $\mathfrak{h}$ um ideal maximal solúvel (isto é, não incluso em nenhum ideal solúvel maior). Se $\mathfrak{h}^{\prime}$ é algum outro ideal solúvel de $\mathfrak{g}$, então, pela Proposição A.2.1(3) e pela maximalidade de $\mathfrak{h}, \mathfrak{h}+\mathfrak{h}^{\prime}=\mathfrak{h}$. Isto prova a existência de um único ideal maximal solúvel. Temos o seguinte resultado:

Proposição A.2.2. Seja $\mathfrak{g}$ uma álgebra de Lie de dimensão finita. Então, existe em $\mathfrak{g}$ um único ideal solúvel $\mathfrak{r}(\mathfrak{g}) \subseteq \mathfrak{g}$ que contém todos os ideais solúveis de $\mathfrak{g}$.

Demonstração. [Mar10], Capítulo 1, Proposição 1.28, pág. 49.

O ideal da proposição anterior é chamado de radical solúvel de $\mathfrak{g}$ e é denotado por $\mathfrak{r}(\mathfrak{g})$. No caso em que o $\mathfrak{r}(\mathfrak{g})=\{0\}, \mathfrak{g}$ é chamada semissimples. Uma álgebra de Lie semissimples é uma soma direta de álgebras de Lie simples.

A definição de solubilidade imita a correspondente noção em teoria dos grupos, dadas por Abel e Galois. Em contraste, a noção de grupo nilpotente é mais recente, e existe uma noção correspondente para álgebras de Lie. Definimos a sequência de ideias de $\mathfrak{g}$, a série central descendente, por:

$$
\mathfrak{g}^{0}=\mathfrak{g} \quad, \quad \mathfrak{g}^{1}=[\mathfrak{g}, \mathfrak{g}]\left(=\mathfrak{g}^{(1)}\right) \quad, \quad \mathfrak{g}^{2}=\left[\mathfrak{g}, \mathfrak{g}^{1}\right] \quad, \ldots, \quad \mathfrak{g}^{i}=\left[\mathfrak{g}, \mathfrak{g}^{i-1}\right]
$$

Dizemos que $\mathfrak{g}$ é nilpotente se $\mathfrak{g}^{n}=\{0\}$, para algum $n$. Por exemplo, qualquer álgebra abeliana é nilpotente. Claramente, $\mathfrak{g}^{(i)} \subseteq \mathfrak{g}^{i}$ para todo $i$, então álgebras nilpotentes são solúveis. A recíproca é falsa, entretanto.

Vejamos agora algumas propriedades sobre nilpotência:

Proposição A.2.3. Seja $\mathfrak{g}$ uma álgebra de Lie.

1. Se $\mathfrak{g}$ é nilpotente, então todas as subálgebras e imagens homomórficas de $\mathfrak{g}$ são nilpotentes.

2. Se $\mathfrak{g} / Z(\mathfrak{g})$ é nilpotente, então $\mathfrak{g}$ é nilpotente.

3. Se $\mathfrak{g}$ é nilpotente e não nula, então $Z(\mathfrak{g}) \neq\{0\}$.

Demonstração. [Hum13], Capítulo I, Seção 3.2, pág. 12.

A condição para $\mathfrak{g}$ ser nilpotente pode ser reescrita como segue: para algum $n$ (dependendo somente de $\mathfrak{g}), \operatorname{ad}\left(x_{1}\right) \operatorname{ad}\left(x_{2}\right) \cdots a d\left(x_{n}\right)(y)=0$, para todos $x_{i}, y \in \mathfrak{g}$. Em particular, $(a d(x))^{n}=0$ para todos $x \in \mathfrak{g}$. Agora se $\mathfrak{g}$ é qualquer álgebra de Lie, e $x \in \mathfrak{g}$, dizemos que $x$ é ad-nilpotente se ad(x) é um endomorfismo nilpotente. Usando esta linguagem: se $\mathfrak{g}$ é nilpotente, então todos os elementos de $\mathfrak{g}$ são $a d$-nilpotentes. A recíproca também é verdadeira.

Teorema A.2.2 (Teorema de Engel). Se todos os elementos de $\mathfrak{g}$ são ad-nilpotentes, então $\mathfrak{g}$ é nilpotente.

Demonstração. [Hum13], Capítulo I, Seção 3.3, pág. 13.

Lema A.2.1. Seja $x \in \mathfrak{g l}(V)$ um endomorfismo nilpotente. Então ad(x) é nilpotente.

Demonstração. [Hum13], Capítulo I, Seção 3.2, pág. 12. 
O Teorema de Engel A.2.2 é deduzido do seguinte resultado:

Teorema A.2.3. Seja $\mathfrak{g}$ uma subálgebra de $\mathfrak{g} \mathfrak{l}(V), V$ com dimensão finita. Se $\mathfrak{g}$ consiste de endomorfismos nilpotentes e $V \neq\{0\}$, então existe $v \in V$ não nulo para o qual $\mathfrak{g} \cdot v=0$.

Demonstração. [Hum13], Capítulo I, Seção 3.3, pág. 13.

\section{A.2.5 Teoremas de Lie e Cartan}

Com o intuito de ter disponíveis os autovalores de $a d(x)$ para $x$ arbitrário (não somente para $a d(x)$ nilpotente), vamos assumir que $k$ é um corpo algebricamente fechado.

A essência do Teorema de Engel para álgebras de Lie nilpotentes é a existência de um autovetor comum para uma álgebra de Lie consistindo de endomorfismos nilpotentes (Teorema A.2.3). O próximo Teorema é similar em natureza, mas requer o fecho algébrico do corpo $k$, a fim de assegurar que $k$ conterá todos os autovalores necessários.

Teorema A.2.4. Seja $\mathfrak{g}$ uma subálgebra solúvel de $\mathfrak{g l}(V), V$ com dimensão finita. Se $V \neq$ $\{0\}$, então $V$ contém um autovetor comum para todos os endomorfismos em $\mathfrak{g}$.

Demonstração. [Hum13], Capítulo II, Seção 4.1, pág. 15.

Corolário A.2.1 (Teorema de Lie). Seja $\mathfrak{g}$ uma subálgebra solúvel de $\mathfrak{g l}(V)$, $\operatorname{dim}(V)=$ $n<\infty$. Então as matrizes de $\mathfrak{g}$ em relaçãa à uma base adequada de $V$ são triangulares superiores.

Demonstração. [Hum13], Capítulo II, Seção 4.1, pág. 16.

Corolário A.2.2. Seja $\mathfrak{g}$ uma álgebra de Lie solúvel. Então existe uma cadeia de ideias de $\mathfrak{g}$,

$$
\{0\}=\mathfrak{g}_{0} \subseteq \mathfrak{g}_{1} \subseteq \ldots \subseteq \mathfrak{g}_{n}=\mathfrak{g}
$$

tal que $\operatorname{dim}\left(\mathfrak{g}_{i}\right)=i$.

Demonstração. [Hum13], Capítulo II, Seção 4.1, pág. 16.

Corolário A.2.3. Seja $\mathfrak{g}$ uma álgebra de Lie solúvel. Então $x \in[\mathfrak{g}, \mathfrak{g}]$ implica que ad $d_{\mathfrak{g}}(x)$ é nilpotente. Em particular, $[\mathfrak{g}, \mathfrak{g}]$ é nilpotente.

Demonstração. [Hum13], Capítulo II, Seção 4.1, pág. 17.

Temos que em uma álgebra de Lie de dimensão finita existe um ideal nilpotente que engloba todos os ideais nilpotentes:

Proposição A.2.4. Seja $\mathfrak{g}$ uma álgebra de Lie de dimensão finita. Então, existe um ideal de $\mathfrak{g}$, denotado por $\mathfrak{n} \mathfrak{n}(\mathfrak{g})$ e denominado radical nilpotente ou nil-radical de $\mathfrak{g}$, que contém todo ideal nilpotente de $\mathfrak{g}$.

Demonstração. [Mar10], Capítulo 2, Proposição 2.17, pág. 74.

Agora vamos obter um critério poderoso para solubilidade de uma álgebra de Lie $\mathfrak{g}$, baseado nos traços de certos endomorfismos de $\mathfrak{g}$. É óbvio que $\mathfrak{g}$ será solúvel se $[\mathfrak{g}, \mathfrak{g}]$ é nilpotente (esta é a recíproca do Corolário A.2.3). Por sua vez, o Teorema de Engel A.2.2 $\operatorname{diz}$ que $[\mathfrak{g}, \mathfrak{g}]$ será nilpotente se (e somente se) cada $a d_{[\mathfrak{g}, \mathfrak{g}]}(x), x \in[\mathfrak{g}, \mathfrak{g}]$, é nilpotente. 
Lema A.2.2. Sejam $A \subseteq B$ dois subespaços de $\mathfrak{g l}(V), \operatorname{dim}(V)<\infty$. Seja $M=\{x \in$ $\mathfrak{g l}(V) \mid[x, B] \subseteq A\}$. Suponha que $x \in M$ satisfaz $\operatorname{tr}(x y)=0$, para todos $y \in M$. Entãa $x$ é nilpotente.

Demonstração. [Hum13], Capítulo II, Seção 4.3, pág. 19.

Teorema A.2.5 (Critério de Cartan). Seja $\mathfrak{g}$ uma subálgebra de $\mathfrak{g l}(V), V$ com dimensão finita. Suponha que $\operatorname{tr}(x y)=0$, para todos $x \in[\mathfrak{g}, \mathfrak{g}], y \in \mathfrak{g}$. Então $\mathfrak{g}$ é solúvel.

Demonstração. [Hum13], Capítulo II, Seção 4.3, pág. 20.

Corolário A.2.4. Seja $\mathfrak{g}$ uma álgebra de Lie tal que $\operatorname{tr}(\operatorname{ad}(x) \operatorname{ad}(y))=0$, para todos $x \in$ $[\mathfrak{g}, \mathfrak{g}], y \in \mathfrak{g}$. Então $\mathfrak{g}$ é solúvel.

Demonstração. [Hum13], Capítulo II, Seção 4.3, pág. 20.

Seja $\mathfrak{g}$ uma álgebra de Lie qualquer. Se $x, y \in \mathfrak{g}$, definimos $\kappa(x, y)=\operatorname{tr}(\operatorname{ad}(x) \operatorname{ad}(y))$. Então $\kappa$ é uma forma bilinear simétrica em $\mathfrak{g}$, chamada de forma de Killing. $\kappa$ é também associativa, no sentido que $\kappa([x, y], z)=\kappa(x,[y, z])$. Isto segue da identidade $\operatorname{tr}([x, y] z)=\operatorname{tr}(x[y, z])$, para endomorfismos $x, y, z$ de um espaço vetorial de dimensão finita.

Lema A.2.3. Seja $\mathfrak{h}$ um ideal de $\mathfrak{g}$. Se $\kappa$ é a forma de Killing de $\mathfrak{g}$ e $\kappa_{\mathfrak{h}}$ a forma de Killing de $\mathfrak{h}$ (vistos como álgebras de Lie), então $\kappa_{\mathfrak{h}}=\left.\kappa\right|_{\mathfrak{h} \times \mathfrak{h}}$.

Demonstração. [Hum13], Capítulo II, Seção 5.1, pág. 21.

Em geral, uma forma bilinear simétrica $\beta(x, y)$ é chamada de não degenerada se seu radical $S$ é $\{0\}$, em que $S=\{x \in \mathfrak{g} \mid \beta(x, y)=0$, para todos $y \in \mathfrak{g}\}$. Como a forma de Killing é associativa, seu radical é mais do que um subespaço: $S$ é um ideal de g. Da álgebra linear, uma maneira prática para testar a não degeneralidade é como segue: fixemos uma base $x_{1}, \ldots, x_{n}$ de $\mathfrak{g}$. Então $\kappa$ é não degenerada se, e somente se, a matriz $n \times n$ cuja entrada $(i, j)$ é $\kappa\left(x_{i}, x_{j}\right)$ tem determinante não nulo.

Teorema A.2.6. Seja $\mathfrak{g}$ uma álgebra de Lie. Então $\mathfrak{g}$ é semissimples se, e somente se, sua forma de Killing é não degenerada.

Demonstração. [Hum13], Capítulo II, Seção 5.1, pág. 22.

Vamos enunciar uma importante consequência da não degeneralidade da forma de Killing. Antes observamos que $a d(\mathfrak{g})$ é um ideal de $\operatorname{Der}_{k}(\mathfrak{g}$ ) (para qualquer álgebra de Lie $\mathfrak{g}$ ), pois $[\delta, \operatorname{ad}(x)]=a d(\delta x)$, para todos $x \in \mathfrak{g}, \delta \in \operatorname{Der}_{k}(\mathfrak{g})$.

Teorema A.2.7. Se $\mathfrak{g}$ é uma álgebra de Lie semissimples, então ad( $\mathfrak{g})=\operatorname{Der}_{k}(\mathfrak{g})$ (isto é, toda derivação de $\mathfrak{g}$ é interna).

Demonstração. [Hum13], Capítulo II, Seção 5.3, pág. 23.

O Teorema A.2.7 pode ser usado para introduzir uma decomposição de Jordan abstrata em uma álgebra de Lie g semissimples arbitrária.

Antes vamos considerar alguns fatos. Sejam $V$ um espaço vetorial sobre $k$ de dimensão finita e $x \in \operatorname{End}_{k}(V)$. Chamamos $x$ de semissimples se as raízes do seu polinômio minimal sobre $k$ são todas distintas. Equivalentemente, $x$ é semissimples se, e somente se, $x$ é diagonalizável. Observamos que dois endomorfismos semissimples que comutam entre si, podem ser simultaneamente diagonalizados. A decomposição $x=x_{s}+x_{n}$ é chamada de decomposiçãa de Jordan-Chevalley (aditiva) de $x$, ou simplesmente decomposiçãa de Jordan; 
$x_{s}, x_{n}$ são chamadas, respectivamente, a parte semissimples e a parte nilpotente de $x$. Então $\operatorname{ad}(x)=a d\left(x_{s}\right)+a d\left(x_{n}\right)$ é a decomposição de Jordan de $\operatorname{ad}(x)$ em $\operatorname{End}\left(\operatorname{End}_{k}(V)\right)$ (ver [Hum13], Capítulo II, Seção 4.2, Lema $A$ ).

Se $A$ é alguma $k$-álgebra de dimensão finita, então $\operatorname{Der}_{k}(A)$ contém as partes semissimples e nilpotentes de todos os seus elementos (ver [Hum13], Capítulo $I I$, Seção 4.2, Lema $B$ ).

Em particular, como $\operatorname{Der}_{k}(\mathfrak{g})$ coincide com $\operatorname{ad}(\mathfrak{g})$ (Teorema A.2.7), enquanto $\mathfrak{g} \rightarrow \operatorname{ad}(\mathfrak{g})$ é $1-1$, cada $x \in \mathfrak{g}$ determina únicos elementos $s, n \in \mathfrak{g}$ tal que $\operatorname{ad}(x)=\operatorname{ad}(s)+\operatorname{ad}(n)$ é a decomposição de Jordan de $a d(x)$ em $\operatorname{End}_{k}(\mathfrak{g})$.

O Critério de Cartan (Teorema A.2.5) para solubilidade é usado para provar que uma álgebra de Lie $\mathfrak{g}$ semissimples tem forma de Killing não degenerada. Em geral, seja $\mathfrak{g}$ uma álgebra de Lie semissimples e seja $\phi: \mathfrak{g} \rightarrow \mathfrak{g l}(V)$ uma representação fiel de $\mathfrak{g}$. Definimos uma forma bilinear simétrica $\beta(x, y)=\operatorname{tr}(\phi(x) \phi(y))$ em $\mathfrak{g}$. A forma $\beta$ é associativa (em particular seu radical é um ideal). Além disso, $\beta$ é não degenerada: de fato, o Teorema A.2.5 mostra que $\phi(S) \cong S$ é solúvel, então $S=\{0\}$ (a forma de Killing é simplesmente $\beta$ no caso especial $\phi=a d)$.

Agora, sejam $\mathfrak{g}$ uma álgebra de Lie semissimples e $\beta$ qualquer forma bilinear associativa simétrica não degenerada em $\mathfrak{g}$. Se $\left\{x_{1}, \ldots, x_{n}\right\}$ é uma base de $\mathfrak{g}$, existe uma única base dual $\left\{y_{1}, \ldots, y_{n}\right\}$ relativa a $\beta$, satisfazendo $\beta\left(x_{i}, y_{j}\right)=\delta_{i j}$. Se $\phi: \mathfrak{g} \rightarrow \mathfrak{g l}(V)$ é qualquer representação de $\mathfrak{g}$, tomemos $c_{\phi}(\beta)=\sum_{i} \phi\left(x_{i}\right) \phi\left(y_{i}\right) \in \operatorname{End}_{k}(V)\left(x_{i}, y_{i}\right.$ percorrendo a base dual de $\beta) . c_{\phi}(\beta)$ é um endomorfismo de $V$ que comuta com $\phi(\mathfrak{g})$.

No caso que $\phi: \mathfrak{g} \rightarrow \mathfrak{g l}(V)$ é uma representação fiel, com forma traço (não degenerada) $\beta(x, y)=\operatorname{tr}(\phi(x) \phi(y))$, fixando uma base $\left\{x_{1}, \ldots, x_{n}\right\}$ de $\mathfrak{g}$, escrevemos simplesmente $c_{\phi}$ para $c_{\phi}(\beta)$ e o chamamos de elemento Casimir de $\phi$.

\section{A.2.6 Representações de $\mathfrak{s l}(2, \boldsymbol{k})$}

Nesta Subseção vamos considerar que todos os módulos possuem dimensão finita sobre $k$, em que $k$ é um corpo algebricamente fechado. Seja $\mathfrak{g}=\mathfrak{s l}(2, k)$, cuja a base canônica consiste de:

$$
x=\left(\begin{array}{ll}
0 & 1 \\
0 & 0
\end{array}\right) \quad, \quad y=\left(\begin{array}{ll}
0 & 0 \\
1 & 0
\end{array}\right) \quad \text { e } \quad h=\left(\begin{array}{cc}
1 & 0 \\
0 & -1
\end{array}\right)
$$

em que a tábua de multiplicação é completamente determinada pelas equações:

$$
[x, y]=h \quad, \quad[h, x]=2 x \quad e \quad[h, y]=-2 y
$$

Seja $V$ um g-módulo arbitrário. Como $h$ é semissimples, implica que $h$ age diagonalmente em $V$. Como $k$ é algebricamente fechado, todos os autovalores desejados estão em $k$. Podemos considerar uma decomposição de $V$ como soma direta de autoespaços $V_{\lambda}=\{v \in V \mid h \cdot v=$ $\lambda \cdot v\}, \lambda \in k$. Claramente, o subespaço $V_{\lambda}$ é $\{0\}$ quando $\lambda$ não é autovalor para o endomorfismo de $V$ o qual representa $h$. Sempre que, $V_{\lambda} \neq\{0\}$, dizemos que $\lambda$ é um peso de $h$ em $V$ e dizemos que $V_{\lambda}$ é um espaço de peso.

Lema A.2.4. Se $v \in V_{\lambda}$, então $x \cdot v \in V_{\lambda+2}$ e $y \cdot v \in V_{\lambda-2}$.

Demonstração. [Hum13], Capítulo II, Seção 7.1, pág. 32.

Como $\operatorname{dim}(V)<\infty$, e a soma $V=\bigoplus_{\lambda \in k} V_{\lambda}$ é direta, deve existir $V_{\lambda} \neq\{0\}$ tal que $V_{\lambda+2}=\{0\}$ (graças ao Lema A.2.4, $x \cdot v=0$, para algum $v \in V_{\lambda}$ ). Em geral, qualquer vetor não nulo em $V_{\lambda}$ aniquilado por $x$ será chamado de vetor maximal de peso $\lambda$.

Vamos assumir agora que $V$ é um $\mathfrak{g}$-módulo irredutível. Escolhemos um vetor maximal $v_{0} \in V_{\lambda}$. Sejam $v_{-1}=0$ e $v_{i}=(1 / i !) y^{i} \cdot v_{0}(i \geq 0)$. 
Lema A.2.5. 1. $h \cdot v_{i}=(\lambda-2 i) v_{i}$,

2. $y \cdot v_{i}=(i+1) v_{i+1}$,

3. $x \cdot v_{i}=(\lambda-i+1) v_{i-1} \quad(i \geq 0)$.

Demonstração. [Hum13], Capítulo II, Seção 7.2, pág. 32.

Por causa do Lema A.2.5(1), os vetores $v_{i}$ não nulos são todos linearmente independentes. Como a $\operatorname{dim}(V)<\infty$, seja $m$ o menor inteiro para o qual $v_{m} \neq 0$ e $v_{m+1}=0$; evidentemente $v_{m+i}=0$, para todo $i>0$. Os itens (1)-(3) do Lema A.2.5 juntos, mostram que o subespaço de $V$ com base $\left\{v_{0}, v_{1}, \ldots, v_{m}\right\}$ é um $\mathfrak{g}$-submódulo, diferente de $\{0\}$. Como $V$ é irredutível, o subespaço deve ser o espaço $V$. Além disso, em relação à base ordenada $\left\{v_{0}, v_{1}, \ldots, v_{m}\right\}$, as matrizes dos endomorfismos representando $x, y, h$, são uma matriz triangular superior nilpotente, uma matriz triangular inferior nilpotente e uma matriz diagonal, respectivamente.

Um olhar mais atento ao Lema A.2.5, item 3, revela um fato: para $i=m+1$, o lado esquerdo é zero, enquanto que o lado direito é $(\lambda-m) v_{m}$. Como $v_{m} \neq 0$, concluímos que $\lambda=$ $m$. Em outras palavras, o peso de um vetor maximal é um inteiro não negativo $(\operatorname{dim}(V)-1)$. Dizemos que ele é o peso máximo de $V$. Além disso, cada peso $\mu$ ocorre com multiplicidade um (isto é, $\operatorname{dim}\left(V_{\mu}\right)=1$, se $V_{\mu} \neq\{0\}$ ). Em particular, como $V$ determina $\lambda$ unicamente $(\lambda=\operatorname{dim}(V)-1)$, o vetor maximal $v_{0}$ é o único possível em $V$ (além dos múltiplos escalares não nulos). Para resumir:

Teorema A.2.8. Seja $V$ um módulo irredutivel sobre $\mathfrak{g}=\mathfrak{s l}(2, k)$.

1. Em relação à $h, V$ é a soma direta de espaços de peso $V_{\mu}$, com $\mu=m, m-2, \ldots,-(m-$ $2),-m$, em que $m+1=\operatorname{dim}(V)$ e $\operatorname{dim}\left(V_{\mu}\right)=1$, para cada $\mu$.

2. V tem (a menos de múltiplos escalares) um único vetor maximal, cujo peso (chamado de peso máximo de $V$ ) é $m$.

3. A ação de $\mathfrak{g}$ em $V$ é dada explicitamente pelo Lema A.2.5, se a base é escolhida na forma prescrita. Em particular, existe no máximo um $\mathfrak{g}$-módulo irredutível (a menos de isomorfismo) de cada dimensão possivel $m+1, m \geq 0$.

\section{A.3 Teoria de estrutura}

\section{A.3.1 Decomposição de Cartan}

Seja $\mathfrak{g}$ uma álgebra de Lie semissimples não nula. Vamos estudar a estrutura de $\mathfrak{g}$, via sua representação adjunta.

Se $\mathfrak{g}$ consiste inteiramente de elementos nilpotentes (isto é, ad-nilpotentes), então $\mathfrak{g}$ deve ser nilpotente (Teorema de Engel A.2.2). Se este não for o caso, podemos encontrar $x \in \mathfrak{g}$ cuja parte semissimples $x_{s}$ na decomposição abstrata de Jordan é não nula. Isso mostra que $\mathfrak{g}$ possui subálgebras não nulas (geradas pelos $x_{s}$ ) consistindo de elementos semissimples. Chamamos cada uma dessas subálgebras de subálgebra toral.

Lema A.3.1. Uma subálgebra toral de $\mathfrak{g}$ é abeliana.

Demonstração. [Hum13], Capítulo II, Seção 8.1, pág. 35. 
Agora fixemos uma subálgebra toral maximal $\mathfrak{h}$ de $\mathfrak{g}$, isto é, uma subálgebra toral que não está propriamente contida em nenhuma outra. Por exemplo, se $\mathfrak{g}=\mathfrak{s l}(n, k)$, então ho pode ser tomada como o conjunto das matrizes diagonais (de traço 0 ).

Como $\mathfrak{h}$ é abeliana (pelo Lema A.3.1), $a d_{\mathfrak{g}}(\mathfrak{h})$ é uma família comutativa de endomorfismos semissimples de $\mathfrak{g}$. De acordo com um resultado de Álgebra Linear, $a d_{\mathfrak{g}}(\mathfrak{h})$ é simultaneamente diagonalizável. Em outras palavras, $\mathfrak{g}$ é uma soma direta dos subespaços $\mathfrak{g}_{\alpha}=\{x \in \mathfrak{g} \mid[h, x]=\alpha(h) x$, para todos $h \in \mathfrak{h}\}$, em que $\alpha$ percorre $\mathfrak{h}^{*}$. Notemos que $\mathfrak{g}_{0}$ é simplesmente $C_{\mathfrak{g}}(\mathfrak{h})$, o centralizador de $\mathfrak{h}$; ele contém $\mathfrak{h}$ por conta do Lema A.3.1. O conjunto de todos $\alpha \in \mathfrak{h}^{*}$ não nulos para os quais $\mathfrak{g}_{\alpha} \neq\{0\}$ é denotado por $\Phi$; os elementos de $\Phi$ são chamados de raízes de $\mathfrak{g}$ em relação a h (e são uma quantidade finita). Com esta notação temos um decomposição em espaços de raízes (ou decomposição de Cartan): $\mathfrak{g}=C_{\mathfrak{g}}(\mathfrak{h}) \bigoplus\left(\bigoplus_{\alpha \in \Phi} \mathfrak{g}_{\alpha}\right)$

Proposição A.3.1. Para todos $\alpha, \beta \in \mathfrak{h}^{*},\left[\mathfrak{g}_{\alpha}, \mathfrak{g}_{\beta}\right] \subseteq \mathfrak{g}_{\alpha+\beta}$. Se $x \in \mathfrak{g}_{\alpha}$, com $\alpha \neq 0$, então $a d(x)$ é nilpotente. Se $\alpha, \beta \in \mathfrak{h}^{*}$, e $\alpha+\beta \neq 0$, então $\mathfrak{g}_{\alpha}$ é ortogonal à $\mathfrak{g}_{\beta}$, com relação a forma de Killing $\kappa$ de $\mathfrak{g}$.

Demonstração. [Hum13], Capítulo II, Seção 8.1, pág. 36.

Corolário A.3.1. A restrição da forma de Killing para $\mathfrak{g}_{0}=C_{\mathfrak{g}}(\mathfrak{h})$ é não degenerada.

Demonstração. [Hum13], Capítulo II, Seção 8.1, pág. 36.

Proposição A.3.2. Seja $\mathfrak{h}$ uma subálgebra toral maximal de $\mathfrak{g}$. Então $\mathfrak{h}=C_{\mathfrak{g}}(\mathfrak{h})$.

Demonstração. [Hum13], Capítulo II, Seção 8.2, pág. 36.

Corolário A.3.2. A restrição da forma de Killing $\kappa$ para h é não degenerada.

Demonstração. [Hum13], Capítulo II, Seção 8.2, pág. 37.

O corolário identifica $\mathfrak{h}$ com $\mathfrak{h}^{*}$ : cada $\phi \in \mathfrak{h}^{*}$ corresponde ao (único) elemento $t_{\phi} \in \mathfrak{h}$, satisfazendo $\phi(h)=\kappa\left(t_{\phi}, h\right)$ para todo $h \in \mathfrak{h}$. Em particular, $\Phi$ corresponde ao subconjunto $\left\{t_{\alpha} \mid \alpha \in \Phi\right\}$ de $\mathfrak{h}$.

Pela Proposição A.3.1 se $\alpha, \beta \in \mathfrak{h}^{*}$ e $\alpha+\beta \neq 0$ temos que $\kappa\left(\mathfrak{g}_{\alpha}, \mathfrak{g}_{\beta}\right)=0$; em particular, $\kappa\left(\mathfrak{h}, \mathfrak{g}_{\alpha}\right)=0$, para todo $\alpha \in \Phi$ (Proposição A.3.2), então a restrição de $\kappa$ para $\mathfrak{h}$ é não degenerada.

\section{Proposição A.3.3.}

1. $\Phi$ gera $\mathfrak{h}^{*}$.

2. Se $\alpha \in \Phi$, então $-\alpha \in \Phi$.

3. Sejam $\alpha \in \Phi, x \in \mathfrak{g}_{\alpha}, y \in \mathfrak{g}_{-\alpha}$. Então $[x, y]=\kappa(x, y) t_{\alpha}$.

4. Se $\alpha \in \Phi$, então $\left[\mathfrak{g}_{\alpha}, \mathfrak{g}_{-\alpha}\right]$ é unidimensional, com base $t_{\alpha}$.

5. $\alpha\left(t_{\alpha}\right)=\kappa\left(t_{\alpha}, t_{\alpha}\right) \neq 0$, para todos $\alpha \in \Phi$.

6. Se $\alpha \in \Phi$ e $x_{\alpha}$ é algum elemento não nulo de $\mathfrak{g}_{\alpha}$, então existe $y_{\alpha} \in \mathfrak{g}_{-\alpha}$ tais que $x_{\alpha}, y_{\alpha}, h_{\alpha}=\left[x_{\alpha}, y_{\alpha}\right]$, geram uma subálgebra de $\mathfrak{g}$ simples tridimensional isomorfa à $\mathfrak{s l}(2, k)$ via:

$$
x_{\alpha} \mapsto\left(\begin{array}{cc}
0 & 1 \\
0 & 0
\end{array}\right) \quad, \quad y_{\alpha} \mapsto\left(\begin{array}{cc}
0 & 0 \\
1 & 0
\end{array}\right) \quad, \quad h_{\alpha} \mapsto\left(\begin{array}{cc}
1 & 0 \\
0 & -1
\end{array}\right)
$$


7. $h_{\alpha}=\frac{2 t_{\alpha}}{\kappa\left(t_{\alpha}, t_{\alpha}\right)} ; h_{\alpha}=-h_{-\alpha}$.

Demonstração. [Hum13], Capítulo II, Seção 8.3, pág. 37.

\section{A.3.2 Sistema de raízes}

Vamos fixar um espaço euclideano $E$, isto é, um espaço vetorial sobre $\mathbb{R}$ de dimensão finita munido com uma forma bilinear simétrica positiva definida $(\alpha, \beta)$. Geometricamente, uma reflexão em $E$ é uma transformação linear inversível que deixa fixo algum hiperplano (subespaço de codimensão um) e envia qualquer vetor ortogonal ao hiperplano em seu negativo. Qualquer vetor $\alpha$ determina uma reflexão $\sigma_{\alpha}$, com hiperplano refletido $P_{\alpha}=\{\beta \in E \mid(\beta, \alpha)=0\}$. Temos que:

$$
\sigma_{\alpha}(\beta)=\beta-\frac{2(\beta, \alpha)}{(\alpha, \alpha)} \alpha
$$

Como o número $2(\beta, \alpha) /(\alpha, \alpha)$ ocorre frequentemente, abreviaremos ele por $\langle\beta, \alpha\rangle$. Notemos que $\langle\beta, \alpha\rangle$ é linear somente na primeira variável.

Um subconjunto $\Phi$ do espaço euclideano $E$ é chamado um sistema de raízes em $E$ se os seguintes axiomas são satisfeitos:

(R1) $\Phi$ é finito, gera $E$, e não contém o 0 (o vetor nulo).

(R2) Se $\alpha \in \Phi$, os únicos múltiplos de $\alpha$ em $\Phi$ são $\pm \alpha$.

(R3) Se $\alpha \in \Phi$, a reflexão $\sigma_{\alpha}$ deixa $\Phi$ invariante.

(R4) Se $\alpha, \beta \in \Phi$, então $\langle\beta, \alpha\rangle \in \mathbb{Z}$.

Chamaremos $l=\operatorname{dim}(E)$ de posto do sistema de raízes $\Phi$.

$\Phi$ é chamado irredutivel se não pode ser particionado em uma união de dois subconjuntos próprios tais que cada raiz em um conjunto é ortogonal a cada raiz do outro.

Seja $\Phi$ um sistema de raízes em $E$. Denotemos por $\mathbb{W}$ o subgrupo de $G L(E)$ gerado pelas reflexões $\sigma_{\alpha}$, com $\alpha \in \Phi$. Por (R3), W permuta o conjunto $\Phi$, que por (R1) é finito e gera $E$. Podemos identificar $\mathbb{W}$ com um subgrupo do grupo simétrico de $\Phi$; em particular, $\mathbb{W}$ é finito. $\mathbb{W}$ é chamado de grupo de Weyl de $\Phi$.

Existe a noção de isomorfismo entre sistemas de raízes $\Phi, \Phi^{\prime}$ dos respectivos espaços euclideanos $E, E^{\prime}$ : dizemos que $(\Phi, E)$ e $\left(\Phi^{\prime}, E^{\prime}\right)$ são isomorfos se existe um isomorfismo de espaços vetoriais (não necessariamente uma isometria) $\phi: E \rightarrow E^{\prime}$ enviando $\Phi$ em $\Phi^{\prime}$ tal que $\langle\phi(\beta), \phi(\alpha)\rangle=\langle\beta, \alpha\rangle$, para cada $\operatorname{par} \alpha, \beta \in \Phi$.

Seja $\Phi$ um sistema de raízes com posto $l$ em um espaço euclideano $E$, com grupo de Weyl $\mathbb{W}$. Um subconjunto $\Delta$ de $\Phi$ é chamado de base se:

(B1) $\Delta$ é uma base de $E$,

(B2) Toda raiz $\beta$ pode ser escrita como $\beta=\sum k_{\alpha} \alpha \quad(\alpha \in \Delta)$ com coeficientes inteiros $k_{\alpha}$, todos não negativos ou não positivos para cada $\beta$.

Em vista de (B1) a cardinalidade de $\Delta$ é $l$, e a expressão para $\beta$ em (B2) é única. Definimos a altura de uma raiz $\beta=\sum k_{\alpha} \alpha$ (em relação à $\Delta$ ) por $h t(\beta)=\sum_{\alpha \in \Delta} k_{\alpha}$. Se todos os $k_{\alpha} \geq 0$ (resp. todos $k_{\alpha} \leq 0$ ), dizemos que $\beta$ é positiva (resp. negativa). 
As coleções das raízes positivas e negativas (em relação à $\Delta$ ) são usualmente denotadas por $\Delta^{+}$e $\Delta^{-}$. As raízes em $\Delta$ são chamadas raízes simples; fixemos uma ordem nestas raízes, digamos $\Delta=\left\{\alpha_{1}, \ldots, \alpha_{l}\right\}$, em que $l$ é a dimensão de $\mathfrak{h}$. Os elementos $\lambda_{i} \in \mathfrak{h}^{*}$ definidos como $\left\langle\lambda_{i}, \alpha_{j}\right\rangle=\delta_{i j}$ são chamados de pesos fundamentais com relação a $\Delta$. A matriz $\left(\left\langle\alpha_{i}, \alpha_{j}\right\rangle\right)_{i, j}$, a menos de permutações dos índices, é um invariante de $\mathfrak{g}$ e é chamada de Matriz de Cartan de $\mathfrak{g}$. As informações da Matriz de Cartan podem ser apresentadas no diagrama de Dynkin.

\section{A.3.3 Subálgebra de Cartan}

Definição A.3.1. Seja $\mathfrak{g}$ uma álgebra de Lie. Uma subálgebra de Cartan de $\mathfrak{g}$ é uma subálgebra $\mathfrak{h} \subseteq \mathfrak{g}$ que satisfaz

\section{1. h é nilpotente;}

2. O normalizador de $\mathfrak{h}$ em $\mathfrak{g}$ coincide com $\mathfrak{h}$, isto é, $N_{\mathfrak{g}}(\mathfrak{h})=\mathfrak{h}$.

Uma subálgebra de Cartan também é chamada de $C S A$. Esta definição não implica na existência das $C S A$ 's (na verdade, sobre corpos finitos a questão da existência não está totalmente resolvida). Para álgebras de Lie $\mathfrak{g}$ semissimples, sobre um corpo $k$ de característica 0 , as $C S A$ 's de $\mathfrak{g}$ são precisamente as subálgebras torais maximais de $\mathfrak{g}$. Para mais detalhes indicamos o Capítulo $I V$ de [Hum13].

Na sua tese em 1894, Élie Cartan classificou todas as álgebras de Lie simples de dimensão finita sobre $\mathbb{C}$. Estas álgebras de Lie são isomorfas as álgebras de Lie clássicas ou são isomorfas as chamadas álgebras de Lie excepcionais. Para um corpo $k$ algebricamente fechado com característica 0 , de acordo com os teoremas de classificação, existe uma (a menos de isomorfismo) e somente uma álgebra de Lie simples com sistema de raízes $A_{l}(l \geq 1)$, $B_{l}(l \geq 2), C_{l}(l \geq 3), D_{l}(l \geq 4), E_{6}, E_{7}, E_{8}, F_{4}, G_{2}$. Chamamos $E_{6}, E_{7}, E_{8}, F_{4}$ e $G_{2}$ de álgebras de Lie excepcionais, e um pré-requisito para estudá-las é a teoria das álgebras de Jordan. Para mais detalhes sobre a classificação das álgebras de Lie simples e sobre as álgebras de Lie excepcionais, indicamos [Hum13] (Capítulo V, Seção 19, pág. 102) e [Sam90] (Capítulo 2, Seções 13 e 14).

Esta classificação foi generalizada posteriormente para álgebras de Lie de dimensão finita sobre um corpo arbitrário de característica 0, principalmente por Claude Chevalley (19091984) e Harish-Chandra (1923-1983).

Em sua classificação, Élie Cartan introduziu o conceito de matrizes de Cartan, espaços de raízes e decomposição em espaços de raízes de uma álgebra de Lie.

\section{A.4 Álgebras Envolventes Universais}

Observamos que algumas definições e conceitos da teoria de categorias usados nessa Seção podem ser conferidos com mais detalhes no Apêndice B. Nessa Seção $k$ é um corpo arbitrário.

Vamos associar a cada álgebra de Lie $\mathfrak{g}$ sobre $k$ uma álgebra associativa com 1 (com dimensão infinita, em geral), que é gerada "livremente" por $\mathfrak{g}$ sujeita às relações de comutação em g. Esta é a álgebra envolvente universal que é uma ferramenta básica na teoria de representações.

Sejam $\mathfrak{A s s}_{k}$ a categoria de todas as álgebras associativas sobre um corpo $k$ e $\mathfrak{L i k}_{k}$ a categoria de todas as álgebras de Lie sobre $k$. Já definimos anteriormente $\mathfrak{A}^{(-)}$, como a 
álgebra de Lie obtida da álgebra associativa $\mathfrak{A}$. Como conjuntos, estas duas álgebras são iguais. Temos então a seguinte aplicação:

$$
\begin{aligned}
\mathfrak{g l}: \mathfrak{A l s s}_{k} & \longrightarrow \mathfrak{L} \mathfrak{i}_{k} \\
\mathfrak{A} & \longmapsto \mathfrak{g l}(\mathfrak{A})=\mathfrak{A}^{(-)}
\end{aligned}
$$

Proposição A.4.1. A aplicação gl é um funtor.

Demonstração. Já sabemos que $\mathfrak{g l}$ leva um $O b\left(\mathfrak{A}_{\mathfrak{s i}} \mathfrak{s}_{k}\right)$ em um $O b\left(\mathfrak{L i \mathfrak { k }}_{k}\right)$. Agora vamos mostrar que $\mathfrak{g l}$ leva um homomorfismo de $\mathfrak{A s s}_{k}$ em um homomorfismo de $\mathfrak{L i}_{k}$. De fato, sejam $\mathfrak{A}, \mathfrak{B} \in$ $\mathfrak{A s s}_{k}$ e $f: \mathfrak{A} \rightarrow \mathfrak{B}$ um homomorfismo de álgebras associativas. Então $f$ satisfaz as seguintes propriedades:

1. $f\left(1_{\mathfrak{A}}\right)=1_{\mathfrak{B}} ;$

2. $f(\lambda a)=\lambda f(a), \forall \lambda \in k e \forall a \in \mathfrak{A}$;

3. $f(a+b)=f(a)+f(b), \forall a, b \in \mathfrak{A}$;

4. $f(a b)=f(a) f(b), \forall a, b \in \mathfrak{A}$.

As propriedades 1., 2. e 3. dizem que $f$ é $k$-linear. Agora, utilizando 4., temos que:

$$
f([a, b])=f(a b-b a)=f(a b)-f(b a)=f(a) f(b)-f(b) f(a)=[f(a), f(b)], \forall a, b \in \mathfrak{A}
$$

Isso mostra que o homomorfismo $f$ de álgebras associativas pode ser estendido à um homomorfismo de álgebras de Lie. Note que a aplicação é a mesma. Sendo assim, denotaremos por $f$ um homomorfismo de álgebras associativas e por $f^{(-)}($ou $\mathfrak{g l}(f))$, o mesmo homomorfismo, visto como homomorfismo de álgebras de Lie. Temos então:

$$
f^{(-)}: \mathfrak{A}^{(-)} \rightarrow \mathfrak{B}^{(-)}
$$

Pelo diagrama abaixo podemos verificar que $g^{(-)} \circ f^{(-)}=(g \circ f)^{(-)}$, em que $\mathfrak{A}, \mathfrak{B}, \mathfrak{C} \in \mathfrak{A s s}_{k}$, e $f: \mathfrak{A} \rightarrow \mathfrak{B}$ e $g: \mathfrak{B} \rightarrow \mathfrak{C}$ são homomorfismos de álgebras associativas.

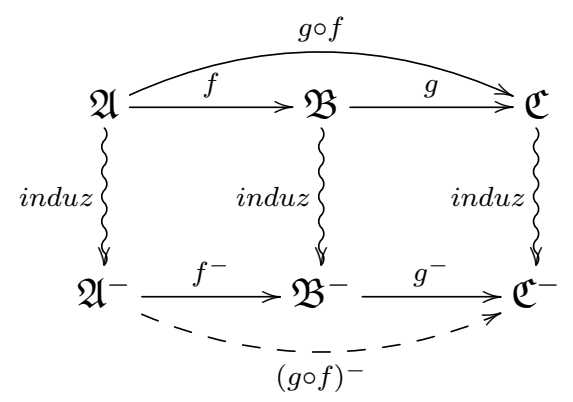

Portanto a aplicação $\mathfrak{g l}$ preserva a composição. Obviamente $i d_{\mathfrak{A}}: \mathfrak{A} \rightarrow \mathfrak{A}$, induz $\left(i d_{\mathfrak{A}}\right)^{(-)}$: $\mathfrak{A}^{(-)} \rightarrow \mathfrak{A}^{(-)}$. Portanto $\mathfrak{g l}$ é um funtor.

Agora vamos construir, partindo de uma álgebra de Lie $\mathfrak{g}$, uma álgebra associativa unital $U(\mathfrak{g})$.

Fixemos um espaço vetorial $V$ sobre $k$ de dimensão finita. Sejam $T^{0} V=k, T^{1} V=$ $V, T^{2} V=V \otimes V, \ldots, T^{m} V=V \otimes \ldots \otimes V$ ( $m$ cópias $)$. Definimos $\mathfrak{T}(V)=\bigoplus_{j=0}^{\infty} T^{j} V, \mathrm{e}$ introduzimos um produto associativo, definido nos geradores homogêneos de $\mathfrak{T}(V)$ pela regra 
$\left(v_{1} \otimes \ldots \otimes v_{k}\right) \cdot\left(w_{1} \otimes \ldots \otimes w_{m}\right)=v_{1} \otimes \ldots \otimes v_{k} \otimes w_{1} \otimes \ldots \otimes w_{m} \in T^{k+m} V$. Isto faz com que $\mathfrak{T}(V)$ seja uma álgebra graduada associativa com 1, que é gerada por 1 junto com qualquer base de $V$. Nós a chamamos de álgebra tensorial em $V . \mathfrak{T}(V)$ é a álgebra associativa universal em $n$ geradores $(n=\operatorname{dim}(V)$ ), no seguinte sentido: dado qualquer $k$-aplicação linear $\phi: V \rightarrow \mathfrak{A}$ ( $\mathfrak{A}$ uma álgebra associativa sobre $k$ com 1 ), existe um único homomorfismo de $k$-álgebras $\psi: \mathfrak{T}(V) \rightarrow \mathfrak{A}$ tal que $\psi(1)=1$ e o seguinte diagrama comuta ( $i$ é a inclusão):

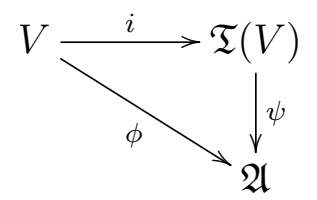

Agora seja $I$ um ideal bilateral em $\mathfrak{T}(V)$ gerado por todos $(x \otimes y)-(y \otimes x)(x, y \in$ $V$ ) e chamamos $\mathfrak{S}(V)=\mathfrak{T}(V) / I$ de álgebra simétrica em $V ; \sigma: \mathfrak{T}(V) \rightarrow \mathfrak{S}(V)$ denotará a aplicação canônica. Notemos que os geradores de $I$ encontram-se em $T^{2} V$. Então temos que $I=\left(I \cap T^{2} V\right) \bigoplus\left(I \cap T^{3} V\right) \bigoplus \ldots$ Portanto, $\sigma$ é injetivo em $T^{0} V=k, T^{1} V=V$ (o que nos permite identificar $V$ com um subespaço de $\mathfrak{S}(V)$ ), e $\mathfrak{S}(V)$ herda uma graduação de $\mathfrak{T}(V): \mathfrak{S}(V)=\bigoplus_{j=0}^{\infty} S^{j} V$. O efeito de fatorar $I$ é simplesmente fazer os elementos de $V$ comutarem. Então $\mathfrak{S}(V)$ é universal (no sentido acima) para aplicações lineares de $V$ em $k$-álgebras associativas comutativas com 1 . Além disso, se $\left\{x_{1}, \ldots, x_{n}\right\}$ é alguma base fixa de $V$, então $\mathfrak{S}(V)$ é canonicamente isomorfa à álgebra de polinômios sobre $k$ em $n$ variáveis, com base consistindo de 1 e todos $x_{i(1)} \cdots x_{i(t)}, t \geq 1,1 \leq i(1) \leq \ldots \leq i(t) \leq n$.

O grupo simétrico $\mathcal{S}_{m}$ age em $T^{m}(V)$ permutando os índices dos tensores $v_{1} \otimes \ldots \otimes v_{m}$ $\left(v_{i} \in V\right)$. Um elemento de $T^{m} V$ fixado por $\mathcal{S}_{m}$ é chamado de tensor simétrico homogêneo de ordem $m$.

Para uma álgebra de Lie $\mathfrak{g}$ arbitrária (que pode ter dimensão infinita), uma álgebra envolvente universal de $\mathfrak{g}$ é um par $(\mathfrak{U}, i)$, em que $\mathfrak{U}$ é uma álgebra associativa sobre $k$ com 1 e $i: \mathfrak{g} \rightarrow \mathfrak{U}$ é uma aplicação linear satisfazendo:

$$
i([x, y])=i(x) i(y)-i(y) i(x)
$$

para todos $x, y \in \mathfrak{g}$, e vale o seguinte: para qualquer $k$-álgebra associativa $\mathfrak{A}$ com 1 e qualquer aplicação linear $j: \mathfrak{g} \rightarrow \mathfrak{A}$ satisfazendo $j([x, y])=j(x) j(y)-j(y) j(x)$, existe um único homomorfismo de álgebras $\phi: \mathfrak{U} \rightarrow \mathfrak{A}$ (enviando 1 em 1 ) tal que $\phi \circ i=j$.

A unicidade de um tal par $(\mathfrak{U}, i)$ é de fácil verificação: dado um outro par $\left(\mathfrak{B}, i^{\prime}\right)$ satisfazendo as mesmas hipóteses, temos os homomorfismos $\phi: \mathfrak{U} \rightarrow \mathfrak{B}$ e $\psi: \mathfrak{B} \rightarrow \mathfrak{U}$. Por definição, existe uma única aplicação $\mathfrak{U} \rightarrow \mathfrak{U}$ que faz o seguinte diagrama comutar:

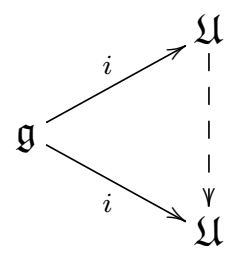

Mas $i d_{\mathfrak{U}}$ e $\psi \circ \phi$ satisfazem o diagrama, então $\psi \circ \phi=i d_{\mathfrak{U}}$. Similarmente, $\phi \circ \psi=i d_{\mathfrak{B}}$.

A existência do par $(\mathfrak{U}, i)$ pode ser vista em [Hum13], Capítulo $V$, Seção 17.2, pág. 91. Seja $\mathfrak{T}(\mathfrak{g})$ a álgebra tensorial em $\mathfrak{g}$, e seja $J$ o ideal bilateral em $\mathfrak{T}(\mathfrak{g})$ gerado por todos $(x \otimes y)-(y \otimes x)-[x, y] \quad(x, y \in \mathfrak{g})$. Definimos $U(\mathfrak{g})=\mathfrak{T}(\mathfrak{g}) / J$, e seja $\pi: \mathfrak{T}(\mathfrak{g}) \rightarrow U(\mathfrak{g})$ o homomorfismo canônico. Notemos que $J \subseteq \bigoplus_{j>0} T^{j} \mathfrak{g}$, então $\pi$ aplica $T^{0} \mathfrak{g}=k$ isomorficamente 
em $U(\mathfrak{g})$ (Portanto, $U(\mathfrak{g})$ contém os escalares). Afirmamos que $(U(\mathfrak{g}), i)$ é uma álgebra envolvente universal de $\mathfrak{g}$, em que $i: \mathfrak{g} \rightarrow U(\mathfrak{g})$ é a restrição de $\pi$ à $\mathfrak{g}$.

Até agora conhecemos pouco sobre a estrutura de $U(\mathfrak{g})$, exceto que ela contém os escalares.

Por simplicidade seja $\mathfrak{T}=\mathfrak{T}(\mathfrak{g}), \mathfrak{S}=\mathfrak{S}(\mathfrak{g})$ e $U=U(\mathfrak{g})$; Similarmente, escreveremos $T^{m}, S^{m}$.

Definimos uma filtração em $\mathfrak{T}$ por $T_{m}=T^{0} \bigoplus T^{1} \bigoplus \ldots \bigoplus T^{m}$, e seja $U_{m}=\pi\left(T_{m}\right), U_{-1}=$ $\{0\}$. Claramente, $U_{m} U_{p} \subseteq U_{m+p}$ e $U_{m} \subseteq U_{m+1}$. Seja $G^{m}=U_{m} / U_{m-1}$ (isto é simplesmente um espaço vetorial), e pela multiplicação em $U$ definimos uma aplicação bilinear $G^{m} \times G^{p} \rightarrow$ $G^{m+p}$ (a aplicação está bem definida). Esta se estende a uma aplicação bilinear $\mathfrak{G} \times \mathfrak{G} \rightarrow \mathfrak{G}$, tal que $\mathfrak{G}=\bigoplus_{m=0}^{\infty} G^{m}$, transformando $\mathfrak{G}$ em uma álgebra associativa graduada com 1 .

Como $\pi$ aplica $T_{m}$ em $U_{m}$, podemos compor as aplicações lineares $\phi_{m}: T_{m} \rightarrow U_{m} \rightarrow$ $G^{m}=U_{m} / U_{m-1}$. Ela é sobrejetiva, porque $\pi\left(T_{m}-T_{m-1}\right)=U_{m}-U_{m-1}$. As aplicações $\phi_{m}$ se combinam e produzem uma aplicação linear $\phi: \mathfrak{T} \rightarrow \mathfrak{G}$, que é sobrejetiva e aplica $1 \mathrm{em} 1$.

Lema A.4.1. $\phi: \mathfrak{T} \rightarrow \mathfrak{G}$ é um homomorfismo de álgebras. Além disso, $\phi(I)=\{0\}$. Então $\phi$ induz um homomorfismo $\omega$ de $\mathfrak{S}=\mathfrak{T} /$ I para $\mathfrak{G}$.

Demonstração. [Hum13], Capítulo V, Seção 17.3, pág. 91.

O seguinte Teorema é um resultado básico sobre $U(\mathfrak{g})$; ele (ou seu Corolário A.4.3 ) é chamado de Teorema de Poincaré-Birkhoff-Witt (ou Teorema PBW).

Teorema A.4.1. O homomorfismo $\omega: \mathfrak{S} \rightarrow \mathfrak{G}$ é um isomorfismo de álgebras.

Demonstração. [Hum13], Capítulo V, Seção 17.4, pág. 94.

Corolário A.4.1. Seja $W$ um subespaço de $T^{m}$. Suponha a aplicação canônica $T^{m} \rightarrow S^{m}$ que envia $W$ isomorficamente em $S^{m}$. Então $\pi(W)$ é um complemento para $U_{m-1}$ em $U_{m}$.

Demonstração. [Hum13], Capítulo V, Seção 17.3, pág. 92.

Corolário A.4.2. A aplicação canônica $i: \mathfrak{g} \rightarrow U(\mathfrak{g})$ é injetiva (então $\mathfrak{g}$ pode ser identificada $\operatorname{com} i(\mathfrak{g}))$.

Demonstração. [Hum13], Capítulo V, Seção 17.3, pág. 92.

Corolário A.4.3. Seja $\left\{x_{1}, x_{2}, x_{3}, \ldots\right\}$ qualquer base ordenada de g. Então os elementos $x_{i(1)} \cdots x_{i(m)}=\pi\left(x_{i(1)} \otimes \cdots \otimes x_{i(m)}\right), m \in \mathbb{Z}^{+}, i(1) \leq i(2) \leq \ldots \leq i(m)$, juntamente com 1 , formam uma base para $U(\mathfrak{g})$.

Demonstração. [Hum13], Capítulo V, Seção 17.3, pág. 92.

Corolário A.4.4. Seja $\mathfrak{h}$ uma subálgebra de $\mathfrak{g}$, e estenda uma base ordenada $\left\{h_{1}, h_{2}, \ldots\right\}$ de $\mathfrak{h}$ para uma base ordenada $\left\{h_{1}, \ldots, x_{1}, \ldots\right\}$ de $\mathfrak{g}$. Então o homomorfismo $U(\mathfrak{h}) \rightarrow U(\mathfrak{g})$ induzido pela injeção $\mathfrak{h} \rightarrow \mathfrak{g} \rightarrow U(\mathfrak{g})$ é também injetivo, e $U(\mathfrak{g})$ é um $U(\mathfrak{h})$-módulo livre com base consistindo de todos $x_{i(1)} \cdots x_{i(m)}, i(1) \leq i(2) \leq \ldots \leq i(m)$, juntamente com 1 .

Demonstração. [Hum13], Capítulo $V$, Seção 17.3, pág. 92.

Observação A.4.1. A finitude da dimensão da álgebra de Lie $\mathfrak{g}$ não implica na finitude da dimensão da sua álgebra envolvente universal. Por exemplo, a álgebra de Lie $\mathfrak{g l}(n)$ é gerada pelas matrizes elementares $e_{i j}$, com $1 \leq i, j \leq n$, e portanto possui dimensão $n^{2}$. Por outro lado, $U(\mathfrak{g l}(n))$ é, segundo o Teorema $P B W$, gerada por monômios do tipo $e_{11}^{\alpha_{11}} \cdot e_{12}^{\alpha_{12}} \ldots e_{n n}^{\alpha_{n n}}$, e como há infinitos monômios desse tipo, concluímos que a dimensão da álgebra envolvente não é finita. 


\section{A.5 Referências}

A Seção A.1 foi baseada em [Rot03][Capítulos 3, 6 e 7] e [Lam01]. As Seções A.2, A.3 e A.4 foram baseadas em [Hum13]. 
114 APÊNDICE A 


\section{Apêndice B}

\section{Teoria de Categorias}

A teoria de categorias foi fundada por Saunders Mac Lane e Samuel Eilenberg por volta de 1940. É uma teoria razoavelmente abstrata que aparentemente não tem nenhum conteúdo, razão pela qual foi batizada de "absurdo abstrato". No entanto, é uma linguagem muito flexível e poderosa, que tem se tornado totalmente indispensável em muitas áreas da matemática, tal como geometria algébrica, topologia, teoria de representações, e muitas outras.

Observamos que muitas propriedades matemáticas podem ser unificadas e simplificadas por uma apresentação com diagramas de flechas. Além disso, muitas propriedades matemáticas podem ser representadas por propriedades universais de diagramas. Intuitivamente, uma categoria pode ser pensada como um aglomerado de "pontos e flechas", em que as "flechas" satisfazem certas propriedades. Os "pontos" são os objetos da categoria e as "flechas" são os morfismos.

Neste Apêndice estão as definições básicas da teoria geral de categorias e de alguns resultados já clássicos. Os resultados apresentados aqui não serão provados. Para um conhecimento mais profundo e maiores detalhes, o leitor deve utilizar o livro clássico "Categories for the Working Mathematician" [Lan98]. Também utilizaremos ao longo do Apêndice os seguintes livros: "Introduction to Representation Theory" [Eti11], "A first course of homological algebra" [Nor73], "Advanced modern algebra" [Rot03] e "An Introduction to Homological Algebra" [Rot09].

\section{B.1 Categorias e Funtores}

Definição B.1.1. Uma categoria $\mathcal{C}$ é o seguinte conceito:

1. Uma classe de objetos $\mathrm{Ob}(\mathcal{C})$;

2. Para todos objetos $X, Y \in O b(\mathcal{C})$, existe uma classe $\operatorname{Hom}_{\mathcal{C}}(X, Y)=\operatorname{Hom}(X, Y)$ de morfismos (ou flechas) de $X$ para $Y$ (para $f \in \operatorname{Hom}(X, Y)$, podemos escrever $f: X \rightarrow$ $Y)$. Também podemos denotar essa classe por $\mathcal{C}(X, Y)$;

3. Para todos objetos $X, Y, Z \in O b(\mathcal{C})$, existe uma aplicação composição

$$
\circ: \operatorname{Hom}(Y, Z) \times \operatorname{Hom}(X, Y) \rightarrow \operatorname{Hom}(X, Z)
$$

tal que $(f, g) \mapsto f \circ g$, que satisfaz os seguintes axiomas:

(a) A composição é associativa, isto é, $(f \circ g) \circ h=f \circ(g \circ h)$, para quaisquer $f, g, h$ para quais a composição faça sentido; 
(b) Para cada $X \in O b(\mathcal{C})$, existe um morfismo $1_{X}$ ou $i d_{X} \in \operatorname{Hom}(X, X)$, chamado de morfismo unitário ou morfismo identidade, tal que $1_{X} \circ f=f$ e $g \circ 1_{X}=g$, para quaisquer $f, g$ para quais a composição faça sentido;

(c) Se os pares $(X, Y)$ e $\left(X^{\prime}, Y^{\prime}\right)$ são distintos, então a intersecção dos conjuntos $\operatorname{Hom}(X, Y)$ e $\operatorname{Hom}\left(X^{\prime}, Y^{\prime}\right)$ é vazia.

Observação B.1.1. Nós escreveremos $X \in \mathcal{C}$, ao invés de $X \in O b(\mathcal{C})$.

Exemplo B.1.1. Seguem alguns exemplos de categorias:

1. A categoria Sets. Os objetos da categoria Sets são conjuntos. Se $X, Y$ são dois conjuntos, podemos definir $\operatorname{Hom}_{\text {Sets }}(X, Y)$, a classe de todas as aplicações arbitrárias definidas de $X$ para $Y$. A composição dos morfismos é a composição usual de aplicações.

2. A categoria Groups. Os objetos da categoria Groups são grupos. O morfismo definido entre dois objetos é um homomorfismo de grupos. A composição dos morfismos é a composição entre dois homomorfismos de grupos.

3. A categoria AbGroups, cujos objetos são os grupos abelianos e os morfismos são homomorfismos de grupos.

4. A categoria Rings. Os objetos da categoria Rings são anéis. O morfismo definido entre dois objetos é um homomorfismo de anéis. A composição dos morfismos é a composição entre dois homomorfismos de anéis.

5. A categoria Vect $_{k}$, que tem como objetos os espaços vetoriais sobre um corpo $k$. O morfismo entre dois objetos é uma aplicação linear e a composição entre dois morfismos é a composição entre duas aplicações lineares.

6. A categoria $R$-mod dos módulos à esquerda sobre um anel $R$. Os morfismos são homomorfismos de módulos sobre $R$. A composição entre morfismos é a composição entre homomorfismos de $R$-módulos. De maneira análoga mod- $R$ é a categoria dos módulos à direita sobre um anel $R$.

7. A categoria Top, em que os objetos são espaços topológicos. Se $X, Y$ são dois espaços topológicos, podemos definir $\operatorname{Hom}_{\text {Top }}(X, Y)$, a classe de todas as aplicações contínuas definidas de $X$ para $Y$. A composição dos morfismos é a composição usual de aplicações contínuas.

8. A categoria hTop de homotopia de todos os espaços topológicos. Os morfismos são as classes de homotopia das aplicações contínuas e os objetos são os espaços topológicos.

Infelizmente, não podemos simplificar esta definição substituindo a palavra "classe", por uma mais familiar, "conjunto". Na verdade, isto excluiria o importante Exemplo B.1.1(1), pois como é bem conhecido, não existe conjunto de todos os conjuntos, e trabalhar com um tal conjunto leva à contradições. A definição precisa de uma classe e a distinção exata entre uma classe e um conjunto é assunto da teoria dos conjuntos, e não será discutido aqui. Felizmente, para fins mais práticos, esta distinção não é essencial.

Definição B.1.2. Se $O b(\mathcal{C})$ de uma categoria $\mathcal{C}$ é um conjunto e $\operatorname{Hom}_{\mathcal{C}}(X, Y)$ são conjuntos para todos $X, Y \in \mathcal{C}$, dizemos que a categoria é pequena. Se somente a segunda condição é satisfeita dizemos que a categoria é localmente pequena. 
Nós podemos mencionar que muitos exemplos, incluindo os Exemplos B.1.1(1) à B.1.1(8), são categorias localmente pequenas.

Definição B.1.3. Seja $\mathcal{C}$ uma categoria. Uma subcategoria $\mathcal{C}^{\prime}$ de $\mathcal{C}$, é uma categoria que satisfaz as seguintes condições:

1. $O b\left(\mathcal{C}^{\prime}\right) \subseteq O b(\mathcal{C})$;

2. $\operatorname{Hom}_{\mathcal{C}^{\prime}}(X, Y) \subseteq \operatorname{Hom}_{\mathcal{C}}(X, Y)$, para todos $X, Y \in \mathcal{C}^{\prime}$;

3. A composição em $\mathcal{C}^{\prime}$ é induzida pela composição em $\mathcal{C}$;

4. Os morfismos identidade em $\mathcal{C}^{\prime}$ são morfismos identidade em $\mathcal{C}$.

Exemplo B.1.2. 1. Top é uma subcategoria de Sets.

2. Groups é uma subcategoria de Sets.

3. AbGroups é uma subcategoria de Groups.

Definição B.1.4 (Subcategoria Plena). Seja $\mathcal{D}$ uma subcategoria de $\mathcal{C}$. Dizemos que $\mathcal{D}$ é uma subcategoria plena de $\mathcal{C}$ se, para todos $X, Y \in \mathcal{D}$

$$
\operatorname{Hom}_{\mathcal{D}}(X, Y)=\operatorname{Hom}_{\mathcal{C}}(X, Y)
$$

No Exemplo B.1.2(3) temos uma subcategoria plena, pois um morfismo entre dois grupos abelianos não depende se você considera eles como grupos abelianos ou simplesmente grupos. Já os Exemplos B.1.2(1) e B.1.2(2), não são subcategorias plenas, pois os morfismos da categoria Sets são aplicações arbitrárias que não serão necessariamente contínuas ou homomorfismos de grupos.

Definição B.1.5 (Categoria Produto). Se $\left(\mathcal{C}_{i}\right)_{i \in I}$ é uma familia de categorias, definimos a categoria produto $\prod_{i \in I} \mathcal{C}_{i}$ como segue:

1. Os objetos de $\prod_{i \in I} \mathcal{C}_{i}$ são todos da forma $\left(X_{i}\right)_{i \in I}$, tal que para cada $i \in I, X_{i}$ é objeto de $\mathcal{C}_{i}$

2. $\operatorname{Hom}_{i \in I} \mathcal{C}_{i}\left(\left(X_{i}\right)_{i \in I},\left(Y_{i}\right)_{i \in I}\right)=\prod_{i \in I} \operatorname{Hom}_{\mathcal{C}_{i}}\left(X_{i}, Y_{i}\right)$;

3. Se $\left(u_{i}\right)_{i \in I} \in \operatorname{Hom}_{i \in I} \mathcal{C}_{i}\left(\left(X_{i}\right)_{i \in I},\left(Y_{i}\right)_{i \in I}\right) e\left(v_{i}\right)_{i \in I} \in \operatorname{Hom}_{\prod_{i \in I} \mathcal{C}_{i}}\left(\left(Y_{i}\right)_{i \in I},\left(Z_{i}\right)_{i \in I}\right)$ então $\left(v_{i}\right)_{i \in I} \circ\left(u_{i}\right)_{i \in I}=\left(v_{i} \circ u_{i}\right)_{i \in I} \in \operatorname{Hom}_{i \in I} \mathcal{C}_{i}\left(\left(X_{i}\right)_{i \in I},\left(Z_{i}\right)_{i \in I}\right)$.

Dada uma categoria, podemos obter novas categorias de várias maneiras. Uma maneira é inverter todas as "flechas". Uma definição mais precisa é:

Definição B.1.6 (Categoria Oposta). Dada uma categoria $\mathcal{C}$, definimos seu oposto, denotando por $\mathcal{C}^{o p}$, como a categoria com os mesmos objetos de $\mathcal{C}$, mas com $\operatorname{Hom}_{\mathcal{C}^{o p}}(X, Y)=$ $\operatorname{Hom}_{\mathcal{C}}(Y, X)$. A composição nesta categoria é definida como segue: seja $f^{*} \in \operatorname{Hom}_{C^{o p}}(X, Y)$ $\left(\operatorname{com} f \in \operatorname{Hom}_{\mathcal{C}}(Y, X)\right)$, então a composição com $g^{*} \in \operatorname{Hom}_{\mathcal{C}^{o p}}(Y, Z)\left(\operatorname{com} g \in \operatorname{Hom}_{\mathcal{C}}(Z, Y)\right)$ é dada por

$$
g^{*} \circ f^{*}=(f \circ g)^{*}
$$


Observação B.1.2. Fazendo duas vezes a inversão, temos a categoria original, de modo que o oposto de uma categoria oposta é a própria categoria original, isto é, $\left(\mathcal{C}^{o p}\right)^{o p}=\mathcal{C}$.

Definição B.1.7 (Pullback / Produto Fibrado). Sejam $\mathcal{C}$ um categoria e $f_{1}: X_{1} \rightarrow X$ e $f_{2}: X_{2} \rightarrow X$ dois morfismos, com $X, X_{1}, X_{2} \in \mathcal{C}$. $O$ pullback ou produto fibrado do par $\left(f_{1}, f_{2}\right)$ é uma tripla $\left(W, h_{1}, h_{2}\right)$, em que $W \in \mathcal{C}$ e $h_{1}: W \rightarrow X_{1}, h_{2}: W \rightarrow X_{2}$ são morfismos em $\mathcal{C}$ tais que:

1. $f_{1} \circ h_{1}=f_{2} \circ h_{2}$;

2. Para todo objeto $Z$ de $\mathcal{C}$ e todo par de morfismos $g_{1}: Z \rightarrow X_{1}$ e $g_{2}: Z \rightarrow X_{2}$ tal que $f_{1} \circ g_{1}=f_{2} \circ g_{2}$, existe um único morfismo $g: Z \rightarrow W$ tal que $h_{1} \circ g=g_{1}$ e $h_{2} \circ g=g_{2}$.

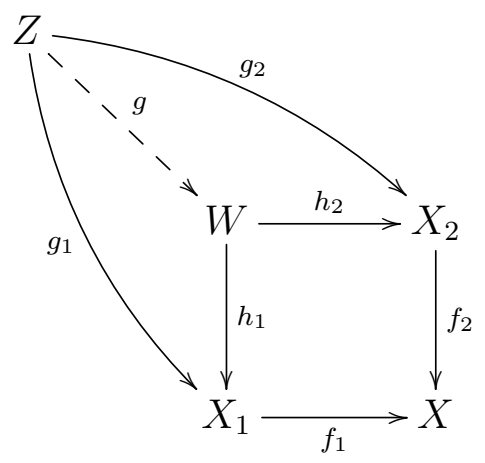

Segue da propriedade 2. da Definição B.1.7 que, quando existe um pullback $\left(W, h_{1}, h_{2}\right)$ do par $\left(f_{1}, f_{2}\right)$, este é único a menos de isomorfismos. Por simplicidade, quando não houver ambiguidade, diremos apenas que o objeto $W$ é o produto fibrado de $f_{1}$ e $f_{2}$.

Proposição B.1.1. O pullback de dois morfismos $f: M \rightarrow N$ e $g: M^{\prime} \rightarrow N$ em $R$-mod existe.

Demonstração. [Rot03], Capítulo 7, Proposição 7.36, pág. 456.

Definição B.1.8 (Pushout). Sejam $\mathcal{C}$ um categoria e $f: Z \rightarrow X$ e $g: Z \rightarrow Y$ dois morfismos, com $X, Y, Z \in \mathcal{C}$. O pushout do par $(f, g)$ é uma tripla $\left(P, i_{1}, i_{2}\right)$, em que $P \in$ $\mathcal{C}$ e $i_{1}: X \rightarrow P, i_{2}: Y \rightarrow P$ são morfismos em $\mathcal{C}$ tais que:

1. $i_{1} \circ f=i_{2} \circ g$;

2. Para todo objeto $Q$ de $\mathcal{C}$ e todo par de morfismos $j_{1}: X \rightarrow Q$ e $j_{2}: Y \rightarrow Q$ tal que $j_{1} \circ f=j_{2} \circ g$, existe um único morfismo $u: P \rightarrow Q$ tal que $u \circ i_{1}=j_{1}$ e $u \circ i_{2}=j_{2}$.

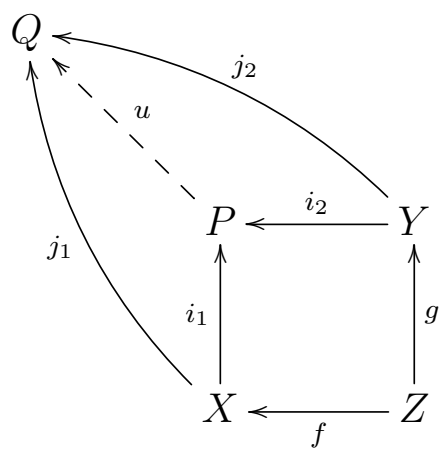

Segue da propriedade 2. da Definição B.1.8 que, quando existe um pushout $\left(P, i_{1}, i_{2}\right)$ do par $(f, g)$, este é único a menos de isomorfismos. O pushout é o dual do pullback, e por conta disso também é conhecido como coproduto desfibrado. 
Proposição B.1.2. O pushout de dois morfismos $f: M \rightarrow N$ e $g: M \rightarrow N^{\prime}$ em $R$-mod existe.

Demonstração. [Rot03], Capítulo 7, Proposição 7.38, pág. 457.

Agora uma definição sobre os tipos de morfismos nas categorias:

Definição B.1.9 (Monomorfismo/Epimorfismo/Isomorfismo). Seja $\mathcal{C}$ uma categoria e $X, Y, Z$ $\in \mathcal{C}$. Um morfismo $f \in \operatorname{Hom}_{\mathcal{C}}(Y, Z)$ é um monomorfismo se para todos $g, h \in \operatorname{Hom}_{\mathcal{C}}(X, Y)$ com $f \circ g=f \circ h$, temos que $g=h$. Um morfismo $f \in \operatorname{Hom}_{\mathcal{C}}(X, Y)$ é um epimorfismo se para todos $g, h \in \operatorname{Hom}_{\mathcal{C}}(Y, Z)$ com $g \circ f=h \circ f$, temos que $g=h$. Um morfismo $f \in \operatorname{Hom}_{\mathcal{C}}(X, Y)$ é um isomorfismo se existe um $g \in \operatorname{Hom}_{\mathcal{C}}(Y, X)$ tal que $f \circ g=i d_{Y}$ e $g \circ f=i d_{X}$.

Definição B.1.10 (Objeto Inicial/Terminal). Seja $\mathcal{C}$ uma categoria. Um objeto I em $\mathcal{C}$ é chamado inicial se $\operatorname{Hom}_{\mathcal{C}}(I, X)$ tem um único elemento para todo $X$ em $\mathcal{C}$. Analogamente, um objeto terminal $T$ em $\mathcal{C}$ é tal que $\operatorname{Hom}_{\mathcal{C}}(X, T)$ consiste de um único elemento para todo $X$ em $\mathcal{C}$. Um objeto que é tanto inicial quanto terminal é chamado de zero ou objeto nulo.

Nem toda categoria tem objeto inicial ou terminal (ou ambos).

Exemplo B.1.3. 1. A categoria Groups tem como objeto inicial e terminal o grupo unitário $\{e\}$, em que $e$ é o elemento neutro.

2. A categoria $R$-mod tem como objeto inicial e terminal o $R$-módulo nulo $\{0\}$.

3. A categoria Field dos corpos, não tem nem objeto inicial nem objeto terminal.

Quando dois objetos algébricos ou geométricos são isomorfos, normalmente não é uma boa ideia dizer que eles são iguais ou literalmente a mesma coisa. A razão é que tais objetos são normalmente iguais de muitas maneiras diferentes, isto é, há muitas maneiras de tomar um isomorfismo entre eles.

Dois objetos $X$ e $Y$ de $\mathcal{C}$ são ditos isomorfos se existe um isomorfismo $f: X \rightarrow Y$. Quaisquer dois objetos iniciais (respectivamente, finais ou nulos) de $\mathcal{C}$ são isomorfos. Dois objetos isomorfos tem essencialmente as mesmas propriedades. Obviamente, a relação "isomorfismo" é de fato uma relação de equivalência em $O b(\mathcal{C})$. A classe dos objetos que são isomorfos à $X$ é denotada por $[X]$ e será chamada de tipo de $X$. Uma nova categoria pode ser construída pelos tipos de objetos de uma categoria $\mathcal{C}$, e esta categoria será chamada de categoria tipo de $\mathcal{C}$ ou esqueleto de $\mathcal{C}$. Claramente, a categoria tipo de $\mathcal{C}$ tem as mesmas propriedades de $\mathcal{C}$.

Definição B.1.11 (Funtor Covariante/Contravariante). Sejam $\mathcal{C}$ e $\mathcal{D}$ categorias. Um funtor covariante $F: \mathcal{C} \rightarrow \mathcal{D}$ é um par de aplicações:

1. Uma aplicação $F: O b(\mathcal{C}) \longrightarrow O b(\mathcal{D})$, tal que $X \mapsto F(X)$, para cada $X \in \mathcal{C}$.

2. Uma aplicação $F_{X, Y}: \operatorname{Hom}_{\mathcal{C}}(X, Y) \longrightarrow \operatorname{Hom}_{\mathcal{D}}(F(X), F(Y))$, para cada $X, Y \in \mathcal{C}$.

tal que $F\left(i d_{X}\right)=i d_{F(X)}$, para cada $X \in \mathcal{C}$ e $F(f \circ g)=F(f) \circ F(g)$ (escrevemos simplificadamente $F(f)$ ao invés de $\left.F_{X, Y}(f)\right)$. Se $F_{X, Y}: \operatorname{Hom}_{\mathcal{C}}(X, Y) \rightarrow \operatorname{Hom}_{\mathcal{D}}(F(Y), F(X))$ e $F(f \circ g)=F(g) \circ F(f), F$ é chamado de funtor contravariante. 
Claramente, um funtor covariante de $\mathcal{C}$ para $\mathcal{D}^{o p}$ e um funtor covariante de $\mathcal{C}^{o p}$ para $\mathcal{D}$ são naturalmente associados cada um com um funtor contravariante de $\mathcal{C}$ para $\mathcal{D}$ e reciprocamente. Então, o estudo geral dos funtores contravariantes pode ser reduzido ao estudo dos funtores covariantes.

Notamos que funtores podem ser compostos de maneira óbvia. Temos também que qualquer categoria tem o funtor identidade $1_{\mathcal{C}}: \mathcal{C} \rightarrow \mathcal{C}$, que leva um objeto $X$ em $X$ e um morfismo $f \in \operatorname{Hom}_{\mathcal{C}}(X, Y)$ em $f \in \operatorname{Hom}_{\mathcal{C}}(X, Y)$.

Exemplo B.1.4. Seguem alguns exemplos de funtores:

1. Funtor Esquecimento: é um funtor que "perde" ou "esquece" na sua imagem, parte da estrutura de uma categoria. Seja $F_{1}:$ Top $\rightarrow$ Sets, tal que para cada espaço topológico $X, F_{1}(X)$ é o conjunto subjacente e para cada aplicação contínua $f, F_{1}(f)$ é a aplicação correspondente. $F_{1}$ é um funtor esquecimento. Também temos como exemplos $F_{2}:$ Groups $\rightarrow$ Sets, $F_{3}:$ Rings $\rightarrow$ AbGroups.

2. $V \mapsto V^{*}$, em que $V^{*}$ é o espaço dos funcionais lineares de $V$ em $k$ (corpo), é um funtor de

$\operatorname{Vect}_{k} \rightarrow \operatorname{Vect}_{k}^{o p}$

3. Os funtores Hom: se $\mathcal{C}$ é uma categoria localmente pequena, então temos o funtor $\operatorname{Hom}(X, \cdot): \mathcal{C} \rightarrow$ Sets, dado por $Y \mapsto \operatorname{Hom}(X, Y)$. Temos também o funtor $\operatorname{Hom}(\cdot, X)$ : $\mathcal{C} \rightarrow$ Sets, dado por $Y \mapsto \operatorname{Hom}(Y, X)$.

4. Seja $Q$ um quiver. Considere a categoria $\mathcal{C}(Q)$, cujos objetos são os vértices e os morfismos são os caminhos orientados entre eles. Então funtores de $\mathcal{C}(Q)$ em $\operatorname{Vect}_{k}$, são representações de $Q$ sobre $k$.

5. Os funtores de soma direta e produto tensorial, são funtores de $\operatorname{Vect}_{k} \times \operatorname{Vect}_{k} \rightarrow$ Vect $_{k}$. As operações $V \mapsto V^{\otimes n}, V \mapsto S^{n} V$ e $V \mapsto \wedge^{n} V$, também são funtores em $\operatorname{Vect}_{k}$.

Definição B.1.12 (Transformação Natural). Sejam $\mathcal{C}, \mathcal{D}$ categorias e $F, G$ dois funtores de $\mathcal{C}$ para $\mathcal{D}$. Uma transformação natural de $F$ em $G$ (ou morfismo funtorial) é uma função $\eta: F \rightarrow G$, tal que para todo $X \in \mathcal{C}$ existe um morfismo $\eta_{X}: F(X) \rightarrow G(X)$, ou seja,

$$
\mathcal{C} \ni X \longmapsto \eta_{X} \in \operatorname{Hom}_{\mathcal{D}}(F(X), G(X))
$$

tal que para todos $X, X^{\prime} \in \mathcal{C}$ e para todo $f \in \operatorname{Hom}_{\mathcal{C}}\left(X, X^{\prime}\right)$ o diagrama

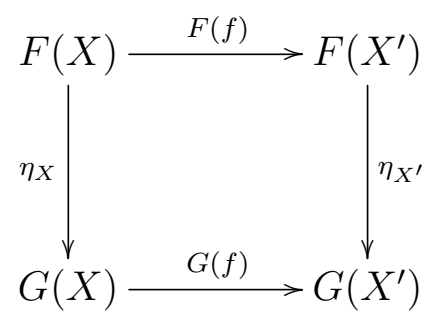

comuta.

Uma das características importantes de funtores entre categorias que os distingue das aplicações ou funções habituais é que a classe de todos os funtores entre duas categorias dadas formam uma categoria, pois podemos definir de modo não trivial um morfismo entre dois funtores dessa classe, isto é, entre dois funtores dados de uma categoria $\mathcal{C}$ em uma categoria $\mathcal{D}$ existe uma transformação natural. 
Observação B.1.3. Se $T: \mathcal{C} \rightarrow \mathcal{D}$ é um funtor covariante, $X, Y \in \mathcal{C}$ e $f \in \operatorname{Hom}_{\mathcal{C}}(X, Y)$ é um isomorfismo, então $T(f) \in \operatorname{Hom}_{\mathcal{D}}(T(X), T(Y))$ é um isomorfismo. O mesmo acontece se $T$ for contravariante. Portanto os funtores preservam os isomorfismos.

Definição B.1.13 (Funtor totalmente fiel). Um funtor $F: \mathcal{C} \rightarrow \mathcal{D}$ é dito pleno se para todos $X, Y \in \mathcal{C}$ e para todo $g \in \operatorname{Hom}_{\mathcal{D}}(F(X), F(Y))$, existe $f \in \operatorname{Hom}_{\mathcal{C}}(X, Y)$ tal que $g=F(f)$. Um funtor $F: \mathcal{C} \rightarrow \mathcal{D}$ é dito fiel se para todos $X, Y \in \mathcal{C}$ e para todos $f, g \in \operatorname{Hom}_{\mathcal{C}}(X, Y)$ a igualdade $F(f)=F(g)$ implica $f=g$. Um funtor $F: \mathcal{C} \rightarrow \mathcal{D}$ é dito totalmente fiel se é pleno e fiel, ou seja, se para todos $X, Y \in \mathcal{C}$, a aplicação

$$
F_{X, Y}: \operatorname{Hom}_{\mathcal{C}}(X, Y) \rightarrow \operatorname{Hom}_{\mathcal{D}}(F(X), F(Y))
$$

é uma bijeção.

Em outras palavras: para $X, Y \in \mathcal{C}$ temos uma aplicação

$$
F_{X, Y}: \operatorname{Hom}_{\mathcal{C}}(X, Y) \rightarrow \operatorname{Hom}_{\mathcal{D}}(F(X), F(Y))
$$

tal que $f \mapsto F(f)$. Então $F$ é pleno se para todos $X, Y \in \mathcal{C}$, a aplicação $F_{X, Y}$ é sobrejetora e é fiel se para todos $X, Y \in \mathcal{C}$, a aplicação $F_{X, Y}$ é injetora. Observamos que a composição de funtores plenos é um funtor pleno e a composição de funtores fiéis é um funtor fiel.

Existe a noção de isomorfismo de categorias: dizemos que um funtor $F: \mathcal{C} \rightarrow \mathcal{D}$ é um isomorfismo de categorias se existe outro funtor $G: \mathcal{D} \rightarrow \mathcal{C}$ tal que $G \circ F$ é o funtor identidade em $\mathcal{C}$ e $F \circ G$ é o funtor identidade em $\mathcal{D}$.

Definição B.1.14 (Equivalência de Categorias). Um funtor $F: \mathcal{C} \rightarrow \mathcal{D}$ é uma equivalência de categorias (e as categorias $\mathcal{C}$ e $\mathcal{D}$ são equivalentes) se existe $G: \mathcal{D} \rightarrow \mathcal{C}$ tal que $F \circ G e ́$ isomorfo à $1_{\mathcal{D}}$ e $G \circ F$ é isomorfo à $1_{\mathcal{C}}$.

Definição B.1.15 (Funtor Representável). Seja $\mathcal{C}$ uma categoria localmente pequena e $F$ : $\mathcal{C} \rightarrow$ Sets um funtor. Dizemos que $F$ é um funtor representável se existe um objeto $X \in \mathcal{C}$ tal que $F$ é isomorfo ao funtor $\operatorname{Hom}(X, \cdot)$. Mais precisamente, se nos é dado um objeto $X$ $\in \mathcal{C}$, junto com um isomorfismo $\xi: F \cong \operatorname{Hom}(X, \cdot)$, dizemos que o funtor $F$ é representado por $X$ (usando $\xi)$.

Lema B.1.1 (Lema de Yoneda). Se um funtor $F$ é representado por um objeto $X$, então $X$ é único a menos de isomorfismo, isto é, se $X, Y$ são dois objetos em $\mathcal{C}$, então para qualquer isomorfismo de funtores $\phi: \operatorname{Hom}(X, \cdot) \rightarrow \operatorname{Hom}(Y, \cdot)$ existe um único isomorfismo $a_{\phi}: X \rightarrow Y$ induzido por $\phi$.

Demonstração. [Eti11], Capítulo 7, Lema 7.5.1, pág. 184.

Outra noção fundamental em teoria de categorias é a noção de funtores adjuntos:

Definição B.1.16 (Funtores Adjuntos). Os funtores $F: \mathcal{C} \rightarrow \mathcal{D}$ e $G: \mathcal{D} \rightarrow \mathcal{C}$ são um par de funtores adjuntos se para todos $X \in \mathcal{C}, Y \in \mathcal{D}$, temos um isomorfismo

$$
\xi_{X Y}: \operatorname{Hom}_{\mathcal{D}}(F(X), Y) \rightarrow \operatorname{Hom}_{\mathcal{C}}(X, G(Y))
$$

que é funtorial em $X$ e $Y$, ou seja, se temos um isomorfismo de funtores $\operatorname{Hom}(F(\cdot), \cdot) \rightarrow$ $\operatorname{Hom}(\cdot, G(\cdot))$, em que $\operatorname{Hom}(F(\cdot), \cdot): \mathcal{C} \times \mathcal{D} \rightarrow \operatorname{Sets}, \operatorname{Hom}(\cdot, G(\cdot)): \mathcal{C} \times \mathcal{D} \rightarrow$ Sets. Nesta situação dizemos que $F$ é adjunto à esquerda para $G$ e $G$ é adjunto à direita para $F$. 
Nem todo funtor tem adjunto à esquerda ou à direita, mas se ele existe, ele é único e pode ser construído canonicamente (isto é, se de alguma forma encontro esses dois funtores, então existe um isomorfismo canônico entre eles). Isto segue do Lema de Yoneda B.1.1, pois se $F, G$ são um par de funtores adjuntos, então $F(X)$ representa o funtor $Y \mapsto \operatorname{Hom}(X, G(Y))$ e $G(Y)$ representa o funtor $X \mapsto \operatorname{Hom}(F(X), Y)$.

\section{B.2 Categorias Aditivas e Abelianas}

Os tipos de categorias que mais aparecem em teoria de representações são as categorias abelianas.

Definição B.2.1 (Coproduto). Sejam $\mathcal{C}$ uma categoria e $X, Y$ objetos da categoria $\mathcal{C}$. $O$ coproduto de $X$ e $Y$, denotado por $X+Y$ ou $X \sqcup Y$, é um objeto $Z \in \mathcal{C}$ munido com morfirmos injetivos $\iota_{1}: X \rightarrow Z, \iota_{2}: Y \rightarrow Z$, tal que, para todo $W \in \mathcal{C}$ e para todo par de morfismos $\phi_{1}: X \rightarrow W, \phi_{2}: Y \rightarrow W$, existe um único morfismo $\psi: Z \rightarrow W$ que fazo seguinte diagrama comutar (isto é, $\psi \circ \iota_{1}=\phi_{1}$ e $\psi \circ \iota_{2}=\phi_{2}$ ):

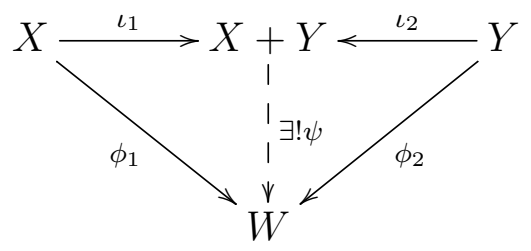

Nem sempre o coproduto existe. Podemos construir exemplos de categorias em que um par de objetos não tem coproduto. (Veja [Rot03], Exercício 7.21, pág. 458). Nas categorias Sets, Groups e $R$-mod o coproduto sempre existe. O coproduto de dois objetos em Sets é chamado de união disjunta de conjuntos, em Groups é chamado de produto livre e em $R$-mod é chamado de soma direta.

Proposição B.2.1. Sejam $M$ e $N$, R-módulos à esquerda. Então o coproduto entre $M$ e $N$ existe em $R$-mod e ele é a soma direta.

Demonstração. [Rot03], Capítulo 7, Proposição 7.26, pág. 448.

Proposição B.2.2. Sejam $\mathcal{C}$ uma categoria e $X, Y$ objetos da categoria $\mathcal{C}$. Então quaisquer dois coprodutos de $X$ e $Y$, caso existam, são equivalentes.

Demonstração. [Rot03], Capítulo 7, Proposição 7.27, pág. 448.

Definição B.2.2 (Produto). Sejam $\mathcal{C}$ uma categoria e $X, Y$ objetos da categoria $\mathcal{C}$. $O$ produto de $X$ e $Y$, denotado por $X \times Y$ ou $X \sqcap Y$, é um objeto $P \in \mathcal{C}$ munido com projeções $\pi_{1}: P \rightarrow X, \pi_{2}: P \rightarrow Y$, tal que, para cada objeto $Q \in \mathcal{C}$ e para todo par de morfismos $\phi_{1}: Q \rightarrow X, \phi_{2}: Q \rightarrow Y$, existe um único morfismo $\psi: Q \rightarrow P$ que faz o seguinte diagrama comutar (isto é, $\pi_{1} \circ \psi=\phi_{1}$ e $\pi_{2} \circ \psi=\phi_{2}$ ):

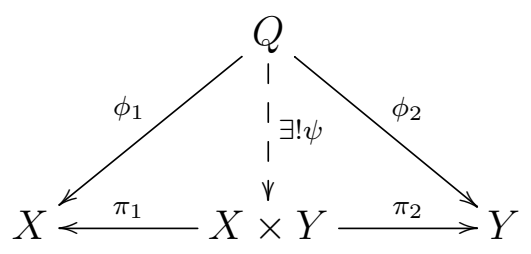

O produto de dois objetos existe, entre outras, nas categorias $R$-mod, Groups e Top. Nestes casos, e em muitos outros, é chamado de produto direto. 
Proposição B.2.3. Sejam $\mathcal{C}$ uma categoria e $X, Y$ objetos da categoria $\mathcal{C}$. Então quaisquer dois produtos de $X$ e $Y$, caso existam, são equivalentes.

Demonstração. [Rot03], Capítulo 7, Proposição 7.28, pág. 450.

Definição B.2.3 (Biproduto). Sejam $\mathcal{C}$ uma categoria e $X, Y$ objetos da categoria $\mathcal{C}$. Suponhamos que $\mathcal{C}$ tem um objeto zero. Um biproduto para $X$ e $Y$ é um objeto $B$, geralmente denotado por $X \oplus Y$, de $\mathcal{C}$ que é o produto e o coproduto de $X$ e $Y$, e para qual a coleção de morfismos $\pi_{i}$ e $\iota_{i}, i=1,2$, satisfazem: $\pi_{1} \circ \iota_{1}=i d_{X}, \pi_{2} \circ \iota_{2}=i d_{Y}, \pi_{i} \circ \iota_{j}=0$ se $i \neq j$, e $\iota_{1} \circ \pi_{1}+\iota_{2} \circ \pi_{2}=i d_{B}$. Segue o diagrama do biproduto de $X$ e $Y$ :

$$
X \underset{\pi_{1}}{\stackrel{\iota_{1}}{\gtrless}} X \oplus Y \underset{\iota_{2}}{\stackrel{\pi_{2}}{\gtrless}} Y
$$

Seja $\mathcal{C}$ uma categoria localmente pequena, ou seja, para todos $X, Y \in \mathcal{C}, \operatorname{Hom}_{\mathcal{C}}(X, Y)$ é um conjunto. Em muitos exemplos os conjuntos $\operatorname{Hom}_{\mathcal{C}}(X, Y)$ tem alguma estrutura adicional. Por exemplo, na categoria Vect Ve $_{k}$ para $V, W$ espaços vetoriais sobre $k$, cada $\operatorname{Hom}_{\mathbf{V e c t}_{k}}(V, W)$, o conjunto de todas as aplicações lineares de $V$ para $W$, é um espaço vetorial sobre $k$. Um caso mais simples é quando para todo $X, Y \in \mathcal{C}$, os conjuntos $\operatorname{Hom}_{\mathcal{C}}(X, Y)$ são grupos abelianos aditivos:

Definição B.2.4 (Categoria Pré-aditiva/Ab-categoria). Seja $\mathcal{C}$ uma categoria. $\mathcal{C}$ será uma categoria pré-aditiva (ou Ab-categoria) se cada $\operatorname{Hom}_{\mathcal{C}}(X, Y)$, com $X, Y \in \mathcal{C}$, é um grupo abeliano aditivo e a composição o é bilinear, isto é:

1. Para todos $f, g \in \operatorname{Hom}_{\mathcal{C}}(X, Y)$ e $p \in \operatorname{Hom}_{\mathcal{C}}(Y, Z)$ temos que $p \circ(f+g)=p \circ f+p \circ g$ $\in \operatorname{Hom}_{\mathcal{C}}(X, Z)$;

2. Para todos $f, g \in \operatorname{Hom}_{\mathcal{C}}(X, Y)$ e $q \in \operatorname{Hom}_{\mathcal{C}}(Z, X)$ temos que $(f+g) \circ q=f \circ q+g \circ q$ $\in \operatorname{Hom}_{\mathcal{C}}(Z, Y)$.

Temos que AbGroups, $R$-mod, $\bmod -R$ são todas Ab-categorias.

Teorema B.2.1. Dois objetos $X$ e $Y$ em uma $A$ b-categoria $\mathcal{C}$ tem um produto em $\mathcal{C}$ se, e somente se, tem um biproduto em $\mathcal{C}$. Especificamente, dado um biproduto $B$ de $X$ e $Y$, o objeto $B$ com os morfismos $\pi_{1}, \pi_{2}$ é um produto de $X$ e $Y$, enquanto que, $B$ com os morfismos $\iota_{1}, \iota_{2}$ é um coproduto. Em particular, dois objetos $X$ e $Y$ tem um produto em $\mathcal{C}$ se, e somente se, tem um coproduto em $\mathcal{C}$.

Demonstração. [Lan98], Capítulo VIII, Teorema 2, pág. 190.

Seja $\mathcal{C}$ uma Ab-categoria e $X, Y, Z \in \mathcal{C}$. Então a composição o $: \operatorname{Hom}_{\mathcal{C}}(Y, Z) \times \operatorname{Hom}_{\mathcal{C}}(X, Y)$ $\rightarrow \operatorname{Hom}_{\mathcal{C}}(X, Z)$, tal que $(g, f) \mapsto g \circ f$, é bilinear. Usando a propriedade universal do produto tensorial para $\mathbb{Z}$-módulos $\otimes_{\mathbb{Z}}$ (veja Seção B.3, Subseção B.3.7), temos que existe uma única aplicação linear $\operatorname{Hom}_{\mathcal{C}}(Y, Z) \otimes_{\mathbb{Z}} \operatorname{Hom}_{\mathcal{C}}(X, Y) \rightarrow \operatorname{Hom}_{\mathcal{C}}(X, Z)$. Portanto para cada tripla $X, Y, Z \in \mathcal{C}$, existe um morfismo de grupos abelianos aditivos chamado composição tal que, $g \otimes f \mapsto g \circ f$.

Definição B.2.5. Sejam $\mathcal{C}$ e $\mathcal{D}$ Ab-categorias. Um funtor $F: \mathcal{C} \rightarrow \mathcal{D}$ é dito ser aditivo quando toda aplicação $T: \operatorname{Hom}_{\mathcal{C}}(X, Y) \rightarrow \operatorname{Hom}_{\mathcal{D}}(T(X), T(Y))$ é um homomorfismo de grupos abelianos aditivos, isto é, $T(f+g)=T(f)+T(g)$, para todos $f, g \in \operatorname{Hom}_{\mathcal{C}}(X, Y)$.

Claramente, a composição de funtores aditivos é um funtor aditivo. 
Definição B.2.6 (Categoria Aditiva). Uma categoria $\mathcal{C}$ é aditiva se:

1. $\mathcal{C}$ tem um objeto zero 0. Como o objeto 0 é inicial e terminal, existe uma única aplicação nula entre dois objetos $X$ e $Y$, via $X \rightarrow 0 \rightarrow Y$;

2. $\mathcal{C}$ é uma Ab-categoria, isto é, $H_{0} m_{\mathcal{C}}(X, Y)$ é um grupo abeliano aditivo para todos $X, Y$ $\in \mathcal{C}$, tal que a aplicação nula é o elemento neutro e a composição é bilinear;

3. $\mathcal{C}$ tem um biproduto para cada par de objetos.

Antes de definir uma Categoria Abeliana, precisamos definir mais alguns conceitos:

Definição B.2.7 (kernel/cokernel). Seja $\mathcal{C}$ uma categoria com morfismos nulos e sejam $X, Y \in \mathcal{C}$. Seja $f \in \operatorname{Hom}_{\mathcal{C}}(X, Y)$.

1. O kernel de $f$ é um par $(K, k)$ em que $K \in \mathcal{C}$ e $k: K \rightarrow X$ é tal que $f \circ k=0$ e se existe um $g \in \operatorname{Hom}_{\mathcal{C}}(P, X)$ tal que $f \circ g=0$, existe um único $h \in H_{\text {or }}(P, K)$ tal que $g=k \circ h$. Isto é,

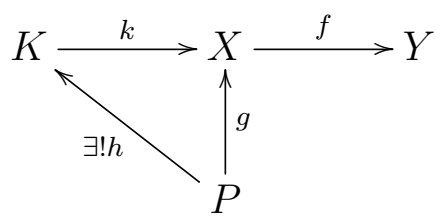

2. $O$ cokernel de $f$ é um par $(C, c)$ em que $C \in \mathcal{C}$ e $c: Y \rightarrow C$ é tal que $c \circ f=0$ e se existe um $q \in \operatorname{Hom}_{\mathcal{C}}(Y, Q)$ tal que $q \circ f=0$, existe um único $d \in H_{\mathcal{C}}(C, Q)$ tal que $d \circ c=q$. Isto é,

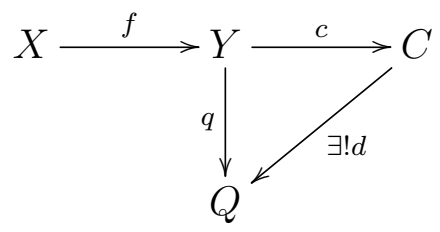

Sejam $\mathcal{C}$ uma categoria, $X, Y \in \mathcal{C}$ e $f \in \operatorname{Hom}_{\mathcal{C}}(X, Y)$. Sejam $(K, k)$ e $(C, c)$, o kernel e o cokernel de $f$, respectivamente. Então a imagem de $f$ é o kernel do seu cokernel, ou seja, $i m(f)=k e r(c)$ e a coimagem de $f$ é o cokernel do seu kernel, ou seja, $\operatorname{coim}(f)=\operatorname{coker}(k)$. Pela definição kernels são sempre monomorfismos e cokernels são sempre epimorfismos.

Definição B.2.8 (Categoria Abeliana). C é uma categoria abeliana se satisfaz as seguintes condições:

1. $\mathcal{C}$ é uma categoria aditiva;

2. Todo morfismo em $\mathcal{C}$ tem um kernel e um cokernel;

3. Todo monomorfismo é um kernel e todo epimorfismo é um cokernel.

A condição 3. é poderosa. Ela implica, por exemplo, que qualquer morfismo $f$ que seja monomorfismo e epimorfismo é um isomorfismo. Para $f: X \rightarrow Y$ monomorfismo, significa que $f=\operatorname{ker}(g)$, para algum $g: Y \rightarrow Z$, daí $g \circ f=0=0 \circ f$. Como $f$ é epimorfismo, temos que $g=0: Y \rightarrow Z$. Então $f=k e r(g)=k e r(0)$. Temos que $1_{Y}: Y \rightarrow Y$ é o $k e r(0)$, pois $0 \circ 1_{Y}=0$. Portanto $f=1_{Y}$ é um isomorfismo. As categorias $R$-mod, mod- $R$, AbGroups (e muitas outras) são todas categorias abelianas, com os kernels e cokernels usuais.

Em [Eti11] a definição de uma categoria abeliana, na verdade é o Teorema de FreydMitchell: 
Definição B.2.9. Uma categoria abeliana é uma Ab-categoria que é equivalente à uma subcategoria plena $\mathcal{C}$ da categoria $R$-mod, fechada sob somas diretas finitas, kernels, cokernels e imagens de morfismos.

Proposição B.2.4. Em uma categoria abeliana $\mathcal{C}$, todo morfismo $f$ tem uma fatorização $f=v \circ u$, em que $v$ é um monomorfismo e u é um epimorfismo.

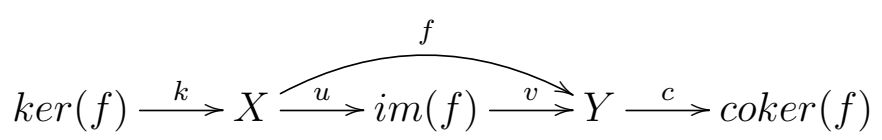

Além disso, $v=\operatorname{ker}(\operatorname{coker}(f))$ e $u=\operatorname{coker}(k e r(f))$, ou seja, c $\circ v=0$ e $u \circ k=0$.

Demonstração. [Lan98], Capítulo VIII, Proposição 1, pág. 195.

\section{B.3 Categorias R-mod e mod-R}

A categoria $R$-mod tem como objetos $R$-módulos à esquerda, tal que $R$ é um anel. Os morfismos dessa categoria são os homomorfismos de $R$-módulos à esquerda. Dados $M, M^{\prime} \in$ $R$-mod, todos os $R$-homomorfismos de $M$ para $M^{\prime}$ formam um grupo abeliano aditivo, denotado por $\operatorname{Hom}_{R-\bmod }\left(M, M^{\prime}\right)$ ou simplesmente $\operatorname{Hom}_{R}\left(M, M^{\prime}\right)$. A adição em $\operatorname{Hom}_{R}\left(M, M^{\prime}\right)$ é definida por $(f+g)(a)=f(a)+g(a)$.

Vimos que a categoria $R$-mod é uma Ab-categoria, aditiva e abeliana. Vimos também que em $R$-mod a soma direta entre dois $R$-módulos sempre existe. Na teoria clássica dos módulos soma direta finita e produto direto finito são indistinguíveis.

Também existe a categoria dos $R$-módulos à direita mod- $R$. Vou me referir à um $R$ módulo à esquerda somente como $R$-módulo. Somente vou especificar "módulo à esquerda" e "módulo à direita" quando for necessário.

\section{B.3.1 Funtores covariantes de R-mod em S-mod}

Sejam $R, S$ dois anéis. Suponha que cada módulo $M$ em $R$-mod está associado com um módulo $F(M)$ em $S$-mod e que cada $R$-homomorfismo $f: M \rightarrow M^{\prime}$ corresponde à um $S$-homomorfismo $F(f): F(M) \rightarrow F\left(M^{\prime}\right)$. Além disso suponha que:

1. $F\left(i d_{M}\right)=i d_{F(M)}$, para todo $M \in R$-mod;

2. $F(g \circ f)=F(g) \circ F(f)$, para todos $f: M \rightarrow M^{\prime}, g: M^{\prime} \rightarrow M^{\prime \prime}$ em $R$-mod.

Nestas circunstâncias dizemos que temos um funtor covariante $F: R$-mod $\rightarrow S$-mod, de $R$-módulos para $S$-módulos.

Se temos um diagrama comutativo de $R$-módulos e $R$-homomorfismos, quando aplicamos o funtor covariante $F$, obtemos um diagrama em $S$-mod, que também é comutativo. Se $f: M \rightarrow M^{\prime}$ é um isomorfismo em $R$-mod e $g: M^{\prime} \rightarrow M$ é seu inverso, então $F(f)$ : $F(M) \rightarrow F\left(M^{\prime}\right)$ é um isomorfismo em $S$-mod e $F(g): F\left(M^{\prime}\right) \rightarrow F(M)$ é seu inverso.

Definição B.3.1. Seja $F: R$-mod $\rightarrow S$-mod um funtor covariante. $F$ é dito aditivo se para $f: M \rightarrow M^{\prime}$ e $g: M \rightarrow M^{\prime}, R$-homomorfismos, $F(f+g)=F(f)+F(g)$.

Definição B.3.2. Uma sequência de R-homomorfismos em $R$-mod

$$
\ldots \stackrel{f_{n-1}}{\longrightarrow} M_{n-1} \stackrel{f_{n}}{\longrightarrow} M_{n} \stackrel{f_{n+1}}{\longrightarrow} M_{n+1} \stackrel{f_{n+2}}{\longrightarrow} \ldots
$$


é exata em $M_{n}$ se $i m\left(f_{n}\right)=k e r\left(f_{n+1}\right)$. Dizemos que a sequência é exata se for exata em todos os $R$-módulos que a compõem, exceto eventualmente nas extremidades.

Teorema B.3.1. Seja $0 \longrightarrow M_{1} \stackrel{\sigma_{1}}{\rightarrow} M \stackrel{\pi_{2}}{\longrightarrow} M_{2} \rightarrow 0$ uma sequência exata em $R$-mod. As seguintes afirmações são equivalentes:

1. $\operatorname{im}\left(\sigma_{1}\right)\left(=k e r\left(\pi_{2}\right)\right)$ é um somando direto de $M$.

2. Existe um R-homomorfismo $\pi_{1}: M \rightarrow M_{1}$ tal que $\pi_{1} \circ \sigma_{1}=1_{M_{1}}$.

3. Existe um R-homomorfismo $\sigma_{2}: M_{2} \rightarrow M$ tal que $\pi_{2} \circ \sigma_{2}=1_{M_{2}}$.

4. Existem R-homomorfismos $\pi_{1}: M \rightarrow M_{1}$ e $\sigma_{2}: M_{2} \rightarrow M$ tal que $M=M_{1} \oplus M_{2}$.

Demonstração. [Nor73], Capítulo 1, Teorema 4, pág. 5.

Definição B.3.3. Seja $0 \longrightarrow M_{1} \stackrel{\sigma_{1}}{\rightarrow} M \stackrel{\pi_{2}}{\rightarrow} M_{2} \rightarrow 0$ uma sequência exata em $R$-mod. Se uma das condições do Teorema B.3.1 for satisfeita, dizemos que a sequência exata cinde.

Por outro lado, se $0 \longrightarrow M^{\prime} \longrightarrow M \longrightarrow M^{\prime \prime} \longrightarrow 0$ é uma sequência exata que cinde, então temos um isomorfismo $M \cong M^{\prime} \oplus M^{\prime \prime}$.

Teorema B.3.2. Seja $0 \longrightarrow M_{1} \stackrel{\sigma_{1}}{\longrightarrow} M \stackrel{\pi_{2}}{\longrightarrow} M_{2} \rightarrow 0$ uma sequência exata que cinde em $R$ $\bmod$ e $F: R$ - $\bmod \rightarrow S$-mod um funtor covariante aditivo. Então:

$$
0 \longrightarrow F\left(M_{1}\right) \stackrel{F\left(\sigma_{1}\right)}{\longrightarrow} F(M) \stackrel{F\left(\pi_{2}\right)}{\longrightarrow} F\left(M_{2}\right) \longrightarrow 0
$$

é uma sequência exata que cinde em $S$-mod.

Demonstração. [Nor73], Capítulo 1, Teorema 5, pág. 6.

Definição B.3.4. Seja $F: R$-mod $\rightarrow S$-mod um funtor covariante. Assuma que sempre que $0 \rightarrow M^{\prime} \rightarrow M \rightarrow M^{\prime \prime} \rightarrow 0$ é exata em $R$-mod, então $0 \rightarrow F\left(M^{\prime}\right) \rightarrow F(M) \rightarrow F\left(M^{\prime \prime}\right)$ (respect. $F\left(M^{\prime}\right) \rightarrow F(M) \rightarrow F\left(M^{\prime \prime}\right) \rightarrow 0$ ) é exata em $S$-mod. Dizemos que $F$ é exato à esquerda (respect. exato à direita). Caso a exatidão de $0 \rightarrow M^{\prime} \rightarrow M \rightarrow M^{\prime \prime} \rightarrow 0$ implique que $F\left(M^{\prime}\right) \rightarrow F(M) \rightarrow F\left(M^{\prime \prime}\right)$ é exata em $S$-mod, dizemos que $F$ é parcialmente exato. Se $F$ é exato à esquerda e à direita, isto é, sempre que $0 \rightarrow M^{\prime} \rightarrow M \rightarrow M^{\prime \prime} \rightarrow 0$ é exata em $R$-mod implica que $0 \rightarrow F\left(M^{\prime}\right) \rightarrow F(M) \rightarrow F\left(M^{\prime \prime}\right) \rightarrow 0$ é exata em S-mod, então $F$ é dito ser um funtor exato.

Então, pela Definição B.3.4, percebemos que se $F$ for exato à esquerda preserva monomorfismos e se for exato à direita preserva epimorfismos.

Seja $F: R$-mod $\rightarrow S$-mod um funtor covariante:

Lema B.3.1. Suponha que $F$ é exato à esquerda e que $0 \rightarrow M^{\prime} \rightarrow M \rightarrow M^{\prime \prime}$ é exata em $R$-mod. Então $0 \rightarrow F\left(M^{\prime}\right) \rightarrow F(M) \rightarrow F\left(M^{\prime \prime}\right)$ é exata em $S$-mod.

Lema B.3.2. Suponha que $F$ é exato à direita e que $M^{\prime} \rightarrow M \rightarrow M^{\prime \prime} \rightarrow 0$ é exata em $R$-mod. Então $F\left(M^{\prime}\right) \rightarrow F(M) \rightarrow F\left(M^{\prime \prime}\right) \rightarrow 0$ é exata em $S$-mod.

Lema B.3.3. Suponha que $F$ é exato e que $M^{\prime} \rightarrow M \rightarrow M^{\prime \prime}$ é exata em $R$-mod. Então $F\left(M^{\prime}\right) \longrightarrow F(M) \rightarrow F\left(M^{\prime \prime}\right)$ é exata em $S$-mod. 
As provas dos lemas acima são aplicações diretas da Definição B.3.4. Por esse motivo foram omitidas.

Teorema B.3.3. Suponha que F é parcialmente exato, então ele é aditivo.

Demonstração. [Nor73], Capítulo 1, Teorema 6, pág. 7.

Suponha que o funtor covariante $F: R$-mod $\rightarrow S$-mod é exato e que $M_{1}$ é um submódulo de um $R$-módulo $M$. Então a aplicação inclusão nos dá uma sequência exata $0 \rightarrow M_{1} \rightarrow M$ e então $0 \rightarrow F\left(M_{1}\right) \rightarrow F(M)$ é exata. Dessa forma $F\left(M_{1}\right)$ pode ser considerado um $S$ submódulo de $F(M)$.

Proposição B.3.1. Seja $F: R$-mod $\rightarrow S$-mod um funtor covariante exato. Então $F$ preserva imagens e kernels.

Demonstração. [Nor73], Capítulo 1, Exercício 5, pág. 18.

\section{B.3.2 Funtores contravariantes de R-mod em S-mod}

Sejam $R, S$ dois anéis. Suponha que cada módulo $M$ em $R$-mod está associado com um módulo $G(M)$ em $S$-mod e que cada $R$-homomorfismo $f: M \rightarrow M^{\prime}$ corresponde à um $S$-homomorfismo $G(f): G\left(M^{\prime}\right) \rightarrow G(M)$. Além disso suponha que:

1. $G\left(i d_{M}\right)=i d_{G(M)}$, para todo $M \in R$-mod;

2. $G(g \circ f)=G(f) \circ G(g)$, para todos $f: M \rightarrow M^{\prime}, g: M^{\prime} \rightarrow M^{\prime \prime}$ em $R$-mod.

Dizemos que temos um funtor contravariante $G: R$-mod $\rightarrow S$-mod, de $R$-módulos para $S$-módulos.

Se temos um diagrama comutativo de $R$-módulos e $R$-homomorfismos, quando aplicamos o funtor contravariante $G$, obtemos um diagrama em $S$-mod, que também é comutativo, mas com todas as "flechas" invertidas. Se $f: M \rightarrow M^{\prime}$ é um isomorfismo em $R$-mod e $g: M^{\prime} \rightarrow M$ é seu inverso, então $G(f): G\left(M^{\prime}\right) \rightarrow G(M)$ é um isomorfismo em $S$-mod e $G(g): G(M) \rightarrow G\left(M^{\prime}\right)$ é seu inverso.

O funtor contravariante aditivo é definido de maneira análoga ao funtor covariante aditivo.

Teorema B.3.4. Seja $0 \longrightarrow M_{1} \stackrel{\sigma_{1}}{\longrightarrow} M \stackrel{\pi_{2}}{\longrightarrow} M_{2} \longrightarrow 0$ uma sequência exata que cinde em $R$ $\bmod e G: R-\bmod \rightarrow S-\bmod$ um funtor contravariante aditivo. Então:

$$
0 \longrightarrow G\left(M_{2}\right) \stackrel{G\left(\pi_{2}\right)}{\longrightarrow} G(M) \stackrel{G\left(\sigma_{1}\right)}{\longrightarrow} G\left(M_{1}\right) \longrightarrow 0
$$

é uma sequência exata que cinde em $S$-mod.

Demonstração. A prova é análoga à demonstração do Teorema B.3.2.

Definição B.3.5. Seja $G: R$-mod $\rightarrow S$-mod um funtor contravariante. É dito ser exato à esquerda (respect. exato à direita) se a exatidão de $0 \rightarrow M^{\prime} \rightarrow M \rightarrow M^{\prime \prime} \rightarrow 0$ em $R$-mod $i m$ plica na exatidão de $0 \rightarrow G\left(M^{\prime \prime}\right) \rightarrow G(M) \rightarrow G\left(M^{\prime}\right)$ (respect. $G\left(M^{\prime \prime}\right) \rightarrow G(M) \rightarrow G\left(M^{\prime}\right) \rightarrow 0$ ) em S-mod. Além disso, caso a exatidão de $0 \rightarrow M^{\prime} \rightarrow M \rightarrow M^{\prime \prime} \rightarrow 0$ implique na exatidão de $G\left(M^{\prime \prime}\right) \rightarrow G(M) \rightarrow G\left(M^{\prime}\right)$ em $S$-mod, dizemos que $G$ é parcialmente exato. Finalmente, se $G$ é exato à esquerda e à direita, isto é, a exatidão de $0 \rightarrow M^{\prime} \rightarrow M \rightarrow M^{\prime \prime} \rightarrow 0$ em $R$ $\bmod$ implica na exatidão de $0 \rightarrow G\left(M^{\prime \prime}\right) \rightarrow G(M) \rightarrow G\left(M^{\prime}\right) \rightarrow 0$ em $S$-mod, então $G$ é dito ser um funtor exato. 
Então um funtor contravariante exato à esquerda converte um epimorfismo em um monomorfismo, enquanto que um funtor contravariante exato à direita transforma um monomorfismo em um epimorfismo.

Seja $G: R$-mod $\rightarrow S$-mod um funtor contravariante:

Lema B.3.4. Suponha que $G$ é exato à esquerda e que $M^{\prime} \rightarrow M \rightarrow M^{\prime \prime} \rightarrow 0$ é exata em $R$-mod. Então $0 \longrightarrow G\left(M^{\prime \prime}\right) \rightarrow G(M) \rightarrow G\left(M^{\prime}\right)$ é exata em $S$-mod.

Lema B.3.5. Suponha que $G$ é exato à direita e que $0 \rightarrow M^{\prime} \rightarrow M \rightarrow M^{\prime \prime}$ é exata em $R$-mod. Então $G\left(M^{\prime \prime}\right) \rightarrow G(M) \rightarrow G\left(M^{\prime}\right) \rightarrow 0$ é exata em $S$-mod.

Lema B.3.6. Suponha que $G$ é exato e que $M^{\prime} \rightarrow M \rightarrow M^{\prime \prime}$ é exata em $R$-mod. Então $G\left(M^{\prime \prime}\right) \longrightarrow G(M) \longrightarrow G\left(M^{\prime}\right)$ é exata em $S$-mod.

As provas dos lemas acima, seguem diretamente da Definição B.3.5.

Teorema B.3.5. Suponha que $G$ é parcialmente exato, então ele é aditivo.

Demonstração. A prova é similar à demonstração do Teorema B.3.3

\section{B.3.3 Bifuntores}

Ao invés de considerar funtores em uma variável, podemos considerar funtores em várias variáveis. Um tal funtor pode ser covariante em algumas das variáveis e contravariante nas outras. Vamos definir um funtor de duas variáveis, um bifuntor, contravariante na primeira coordenada e covariante na segunda.

Sejam $R, R^{\prime}$ e $S$ anéis. Suponha que para cada $M \in R$-mod e $N \in R^{\prime}$-mod existe um módulo $T(M, N)$ em $S$-mod associado, e suponha que dados $f: M^{\prime} \rightarrow M$ em $R$-mod e $g: N \rightarrow N^{\prime}$ em $R^{\prime}$-mod, existe um $S$-homomorfismo correspondente $T(f, g): T(M, N) \rightarrow$ $T\left(M^{\prime}, N^{\prime}\right)$. Se ainda:

1. $T\left(i d_{M}, i d_{N}\right)=i d_{T(M, N)}$;

2. $T\left(f \circ f_{1}, g_{1} \circ g\right)=T\left(f_{1}, g_{1}\right) \circ T(f, g)$, para todas $M^{\prime \prime} \stackrel{f_{1}}{\rightarrow} M^{\prime} \stackrel{f}{\rightarrow} M$ em $R$-mod e $N \stackrel{g}{\rightarrow} N^{\prime} \stackrel{g_{1}}{\longrightarrow} N^{\prime \prime}$ em $R^{\prime}$-mod.

então dizemos que $T$ é um bifuntor de $R$-mod $\times R^{\prime}$-mod em $S$-mod, que é contravariante na primeira variável e covariante na segunda.

Assuma que $T$ é tal funtor. Tome um módulo fixo $M$ em $R$-mod. Então obtemos um funtor covariante $T(M, \cdot): R^{\prime}$-mod $\rightarrow S$-mod. Seja $g: N \rightarrow N^{\prime}$ em $R^{\prime}$-mod, então $T(M, g)$ significa $T\left(i d_{M}, g\right)$. Se tomarmos $N$ fixo em $R^{\prime}$-mod, obtemos um funtor contravariante $T(\cdot, N): R$-mod $\rightarrow S$-mod.

Se $T(M, \cdot)$ e $T(\cdot, N)$ são aditivos, para todos $M, N$, então $T$ é dito ser aditivo. Se $T$ for aditivo, então:

$$
T\left(M, g_{1}+g_{2}\right)=T\left(M, g_{1}\right)+T\left(M, g_{2}\right)
$$

em que $g_{1}, g_{2}: N \rightarrow N^{\prime}$ em $R^{\prime}$-mod, e:

$$
T\left(f_{1}+f_{2}, N\right)=T\left(f_{1}, N\right)+T\left(f_{2}, N\right)
$$

em que $f_{1}, f_{2}: M^{\prime} \rightarrow M$ em $R$-mod. 
Ainda, se $T(M, \cdot)$ e $T(\cdot, N)$ são exatos à esquerda, para todos $M, N$, então $T$ é chamado de exato à esquerda. Se $T$ é exato à esquerda e as sequências:

$$
M^{\prime} \rightarrow M \rightarrow M^{\prime \prime} \rightarrow 0
$$

$\mathrm{e}$

$$
0 \rightarrow N^{\prime} \longrightarrow N \longrightarrow N^{\prime \prime}
$$

são exatas em $R$-mod e $R^{\prime}$-mod respectivamente, então as sequências:

$$
0 \longrightarrow T\left(M^{\prime \prime}, N\right) \rightarrow T(M, N) \rightarrow T\left(M^{\prime}, N\right)
$$

e

$$
0 \longrightarrow T\left(M, N^{\prime}\right) \longrightarrow T(M, N) \rightarrow T\left(M, N^{\prime \prime}\right)
$$

serão exatas em $S$-mod. As noções de funtores exato à direita, exato e parcialmente exato são generalizadas da mesma maneira.

\section{B.3.4 O funtor Hom}

Sejam $M, N \in R$-mod. Devemos lembrar que todos os $R$-homomorfismos de $M$ para $N$ formam um grupo abeliano aditivo denotado por $\operatorname{Hom}_{R}(M, N)$. Observamos também que a categoria dos grupos abelianos aditivos é identificada com a categoria dos $\mathbb{Z}$-módulos.

Sejam $u: M^{\prime} \rightarrow M$ e $v: N \rightarrow N^{\prime}, R$-homomorfismos. Definimos a aplicação:

$$
\operatorname{Hom}_{R}(u, v): \operatorname{Hom}_{R}(M, N) \rightarrow \operatorname{Hom}_{R}\left(M^{\prime}, N^{\prime}\right)
$$

tal que $f \in \operatorname{Hom}_{R}(M, N) \mapsto v \circ f \circ u \in \operatorname{Hom}_{R}\left(M^{\prime}, N^{\prime}\right)$. Claramente $\operatorname{Hom}_{R}(u, v)$ é um homomorfismo de grupos abelianos e se $u, v$ são aplicações identidade, então $\operatorname{Hom}_{R}(u, v)$ é também uma aplicação identidade. Além disso, se $u^{\prime}: M^{\prime \prime} \rightarrow M^{\prime}$ e $v^{\prime}: N^{\prime} \rightarrow N^{\prime \prime}$ estão em $R$-mod, então:

$$
\operatorname{Hom}_{R}\left(u \circ u^{\prime}, v^{\prime} \circ v\right)=\operatorname{Hom}_{R}\left(u^{\prime}, v^{\prime}\right) \circ \operatorname{Hom}_{R}(u, v)
$$

De fato, $\operatorname{Hom}_{R}(M, N)$ é um bifuntor de $R$ - $\bmod \times R$-mod em $\mathbb{Z}-\bmod$ (categoria dos grupos abelianos aditivos) e é contravariante na primeira variável e covariante na segunda, isto é, para $M \in R$-mod fixo, $\operatorname{Hom}_{R}(M, \cdot)$ é covariante, e para $N \in R$-mod fixo, $\operatorname{Hom}_{R}(\cdot, N)$ é contravariante. Este funtor é chamado de funtor Hom.

Notamos que se $u_{1}, u_{2}: M^{\prime} \rightarrow M$ e $v_{1}, v_{2}: N \rightarrow N^{\prime}$, então:

$$
\operatorname{Hom}_{R}\left(u_{1}+u_{2}, N\right)=\operatorname{Hom}_{R}\left(u_{1}, N\right)+\operatorname{Hom}_{R}\left(u_{2}, N\right)
$$

e

$$
\operatorname{Hom}_{R}\left(M, v_{1}+v_{2}\right)=\operatorname{Hom}_{R}\left(M, v_{1}\right)+\operatorname{Hom}_{R}\left(M, v_{2}\right)
$$

Concluímos que o funtor Hom é aditivo.

Teorema B.3.6. O funtor Hom é exato à esquerda.

Demonstração. [Nor73], Capítulo 2, Teorema 1, pág. 38.

O Teorema B.3.6 diz que se $0 \longrightarrow M^{\prime} \rightarrow M \longrightarrow M^{\prime \prime} \rightarrow 0$ é exata em $R$-mod, então

$$
0 \rightarrow \operatorname{Hom}_{R}\left(M^{\prime \prime}, N\right) \rightarrow \operatorname{Hom}_{R}(M, N) \rightarrow \operatorname{Hom}_{R}\left(M^{\prime}, N\right)
$$


é exata para todo $N \in R$-mod. Temos também que se $0 \rightarrow N^{\prime} \rightarrow N \rightarrow N^{\prime \prime} \rightarrow 0$ é exata em $R$-mod, então

$$
0 \rightarrow \operatorname{Hom}_{R}\left(M, N^{\prime}\right) \rightarrow \operatorname{Hom}_{R}(M, N) \rightarrow \operatorname{Hom}_{R}\left(M, N^{\prime \prime}\right)
$$

é exata para todo $M \in R$-mod.

\section{B.3.5 Módulos Projetivos}

Seja $P$ um objeto de $R$-mod.

Definição B.3.6. $P$ é dito ser um $R$-módulo projetivo se o funtor $\operatorname{Hom}_{R}(P, \cdot)$, de $R$-mod em $\mathbb{Z}$-mod, é exato.

Na verdade, em alguns livros existe a seguinte proposição:

Proposição B.3.2. Um $R$-módulo à esquerda $P$ é projetivo se, e somente se, $\operatorname{Hom}_{R}(P, \cdot)$ é um funtor exato.

Demonstração. [Rot09], Capítulo 3, Proposição 3.2, pág. 100.

Se um módulo é projetivo, então qualquer módulo que seja isomorfo à ele também é. O seguinte lema nos fornece um critério útil para saber se um módulo é projetivo:

Lema B.3.7. Seja $P$ um R-módulo. Então as seguintes afirmações são equivalentes:

1. P é projetivo.

2. Para todo $M, N \in R$-mod, tais que existe um epimorfismo $g: M \rightarrow N$ e para todo $f: P \rightarrow N$, R-homomorfismo, existe $f^{\prime}: P \rightarrow M, R$-homomorfismo, tal que $g \circ f^{\prime}=f$, ou seja,

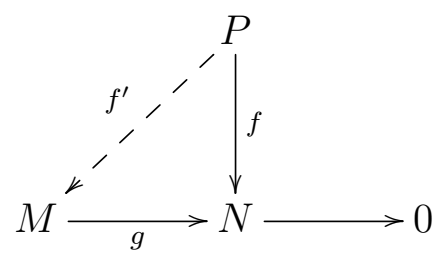

Demonstração. [Nor73], Capítulo 2, Lema 1, pág. 27.

Definição B.3.7. Se $M$ é um $R$-módulo que possui uma base, isto é, um sistema de geradores linearmente independentes, $M$ é um $R$-módulo livre. Equivalentemente, $M$ é um $R$-módulo livre se é isomorfo à uma soma direta de cópias de $R$, ou seja, $M \cong \oplus_{i \in I} R$.

Teorema B.3.7. Todo R-módulo livre é R-projetivo.

Demonstração. [Nor73], Capítulo 2, Teorema 3, pág. 40.

A recíproca do Teorema B.3.7 não é verdadeira, ou seja, ser $R$-projetivo não implica ser $R$-livre:

Exemplo B.3.1. Seja $R=M_{n \times n}(k)$, a álgebra das matrizes com $n$ linhas e $n$ colunas com entradas em um corpo $k, n \geq 2$. O R-módulo $M=\left\{\left(\begin{array}{c}a_{1} \\ \vdots \\ a_{n}\end{array}\right) \mid a_{i} \in k\right\}$ é projetivo, mas não é livre. 
Definição B.3.8. Sejam $M, N R$-módulos. Direi que $N$ é imagem homomórfica de $M$ se existir um epimorfismo $\varphi: M \rightarrow N$.

Proposição B.3.3. Seja $M$ um R-módulo. Existe um R-módulo livre $F$ tal que $M$ é imagem homomórfica de $F$, isto é, existe $\pi: F \rightarrow M$ epimorfismo. Em outras palavras, $M$ é isomorfo à um quociente do R-módulo livre $F$.

Demonstração. [Rot03], Capítulo 7, Proposição 7.51, pág. 473.

Teorema B.3.8. Seja $P$ um R-módulo. Então $P$ é um $R$-módulo projetivo se, e somente se, toda sequência exata da forma $0 \rightarrow M \stackrel{f}{\rightarrow} N \stackrel{g}{\rightarrow} P \longrightarrow 0$ cinde.

Demonstração. [Rot03], Capítulo 7, Proposição 7.54, pág. 475. A prova usa pullback como ferramenta.

Teorema B.3.9. Seja $P$ um R-módulo. Então $P$ é um $R$-módulo projetivo se, e somente se, $P$ é isomorfo à um somando direto de um $R$-módulo livre.

Demonstração. [Nor73], Capítulo 2, Teorema 7, pág. 28 ou [Rot03], Capítulo 7, Teorema 7.56, pág. 476.

Teorema B.3.10. Seja $\left(P_{i}\right)_{i \in I}$ uma família de $R$-módulos arbitrários e seja $P=\oplus_{i \in I} P_{i}$. Então $P$ é $R$-projetivo se, e somente se, $P_{i}$ é $R$-projetivo, para cada $i \in I$.

Demonstração. [Nor73], Capítulo 2, Teorema 4, pág. 41.

Corolário B.3.1. Todo somando direto de um R-módulo projetivo é R-projetivo.

Teorema B.3.11. Seja $M$ um $R$-módulo. Então existe uma sequência exata $P \rightarrow M \rightarrow 0$ em R-mod, tal que $P$ é R-projetivo.

Demonstração. [Nor73], Capítulo 2, Teorema 6, pág. 28.

Teorema B.3.12 (Teorema da Base Dual). Um R-módulo P é projetivo se, e somente se, existem familias $\left\{a_{i}\right\}_{i \in I}$ de elementos de $P$ e $\left\{f_{i}\right\}_{i \in I}$ de elementos de $\operatorname{Hom}_{R}(P, R)$ tal que:

1. Para cada $a \in P, f_{i}(a)=0$, para quase todos $i$;

2. $a=\sum f_{i}(a) a_{i}$, para cada $a \in P$.

Quando $P$ é projetivo, a família $\left\{a_{i}\right\}_{i \in I}$ pode ser tomada como um sistema de geradores de $P$.

Demonstração. [Nor73], Capítulo 2, Teorema 8, pág. 29.

Proposição B.3.4 (Lema de Schanuel). Considere as duas sequências exatas:

$$
0 \longrightarrow K \stackrel{i}{\longrightarrow} P \stackrel{\pi}{\longrightarrow} M \longrightarrow 0
$$

$e$

$$
0 \longrightarrow K^{\prime} \stackrel{i^{\prime}}{\longrightarrow} P^{\prime} \stackrel{\pi^{\prime}}{\longrightarrow} M \longrightarrow 0
$$

tais que $P$ e $P^{\prime}$ são $R$-projetivos. Então existe um isomorfismo $K \oplus P^{\prime} \cong K^{\prime} \oplus P$.

Demonstração. [Rot03], Capítulo 7, Proposição 7.60, pág. 479. 


\section{B.3.6 Módulos Injetivos}

Seja $E$ um objeto de $R$-mod.

Definição B.3.9. $E$ é dito ser um $R$-módulo injetivo se o funtor $\operatorname{Hom}_{R}(\cdot, E)$, de $R$-mod em $\mathbb{Z}$-mod, é exato.

Claramente, todo $R$-módulo que é isomorfo à um módulo injetivo é também injetivo.

Lema B.3.8. Seja E um R-módulo. Então as seguintes afirmações são equivalentes:

1. E é injetivo.

2. Para todo $M, N \in R$-mod, tais que existe um monomorfismo $i: M \rightarrow N$ e para todo $f: M \rightarrow E, R$-homomorfismo, existe $f^{\prime}: N \rightarrow E, R$-homomorfismo, tal que $f^{\prime} \circ i=f$, ou seja,

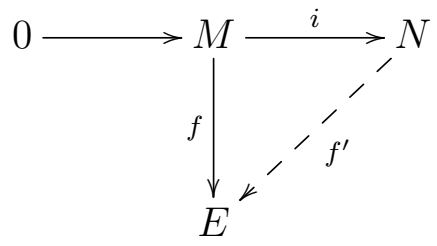

Demonstração. [Rot03], Capítulo 7, Proposição 7.63, pág. 481.

Teorema B.3.13. Um R-módulo E é injetivo se, e somente se, toda sequência exata da forma $0 \rightarrow E \stackrel{f}{\rightarrow} M \stackrel{g}{\rightarrow} N \rightarrow 0$ cinde.

Demonstração. [Rot03], Capítulo 7, Proposição 7.64, pág. 481. A prova usa pushout como ferramenta.

Corolário B.3.2. Se um R-módulo E é injetivo e é R-submódulo de um $R$-módulo $M$, então $E$ é somando direto de $M$.

Teorema B.3.14. Seja $\left(E_{i}\right)_{i \in I}$ uma família de $R$-módulos arbitrários e $E=\prod_{i \in I} E_{i}$ (produto direto). Então $E$ é injetivo se, e somente se, $E_{i}$ é injetivo, para cada $i \in I$.

Demonstração. [Nor73], Capítulo 2, Teorema 9, pág. 45 ou [Rot03], Capítulo 7, Proposição 7.66, pág. 482.

Corolário B.3.3. Todo somando direto de um R-módulo injetivo é injetivo.

Teorema B.3.15 (Critério de Baer). Um R-módulo E é injetivo se, e somente se, toda aplicação $f: I \rightarrow E$, tal que $I$ é um ideal à esquerda de $R$, pode ser estendida à $R$.

Demonstração. [Rot03], Capítulo 7, Teorema 7.68, pág. 483.

Teorema B.3.16. Seja $M$ um R-módulo. Podemos construir um sequência exata $0 \rightarrow M \rightarrow E$ em $R$-mod, tal que E é R-injetivo.

Esse resultado acima mostra que todo $R$-módulo $M$ pode ser imerso em um módulo injetivo $E$. Isto é, existe um monomorfismo de $M$ em $E$.

Demonstração. [Nor73], Capítulo 2, Teorema 14, pág. 34.

Lema B.3.9 (Lema de Schanuel II). Considere as duas sequências exatas:

$$
0 \longrightarrow N \stackrel{i}{\longrightarrow} E \stackrel{\pi}{\longrightarrow} M \longrightarrow 0
$$

$e$

$$
0 \longrightarrow N \stackrel{i^{\prime}}{\longrightarrow} E^{\prime} \stackrel{\pi^{\prime}}{\longrightarrow} M^{\prime} \longrightarrow 0
$$

tais que $E$ e $E^{\prime}$ são $R$-injetivos. Então existe um isomorfismo $E^{\prime} \oplus M \cong E \oplus M^{\prime}$. 


\section{B.3.7 O funtor Tensor}

Definição B.3.10. Sejam $R$ um anel, $M$ um $R$-módulo à direita e $N$ um R-módulo à esquerda. Um produto balanceado de $M$ e $N$ é um par ordenado $(P, f)$, tal que $P$ é um grupo abeliano aditivo e $f: M \times N \rightarrow P$ é uma função satisfazendo:

1. $f\left(x+x^{\prime}, y\right)=f(x, y)+f\left(x^{\prime}, y\right)$;

2. $f\left(x, y+y^{\prime}\right)=f(x, y)+f\left(x, y^{\prime}\right)$;

3. $f(x a, y)=f(x, a y)$.

para todos $x, x^{\prime} \in M, y, y^{\prime} \in N, a \in R$.

Observação B.3.1. Se $(P, f)$ é um produto balanceado de $M_{R}$ e ${ }_{R} N$, então:

1. $f(0, y)=0=f(x, 0)$;

2. $f(-x, y)=-f(x, y)=f(x,-y)$.

para todo $x \in M$ e para todo $y \in N$.

Definição B.3.11. Um produto tensorial de $M_{R} e_{R} N$ é um produto balanceado $\left(M \otimes_{R} N, \otimes\right)$ tal que se $(P, f)$ é um produto balanceado de $M_{R}$ e ${ }_{R} N$, então existe um único homomorfismo de grupos

$\varphi: M \otimes_{R} N \rightarrow P$, tal que $\varphi(x \otimes y)=f(x, y)$, para todo $(x, y) \in M \times N$, em que $x \otimes y=$ $\otimes(x, y)$. O diagrama abaixo ilustra a definição:

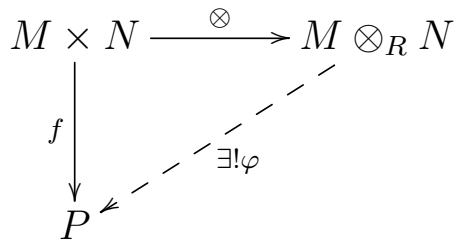

Em geral, o produto tensorial de dois módulos é somente um grupo abeliano aditivo. Se $R$ for um anel comutativo e $M, N$ forem $R$-módulos, então $M \otimes_{R} N$ será um $R$-módulo. Por exemplo, seja $R=k$ e $V, W$ dois $k$-espaços vetoriais ( $k$-módulos), então $V \otimes_{k} W$ é um $k$-espaço vetorial. A noção de bimódulos esclarece quando o produto tensorial será um $R$-módulo. Para mais detalhes indicamos [Rot09], Capítulo 2.

Proposição B.3.5. Se $\left(\left(M \otimes_{R} N\right)_{1}, \otimes_{1}\right)$ e $\left(\left(M \otimes_{R} N\right)_{2}, \otimes_{2}\right)$ são produtos tensoriais de $M_{R}$ $e_{R} N$, então existe um único isomorfismo de grupos

$$
\varphi:\left(M \otimes_{R} N\right)_{1} \rightarrow\left(M \otimes_{R} N\right)_{2}
$$

tal que $\varphi\left(x \otimes_{1} y\right)=x \otimes_{2}$ y, para todo $x \in M_{R}$ e para todo $y \in{ }_{R} N$.

Demonstração. [Rot09], Capítulo 2, Proposição 2.44, pág. 71.

Observação B.3.2. Se $\left(M \otimes_{R} N, \otimes\right)$ é um produto tensorial de $M_{R} e_{R} N$, então o grupo abeliano $M \otimes_{R} N$ é gerado por

$$
B=\{x \otimes y \mid x \in M, y \in N\}
$$

Temos que:

$$
M \otimes_{R} N=\left\{\sum_{i=1}^{n} x_{i} \otimes y_{i} \mid n \geq 1, x_{i} \in M, y_{i} \in N\right\}
$$


Teorema B.3.17. Seja $R$ um anel e sejam $M$ um $R$-módulo à direita e $N$ um $R$-módulo à esquerda. Então existe um produto tensorial de $M$ e $N$.

Demonstração. [Rot09], Capítulo 2, Proposição 2.45, pág. 72.

Observação B.3.3. Sejam $f: M_{1} \rightarrow M_{2}$ em mod- $R$ e se $g: N_{1} \rightarrow N_{2}$ em $R$-mod, então a aplicação

$$
\begin{aligned}
\varphi: M_{1} \times N_{1} & \longrightarrow M_{2} \otimes_{R} N_{2} \\
(x, y) & \longmapsto f(x) \otimes g(y)
\end{aligned}
$$

define um produto balanceado de $M_{1}$ e $N_{1}$ e portanto, podemos definir

$$
\begin{aligned}
f \otimes g: M_{1} \otimes_{R} N_{1} & \longrightarrow M_{2} \otimes_{R} N_{2} \\
x \otimes y & \longmapsto f(x) \otimes g(y)
\end{aligned}
$$

Proposição B.3.6. Sejam $f: M \rightarrow M^{\prime}$ e $g: N \rightarrow N^{\prime}$ morfismos de $\bmod -R$ e $R$-mod, respectivamente. Então existe uma única $\mathbb{Z}$-aplicação denotada por $f \otimes g: M \otimes_{R} N \rightarrow$ $M^{\prime} \otimes_{R} N^{\prime}, c o m f \otimes g: x \otimes y \mapsto f(x) \otimes g(y)$.

Demonstração. [Rot09], Capítulo 2, Proposição 2.46, pág. 74.

Corolário B.3.4. Dadas aplicações de $R$-módulos à direita, $M \stackrel{f}{\rightarrow} M^{\prime} \stackrel{f^{\prime}}{\rightarrow} M^{\prime \prime}$, e aplicações de $R$-módulos a esquerda, $N \stackrel{g}{\rightarrow} N^{\prime} \stackrel{g^{\prime}}{\rightarrow} N^{\prime \prime}$, então:

$$
\left(f^{\prime} \otimes g^{\prime}\right) \circ(f \otimes g)=f^{\prime} \circ f \otimes g^{\prime} \circ g
$$

Teorema B.3.18. 1. Sejam $R$ um anel e $M$ um $R$-módulo à direita. Existe um funtor covariante aditivo $M \otimes_{R}-: R$-mod $\rightarrow \mathbb{Z}$-mod, definido por $\left(M \otimes_{R}-\right)(N)=M \otimes_{R} N$, para todo $N \in R$-mod, e $\left(M \otimes_{R}-\right)(g)=M \otimes_{R} g=i d_{M} \otimes g$, em que $g: N \rightarrow N^{\prime} \dot{e}$ um morfismo em $R$-mod.

2. Similarmente, sejam $R$ um anel e $N$ um $R$-módulo à esquerda. Existe um funtor covariante aditivo $-\otimes_{R} N$ : mod- $R \rightarrow \mathbb{Z}$-mod, definido por $\left(-\otimes_{R} N\right)(M)=M \otimes_{R} N$, para todo $M \in \bmod -R$, e $\left(-\otimes_{R} N\right)(f)=f \otimes_{R} N=f \otimes i d_{N}$, em que $f: M \rightarrow M^{\prime}$ é um morfismo em mod- $R$.

Demonstração. [Rot09], Capítulo 2, Teorema 2.48, pág. 74.

Observação B.3.4. Tal produto $M \otimes_{R} g$ existe, pois se:

$$
\begin{aligned}
f: M \times N & \longrightarrow M \otimes_{R} N^{\prime} \\
(x, y) & \longmapsto x \otimes g(y)
\end{aligned}
$$

então $\left(M \otimes_{R} N^{\prime}, f\right)$ é um produto balanceado de $M_{R}$ e ${ }_{R} N$ e, portanto, induz um único homomorfismo de grupos $M \otimes_{R} g: M \otimes_{R} N \rightarrow M \otimes_{R} N^{\prime}$ tal que $\left(M \otimes_{R} g\right)(x \otimes y)=$ $i d_{M}(x) \otimes g(y)$. De maneira análoga, existe $f \otimes_{R} N$.

Corolário B.3.5. Se $f: M \rightarrow M^{\prime}$ e $g: N \rightarrow N^{\prime}$ são, respectivamente, isomorfismos em mod- $R$ e $R$-mod, então $f \otimes g: M \otimes_{R} N \rightarrow M^{\prime} \otimes_{R} N^{\prime}$ é um isomorfismo de grupos abelianos.

Demonstração. [Rot09], Capítulo 2, Corolário 2.49, pág. 75.

Teorema B.3.19. Seja $R$ um anel. Os funtores covariantes aditivos $M \otimes_{R}-e-\otimes_{R} N$ são exatos à direita. 
Demonstração. [Rot09], Capítulo 2, Teorema 2.63, pág. 84.

Definição B.3.12. Sejam $R, S$ anéis e $M$ um grupo abeliano aditivo. Então $M$ é um $(R, S)$ bimódulo, denotado por ${ }_{R} M_{S}$, se $M$ é um $R$-módulo à esquerda e um $S$-módulo à direita, e as duas multiplicações por escalares são relacionadas pela lei associativa:

$$
r(m s)=(r m) s
$$

para todos $r \in R, m \in M$ e $s \in S$.

Se $M$ é um $(R, S)$-bimódulo, podemos escrever $r m s$ sem os parênteses.

Corolário B.3.6. Seja $S$ uma k-álgebra e $R$ uma subálgebra de $S$. Suponha que $I \subseteq S$ é um $(S, R)$-bimódulo e que $M$ é um $S$-módulo à direita. Então

$$
M \otimes_{S} S / I \cong M / M I
$$

como R-módulos à direita.

Demonstração. [Cou95], Capítulo 12, Corolário 4.7.

Proposição B.3.7 (Extensão de Escalares). Seja $S$ um subanel de R.

1. Dado um bimódulo ${ }_{R} M_{S}$ e um módulo à esquerda ${ }_{S} N$, então o produto tensorial $M \otimes_{S} N$ é um $R$-módulo à esquerda, em que $r(x \otimes y)=(r x) \otimes y$, para todos $r \in R, x \in M e$ $y \in N$. Similarmente, dados $M_{S}$ e ${ }_{S} N_{R}$, o produto tensorial $M \otimes_{S} N$ é um R-módulo à direita, em que $(x \otimes y) r=x \otimes(y r)$, para todos $r \in R, x \in M$ e $y \in N$.

2. $O$ anel $R$ é um $(R, S)$-bimódulo e, se $M$ é um $S$-módulo à esquerda, então $R \otimes_{S} M$ é um $R$-módulo à esquerda, em que $r_{1}\left(r_{2} \otimes m\right)=\left(r_{1} r_{2}\right) \otimes m$, para todos $r_{1}, r_{2} \in R$ e $m$ $\in M$.

Demonstração. [Rot09], Capítulo 2, Proposição 2.51, pág. 76.

Teorema B.3.20. Sejam $R$ um anel, $M$ um $R$-módulo à direita e $\left(N_{i}\right)_{i \in I}$ uma família de $R$-módulos à esquerda. Existe um $Z(R)$-isomorfismo

$$
\tau: M \otimes_{R}\left(\bigoplus_{i \in I} N_{i}\right) \longrightarrow \bigoplus_{i \in I}\left(M \otimes_{R} N_{i}\right)
$$

$\operatorname{com} \tau: a \otimes\left(b_{i}\right) \mapsto\left(a \otimes b_{i}\right)$, em que $Z(R)$ é o centro do anel $R$. Em particular, se $R$ é comutativo, então $\tau$ é um R-isomorfismo.

Demonstração. [Rot09], Capítulo 2, Teorema 2.65, pág. 86.

Existe uma relação entre o funtor Hom e o funtor Tensor. Antes vamos enunciar dois teoremas. A ideia central é que uma função de duas variáveis $f: M \times N \rightarrow M^{\prime}$, pode ser vista como uma família de funções com um parâmetro: Se fixo $x \in M$, então definimos $f_{x}: N \rightarrow M^{\prime}$ por $y \mapsto f(x, y)$, para todos $y \in N$. Pela Proposição B.3.7, se $R, S$ são anéis e $M_{R}$ e ${ }_{R} N_{S}$ são módulos, então $M \otimes_{R} N$ é um $S$-módulo à direita, em que $(x \otimes y) s=x \otimes(y s)$, para todos $s \in S, x \in M$ e $y \in N$. Além disso, se $M_{S}^{\prime}$ é um módulo, então $\operatorname{Hom}_{S}\left(N, M^{\prime}\right)$ é um $R$-módulo à direita, em que $(f r)(y)=f(r y)$, para todos $f \in \operatorname{Hom}_{S}\left(N, M^{\prime}\right)$ e $r \in R$.

Então $\operatorname{Hom}_{R}\left(M, \operatorname{Hom}_{S}\left(N, M^{\prime}\right)\right)$ consiste de $R$-aplicações entre $R$-módulos à direita. Finalmente se $F \in \operatorname{Hom}_{R}\left(M, \operatorname{Hom}_{S}\left(N, M^{\prime}\right)\right)$, nós denotamos seu valor em $x \in M$ por $F_{x}$, então $F_{x}: N \rightarrow M^{\prime}$, definido por $F_{x}: y \mapsto F(x)(y)$, é uma família de funções de um parâmetro. 
Teorema B.3.21 (Isomorfismo de adjunção, primeira versão). Dados módulos $M_{R},{ }_{R} N_{S} e$ $M_{S}^{\prime}$, tais que $R, S$ são anéis, existe um isomorfismo natural

$$
\tau_{M, N, M^{\prime}}: \operatorname{Hom}_{S}\left(M \otimes_{R} N, M^{\prime}\right) \rightarrow \operatorname{Hom}_{R}\left(M, \operatorname{Hom}_{S}\left(N, M^{\prime}\right)\right)
$$

Se $f: M \otimes_{R} N \rightarrow M^{\prime}, x \in M, y \in N$ então $\tau_{M, N, M^{\prime}}: f \mapsto \tau(f)$, em que $\tau(f)_{x}: y \mapsto f(x \otimes y)$.

Demonstração. [Rot09], Capítulo 2, Teorema 2.75, pág. 92.

Teorema B.3.22 (Isomorfismo de adjunção, segunda versão). Dados módulos ${ }_{R} M,{ }_{S} N_{R} e$ ${ }_{S} M^{\prime}$, tais que $R, S$ são anéis, existe um isomorfismo natural

$$
\tau_{M, N, M^{\prime}}^{\prime}: \operatorname{Hom}_{S}\left(N \otimes_{R} M, M^{\prime}\right) \rightarrow \operatorname{Hom}_{R}\left(M, \operatorname{Hom}_{S}\left(N, M^{\prime}\right)\right)
$$

Se $f: N \otimes_{R} M \rightarrow M^{\prime}, x \in M, y \in N$ então $\tau_{M, N, M^{\prime}}^{\prime}: f \mapsto \tau^{\prime}(f)$, em que $\tau^{\prime}(f)_{x}: y \mapsto f(y \otimes x)$.

Demonstração. [Rot09], Capítulo 2, Teorema 2.76, pág. 93.

Existem duas versões de isomorfismos de adjunção, resultantes das duas maneiras nas quais $N$ pode ser um bimódulo $\left({ }_{R} N_{S}\right.$ ou $\left.{ }_{S} N_{R}\right)$.

Sejam $M_{R},{ }_{R} N_{S}$ e $M_{S}^{\prime}$ módulos, tais que $R, S$ são anéis. Como dito anteriormente $M \otimes_{R} N$ $\in \bmod -S$ e $\operatorname{Hom}_{S}\left(N, M^{\prime}\right) \in \bmod -R$. Então podemos considerar dois funtores:

$$
\operatorname{Hom}_{S}(N,-): \bmod -S \rightarrow \bmod -R
$$

$\mathrm{e}$

$$
-\otimes_{R} N: \bmod -R \rightarrow \bmod -S
$$

Pelo Teorema B.3.21, temos que $\operatorname{Hom}_{S}\left(M \otimes_{R} N, M^{\prime}\right) \cong \operatorname{Hom}_{R}\left(M, \operatorname{Hom}_{S}\left(N, M^{\prime}\right)\right)$. Portanto $\operatorname{Hom}_{S}(N,-)$ e $-\otimes_{R} N$ são um par de funtores adjuntos, ou seja, $-\otimes_{R} N$ é adjunto à esquerda de $\operatorname{Hom}_{S}(N,-)$ e $\operatorname{Hom}_{S}(N,-)$ é adjunto à direita de $-\otimes_{R} N$.

Analogamente, se considerarmos ${ }_{R} M,{ }_{S} N_{R}$ e ${ }_{S} M^{\prime}$ módulos, tais que $R, S$ são anéis, pelo Teorema B.3.22, concluiremos que $\operatorname{Hom}_{S}(N,-)$ e $N \otimes_{R}-$ são um par de funtores adjuntos,

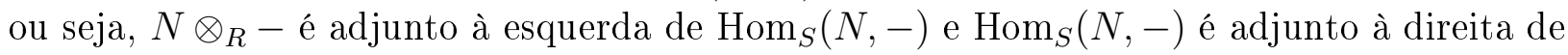
$N \otimes_{R}-$. Mostramos assim a relação entre o funtor Hom e o funtor Tensor.

\section{B.4 Referências}

Grande parte da Seção B.1 foi baseada em [Eti11] [Capítulo 7] e [Lan98] [Capítulo I]. Já a Seção B.2 foi baseada em [Rot03] [Capítulo 7] e [Lan98] [Capítulo VIII]. A Seção B.3 (Subseções: B.3.1, B.3.2, B.3.3, B.3.4, B.3.5 e B.3.6) foi baseada em [Nor73] [Capítulos 1 e 2] e em [Rot03] [Capítulos 7]. A Seção B.3 (Subseção: B.3.7) foi baseada em [Rot09] [Capítulo 2]. 


\section{Apêndice C}

\section{Localização}

\section{C.1 Localizações em anéis comutativos}

Como uma generalização da forma em que construímos o corpo de frações de um domínio de integridade, podemos construir a localização de um subconjunto multiplicativo de um anel como sendo o anel obtido invertendo formalmente os elementos deste subconjunto.

Vamos basear o Apêndice em [GJ89], Capítulos 5 e 9.

Suponhamos que $R$ seja um anel comutativo e $X$ um subconjunto de $R$. Queremos encontrar o "maior" anel no qual os elementos de $X$ tornam-se unidades.

Primeiramente, como todos os produtos dos elementos de $X$ devem necessariamente tornar-se unidades no nosso novo anel, podemos assumir que $X$ seja fechado sob multiplicação, e que $1=1_{R} \in X$. Então construiremos um novo anel $R X^{-1}$ (geralmente escrito $R_{X}$ na literatura comutativa, mas esta notação pode causar confusão no caso não comutativo) como o conjunto de frações $r / x$ em que $r \in R$ e $x \in X$. Deve existir uma relação de equivalência nestas frações, e deve ser tomada com cuidado pois $X$ pode conter divisores de zero, caso em que a aplicação $R \rightarrow R X^{-1}, r \mapsto r / 1$, não é injetiva. A relação de equivalência entre as frações é a seguinte: dizemos que $r / x$ e $r^{\prime} / x^{\prime}$ definem o mesmo elemento de $R X^{-1}$ se, e somente se, $\left(r x^{\prime}-r^{\prime} x\right) y=0$ para algum $y \in X$.

Em outras palavras, defina uma relação $\equiv \mathrm{em} R \times X$ como segue:

$$
(r, x) \equiv\left(r^{\prime}, x^{\prime}\right) \Longleftrightarrow\left(r x^{\prime}-r^{\prime} x\right) y=0 \text { para algum } y \in X
$$

Claramente, esta relação é reflexiva e simétrica. Para ver que é transitiva, suponha que $(r, x) \equiv\left(r^{\prime}, x^{\prime}\right)$ e $\left(r^{\prime}, x^{\prime}\right) \equiv(s, y)$. Então existem $v, w \in X$ tais que $\left(r x^{\prime}-r^{\prime} x\right) v=0$ e $\left(r^{\prime} y-s x^{\prime}\right) w=0$. Eliminando $r^{\prime}$ destas duas equações temos $(r y-s x) x^{\prime} v w=0$. Como $X$ é fechado sob multiplicação temos que $x^{\prime} v w \in X$, logo $(r, x) \equiv(s, y)$. Portanto, $\equiv$ é uma relação de equivalência.

Denotamos por $r / x$ a classe de equivalência de $(r, x)$ e seja $R X^{-1}=(R \times X) / \equiv \mathrm{o}$ conjunto das classes de equivalências. A estrutura de anel em $R X^{-1}$ é definida da seguinte maneira usual:

$$
\frac{r_{1}}{x_{1}}+\frac{r_{2}}{x_{2}}=\frac{r_{1} x_{2}+r_{2} x_{1}}{x_{1} x_{2}} \text { e } \frac{r_{1}}{x_{1}} \cdot \frac{r_{2}}{x_{2}}=\frac{r_{1} r_{2}}{x_{1} x_{2}}
$$

Estas operações estão bem definidas, isto é, não dependem dos representantes de classe utilizados. Com estas operações, $R X^{-1}$ é um anel comutativo (com elementos neutro 0/1 e identidade 1/1) que chamamos de localização de $R$ com respeito a $X$. Na verdade é um abuso de linguagem, pois associado à $R X^{-1}$ temos um homomorfismo de anéis $\phi: R \rightarrow R X^{-1}$ dado 
por $r \mapsto r / 1$ chamado de mapa de localização e o par $\left(R X^{-1}, \phi\right)$ é a localização de $R$ com respeito a $X$. Quando $R$ é um domínio de integridade e $X=R \backslash\{0\}$ então $R X^{-1}$ é o corpo de frações de $R$ e neste caso o mapa de localização é a inclusão $R \subseteq R X^{-1}$. Entretanto, para anéis gerais, o mapa de localização nem sempre é injetivo.

$\mathrm{O}$ anel $R X^{-1}$ juntamente com seu mapa de localização tem as seguintes propriedades:

(i) Para cada $x \in X$, o elemento $\phi(x)$ é uma unidade de $R X^{-1}$;

(ii) O kernel de $\phi$ é $\{r \in R \mid r x=0$ para algum $x \in X\}$;

(iii) Se $\psi: R \rightarrow T$ é um homomorfismo de anéis qualquer tal que $\psi(x)$ é uma unidade de $T$ para todo $x \in X$ e $T$ seja comutativo, então $\psi$ se fatora unicamente através de $\phi$, isto é, existe um único homomorfismo de anéis $\eta: R X^{-1} \rightarrow T$ tal que $\psi=\eta \phi$.

O item (iii) descrito acima é conhecido como a Propriedade Universal da Localização (para o caso comutativo). Vamos mostrar a unicidade. Se $\eta$ satisfaz a condição então $\eta(r / 1)=$ $\eta \phi(r)=\psi(r)$, para todo $r \in R$, logo se $x \in X, \eta(1 / x)=\eta\left((x / 1)^{-1}\right)=\psi(x)^{-1}$. Assim $\eta(r / x)=\eta(r / 1) \eta(1 / x)=\psi(r) \psi(x)^{-1}, \operatorname{logo} \eta$ é univocamente determinado por $\psi$. Agora vejamos a existência. Seja $\eta(r / x)=\psi(r) \psi(x)^{-1}$. Então $\eta$ será claramente um homomorfismo de anéis desde que esteja bem definido. Suponha que $r / x=r^{\prime} / x^{\prime}$, então existe $t \in X$ tal que $\left(r x^{\prime}-r^{\prime} x\right) t=0$. Aplicando $\psi$ temos $\left(\psi(r) \psi\left(x^{\prime}\right)-\psi\left(r^{\prime}\right) \psi(x)\right) \psi(t)=0$. Agora $\psi(t), \psi(x)$ e $\psi\left(x^{\prime}\right)$ são unidades em $T$, então $\psi(r) \psi(x)^{-1}=\psi\left(r^{\prime}\right) \psi\left(x^{\prime}\right)^{-1}, \operatorname{logo} \eta(r / x)=\eta\left(r^{\prime} / x^{\prime}\right)$.

Estender essa simples ideia para o caso não comutativo não é tão simples. A questão geral foi considerada pela primeira vez por Ore, que investigou se um domínio não comutativo $R$ pode ser "mergulhado" em um anel de divisão $D$, tal que todo elemento de $D$ seja da forma $r x^{-1}$ para alguns $r, x \in R \operatorname{com} x \neq 0$.

\section{C.2 Localizações em anéis não comutativos}

Nesta seção $R$ denota um anel (ou domínio) não comutativo.

Definição C.2.1. Um domínio de Ore à direita é qualquer domínio $R$ no qual quaisquer dois elementos não nulos possuem um múltiplo à direita comum, isto é, para cada $x, y \in R$ não nulos existem $r, s \in R$ tais que $x r=y s \neq 0$. Definimos domínio de Ore à esquerda de maneira simétrica.

Por exemplo, todo domínio comutativo (ou seja, domínio de integridade) é de Ore à direita.

Devemos tomar cuidado para distinguir as frações com denominadores à direita, tais como $a b^{-1}$, das frações com denominadores à esquerda, tais como $c^{-1} d$.

Lema C.2.1. Para um dominio $R$, as seguintes condições são equivalentes:

1. $R$ é um domínio de Ore à direita.

2. $R_{R}$ é uniforme.

3. $R_{R}$ tem posto finito.

Demonstração. [GJ89], Capítulo 5, Lema 5.15, pág. 94.

Definição C.2.2. Um módulo $M$ tem posto finito se seu envelope injetivo é uma soma direta finita de submódulos indecomponíveis. 
Definição C.2.3. Um módulo uniforme é um módulo $M$ não nulo tal que a intersecção de quaisquer dois submódulos não nulos de $M$ é não nula, ou equivalentemente, tal que todo submódulo não nulo de $M$ é essencial em $M$.

Corolário C.2.1. Todo domínio Noetheriano à direita é domínio de Ore à direita.

Demonstração. [GJ89], Capítulo 5, Corolário 5.16, pág. 94.

Definição C.2.4. Seja $R$ um anel. Uma ordem à direita em $R$ é qualquer subanel $S \subseteq R$ tal que:

1. Todo elemento regular de $S$ é invertivel em $R$;

2. Todo elemento de $R$ tem a forma $a b^{-1}$ para algum $a \in S$ e algum regular $b \in S$.

Uma ordem à esquerda é definida analogamente, usando frações da forma $b^{-1} a$. No caso em que ambos anéis são comutativos, os adjetivos "direita" e "esquerda" não são necessários.

Teorema C.2.1 (Ore). Um anel $R$ é uma ordem à direita em um anel de divisão se, e somente se, $R$ é um dominio de Ore à direita.

Demonstração. [GJ89], Capítulo 5, Teorema 5.17, pág. 94.

O Teorema C.2.1 nos mostra que a investigação de Ore citada anteriormente é falsa em geral, mas Ore conseguiu encontrar uma condição necessária e suficiente para que seja verdade: um anel de divisão $D$ como descrito anteriormente existe se, e somente se, quaisquer dois elementos não nulos de $R$ tem um múltiplo à direita comum não nulo. A necessidade desta condição do múltiplo comum é imediata da forma especial dos elementos de $D$, pois se $r, s$ são elementos não nulos de $R$, então $D$ contém o elemento $s^{-1} r$, que deve ser da forma $x y^{-1}$ para alguns $x, y \in R$ não nulos, e a equação $s^{-1} r=x y^{-1}$ em $D$ resulta na equação $r y=s x$ em $R$.

Esta condição do múltiplo à direita comum não nulo é conhecida como condição de Ore à direita (embora devemos usar o termo em um contexto mais geral).

Observamos pelo Corolário C.2.1 que um domínio Noetheriano à direita sempre satisfaz a condição de Ore. Este fato não foi notado por muitos anos, provavelmente porque a importância da condição noetheriana não era clara.

Lembremos que a álgebra de Weyl é um domínio não comutativo e como podemos notar, o procedimento para tomar localizações destes, não é direto como no caso comutativo. Existem algumas condições necessárias e suficientes para que a localização exista.

Definição C.2.5. Seja $R$ um anel e $X$ um conjunto multiplicativo em $R$, ou seja, $1 \in X$ e $X$ é fechado sob multiplicação. Uma localização de Ore à direita para $R$ com respeito a $X$ é um homomorfismo de anéis $\phi: R \rightarrow S$ tal que:

1. $\phi(x)$ é uma unidade de $S$ para todo $x \in X$.

2. Todo elemento de $S$ tem a forma $\phi(r) \phi(x)^{-1}$ para alguns $r \in R, x \in X$.

3. $\operatorname{ker}(\phi)=\{r \in R \mid r x=0$ para algum $x \in X\}$.

$A$ localização de Ore à esquerda pode ser introduzida de maneira simétrica. 
A localização de Ore à direita (à esquerda) também pode ser chamada de anel de frações

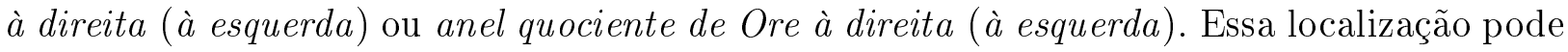
ser vista como um par $(S, \phi)$ que satisfaz as propriedades citadas na definição, mas por abuso de notação, usualmente nos referimos à $S$ como a localização de Ore à direita (à esquerda) e escrevemos os elementos de $S$ na forma $r x^{-1}$ para alguns $r \in R, x \in X$.

Caso uma localização à direita (ou à esquerda) exista, veremos adiante que ela satisfaz a Propriedade Universal desejada (semelhante ao caso comutativo).

Por satisfazer essa Propriedade Universal, claramente se a localização à direita (à esquerda) existir, ela é única a menos de um isomorfismo que é a identidade em $R$.

Lema C.2.2. Seja $X$ um conjunto multiplicativo em um anel $R$, e assuma que existe a localização de Ore à direita para $R$ com respeito a $X$. Então:

1. $r X \cap x R \neq \varnothing$ para todos $r \in R, x \in X$.

2. Se $r \in R$ e $x \in X$ tal que $x r=0$, então existe $x^{\prime} \in X$ tal que $r x^{\prime}=0$.

Demonstração. [GJ89], Capítulo 9, Lema 9.1, pág. 144.

Definição C.2.6. Seja $X$ um conjunto multiplicativo em um anel R. Então $X$ satisfaz a condição de Ore à direita se, e somente se, $X$ satisfaz a condição 1 do Lema C.2.2, enquanto que $X$ é reversível à direita se, e somente se, $X$ satisfaz a condição 2.

Definição C.2.7. Um conjunto de Ore à direita é qualquer conjunto multiplicativo satisfazendo a condição de Ore à direita. Um conjunto de denominadores à direita é qualquer conjunto de Ore à direita reversivel à direita, ou seja, que satisfaz as condições do Lema C.2.2.

Posteriormente veremos que, a localização de Ore à direita existe precisamente para conjuntos de denominadores à direita.

Lema C.2.3. Seja $X$ um conjunto de Ore à direita em um anel $R$.

1. Dados quaisquer $x_{1}, \ldots, x_{n} \in X$, o conjunto $x_{1} R \cap \ldots \cap x_{n} R \cap X$ é não vazio.

2. Agora suponhamos que $\phi: R \rightarrow T$ é um homomorfismo de anéis tal que $\phi(x)$ é uma unidade de $T$ para todo $x \in X$. Dados quaisquer $r_{1}, \ldots, r_{n} \in R$ e $x_{1}, \ldots, x_{n} \in X$, existem $s_{1}, \ldots, s_{n} \in R$ e $y \in X$ tais que $r_{i} x_{i}^{-1}=s_{i} y^{-1}$ (isto é, as "frações" $r_{i} x_{i}^{-1}$ tem um "denominador comum" $y$ ).

3. $O$ conjunto $S=\left\{r x^{-1} \mid r \in R\right.$ e $\left.x \in X\right\}$ é um subanel de $T$.

Demonstração. [GJ89], Capítulo 9, Lema 9.2, pág. 145.

Lema C.2.4. Seja $X$ um conjunto de Ore à direita em um anel $R$. Se $M$ é qualquer $R$ módulo à direita, o conjunto $\{a \in M \mid a x=0$ para algum $x \in X\}$ é um submódulo de $M$.

Demonstração. [GJ89], Capítulo 9, Lema 9.3, pág. 145.

Definição C.2.8. Seja $X$ um conjunto de Ore à direita em um anel $R$. Para qualquer $R$ módulo à direita $M$, o submódulo $t_{X}(M)=\{a \in M \mid a x=0$ para algum $x \in X\}$ é chamado de $X$-submódulo de torção de $M$. O módulo $M$ é $X$-torção se, e somente se, $t_{X}(M)=M$ e o módulo $M$ é $X$-livre de torção se, e somente se, $t_{X}(M)=\{0\}$. 
Notemos que $t_{X}(R)$ é um ideal de $R$, e se existe a localização de Ore à direita $(S, \phi)$ para $R$ com respeito a $X$, então $\operatorname{ker}(\phi)=t_{X}(R)$.

Agora podemos enunciar a Propriedade Universal:

Proposição C.2.1. Seja $X$ um conjunto de denominadores à direita em um anel $R$, $e$ suponha que a localização de Ore à direita $\phi: R \rightarrow S$ para $R$ com respeito a $X$ existe. Se $\psi: R \rightarrow T$ é um homomorfismo de anéis qualquer, tal que $\psi(x)$ é uma unidade em $T$ para todo $x \in X$, então existe um único homomorfismo de anéis $\eta: S \rightarrow T$ tal que $\psi=\eta \phi$.

Demonstração. [GJ89], Capítulo 9, Proposição 9.4, pág. 146.

Corolário C.2.2. Seja $X$ um conjunto de denominadores à direita em um anel $R$, e suponha que $\phi_{1}: R \rightarrow S_{1}$ e $\phi_{2}: R \rightarrow S_{2}$ são duas localizações de Ore à direita para $R$ com respeito a $X$. Então existe um (único) isomorfismo de anéis $\eta: S_{1} \rightarrow S_{2}$ tal que $\eta \phi_{1}=\phi_{2}$.

Demonstração. [GJ89], Capítulo 9, Corolário 9.5, pág. 146.

Observamos que o que foi feito acima, pode ser feito para a localização de Ore à esquerda, simetricamente. Ainda não estabelecemos que a localização de Ore à direita (à esquerda) existe. Vejamos então as condições para a existência.

Teorema C.2.2. Seja X um conjunto multiplicativo em um anel R. Então existe a localização de Ore à direita para $R$ com respeito a $X$ se, e somente se, $X$ é um conjunto de denominadores à direita.

Demonstração. [GJ89], Capítulo 9, Teorema 9.7, pág. 148. Observamos que a condição necessária é dada pelo Lema C.2.2.

O Teorema acima nos dá a condição suficiente para a existência da localização.

Dado um conjunto de denominadores à direita $X$ em um anel $R$, podemos ver explicitamente a localização de Ore à direita para $R$ com respeito a $X$. Defina uma relação de equivalência $\equiv$ em $R \times X$, em que $(a, x) \equiv(b, y)$ se, e somente se, existem $c \in R, d$ $\in X$ com $a c=b d$ e $x c=y d \in X$. Seja $((a, x))$ a classe de equivalência de $(a, x)$. Assim $S=(R \times X) / \equiv$ é o conjunto de todas as classes de equivalência. Vamos definir as operações de soma e produto em $S$. Dados $((a, x)),((b, y)) \in S$, e tomando $c \in R, d \in X$ com $x c=y d$, define-se $((a, x))+((b, y))=((a c+b d, x c))$. Escolhendo $c \in R, z \in X$ com $b z=x c$, define-se $((a, x)) \cdot((b, y))=((a c, y z))$. Tais soma e produto estão bem definidos. Portanto, $S$ é um anel, e a aplicação $r \mapsto((r, 1))$ define um isomorfismo de $R$ em $S$, identificando $R$ como subanel de $S$. Portanto $S$ é uma localização de Ore à direita para $R$ com respeito a $X$.

Definição C.2.9. Dado um conjunto de denominadores à direita $X$ em um anel $R$, sabemos que existe a localização de Ore à direita $\phi: R \rightarrow S$. Pela unicidade, dada pelo Corolário C.2.2, podemos denotar $S$ por $R X^{-1}$, e nos referir à $\phi$ como a inclusão natural de $R$ para $R X^{-1}$. No caso da localização de Ore à esquerda com respeito ao conjunto de denominadores $Y$, denotaremos a localização por $Y^{-1} R$.

Proposição C.2.2. Se $X$ é um conjunto de denominadores à direita e à esquerda em um anel $R$, então qualquer localização de Ore à direita (à esquerda) para $R$ com respeito a $X$ é também uma localização de Ore à esquerda (à direita) para $R$ com respeito a $X$. Em outras palavras $X^{-1} R=R X^{-1}$.

Demonstração. [GJ89], Capítulo 9, Proposição 9.8, pág. 149. 


\section{C.3 Localizações em módulos}

Nesta seção $R$ denota um anel (ou domínio) não comutativo.

Tendo construído a localização de Ore à direita $R X^{-1}$ de um dado conjunto de denominadores à direita $X$ em um anel $R$, vamos construir uma localização $M X^{-1}$ para cada $R$-módulo à direita $M$. Claramente os elementos de $M X^{-1}$ serão "frações" com numerador pertencente à $M$ e denominador à $X$. O kernel da aplicação de $M$ para $M X^{-1}$ será $t_{X}(M)$, e a aplicação $M \rightarrow M X^{-1}$ terá uma Propriedade Universal com respeito a homomorfismos de $R X^{-1}$-módulos.

Definição C.3.1. Sejam $X$ um conjunto de denominadores à direita em um anel $R$ e $M$ um $R$-módulo à direita. Uma localização do módulo $M$ com respeito a $X$ (ou módulo de frações para $M$ com respeito a $X$ ) consiste de um $R X^{-1}$-módulo à direita $N$ munido com um $R$-homomorfismo $f: M \rightarrow N$ tal que:

1. Todo elemento de $N$ tem a forma $f(a) x^{-1}$ para alguns $a \in M, x \in X$.

2. $\operatorname{ker}(f)=t_{X}(M)$.

Com abuso de notação, geralmente nos referimos à $N$ como a localização do módulo $M$.

Proposição C.3.1. Sejam X um conjunto de denominadores à direita em um anel $R$ e $M$ um $R$-módulo à direita, e suponha que exista a localização $f: M \rightarrow N$ para $M$ com respeito a $X$. Se $P$ é um $R X^{-1}$-módulo à direita e $g: M \rightarrow P$ é um $R$-homomorfismo, existe um único homomorfismo de $R X^{-1}$-módulos $h: N \rightarrow P$ tal que $g=h f$.

Demonstração. [GJ89], Capítulo 9, Proposição 9.10, pág. 151.

Corolário C.3.1. Sejam $X$ um conjunto de denominadores à direita em um anel $R$ e $M$ um $R$-módulo à direita, e suponha que $f_{1}: M \rightarrow N_{1}$ e $f_{2}: M \rightarrow N_{2}$ são localizações para $M$ com respeito a $X$. Então existe um (único) isomorfismo de $R X^{-1}$-módulos $h: N_{1} \rightarrow N_{2}$ tal que $h f_{1}=f_{2}$.

Demonstração. [GJ89], Capítulo 9, Corolário 9.11, pág. 151.

Definição C.3.2. Seja $X$ um conjunto multiplicativo em um anel $R$. Dizemos que um $R$ módulo à direita $M$ é $X$-divisível se, e somente se, $M x=M$ para todo $x \in X$.

Proposição C.3.2. Seja X um conjunto de denominadores à direita em um anel $R$.

1. Todo $R X^{-1}$-módulo à direita ou esquerda é X-livre de torção e X-divisível como $R$ módulo.

2. Se $M$ é um R-módulo à direita X-livre de torção e X-divisivel, existe uma única estrutura de $R X^{-1}$-módulo à direita em $M$ compativel com sua estrutura de $R$-módulo à direita.

Demonstração. [GJ89], Capítulo 9, Proposição 9.12, pág. 152.

Vamos enunciar a existência da localização de um módulo:

Teorema C.3.1. Se $X$ é um conjunto de denominadores à direita em um anel $R$, então existe uma localização para qualquer $R$-módulo à direita $M$ com respeito a $X$.

Demonstração. [GJ89], Capítulo 9, Teorema 9.13, pág. 152. 
Definição C.3.3. Dados um conjunto de denominadores à direita $X$ em um anel $R$ e um $R$-módulo à direita $M$, sabemos que existe a localização $f: M \rightarrow N$ para $M$ com respeito a X. Por conta da unicidade dada pelo Corolário C.3.1, podemos denotar $N$ por $M X^{-1} e$ $f$ será a inclusão natural de $M$ para $M X^{-1}$. Escrevemos os elementos de $M X^{-1}$ na forma $a x^{-1}$, e elementos de $f(M)$ na forma $a 1^{-1}$.

Suponhamos que $P$ é outro $R$-módulo à direita, e seja $g: M \rightarrow P$. Como visto na Proposição C.3.1, existe um único homomorfismo de $R X^{-1}$-módulos à direita $h: M X^{-1} \rightarrow$ $P X^{-1}$ tal que $h\left(a x^{-1}\right)=g(a) x^{-1}$ para todos $a \in M, x \in X$. Nos referimos a $h$ como $a$ aplicação induzida por $g$, e se a notação para $h$ é necessária, usamos $g X^{-1}$.

A seguinte proposição mostra que qualquer localização $M X^{-1}$ sobre a localização de Ore à direita $R X^{-1}$ é naturalmente isomorfa a $M \otimes_{R} R X^{-1}$. Então o produto tensorial nos fornece uma abordagem alternativa para a localização de $R$-módulos.

Proposição C.3.3. Sejam $X$ um conjunto de denominadores à direita em um anel $R$ e $M$ um R-módulo à direita. Então a "aplicação multiplicação" $M \times R X^{-1} \rightarrow M X^{-1}$ dada por $(a, s) \mapsto\left(a 1^{-1}\right) s$ induz um isomorfismo de $R X^{-1}$-módulos entre $M \otimes_{R} R X^{-1}$ e $M X^{-1}$.

Demonstração. [GJ89], Capítulo 9, Proposição 9.14, pág. 153.

\section{C.4 Referências}

Todas as seções foram baseadas em [GJ89][Capítulos 5 e 9]. 

APÊNDICE C 


\section{Referências Bibliográficas}

[Abr83] G. Abrams. Morita equivalence for rings with local units. Comm. Algebra, 11(8):801-837, 1983. 91

[ASS06] I. Assem, D. Simson e A. Skowrónski. Elements of the Representation Theory of Associative Algebras - volume 1. London Math. Soc. - Students Texts 65. Cambridge University Press, 2 edição, 2006. 59, 89

[Bav92a] V. Bavula. Generalized Weyl algebras and their representations. Algebra i Analiz, 4(1):75-97, 1992. English transl.: St Petersburg Math. J. 4(1):71-92, 1993. x, 25, $27,65,73,77,84$

[Bav92b] V. Bavula. The simple $D[X, Y ; \sigma, a]$-modules. Ukrainian Math. J., 44:1628-1644, 1992. $65,73,77$

[BB00] V. Bavula e V. Bekkert. Indecomposable representations of generalized Weyl algebras. Comm. Algebra, 28:5067-5100, 2000. ix, xi, 25, 27, 46, 65, 67, 73, 77, 78, 80

[BBF04] V. Bekkert, G. Benkart e V. Futorny. Weight modules for Weyl algebras in KacMoody Lie algebras and related topics. Contemp. Math.;Amer. Math. Soc., 343:1742, 2004. i, iii, ix, 25, 27, 65, 77, 83, 91

[BBL97] G. M. Benkart, D. J. Britten e F. W. Lemire. Modules with bounded weight multiplicities for simple Lie algebras. Math. Zeitschrift, 225:333-353, 1997. 65

[Bea99] J. A. Beachy. Introductory Lectures on Rings and Modules. London Math. Soc. Students Texts 47. Cambridge University Press, 1 edição, 1999. 52, 53, 63

[BF85] J. Backelin e R. Fröberg. Koszul algebras, Veronese subrings and rings with linear resolutions. Rev. Roumaine Math. Pures Appl., 30(2):85-97, 1985. 90

[Bjö79] J.-E. Björk. Rings of differential operators. North Holland Mathematical Library, Amsterdam, 1979. 12

[Bla77] B. Blackadar. Infinite tensor products of $C^{*}$-algebras. Pac. J. Math., 77:313-334, 1977. 89

[Blo81] R. E. Block. The irreducible representations of the Lie algebra $\mathfrak{s l}(2)$ and of the Weyl algebra. Adv. Math., 39:69-110, 1981. 65, 77

[Bro82] K. S. Brown. Cohomology of groups. Springer-Verlag, 1 edição, 1982. 48, 63

[BvO97] V. Bavula e F. van Oystaeyen. The simple modules of certain generalized crossed products. J. Algebra, 194:521-566, 1997. 65, 66, 73, 77 
[BvO00] V. Bavula e F. van Oystaeyen. Simple holonomic modules over the second Weyl algebra $A_{2}$. Adv. Math, 150:80-116, 2000. 65

[Cou95] S. C. Coutinho. A Primer of Algebraic D-modules. London Math. Soc. - Student Texts 33. Cambridge University Press, 1 edição, 1995. 2, 12, 27, 29, 30, 31, 37, 43, 63,135

[DGO96] Yu. A. Drozd, B. L. Guzner e S. A. Ovsienko. Weight modules over generalized Weyl algebras. J. of Algebra, 184:491-504, 1996. xi, 65, 67, 69, 73, 77, 78, 80

[DOF94] Yu. A. Drozd, S. A. Ovsienko e V. M. Futorny. Harish-Chandra subalgebras and Gelfand-Zetlin modules, in "Finite Dimensional Algebras and Related Topics". Kluwer Academic, 1994. 74

[Dro80] Yu. A. Drozd. Tame and wild matrix problem. in Representation Theory II (Proc. Second Internat. Conf. Carleton Univ. Ottawa Ontario 1979). Springer Lec. Notes in Math., 832:242-258, 1980. 77

[Eti11] P. Etingof. ...[et al.]Introduction to Representation Theory. Amer. Math. Soc. Student Mathematical Library vol.59, 2011. 97, 115, 121, 124, 136

[FGM14] V. Futorny, D. Grantcharov e V. Mazorchuk. Weight modules over infinite dimensional Weyl algebras. Proceedings of the Amer. Math. Soc., 142(9):3049-3057, 2014. i, iii, ix, x, xi, 17, 27, 44, 46, 57, 62, 63, 83, 84, 87, 88, 89, 91

[GJ89] K. R. Goodearl e R. B. Warfield Jr. An introduction to Noncommutative Noetherian Rings. London Math. Soc. - Student Texts 16. Cambridge University Press, 1 edição, 1989. 84, 86, 137, 138, 139, 140, 141, 142, 143

[GP68] I. M. Gelfand e V. A. Ponomarev. Indecomposable representations of the Lorenz group. Uspekhi Mat. Nauk, 23(2):3-40, 1968. English transl.: Russian Math. Surveys 23:1-58, 1968. 65

[GR97] P. Gabriel e A. V. Roiter. Representations of Finite-Dimensional Algebras. Springer-Verlag, Berlin, 1997. 67

[GS06] D. Grantcharov e V. Serganova. Category of $\mathfrak{s p}(2 n)$-modules with bounded weight multiplicities. Mosc. Math. J., 6(1):119-134, 2006. ix, 88

[Hum13] J. E. Humphreys. Introduction to Lie Algebras and Representation Theory. Springer-Verlag, 2013. 97, 100, 102, 103, 104, 105, 106, 107, 108, 109, 111, 112, 113

[Jac79] N. Jacobson. Lie Algebras. Dover Publications, Inc, New York, 2 edição, 1979. 99

[Jac80] N. Jacobson. Basic Algebra - Volume II. W. H. Freeman and Company, 3 edição, 1980. 22

[Lam98] T. Y. Lam. Lectures on Modules and Rings. Springer-Verlag, 2 edição, 1998. 60, 63

[Lam01] T. Y. Lam. A First Course in Noncommutative Rings. Springer-Verlag, 2 edição, 2001. 52, 53, 63, 93, 113 
[Lan98] S. Mac Lane. Categories for the Working Mathematician. Springer-Verlag, New York, Heidelberg, Berlin, 3 edição, 1998. 115, 123, 125, 136

[Mar10] L. A. B. San Martin. Algebras de Lie. Editora da Unicamp, 2 edição, 2010. 97, 102,103

[Mat00] O. Mathieu. Classification of irreducible weight modules. Ann. Inst.Fourier, 50(2):537-592, 2000. 88

[MZ11] V. Mazorchuk e K. Zhao. Characterization of simple highest weight modules. 2011. preprint arXiv: 1105.1123, para aparecer em Canada Math. Bull. 84

[Nor73] D. G. Northcott. A first course of homological algebra. Cambridge University Press, 1973. 115, 126, 127, 129, 130, 131, 132, 136

[Rot03] J. J. Rotman. Advanced modern algebra. Amer. Math. Soc., 2003. 93, 94, 96, 113, $115,118,119,122,123,131,132,136$

[Rot09] J. J. Rotman. An Introduction to Homological Algebra. Springer-Verlag, 2 edição, 2009. 58, 115, 130, 133, 134, 135, 136

[Sam90] H. Samelson. Notes on Lie Algebras. Springer-Verlag, 1990. 97, 109

[Smi85] S. P. Smith. Differential operators on $\mathbb{A}^{1}$ and $\mathbb{P}^{1}$ in char $\mathrm{p}>0$. Em Séminaire d'Algèbre Paul Dubreil et Marie-Pau Malliavin, Lecture Notes in Mathematics 1220, páginas 157-177. Springer-Verlag, 2 edição, 1985. 17

[Sta78] J. T. Stafford. Module structure of Weyl algebras. J. London Math. Soc., 18:429442, 1978. 12

[SZ91] P. Samuel e O. Zariski. Commutative Algebra - Volume I. Springer-Verlag, 1 edição, 1991. 18, 22 